\title{
VARIAÇÃO TEMPORAL DO BALANÇO HÍDRICO E DO CLIMA, DE ACORDO COM OS CRITÉRIOS DE KÖPPEN(1918) E THORNTHWAITE (1948), NA REGIÃO DE PIRACICABA - SP
}

\author{
ZILDA DE FÁTIMA MARIANO \\ Geógrafa
}

Orientador: Prof. Dr. VALTER BARBIERI

\begin{abstract}
Dissertação apresentada à Escola Superior de Agricultura
"Luiz de Queiroz", Universidade de São Paulo, para obtenção do título de Mestre em Agronomia, Área de Concentração: Agrometeorologia
\end{abstract}

P I R A C I C A B A

Estado de São Paulo - Brasil

Novembro-1998 


\section{ERRATA}

Página $12,1^{\circ}$ Parágrafo: Onde se lê potencial, leia-se evapotranspiração potencial.

Página $12,3^{\circ}$ Parágrafo: Onde se lê figura 1, leia-se figura 2.

Página 16, Figura 3: Onde se lê $\mathrm{B}_{4}$, leia-se $\mathrm{B}_{4}$.

Página $17,2^{\circ}$ Parágrafo: Onde se lê figura 2, leia-se figura 3.

Página 20, Tabela 1: Onde se lê $9 \mathrm{~mm}$, leia-se 9.

Página 20, Tabela 1: Onde se lê $10 \mathrm{~mm}$, leia-se 10.

Página $21,1^{\circ}$ Parágrafo: Onde se lê $9 \mathrm{~mm}$, leia-se 9.

Página $21,1^{\circ}$ Parágrafo: Onde se lê $10 \mathrm{~mm}$, leia-se 10.

Página 22, Tabela 2: Onde se lê $9 \mathrm{~mm}$, leia-se 9.

Página 22, Tabela 2: Onde se lê $10 \mathrm{~mm}$, leia-se 10.

Página 27, $1^{\circ}$ Parágrafo: Onde se lê mensal mensal, leia-se mensal normal.

Página 28, Tabela 6: Onde se lê BHND, leia-se BHDN.

Página 67, Último parágrafo: Onde se lê apresentado, leia-se apresentando. 
Dados Internacionais de Catalogação na Publicação (CIP) DIVISÃo DE BIBLIOTECA E DOCUMENTAÇÃO - Campus "Luiz de Queiroz"/USP

\author{
Mariano, Zilda de Fátima \\ Variação temporal do balanço hídrico e do clima, de acordo com os critérios de \\ Kōppen (1918) e Thornthwaite (1948), na regiāo de Piracicaba - SP / Zilda de \\ Fátima Mariano. - - Piracicaba, 1998. \\ 90 p. : il. \\ Dissertação (mestrado) - Escola Superior de Agricultura Luiz de Queiroz, 1998. \\ Bibliografia. \\ 1. Balanço hidrico 2. Classificação 3. Climatologia agricola 4. Chuva I. Título
}


Aos meus pais, Quirino e Vergínia;

irmãos, José Carlos, Rosa, Irene,

Leonice, Cláudio e Adriana; sobrinhos (as),

cunhados e à memória de minha irmã Maria Helena, que com muita dedicação sempre manifestaram apoio, incentivo e entenderam minha ausência.

\section{A MINHA IMENSA GRATIDÃo}

A DEUS e aos guias espirituais, pela fé, perseverança, saúde e por todas as graças concedidas durante o curso e na minha vida.

OFEREÇO

A todos (as) amigos (as) do Norte, Nordeste, Sul, Sudeste e Centro-Oeste do Brasil e os demais países da América do Sul, que ao meu lado repartiram todos os sentimentos de alegria, angústia, decepções, vitórias e derrotas que deram sabor de vitória. 


\section{AGRADECIMENTOS}

Ao Prof. Dr. Valter Barbieri, pela orientação, apoio e confiança depositada em meu trabalho;

Aos Professores do Departamento de Física e Meteorologia: Antonio Roberto Pereira, Nilson Augusto Villa Nova, Luiz Roberto Angelocci, Sérgio Moraes, Klaus Reichardt, Paulo Libardi, pela disposição nos ensinamentos para minha formação;

Aos mestres Octávio Freire e José Tadeu Garcia Tommaselli, que acreditaram e incentivaram minha vinda para ESALQ;

Ao Conselho Nacional de Pesquisa (CNPq) pelo apoio financeiro;

Aos funcionários do Departamento de Física e Meteorologia: Ana Maria M. Michelon, Márcia, Robson Tuon, Valderlino, Edvaldo, Francisco e Fernando;

À Professora Maria Izalina Ferreira Alves, pela dedicação na parte estatística.

À CAVE pela moradia cedida e o pelo apoio;

Aos funcionários da Biblioteca, pela enorme paciência;

Ao serviço médico da ESALQ, especialmente à Marta, pela dedicação e empenho nos meus pedidos;

Aos meus amigos de moradia: Jorge, Sebastião, Elton, Heron, Jailson (Rato), Antônio, Norberto, Quelmo, Marcelo, Walter, Rafael e, em especial, às minhas amigas e irmãs: Ana Cláudia Lira, Cristina Lacerda, Betânia Lúcia, Sylvia Elaine Farias e Jeane Portela, pela amizade, companheirismo e convívio;

Aos meus amigos do curso de Pós-Graduação: José Gabriel da Silva, Juan Delgado Rojas, José Ricardo Pezzopane, Adriana Vieira Camargo e Angélica Piccinni, pela amizade, aprendizagem e harmonioso convívio;

Em especial, às minhas grandes irmãs e amigas, Lucieta Guerreiro Martorano, Rosa Maria Nascimento dos Santos e Rosana Alves Ribas Moragas, pela grande amizade, convívio, dedicação e aprendizagem;

Ao Tom, minha querida mãe adotiva Joelita (Nita), seus filhos e netos pela expressão de amor verdadeiro.

Ao Marcos Barros de Souza, pelo incentivo, dedicação, paciência e força na minha vida; 
Ao Saint-Clair Trindade Jr, Genyton Rocha e Jadson Rabelo Porto, pela maravilhosa apresentação da região Norte e pela confiança e apoio em mim depositados;

A todos os pós-graduandos do Departamento de Irrigação e Drenagem, em especial aos baianos Francisco Adriano Pereira e Luiz Campeche.

Ao meu amigo, Professor Francisco Casimiro Filho, da UNIOESTE, Campus de Toledo, minha enorme gratidão;

Aos meus recentes amigos, professores da UNIOESTE, Campus de Marechal Cândido Rondon: Ivonete Pereira e Evaldo Mendes da Silva, que estão fazendo meus dias serem agradáveis e felizes em Marechal Cândido Rondon;

Aos funcionários da Seção de Informática da UNIOESTE, Campus de Marechal Cândido Rondon, Paulo Kozen e Emilson Kaiser, pela paciência, e pelos acertos finais da dissertação;

Ao Departamento de Geografia e História da UNIOESTE, Campus de Marechal Cândido Rondon.

À professora Maria Beatriz Zanchet, pela revisão de português;

A todos que, de uma forma ou de outra, contribuíram para a realização deste trabalho. 


\section{SUMÁRIO}

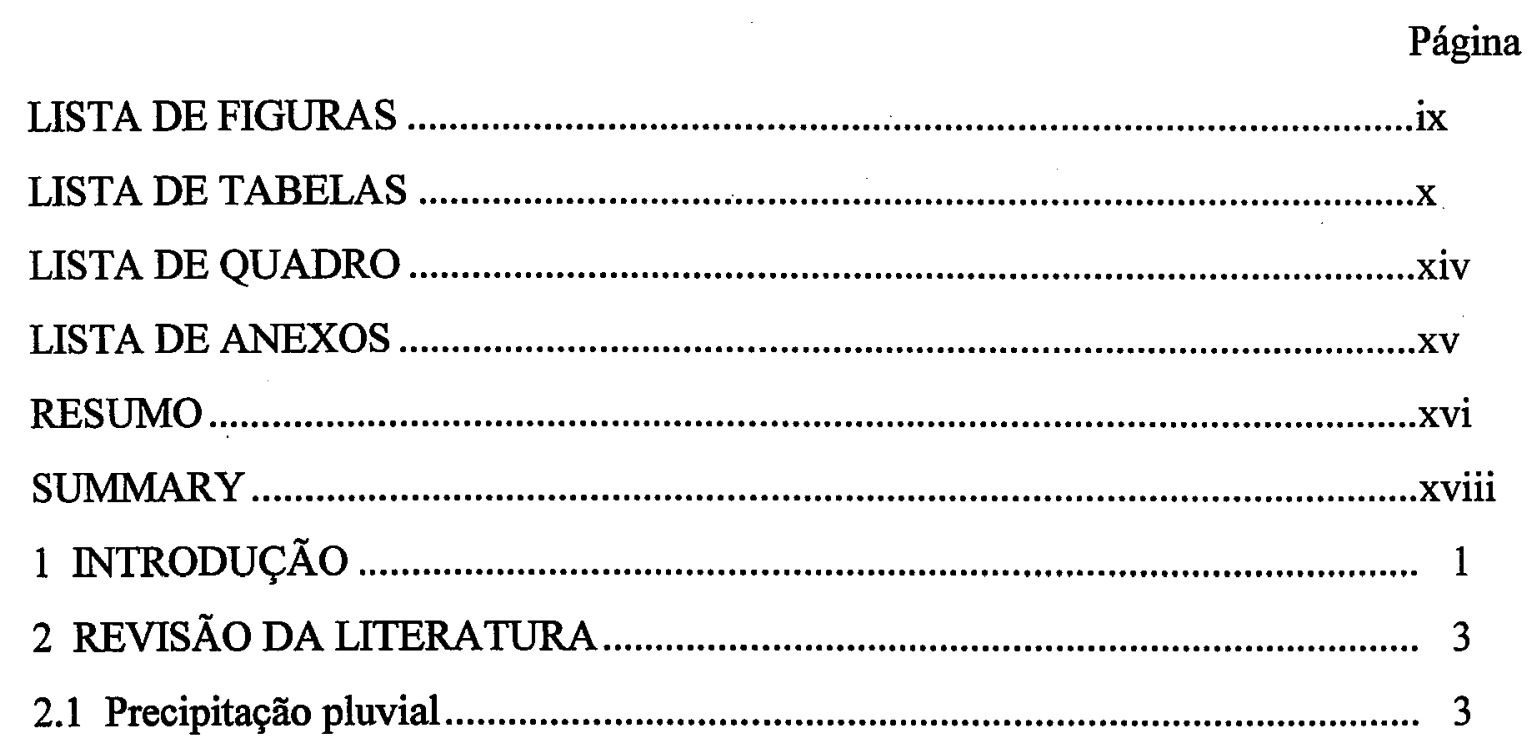

2.2 Classificação climática de Köppen (1918) e Thornthwaite (1948) .................... 6

2.3 Balanço hídrico climático de Thornthwaite e Mather (1955) ............................ 7

3 MATERIAL E MÉTODOS ....................................................................... 9

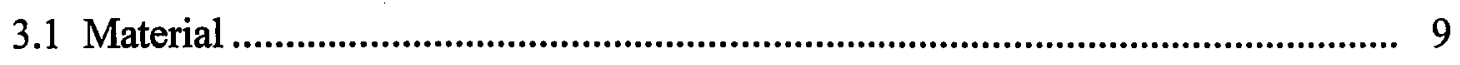

3.1.1 Localização da área de estudo ................................................................. 9

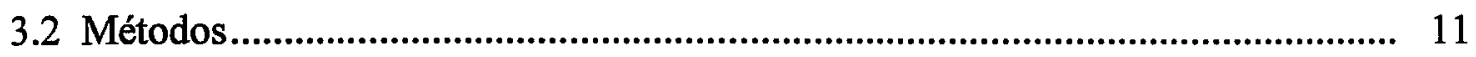

3.2.1 Estimativa de tendências: média móvel .................................................... 11

3.2.2 Classificação climática de Köppen (1918) ............................................... 12

3.2.3 Classificação climática de Thornthwaite (1948)........................................ 14

3.2.4 Balanço hídrico mensal normal, mensal médio e mensal mediano e decendial normal ........................................................................................ 17

3.2.5 Cálculo das freqüiências e probabilidades dos componentes do balanço hídrico seqüencial (Thornthwaite, 1955) na escala mensal. 
3.2.6 Cálculo das frequêencias e probabilidades dos componentes do balanço hídrico seqüencial (Thornthwaite e Mather - 1955) na escala decendial ............... 19

4 RESULTADOS E DISCUSSÕES......................................................... 20

4.1 Classificação climática de Köppen (1918) ...................................................... 20

4.2 Classificação climática de Thornthwaite (1948) .............................................. 24

4.3 Balanço hídrico mensal normal - BHMN e mensal médio (BHMME), mensal mediano (BHMMED) e decendial normal (BHDN) e suas classificações climá-

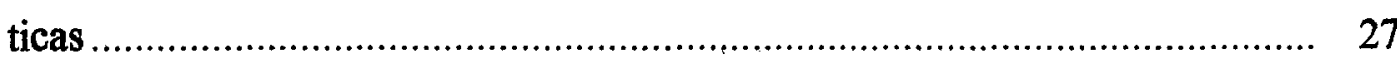

4.4 Variabilidade temporal dos totais anuais pluviométricos e temperaturas médias anuais

4.5 Distribuição mensal e decendial das probabilidades da temperatura média e da precipitação pluvial.

4.6 Distribuição mensal e decendial das probabilidades da evapotranspiração potencial, armazenamento hídrico, evapotranspiração real, deficiência hídrica e excedente hídrico para CAD: 50, 100 e $150 \mathrm{~mm}$

4.7 Uma aplicação agronômica das Tabelas de probabilidades dos componentes do balanço hídrico mensal

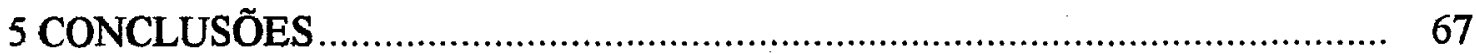

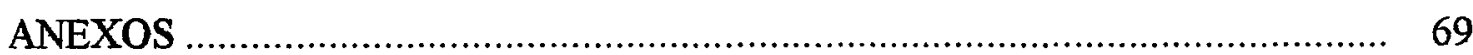

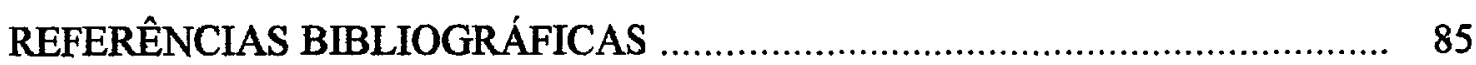




\section{LISTA DE FIGURAS}

Página

1- Localização do município de Piracicaba no Estado de São Paulo

2- Representação esquemática da seqüência de operações, para obtenção da fórmula climática de acordo com o sistema de classificação climática de Köppen, 1918..........13

3- Representação esquemática da seqüência de operações, para obtenção da fórmula climática de acordo com o sistema de classificação climática de Thornthwaite,

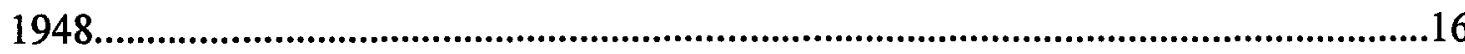

4- Totais pluviométricos anuais da área de estudo. Período: 1917-1996.................. 33

5- Valores da temperatura média anual da área de estudo. Período: 1917-1996 ....... 34 


\section{LISTA DE TABELAS}

Página

1- Distribuição das freqüências dos tipos climáticos de Köppen (1918), nas ordens de $10,15,20,25,30$ e 35 anos na área de estudo (Período: 1917-1996).

2- Distribuição das probabilidades dos tipos climáticos de Köppen (1918), nas ordens de 10, 15, 20, 25, 30 e 35 anos na área de estudo (Período: 1917-1996). 22

3- Distribuição das freqüências dos tipos climáticos de Thornthwaite (1918), nas ordens de 10, 15, 20, 25, 30 e 35 anos na área de estudo (Período: 1917-1996). 24

4- Distribuição das probabilidades dos tipos climáticos de Thornthwaite (1918), nas ordens de $10,15,20,25,30$ e 35 anos na área de estudo (Período: 19171996)

5- Balanço Hídrico Mensal Normal - BHMN (1917-1996) de Piracicaba, segundo Thornthwaite e Mather - 1955 (CAD: $100 \mathrm{~mm}$ )

6- Balanço Hídrico decendial Normal - BHDN (1917-1996) de Piracicaba, segundo Thornthwaite e Mather - 1955 (CAD: $100 \mathrm{~mm}$ ).

7- Balanço Hídrico Mensal Médio - BHMME (1917-1996) de Piracicaba, segundo Thornthwaite e Mather - 1955 (CAD: $100 \mathrm{~mm}$ ).

8- Balanço Hídrico Mensal Mediano- BHMMED (1917-1996) de Piracicaba, segundo Thornthwaite e Mather - 1955 (CAD: $100 \mathrm{~mm}$ ).....

9- Probabilidades (\%) da temperatura média mensal, com 10, 20, 30, 40, 50,60, $70,80,90$, e $99 \%$ de ocorrências na área de estudo entre 1917-1996, com valores menores ou iguais, referentes aos dados abaixo.

10- Probabilidades (\%) da temperatura média decendial, com 10, 20,30,40,50, $60,70,80,90$, e $99 \%$ de ocorrências na área de estudo entre 1917-1996, com valores menores ou iguais, referentes aos dados abaixo 
11- Probabilidades (\%) da precipitação média, com 10, 20,30, 40, 50, 60, 70, 80, 90 , e $99 \%$ de ocorrências, com valores menores ou iguais, referentes aos dados abaixo

12- Probabilidades (\%) da precipitação decendial, com 10, 20, 30, 40, 50,60, 70, 80,90 , e $99 \%$ de ocorrências na área de estudo entre 1917-1996, com valores menores ou iguais, referentes aos dados abaixo.

13- Probabilidades (\%) da evapotranspiração potencial mensal, com 10, 20, 30, 40, $50,60,70,80,90$, e $99 \%$ de ocorrências na área de estudo entre 1917-1996, com CAD: 50,100 e $150 \mathrm{~mm}$, com valores menores ou iguais, referentes aos dados abaixo

14- Probabilidades (\%) da evapotranspiração potencial decendial, com 10, 20,30, $40,50,60,70,80,90$, e $99 \%$ de ocorrências na área de estudo entre 19171996, com CAD: 50mm, com valores menores ou iguais, referentes aos dados abaixo

15- Probabilidades (\%) da evapotranspiração potencial decendial, com 10, 20,30, $40,50,60,70,80,90$, e $99 \%$ de ocorrências na área de estudo entre 19171996, com CAD: $100 \mathrm{~mm}$, com valores menores ou iguais, referentes aos dados abaixo

16- Probabilidades (\%) da evapotranspiração potencial decendial, com 10, 20, 30, $40,50,60,70,80,90$, e $99 \%$ de ocorrências na área de estudo entre 1917 1996, com CAD: $150 \mathrm{~mm}$, com valores menores ou iguais, referentes aos dados abaixo

17- Probabilidades (\%) da evapotranspiração real mensal, com 10, 20, 30, 40, 50, $60,70,80,90$, e $99 \%$ de ocorrências na área de estudo entre 1917-1996, com CAD: 50,100 e $150 \mathrm{~mm}$, com valores menores ou iguais, referentes aos dados abaixo

18- Probabilidades (\%) da evapotranspiração real decendial, com 10, 20,30,40, $50,60,70,80,90$, e $99 \%$ de ocorrências na área de estudo entre 1917-1996, com CAD: $50 \mathrm{~mm}$, com valores menores ou iguais, referentes aos dados abaixo

19- Probabilidades (\%) da evapotranspiração real decendial, com 10, 20,30,40, $50,60,70,80,90$, e $99 \%$ de ocorrências na área de estudo entre 1917-1996, com CAD: $100 \mathrm{~mm}$, com valores menores ou iguais, referentes aos dados abaixo 
20- Probabilidades (\%) da evapotranspiração real decendial, com 10, 20,30,40, $50,60,70,80,90$, e $99 \%$ de ocorrências na área de estudo entre 1917-1996, com CAD: $150 \mathrm{~mm}$, com valores menores ou iguais, referentes aos dados abaixo

21- Probabilidades (\%) do armazenamento hídrico mensal, com 10, 20,30, 40, 50, $60,70,80,90$, e $99 \%$ de ocorrências para as CAD: 50, 100 e $150 \mathrm{~mm}$, com valores menores ou iguais, referentes aos dados abaixo

22- Probabilidades (\%) do armazenamento hídrico decendial, com 10, 20,30,40, $50,60,70,80,90$, e $99 \%$ de ocorrências para a CAD: $50 \mathrm{~mm}$, com valores menores ou iguais, referentes aos dados abaixo

23- Probabilidades (\%) do armazenamento hídrico decendial, com 10, 20, 30, 40, $50,60,70,80,90$, e $99 \%$ de ocorrências para a CAD: $100 \mathrm{~mm}$, com valores menores ou iguais, referentes aos dados abaixo.

24- Probabilidades (\%) do armazenamento hídrico decendial, com 10, 20,30,40, $50,60,70,80,90$, e $99 \%$ de ocorrências para a CAD: $150 \mathrm{~mm}$, com valores menores ou iguais, referentes aos dados abaixo

25- Probabilidades (\%) da deficiência hídrica mensal, com 10, 20,30,40,50,60, $70,80,90$, e $99 \%$ de ocorrências para as CAD: 50,100 e $150 \mathrm{~mm}$, com valores menores ou iguais, referentes aos dados abaixo

26- Probabilidades (\%) da deficiência hídrica decendial, com 10,20,30,40, 50, $60,70,80,90$, e $99 \%$ de ocorrências para a CAD: $50 \mathrm{~mm}$, com valores menores ou iguais, referentes aos dados abaixo

27- Probabilidades (\%) da deficiência hídrica decendial, com 10,20,30,40,50, $60,70,80,90$, e $99 \%$ de ocorrências para a CAD: $100 \mathrm{~mm}$, com valores menores ou iguais, referentes aos dados abaixo

28- Probabilidades (\%) da deficiência hídrica decendial, com 10,20,30,40,50, $60,70,80,90$, e $99 \%$ de ocorrências para a CAD: $150 \mathrm{~mm}$, com valores menores ou iguais, referentes aos dados abaixo.

29- Probabilidades (\%) do excedente hídrico mensal, com 10,20,30,40,50,60, $70,80,90$, e $99 \%$ de ocorrências para as CAD: $50,100,150 \mathrm{~mm}$, com valores menores ou iguais, referentes aos dados abaixo.

30- Probabilidades (\%) do excedente hídrico decendial, com 10,20,30,40,50,60, $70,80,90$, e $99 \%$ de ocorrências para a CAD: $50 \mathrm{~mm}$, com valores menores ou iguais, referentes aos dados abaixo 
31- Probabilidades (\%) do excedente hídrico decendial, com 10, 20, 30, 40, 50, 60, $70,80,90$, e $99 \%$ de ocorrências para a CAD: $100 \mathrm{~mm}$, com valores menores ou iguais, referentes aos dados abaixo

32- Probabilidades (\%) do excedente hídrico decendial, com 10, 20, 30, 40,50,60, $70,80,90$, e $99 \%$ de ocorrências para a CAD: $150 \mathrm{~mm}$, com valores menores ou iguais, referentes aos dados abaixo 


\section{LISTA DE QUADRO}

Página

1- Quebra de produtividade e rendimento da cultura do milho, com probabilidades de $70 \%$ de ocorrências dos valores mensais da evapotranspiração potencial e deficiência hídrica. 


\section{LISTA DE ANEXOS}

Página

1 ANEXO A- Quadro 1: Classificação Climática de Köppen para a classe de 10 anos (Período: 1917-1996).

2 ANEXO A- Quadro 2: Classificação Climática de Köppen para a classe de 15 anos (Período: 1917-1996)

3 ANEXO A- Quadro 3: Classificação Climática de Köppen para a classe de 20 anos (Período: 1917-1996)

4 ANEXO A- Quadro 4: Classificação Climática de Köppen para a classe de 25 anos (Período: 1917-1996)

5 ANEXO A- Quadro 5: Classificação Climática de Köppen para a classe de 30 anos (Período: 1917-1996)

6 ANEXO A- Quadro 6: Classificação Climática de Köppen para a classe de 35 anos (Período: 1917-1996)

7 ANEXO B- Quadro 1: Classificação Climática de Thornthwaite para a classe de 10 anos.

8 ANEXO B- Quadro 2: Classificação Climática de Thornthwaite para a classe de 15 anos.

9 ANEXO B- Quadro 3: Classificação Climática de Thornthwaite para a classe de 20 anos.

10 ANEXO B- Quadro 4: Classificação Climática de Thornthwaite para a classe de 25 anos

11 ANEXO B- Quadro 5: Classificação Climática de Thornthwaite para a classe de 30 anos.

12 ANEXO B- Quadro 6: Classificação Climática de Thornthwaite para a classe de 35 anos. 


\title{
VARIAÇÃO TEMPORAL DO BALANÇO HÍDRICO E DO CLIMA, DE ACORDO COM OS CRITÉRIOS DE KÖPPEN (1918) E THORNTHWAITE (1948), NA REGIÃO PIRACICABA - SP
}

\author{
Autora: ZILDA DE FÁTIMA MARIANO
}

Orientador: Prof. Dr. VALTER BARBIERI

RESUMO

O presente trabalho teve por objetivo determinar o número mínimo de anos adequados para identificar o tipo climático e as flutuações entre seus limites pelo método de classificação de Köppen (1918) e de Thornthwaite (1948), avaliar as probabilidades dos componentes do balanço hídrico de Thornthwaite e Mather (1955) na escala mensal e decendial e comparar os balanços hídricos normal, médio e mediano visando oferecer informações mais completas para uma análise climática e planejamento das atividades da agricultura. Para tanto, foram utilizadas 80 anos de dados mensais e decendiais de temperatura do ar e precipitação pluvial de Piracicaba-SP. O método utilizado foi a classificação climática de Köppen (1918) e de Thornthwaite (1948), o programa computacional do balanço hídrico (Barbieri et al., 1991) e freqüências e probabilidades dos componentes do balanço hídrico. A partir dos resultados das freqüências e probabilidades da classificação climática de Köppen, para as médias móveis nas ordens de 10,15, 20, 25, 30 e 35 anos identificou-se que o tipo climático foi Cfa (clima tropical chuvoso, com a precipitação do mês mais úmido menor que 10 vezes do mês mais seco) com transição para Cwa (clima tropical chuvoso, com a precipitação do mês mais úmido igual ou maior que 10 vezes do mês mais seco). Para a classificação de Thornthwaite a região enquadrou-se no tipo climático $\mathrm{B}_{1} \mathrm{r}^{\prime} \mathrm{B}_{4} \mathrm{a}^{\prime}$ (tipo úmido, com o Índice efetivo de umidade ( $\mathrm{Im}$ ) entre 20 a 40\%) com transição para $\mathrm{C}_{2} \mathrm{r}$ $\mathrm{B}_{4}{ }_{4} \mathrm{a}^{\prime}$ (tipo subúmido, Im entre 20 a $0 \%$ ), os quais possuem pequena ou nenhuma deficiência de água, mesotérmicos, em que $48 \%$ da estação de 
crescimento das plantas se concentram no verão. Identificou-se que 10 anos de observação seria suficiente para identificar o clima de Piracicaba com $\mathrm{B}_{1} \mathrm{r} \mathrm{B}_{4}{ }_{4} \mathrm{a}^{\prime}$ (Thornthwaite) pois apresentou $70 \%$ de probabilidades de ocorrências e como Cfa ou Cwa segundo Köppen, pois as probabilidades foram próximas $48 \%$ e $51 \%$ respectivamente, sendo mais preciso a classificação com 15 anos em que as probabilidades foram de $58 \%$ para o tipo Cfa. Existe uma certa tendência dos meses úmidos (DEZ, JAN e FEV) tornarem-se menos úmidos e os meses secos (JUN, JUL e AGO) tornarem-se menos secos, ou seja, um aumento das precipitações no inverno e diminuição no verão. O balanço hídrico normal, médio e mediano apresentaram o mesmo tipo climático pelo método de Thornthwaite $B_{1} r B_{4}^{\prime} a^{\prime}$, enquanto pelo método de Köppen o tipo foi Cfa para o balanço hídrico normal e médio e o tipo Cwa para o balanço hídrico mediano. As freqüências e probabilidades de ocorrência dos elementos do clima e componentes do balanço hídrico permitem um análise mais precisa do clima da regiâo de Piracicaba. O balanço hídrico normal decendial tem seus componentes iguais a $1 / 3$ dos componentes do balanço hídrico normal mensal, indicando que uma análise climática tem a mesma precisão utilizando-se valores mensais ou decendiais. As probabilidades dos componentes se forem utilizados do balanço hídrico para as diferentes CADs permitem análises agroclimáticas com relativa precisão. 
BALANÇO HIDRICO'S TEMPORARY VARIATION AND OF THE CLIMATE, IN AGREEMENT WITH THE APPROACHES OF KÖPPEN (1918) AND THORNTHWAITE (1948), IN THE REGIÃO PIRACICABA - SP

\author{
Author: ZILDA DE FÁTIMA MARIANO
}

Adviser: Prof. Dr. VALTER BARBIERI

\title{
ABSTRACT
}

The present work had for objective to determine the minimum number of years adapted to identify the climatic type and the flotations among its limits for the method of classification of Köppen (1918) and of Thornthwaite (1948), to evaluate the probabilities of the components of the swinging hidrico of Thornthwaite and Mather (1955) in the monthly scale and decendial and to compare the swingings normal, medium and medium hidricos seeking to offer more complete information for a climatic analysis and planning of the activities of the agriculture. For so much, 80 years of monthly data and decendiais of temperature of the air and pluvial precipitation of Piracicaba-SP were used. The used method went to climatic classification of Köppen (1918) and of Thornthwaite (1948), the program computacional of the swinging hídrico (Barbieri et al., 1991) and frequencies and probabilities of the components of the swinging hidrico. Starting from the results of the frequencies and probabilities of the climatic classification of Köppen, for the mobile averages in the orders of $10,15,20,25,30$ and 35 years he/she identified that the climatic type was Cfa (rainy tropical climate, with the precipitation of the smaller most humid month than 10 times of the driest month) with transition for Cwa (rainy tropical climate, with the precipitation of the month most humid equal or larger than 10 times of the driest month). Para the classification of Thornthwaite the area enquadrou-if in the climatic type B1 r B'4 a' (humid type, with the effective Index of humidity (Im) among 20 to $40 \%$ ) with transition for C2 r B'4 a' (type subúmido, Im among 20 to $0 \%$ ), which possess small or any deficiency of water, mesoterrmicos, in that $48 \%$ of the station of growth of the plants concentrate on the summer. He/she identified that 10 years of observation it would be enough to identify the climate of Piracicaba with B1 r B'4 a' (Thornthwaite) because it 
presented $70 \%$ of probabilities of occurrences and like Cfa or Cwa according to Köppen, because the probabilities were respectively next $48 \%$ and $51 \%$, being more necessary the classification with 15 years in that the probabilities were of $58 \%$ for the type $\mathrm{Cfa}$. A certain tendency of the humid months exists (TEN, JAN and FEV) they turn her less humid and the dry months (JUN, JUL and AGO) they turn her less dry, that is to say, an increase of the precipitations in the winter and decrease in the summer. The swinging normal, medium and medium hidrico presented the same climatic type for the method of Thornthwaite B1 $r$ $B^{\prime} 4 a^{\prime}$, while for the method of Köppen the type went Cfa to the swinging normal and medium hídrico and the type Cwa for the swinging medium hídrico. The frequencies and probabilities of occurrence of the elements of the climate and components of the swinging hídrico allow a more precise analysis of the climate of the area of Piracicaba. The swinging hídrico normal decendial has its components the same to $1 / 3$ of the components of the swinging monthly normal hídrico, indicating that a climatic analysis has the same precision being used monthly values or decendiais. The probabilities of the components if they be used of the swinging hídrico for different CADs they allow analyses agroclimáticas with relative precision. 


\section{INTRODUÇÃO}

Independentemente dos recentes avanços tecnológicos e científicos na agricultura, o clima é um dos fatores importantes na determinação da viabilidade econômica do cultivo de certas culturas em uma dada região. Conforme Köppen (1931) entende-se por clima o estado médio do tempo de um determinado lugar. Assim, o clima é a síntese dos estudos de tempos atmosféricos, ou seja, é a atuação dos estados atmosféricos alternados e dos elementos meteorológicos, num dado lugar, durante determinado período. Segundo Ayoade (1986), o período sugerido pela Organização Mundial de Meteorologia (OMM) é de no mínimo, 30 anos.

Dentre os elementos meteorológicos mais utilizados para o conhecimento do clima estão a temperatura do ar e a precipitação pluvial, dada sua importância e maior facilidade de serem coletados. Köppen (1918) baseia a classificação climática do mundo nesses dois elementos meteorológicos e no tipo de vegetação. Para o mesmo propósito, Thornthwaite (1948) parte desses elementos e inclui o conceito de evapotranspiração potencial. Essas duas classificações climáticas são baseadas em índices, que por sua vez, determinam limites para cada tipo climático.

Os tipos climáticos, determinados por índices possuem limites fechados, restringindo a classificação climática de uma região. Tais limites fechados classificam o clima numa escala regional não considerando os sistemas locais, ou seja, pontuais.

Assim; classificar um clima é algo difícil, pois tem-se que considerar a atuação dos estados médios atmosféricos e dos elementos meteorológicos, especialmente quando 
influenciada pelas atuações dos diferentes sistemas atmosféricos, as massas tropicais, as massas polares e sistemas frontais, responsáveis pela grande variabilidade nos elementos meteorológicos.

Dessa forma; objetivou-se determinar o número mínimo de anos adequados para identificar o tipo climático $\mathrm{e}$ as flutuações entre seus limites pelo método de classificação climática de Köppen e de Thornthwaite; avaliar as probabilidades dos componentes do balanço hídrico de Thornthwaite e Mather (1955) na escala mensal e decendial, no período de 1917 a 1996 e comparar o balanço hídrico mensal normal elaborado com temperatura e precipitação médias com o balanço hídrico médio de 80 anos e o balanço hídrico elaborado com os valores medianos de temperatura e precipitação, visando oferecer informações mais completas para uma análise climática e planejamento das atividades da agricultura. 


\section{REVISÃO DA LITERATURA}

Para caracterizar o clima de uma determinada região é importante constatar os elementos que mais influenciam o local. Dentre os elementos meteorológicos, os mais utilizados para a classificação do clima são a temperatura do ar e a precipitação pluvial. A temperatura do ar possui, nos trópicos, uma certa estabilidade, ou seja, sua variabilidade é muito pequena de ano para ano, enquanto a precipitação pluvial apresenta grande variabilidade temporal e espacial nas regiões tropicais e de transição climática como é região de Piracicaba, objeto de estudo deste trabalho. A definição do clima se baseia no volume e na distribuição da precipitação, sendo estes os elementos principais de análise para a caracterização climática de um determinado lugar.

\subsection{Precipitação Pluvial}

Em se tratando de regiões tropicais, a precipitação pluvial assume maior variabilidade em sua distribuição (Azevedo, 1974). Quanto às variações dos totais pluviométricos em regiões tropicais, estas controlam todo um conjunto de atividades agrícolas e hidrológicas, das quais tantas outras dependem, sendo afetadas pelas secas ou enchentes, decorrentes de redução ou aumento das chuvas (Galvani, 1996). Assim, em qualquer estudo sobre precipitação pluvial, existe a necessidade de se distinguir o número de dias chuvosos daqueles não chuvosos e qual o período de ocorrência (Assis, 1991).

Os totais pluviométricos anuais do Estado de São Paulo são provenientes, principalmente, dos sistemas frontais. Tais sistemas segundo Monteiro (1973) são 
responsáveis pelas chuvas de inverno e outono e Tarifa (1975) confirma este fato, sendo estes responsáveis por $67 \%$ das precipitações ocorridas nas estações da primavera e verão.

Pinto et al. (1989) classificam a variação da chuva, no Estado de São Paulo, em três setores: mais chuvoso, região serrana e nordeste; intermediário, região centro-oeste, sudoeste e leste, na qual se encontra Piracicaba e menos chuvoso, região oeste.

Braga Júnior et al. (1991) enfatizam que a distribuição espacial das precipitações no Estado de São Paulo possui sazonalidade bem definida em dois períodos, sendo chuvoso de outubro a março, com $80 \%$ do número total de dias, com chuva e menos chuvoso de abril a setembro, com respectivamente, $20 \%$.

Sant'Anna Neto (1995), em estudo realizado sobre o Estado de São Paulo, no período compreendido entre 1941 e 1993, constatou que os três meses consecutivos de maior pluviosidade são os meses de novembro a janeiro ou dezembro a fevereiro e os de menor pluviosidade maio a julho ou junho a agosto. Nas últimas décadas observadas, a maior ocorrência de chuvas se deu entre os meses de dezembro a fevereiro ou janeiro a março; inversamente, a menor ocorrência situou-se entre os meses de junho a agosto ou julho a setembro, adentrando a fase de plantio da maioria das culturas produzidas no Estado.

Santos (1996), através da análise de tendência semi-média, para a série temporal de 1941 a 1993, aponta os índices de aumento e diminuição das chuvas para o Estado de São Paulo, mostrando a redução das chuvas para o Litoral e Planalto Atlântico (Ubatuba) com coeficiente de variabilidade de 20\%, Vale do Paraiba (Paraibuna) 22\% e Mantiqueira (Campos do Jordão) 16\%; equilíbrio na porção central do Litoral paulista de $19 \%$ e aumento para o restante do espaço paulista entre 17 a $23 \%$.

Estudos realizados na bacia do rio Piracicaba (Christofoletti, citado por Pellegrino, 1995) constataram que a precipitação pluvial do mês mais seco é inferior a $20 \mathrm{~mm}$ e a do mês mais úmido gira em torno de $300 \mathrm{~mm}$, sendo o verão responsável por $70 \%$ do volume de precipitação.

O município de Piracicaba (Estado de São Paulo) encontra-se influenciado pelas massas tropicais, pelas massas polares e sistemas frontais, resultando em grande 
variabilidade da precipitação pluvial em relação a sua distribuição em quantidade, intensidade e duração ao longo dos anos, meses e dias (Piccinini, 1993; Assis, 1991; Ometto, 1991, Galvani, 1997).

Sansigolo (1991), objetivando uma análise agroclimática, estudou as precipitações diárias de Piracicaba para as 73 pêntadas (cinco em cinco dias) do ano, constatando que precipitações entre os valores de 50 a $60 \mathrm{~mm}$ ocorreram no verão, $40 \mathrm{a}$ $20 \mathrm{~mm}$ no outono e primavera e menor de $15 \mathrm{~mm}$ no inverno. Tais ocorrências alcançaram $80 \%$ de probabilidade.

Martorano et al. (1997), aplicando a medida de tendência das médias móveis para a precipitação pluvial em Piracicaba (1917-1996), constataram que na estação do inverno e do outono, as chuvas se normalizaram na ordem de 10 anos, enquanto na primavera e verão se normalizaram a partir da ordem de 20 anos.

Galvani (1997), utilizando-se da regressão linear simples para análise da tendência anual e mensal dos totais pluviométricos de Piracicaba (1917-1994), verificou que a chuva média anual foi de $1274,4 \mathrm{~mm}$, e que no período entre abril e setembro foi abaixo de $100 \mathrm{~mm}$, representando $22,5 \%$ do total médio anual; entre outubro e março ficou acima de $100 \mathrm{~mm}$, perfazendo $77,5 \%$. A tendência anual das chuvas para o período total de 1917 a 1994 foi crescente na ordem de 129,5 mm porém, registrou-se uma tendência decrescente em relação ao periodo mensal total nos meses de janeiro e dezembro, os quais apresentaram, 19,3 $\mathrm{mm}$ e $36,0 \mathrm{~mm}$, respectivamente.

\subsection{Classificação climática de Köppen (1918) e Thornthwaite (1948)}

As inúmeras variações no tempo, de local para local, determinadas por diferentes combinações dos processos atmosféricos, resultam em diferentes tipos climáticos (Vianello e Alves, 1991). Para identificar esses tipos climáticos é necessário classificálos em áreas homogêneas. Assim, a classificação climática possui duas funções básicas: a primeira é a identificação e organização dos dados médios dos elementos meteorológicos em determinado local, definindo os limites de influência a segunda é a 
utilização desses limites (mapeamento) para a organização das atividades humanas (Virmani et al., 1980).

Os elementos meteorológicos necessários para a classificação climática dependem da finalidade a que se destinam. Dessa forma, segundo Toledo (1973), várias classificações climáticas com diferentes critérios, foram propostas: Hann (1883); Lang (1920); Köppen (1918, 1920); De Martonne (1926, 1936, 1942); Joly (1947); Thornthwaite (1931, 1948, 1955); Gaussen \& Bagnouls (1952); Mohre \& Boreu (1954). Entretanto, as duas mais utilizadas mundialmente são as de Köppen (1918) e Thornthwaite (1948).

O modelo de classificação climática de Köppen (1918) baseia-se nos seguintes elementos meteorológicos: temperatura do ar, precipitação pluvial e vegetação. Através destes elementos, identificam-se as regiões climáticas e suas fronteiras em escala global.

O modelo de classificação de Thornthwaite (1948) baseia-se no modelo de Köppen, porém utilizando dados de hidrologia, solos e evapotranspiração potencial, pois somente os valores pluviométricos não especificam um clima como seco ou úmido, sendo necessário conhecer, também, as suas necessidades hídricas em escala regional,

adicionando a este modelo o balanço hídrico com Capacidade de Água Disponível (CAD) $100 \mathrm{~mm}$. A classificação climática de Thornthwaite fundamenta-se em dois parâmetros: o índice efetivo de umidade e o índice de eficiência térmica, os quais são funções diretas da evapotranspiração potencial (Nimer, 1989; Ayoade, 1986 e Köppen, 1931).

Dessa forma, a classificação climática é a síntese e agrupamento dos elementos meteorológicos em tipos climáticos, a partir dos quais as regiões são mapeadas.

\subsection{Balanço hídrico climático de Thornthwaite \& Mather (1955)}

O conhecimento do balanço hídrico de uma região é fundamental para qualquer estudo de planejamento e gestão de recursos hídricos. $O$ balanço hídrico é um sistema contábil de monitoramento da água no solo e resulta da aplicação do princípio de 
conservação de massa de água num volume de solo vegetado (Pereira et al., 1997).

Thornthwaite \& Mather citado por Pereira et a1., 1997, descrevem o balanço hídrico climatológico como sendo uma das diversas maneiras de se monitorar o armazenamento de água no solo, em que o suprimento de água natural é a precipitação pluvial, a demanda atmosférica é a evapotranspiração potencial e a Capacidade de Àgua Disponivel (CAD) é apropriada ao tipo de cultura.

A Capacidade de Água Disponível (CAD) é a quantidade de água existente num dado perfil de solo entre a Capacidade de Campo (CC) e o ponto de murcha permanente (PMP), a qual varia conforme a estrutura do solo e a profundidade das raízes. Os solos arenosos retêm menor quantidade de água em relação aos solos argilosos. Conforme Camargo (1978), as raizes das hortaliças exploram uma camada superficial do solo, na qual o armazenamento é inferior a $100 \mathrm{~mm}$; para as plantas agrícolas (algodão, milho, mamona, e outras) o limite é de $100 \mathrm{~mm}$ e para vegetações arbóreas e vigorosas, como eucaliptus, citrus e outras, o limite é superior a $100 \mathrm{~mm}$.

$\mathrm{Na}$ Índia, o método do balanço hídrico de Thornthwaite \& Mather está sendo utilizado para determinar a produtividade nas regiões, considerando as deficiências hídricas e excessos hídricos (Srivastava et al., 1996; Nathan \& Sinha, 1996). Este método vem sendo cada vez mais utilizado, tanto no Brasil (Santos et al., 1976; Camargo, 1978; Barbieri et al., 1991; Braga.Júnior et al., 1991; Tommaselli, 1992; Martorano et al., 1993, Martorano et al.,1997 e outros) quanto em outras partes do mundo.

Camargo (1978), através do estudo do balanço hídrico do Estado de São Paulo, concluiu que, na região de Piracicaba, a evapotranspiração potencial anual encontrou-se na faixa mesotérmica (1000-1100 $\mathrm{mm}$ ) e que as temperaturas e as necessidades hídricas são moderadas, sendo do tipo climático mesotérmico $\left(\mathrm{B}_{3^{\prime}}{ }^{\prime}=750\right.$ a $\left.1100 \mathrm{~mm}\right)$. Os excessos hídricos médios anuais foram de $300 \mathrm{~mm}$ e as deficiências hídricas médias anuais de $40 \mathrm{~mm}$.

Para facilitar o cálculo do balanço hídrico Barbieri et al. (1991), baseados no balanço hídrico de Thornthwaite \& Mather (1955), elaboraram um programa computacional, escrito na linguagem BASIC, o qual utiliza os dados médios mensais de 
precipitação pluvial e temperatura do ar, para o cálculo do balanço hídrico seqüencial mensal, que representa a ocorrência mais provável das condições climáticas da região e dados decendiais (10 dias) para o balanço hídrico seqüencial decendial representando as condições hídricas. Este programa foi utilizado entre outros, por Martorano et al. (1997), Moraes (1998), Martorano (1998) e Santos (1998). 


\section{MATERIAL E MÉTODOS}

\subsection{Material}

\subsubsection{Localização da área de estudo}

A área de estudo é o município de Piracicaba, delimitada pelas seguintes coordenadas geográficas: latitude $22^{\circ} 42^{\prime} 30^{\prime \prime} \mathrm{S}$ e longitude $47^{\circ} 38^{\prime} 01^{\prime \prime} \mathrm{W}$. O município situa-se na quinta $\left(5^{\mathrm{a}}\right)$ região Política Administrativa, sendo o décimo nono em extensão territorial do Estado de São Paulo, ilustrado na Figura 1.

A geomorfologia da área apresenta uma altitude entre 400 a $800 \mathrm{~m}$ está inserida na Depressão Periférica, zona do Médio Tietê, sendo geologicamente $70 \%$ da área pertence ao Triássico-Jurássico e $30 \%$ ao Permiano. Os solos predominante são Latossolos e Podzólicos e em menor extensão os solos Litólicos e Terra Roxa Estruturada, (DAEE, 1973).

A série temporal de dados diários e mensais de precipitação pluvial e temperatura do ar de 80 anos (1917-1996) foi fornecida pelo Departamento de Física e Meteorologia, da "Escola Superior de Agricultura Luiz de Queiroz", Universidade de São Paulo, ESALQ/USP. Até 1945, a estação meteorológica situava-se próxima ao Instituto Zimotécnico, a latitude de $22^{\circ} 42^{\prime} 30^{\prime \prime} \mathrm{S}$, longitude $47^{\circ} 38^{\prime} 00^{\prime}$ ' W e $546 \mathrm{~m}$ (Assis, 1991). A partir dessa data, a estação foi transferida a $2 \mathrm{Km}$ de distância para latitude $22^{\circ} 42^{\prime} 10^{\prime \prime} \mathrm{S}$, longitude $47^{\circ} 37^{\prime} 21$ " W e $564 \mathrm{~m}$, localidade atual. 


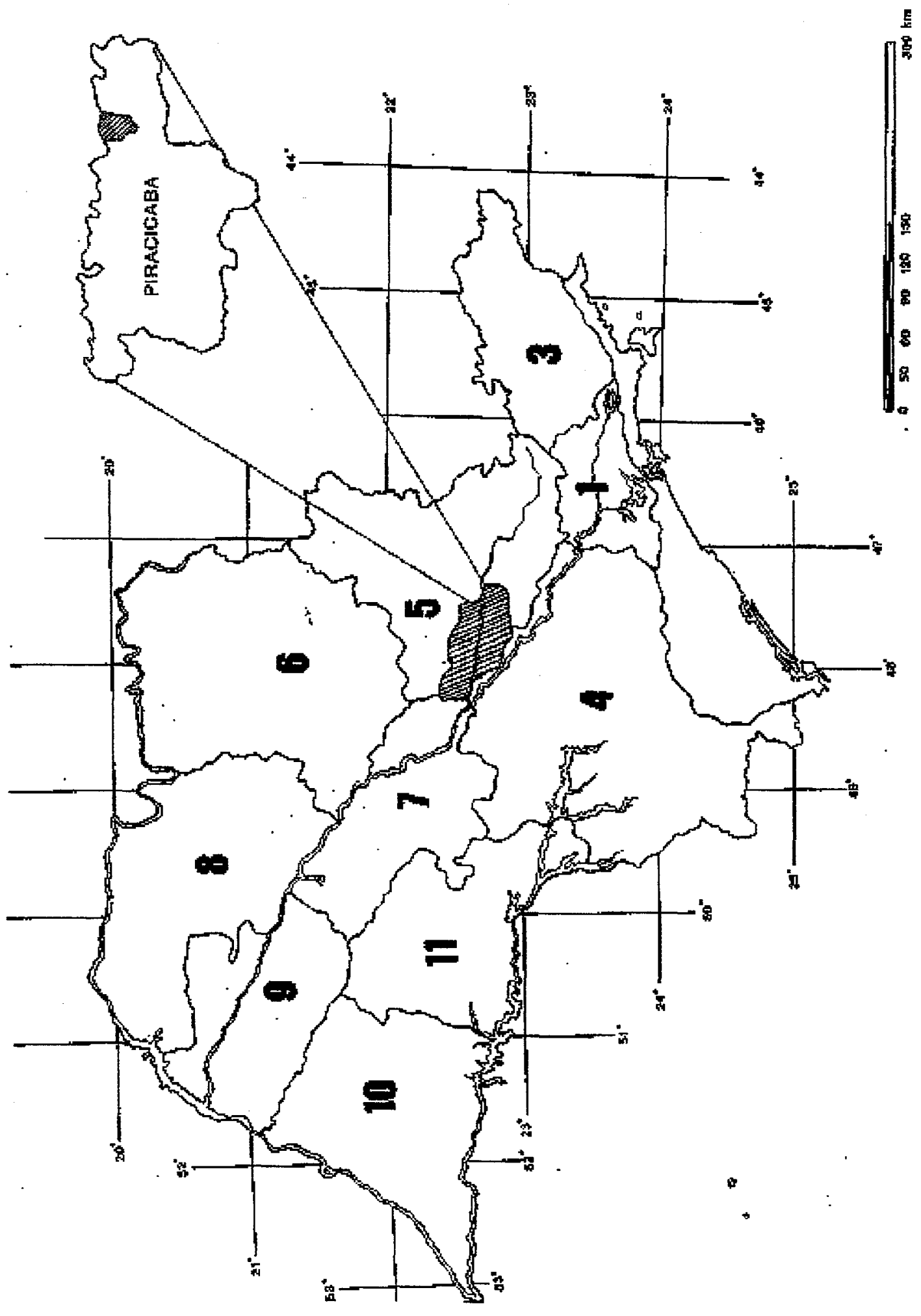

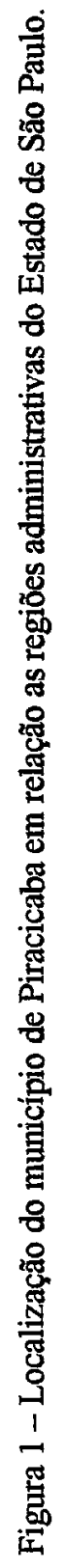




\subsection{Métodos}

\subsubsection{Estimativa de tendência: média móvel}

Para os dados mensais de precipitação pluvial e temperatura média do ar do período de 1917 a 1996, foi calculada estatisticamente, a estimativa de tendência: média móvel para as ordens de $10 ; 15 ; 20 ; 25 ; 30$ e 35 anos, conforme a equação 1 .

$$
\left.\bar{X}_{\text {móvel }}=x_{j}+x_{j+1}+x_{j+2}+\ldots+x_{j+k}\right) / k+1
$$

em que: $\bar{X}_{\text {móvel }}=$ média móvel;

$$
\begin{aligned}
& x_{j}=\text { ano referente }(1917 \ldots 1996) \\
& \mathrm{k}=\text { número de ordem }(9+1=10 \text { anos; } 11+4=15 \text { anos....31+4= } 35 \text { anos }) .
\end{aligned}
$$

\subsubsection{Classificação climática de Köppen (1918)}

A partir dos cálculos das médias móveis para cada ordem, obtidos a partir da eq. 1, aplica-se o modelo de classificação climática de Köppen, o qual baseia-se na temperatura média do ar, precipitação pluvial e vegetação.

A classificação climática de Köppen parte dos seguintes critérios:

a) Critério térmico: a temperatura média do mês mais frio classifica a superfície do globo terrestre em tipos climáticos, designados por letras maiúsculas, em que o tipo A é determinado quando a temperatura média é maior ou igual a $18,0^{\circ} \mathrm{C}$ e o tipo $\mathrm{C}$ quando a temperatura média está entre $18,0^{\circ} \mathrm{C}$ até $-3,0^{\circ} \mathrm{C}$ e outros como o tipo $\mathrm{B}$ que apresenta 
potencial média anual que é maior do que a precipitação média anual, não ocorrendo excedente hídrico, o que corresponde a um clima seco. Para a região de Piracicaba, somente estes dois tipos climáticos, $\mathrm{A}$ e C, são necessários para determinar o tipo de clima.

b) Critério estacional: a distribuição estacional das precipitações subdivide os tipos climáticos em subtipos designados em letras minúsculas: w e f; em que o subtipo w é determinado quando a precipitação do mês mais úmido é igual ou maior que 10 vezes a do mês mais seco, e o subtipo f é quando a precipitação do mês mais úmido é menor que 10 vezes a do mês mais secco.

c) Critério adicional de temperatura: a característica adicional da temperatura, expressa pela letra minúscula a, é acrescentada quando a temperatura média do mês mais quente for maior ou igual a $22,0^{\circ} \mathrm{C}$. Esses critérios estão apresentados na figura 1 .

\subsubsection{Classificação climática de Thornthwaite (1948)}

A partir do programa computacional do balanço hídrico, realizado por Barbieri et al. (1991), baseado no conceito de evapotranspiração potencial de Thornthwaite (1948) e no modelo de Thornthwaite e Mather (1955), calcularam-se os balanços hídricos, com Capacidade de Água Disponível (CAD) de $100 \mathrm{~mm}$ para cada ordem (médias móveis: $10 ; 15 ; 20 ; 25 ; 30$ e 35 anos) aplicando-se o modelo da classificação climática de Thornthwaite (1948).

O modelo de classificação climática de Thornthwaite baseia-se nos mesmos elementos climáticos da classificação de Köppen, mas introduz o cálculo da evapotranspiração potencial como elemento de classificação climática, relacionando o com as necessidades hídricas da região, conceito este presente no modelo do balanço hídrico de Barbieri et al. (1991) o qual é determinado pelas seguintes equações: 


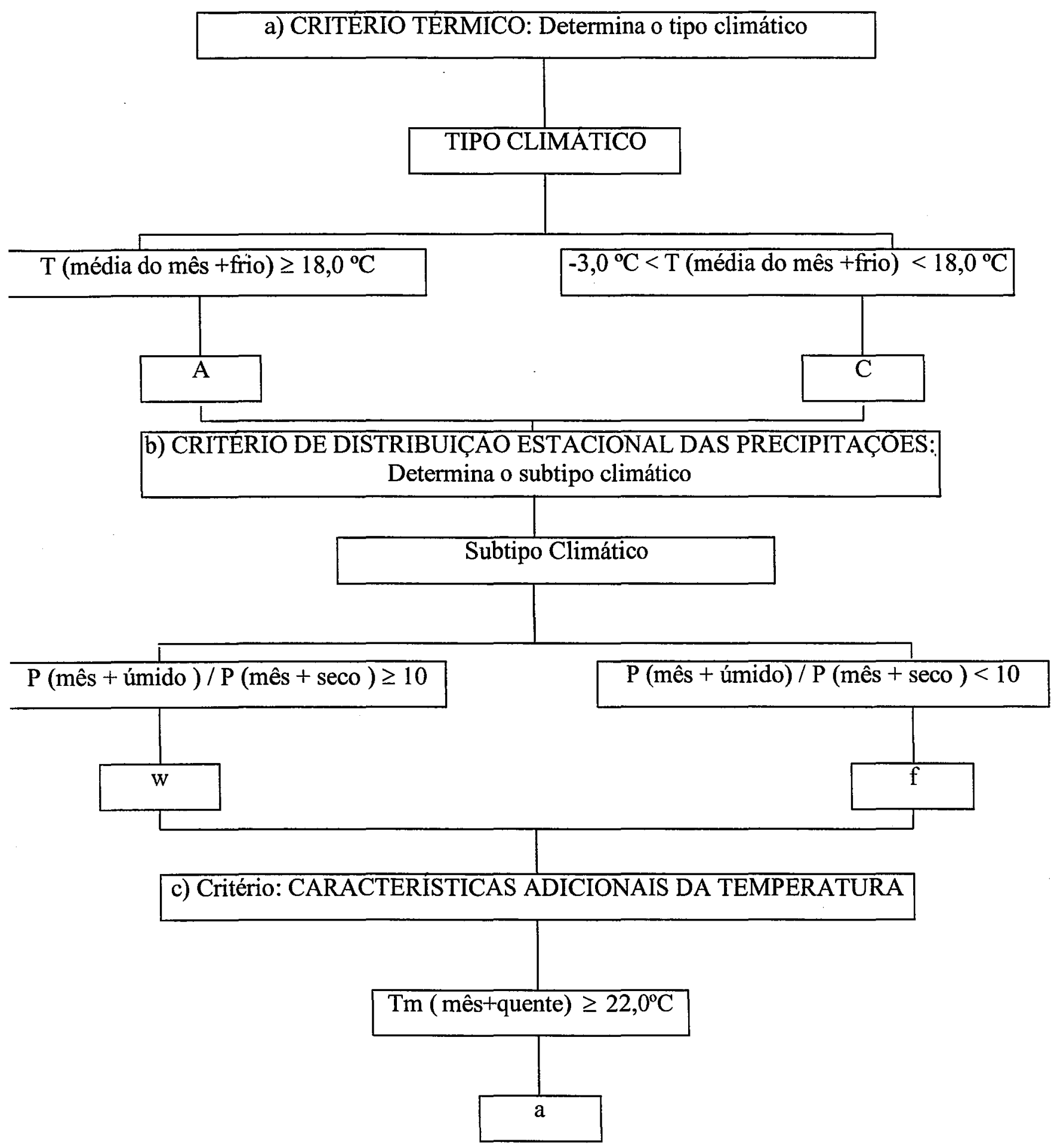

Figura 2: Representação esquemática da seqüência de operações, para obtenção da fórmula climática de acordo com o sistema de classificação climática de Köppen, 1918. 


$$
E T P=16\left(10 \frac{T i}{I}\right)^{a} \quad T i>0^{\circ} C
$$

em que: ETP= evapotranspiração potencial média mensal padrão (mm.mês $\left.{ }^{-1}\right)$;

$\mathrm{Ti}=$ temperatura média mensal $\left({ }^{\circ} \mathrm{C}\right)$,

I = índice de calor mensal da região, calculado com os valores normais de temperatura.

$$
a=6,75 * 10^{-7} * I^{3}-7.71 * 10^{-5} * \mathrm{I}^{2}+0,01792 * I+0,49239
$$

em que: a = coeficiente empírico, calculado em função do índice de calor mensal da região;

I = índice de calor anual da região, calculado com os valores normais de temperaturas mensais.

$$
I=\sum_{i=1}^{12}(0,2 \quad T i)^{1,514} \quad T i>0{ }^{0} C
$$

em que: I = índice de calor anual da região, calculado com os valores normais de temperaturas mensais;

$\mathrm{i}=\mathrm{n}$. do mês do ano (de 1 a 12 meses),

$\mathrm{Ti}=$ temperatura média mensal.

$$
E T P=E T P \frac{N}{12} \frac{N D}{30}
$$

em que: ETP = evapotranspiração potencial mensal (mm.mês $\mathrm{s}^{-1}$;

$\mathrm{N}=$ fotoperíodo médio mensal (h),

$\mathrm{ND}=$ número de dias do mês. 
A classificação climática de Thornthwaite está baseada em índices: índice efetivo de umidade, índice de eficiência térmica e as variações estacionais de ambos, os quais compõem uma fórmula climática representada por quatro letras consecutivas. A primeira letra da fórmula climática determina o tipo climático e baseia-se no cálculo do índice efetivo de umidade (Im) determinado pelo índice de umidade (Iu) e de aridez (Ia), calculados pelas equaç̃es 5 e 6 ;

$$
\mathrm{I} u=100\left(\frac{E X C}{E T P}\right)
$$

em que: $\mathrm{Iu}=$ índice de umidade;

$\mathrm{EXC}=$ excedente hídrico total $(\mathrm{mm})$,

$\mathrm{ETP}=$ evapotranspiração potencial total $(\mathrm{mm})$.

$$
\mathrm{I} a=100\left(\frac{D E F}{E T P}\right)
$$

em que: Ia= índice de aridez;

$\mathrm{DEF}=$ deficiência hídrica total $(\mathrm{mm})$,

$\mathrm{ETP}=$ evapotranspiração potencial total $(\mathrm{mm})$.

Através dos índices de umidade $\left(\mathbf{I}_{\mathbf{u}}\right)$ e de aridez $\left(\mathbf{I}_{\mathbf{a}}\right)$ foi calculado o índice efetivo de umidade $\left(\mathrm{I}_{\mathrm{m}}\right)$ pela equação 8 ;

$$
\mathrm{I} m=(\mathrm{I} u-0.6 * \mathrm{I} a)
$$

em que: $\operatorname{Im}=$ índice efetivo de umidade;

$$
\begin{aligned}
& \mathrm{I}_{\mathrm{u}}=\text { índice de umidade }(\mathrm{mm}), \\
& \mathrm{I}_{\mathrm{a}}=\text { índice de } \operatorname{aridez}(\mathrm{mm})
\end{aligned}
$$




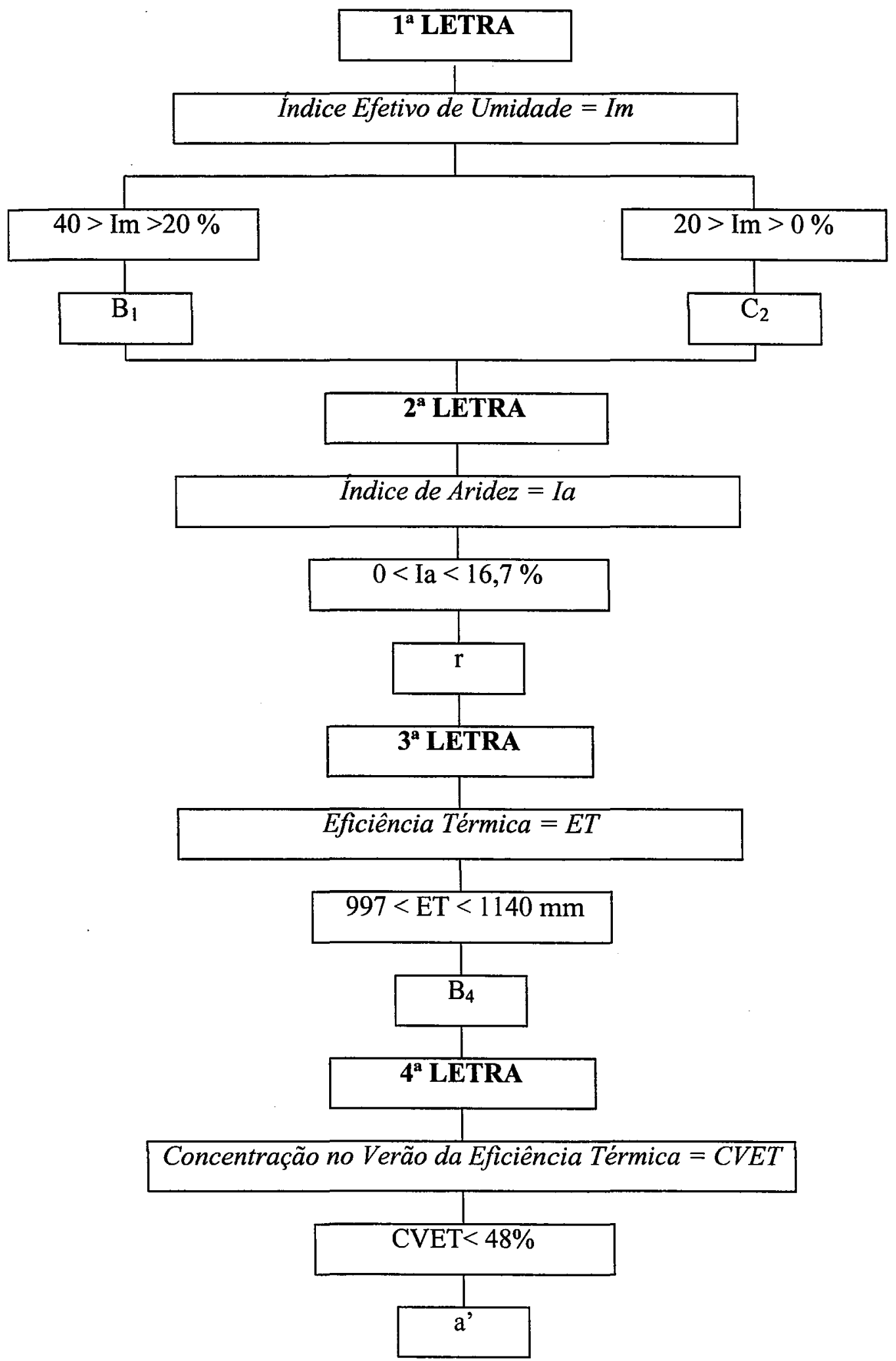

Figura 3: Representação esquemática da seqüência de operações, para obtenção da fórmula climática de acordo com a classificação climática de Thornthwaite, 1948. 
De acordo com estes índices, a classificação climática de Thornthwaite é realizada da seguinte maneira: o índice efetivo de umidade (Im) determina o tipo climático, em que $\mathrm{B}_{1}$ ocorre quando Im encontra-se entre 40 a $20 \%$ e $\mathrm{C}_{2}$ é determinado quando Im está entre 20 a $0 \%$. A segunda letra da fórmula é determinada pelo índice de aridez (Ia), o qual subdivide os tipos climáticos em subtipos. O subtipo $\mathrm{r}$ ocorre quando o índice Ia fica entre 0 a $16,7 \%$, significando pequena ou nenhuma deficiência hídrica. A terceira letra refere-se à eficiência térmica (ET) que é a própria evapotranspiração potencial total (ETP), sendo a evapotranspiração potencial total anual entre 997 a 1140 mm determina o clima mesotérmico $\left(\mathbf{B}_{4}{ }_{4}\right)$ e a quarta letra é determinada pela variação estacional térmica ou concentração de verão da eficiência térmica (CVET), a qual identifica o subtipo a', significando que a estação de crescimento das plantas se concentra no verão abaixo de $48 \%$, calculada pela equação 9 .

$$
C V E T=100\left(\frac{E T P_{\text {verao }}}{E T P_{\text {anual }}}\right)
$$

em que: $\mathrm{CVET}=$ concentração de verão da eficiência térmica

$\mathrm{ETP}_{\text {verão }}=$ soma da evapotranspiração potencial no verão $(\mathrm{Dez}+\mathrm{Jan}+\mathrm{Fev})$, mm.mês ${ }^{-1}$,

$\mathrm{ETP}_{\text {anual }}=$ evapotranspiração total anual (mm.mês $\left.{ }^{-1}\right)$.

Esta classificação climática encontra-se representada na figura 2.

\subsubsection{Balanço hídrico mensal normal, mensal médio e mensal mediano e decendial normal}

Para subsidiar a interpretação dos dados climáticos médios, calculou-se o balanço hídrico mensal normal (BHMN) elaborado com temperaturas e precipitações pluviais médias; o balanço hídrico mensal médio (BHMM), que representa as médias dos componentes dos 80 balanços hídricos seqüenciais mensais; o balanço hídrico 
mensal mediano (BHMMED) realizado com os valores medianos das temperaturas e precipitações pluviais e o balanço hídrico decendial normal (BHDN) elaborado com temperaturas e precipitações médias decendiais. Esses balanços hídricos foram realizados pelo programa computacional balanço hídrico mensal e decendial de Barbieri et al. (1991), baseado em Thornthwaite e Mather (1955) entre o período de 1917-1996, verificando-se ser o mais adequado para análise climática.

\subsubsection{Cálculo das freqüiências e probabilidades dos componentes do balanço hídrico seqüencial (Thornthwaite e Mather, 1955), na escala mensal}

Através dos valores dos componentes do balanço hídrico seqüencial mensal (temperatura do ar, evapotranspiração potencial, precipitação pluvial, armazenamento hídrico, evapotranspiração real, deficiência hídrica e o excedente hídrico) obteve-se a média, valor máximo e valor mínimo, para realização dos cálculos das freqüências e probabilidades, desses componentes através das equações 10,11 e 12 com base em Assis (1996).

Utilizou-se o programa computacional citado no item 3.2.3, com a CAD em três níveis: $50 \mathrm{~mm} ; 100 \mathrm{~mm}$ e $150 \mathrm{~mm}$.

$$
\mathrm{N} C=5[\log (N)]
$$

em que: $\mathrm{NC}=$ número de classes,

$\mathrm{N}=$ número total de dados observados no balanço hídrico mensal.

$$
\mathrm{I} C=\left[V_{\max }-\left(V_{\min }-1\right)\right] / \mathrm{N} C
$$

em que: $\mathrm{IC}=$ intervalo de classe do componente do balanço hídrico;

$\mathrm{V}_{\max }=$ valor máximo do componente do balanço hídrico;

$\mathrm{V}_{\min }=$ valor mínimo do componente do balanço hídrico,

$\mathrm{NC}=$ número de classes.

Após determinar o número de classes (NC) e o intervalo de classes (IC) calculou- 
se a freqüência (F) em cada intervalo de classe para cada componente do balanço hídrico, utilizando a planilha do programa EXCEL.

A partir da freqüência (f) para cada intervalo de classe, calculou-se a probabilidade de ocorrência $(\mathrm{P})$ em porcentagem para cada componente do balanço hídrico (Assis, 1996).

$$
P=\left(F / N^{*} 100\right)
$$

em que: $\mathrm{P}=$ probabilidade de ocorrência para cada intervalo de classe de cada componente do balanço hídrico em porcentagem (\%);

$\mathrm{F}=$ freqüência de ocorrência de eventos de cada componente do balanço hídrico mensal, dentro do intervalo de classe

$\mathrm{N}=$ número total de dados observados do balanço hídrico mensal.

\subsubsection{Cálculo das freqüências e probabilidades dos componentes do balanço hídrico seqüencial (Thornthwaite e Mather -1955) na escala decendial}

Utilizando a mesma metodologia citada no item anterior (3.2.5), os dados decendiais foram organizados na planilha do programa EXCEL, da seguinte forma:

- meses com 31 dias: $\quad 1^{\circ}$ decêndio $=1$ a 10 dias

$2^{\circ}$ decêndio $=11$ a 20 dias

$3^{\circ}$ decêndio $=21$ a 31 dias

- meses com 30 dias: $\quad 1^{\circ}$ decêndio $=1$ a 10 dias

$2^{\circ}$ decêndio $=11$ a 20 dias

$3^{\circ}$ decêndio $=21$ a 30 dias

- meses com 28 e 29 dias: $\quad 1^{0}$ decêndio $=1$ a 10 dias

$2^{\circ}$ decêndio $=11$ a 20 dias

$$
3^{\circ} \text { decêndio }=21 \text { a } 28 \text { ou } 29 \text { dias }
$$

A partir dos valores de cada componente do balanço hídrico, realizaram-se os cálculos das freqüências e probabilidades, conforme procedimento aplicado aos componentes do balanço hídrico seqüencial mensal. 


\section{RESULTADOS E DISCUSSÃO}

\subsection{Classificação climática de Köppen (1918)}

$\mathrm{Na}$ Tabela 1 estão representados os resultados da classificação climática de Köppen, para Piracicaba, utilizando médias móveis nas ordens de $10 ; 15 ; 20 ; 25 ; 30$ e 35 anos, no período de 1917-1996 (80 anos).

Tabela 1- Distribuição das freqüências dos tipos climáticos de Köppen (1918), nas ordens de $10,15,20,25,30$ e 35 anos na área de estudo (Período: 1917-1996).

\begin{tabular}{c|cccccc}
\hline \multirow{2}{*}{$\begin{array}{c}\text { Ordens } \\
\text { (médias móveis }\end{array}$} & \multicolumn{7}{|c}{ Freqüências (n. de ocorrências) } \\
\cline { 2 - 7 } em anos) & \multicolumn{7}{c}{ Tipo climático } & $\begin{array}{c}\text { (C w a) } \\
\text { (C f a) }\end{array}$ & $\begin{array}{c}\text { (C f a } \\
\text { Limite (9 mm) }\end{array}$ & C w a & Limite (10 mm) & A w & $\begin{array}{c}\text { Total } \\
\text { de anos }\end{array}$ \\
\hline $\mathbf{1 0}$ & 34 & 4 & 36 & 5 & 1 & 71 \\
$\mathbf{1 5}$ & 38 & 12 & 28 & 10 & - & 66 \\
$\mathbf{2 0}$ & 31 & 6 & 30 & 13 & - & 61 \\
$\mathbf{2 5}$ & 32 & 7 & 24 & 17 & - & 56 \\
$\mathbf{3 0}$ & 36 & 11 & 15 & 8 & - & 51 \\
$\mathbf{3 5}$ & 31 & 8 & 15 & 10 & - & 46 \\
\hline
\end{tabular}

Identificaram-se dois tipos climáticos $\mathrm{A}$ e $\mathrm{C}$, classificados como pertencentes ao clima tropical chuvoso e três subtipos: Aw, Cwa e Cfa. 
Observou-se que, na ordem de 10 anos, as freqüências foram próximas para o tipo climático Cfa de 34 ocorrências, das quais 4 estavam no limite $(9 \mathrm{~mm})$ para o tipo Cwa. Para o tipo Cwa aconteceram 36 ocorrências, 5 delas no limite $(10 \mathrm{~mm})$ para Cfa e 1 ocorrência para o tipo Aw, mostrando que os tipos climáticos se confundem nesta ordem. Para a ordem de 15 anos o tipo climático predominante foi o Cfa apresentando 38 ocorrências, sendo 12 no limite para Cwa e 28 ocorrências oara o tipo Cwa, em que 10 foram no limite para o Cfa. Embora a freqüência do limite para o tipo Cwa tenha sido 12 , sendo maior em relação ao Cfa que foi 10 , conelui-se que o clima pode ser considerado Cfa.

$\mathrm{Na}$ ordem de 20 anos, as freqüências entre os tipos climáticos foram de 31 ocorrências para o $\mathrm{Cfa}$, das quais 6 ocorreram no limite para Cwa e 30 ocorrências para Cwa, sendo 13 casos no limite para $\mathrm{Cfa}$, com as freqüências apresentando-se próximas para os dois tipos climáticos. Contudo, os casos no limite para o Cfa foram superiores, o que indica, para esta ordem, o tipo climático Cfa. Para a ordem de 25 anos, a freqüência foi de 32 ocorrências para o Cfa, 7 delas no limite para Cwa e das 24 ocorrências para o Cwa, 17 delas ocorreram no limite para Cfa, sugerindo, desta forma, que nessa ordem o clima pode ser considerado Cfa. Na ordem de 30 e 35 anos as freqüências foram de 36 e 31 ocorrências para o tipo Cfa, sendo 11 e 8 delas no limite para Cwa e dos 15 casos para o Cwa, 8 e 10 ocorreram no limite para Cfa. Portanto, o tipo climático predominante, para estas duas ordens foi Cfa.

Os dados acima evidenciam que, para todas as ordens o tipo climático enquadrouse no tipo Cfa, exceto para a ordem de 10 anos. Isso indica que para a análise climática, utilizando-se a classificação climática de Köppen, pode-se considerar que 35 anos de observação ainda não seriam suficientes para diferenciar o tipo de clima entre Cfa e Cwa na região de Piracicaba, tendo em vista que para a ordem de 35 anos, em 46 casos observados, 31 foram do tipo Cfa e 15 do tipo Cwa.

Salienta-se que, sendo os climas Cfa e Cwa muito próximos quanto à caracterização, uma vez que o tipo Cfa ocorre quando a precipitação do mês mais úmido é menor que 10 vezes do mês mais seco, e Cwa quando a precipitação do mês mais úmido é igual ou maior que 10 vezes do mês mais seco, dessa forma, com apenas 10 anos 
de observação o tipo climático de Piracicaba seria identificado satisfatoriamente como Cwa e Cfa.

Observa-se, conforme citado por Ayoade (1986) que a Organização Meteorológica Mundial (OMM) considera como 30 anos o mínimo de observação para uma análise climática. Esta recomendação, portanto, não é adequada para Piracicaba conforme o exposto no parágrafo anterior.

A Tabela 2, a seguir, mostra os resultados da classificação climática de Köppen, em probabilidades.

Tabela 2- Distribuição das probabilidades (\%) dos tipos climáticos de Köppen (1918), nas ordens de 10, 15, 20, 25, 30 e 35 anos na área de estudo (Período: 1917-1996).

\begin{tabular}{|c|c|c|c|c|c|c|}
\hline \multirow{3}{*}{$\begin{array}{l}\text { Ordens } \\
\text { (médias móveis } \\
\text { em anos) }\end{array}$} & \multicolumn{6}{|c|}{ Probabilidades (\%) } \\
\hline & \multicolumn{5}{|c|}{ Tipo climático } & \multirow[b]{2}{*}{$\begin{array}{l}\text { Total } \\
\text { de anos }\end{array}$} \\
\hline & Cfa & $\begin{array}{c}\text { (C f a) } \\
\text { Limite }(9 \mathrm{~mm})\end{array}$ & Cw a & $\begin{array}{c}\text { (C w a) } \\
\text { Limite (10 mm) }\end{array}$ & $\mathbf{A} \mathbf{w}$ & \\
\hline 10 & 48 & 6 & 51 & 7 & 1 & 71 \\
\hline 15 & 58 & 18 & 42 & 15 & - & 66 \\
\hline 20 & 51 & 10 & 49 & 21 & - & 61 \\
\hline 25 & 57 & 13 & 43 & 30 & - & 56 \\
\hline 30 & 71 & 22 & 29 & 16 & - & 51 \\
\hline 35 & 67 & 17 & 33 & 22 & - & 46 \\
\hline
\end{tabular}

Verificou-se que, na ordem de 10 anos existe $48 \%$ de probabilidade de ocorrência do tipo climático $\mathrm{Cfa}$, sendo que $6 \%$ ocorreu no limite para o $\mathrm{Cwa}$; dos $51 \%$ do tipo Cwa, $7 \%$ encontra-se no limite para o tipo Cfa e $1 \%$ para o tipo Aw, demostrando que, nessa ordem os tipos climáticos se confundem. Na ordem de 15 anos, o tipo Cfa apresentou $58 \%$ de probabilidade de ocorrência, sendo que $18 \%$ encontra-se no limite para o Cwa; dos $42 \%$ apresentados para o tipo Cwa, $15 \%$ ocorreu no limite para Cfa, indicando para esta ordem o tipo climático Cfa. Para a ordem de 20 anos, 51\% de ocorrência foi para o tipo $\mathrm{Cfa}, 10 \%$ aconteceu no limite para o Cwa e dos $49 \%$ para o tipo Cwa, 21\% encontra-se no limite para o Cfa. Dessa forma, embora as probabilidades dos tipos climáticos tenham sido próximas, a indicação é para o tipo Cfa, pois o número 
de ocorrências no limite para o Cfa foi maior (21\%).

Para a ordem de 25 anos, verificou-se $57 \%$ de probabilidade para o tipo Cfa, sendo $13 \%$ no limite para Cwa e dos $43 \%$ de probabilidades para o tipo Cwa, $30 \%$ ocorreu no limite para o $\mathrm{Cfa}$, evidenciando o tipo climático $\mathrm{Cfa}$. Na ordem de 30 anos, ocorreu $71 \%$ para o tipo Cfa, $22 \%$ aparece no limite para o Cwa e dos $29 \%$ de probabilidade para o tipo Cwa, 16\% ocorreu no limite para o Cfa. Para a ordem de 35 anos, aparece $67 \%$ para o tipo Cfa, com $17 \%$ no limite para o Cwa e dos $33 \%$ de ocorrências para o tipo Cwa, 22\% ocorreu no limite para o Cfa, mostrando o tipo Cfa para as duas ordens.

Dessa maneira, somente para a ordem de 10 anos, as freqüencias e as probabilidades se confundem entre os dois tipos climáticos: Cfa e Cwa; nas demais ordens, o clima enquadrou-se no tipo Cfa. Também constatou-se que o tipo climático Aw ocorreu no período de 1929 a 1938 sendo que a temperatura média foi de $18,0^{\circ} \mathrm{C}$ somente na ordem de 10 anos, resuitado que coincide segundo Ometto (1991), com a tendência crescente de aumento da temperatura média nesse período.

Analisando-se somente os efeitos dos últimos 10 anos nestas tendências, na tentativa de observar mudanças climáticas na atualidade, outro aspecto importante observado foi que, a partir da média móvel de 15 anos (1958 a 1972); 20 anos (1953 a 1972); 25 anos (1948 a 1972); 30 anos (1943 a 1972) e 35 anos (1938 a 1972) até o ano de 1996, o tipo climático da região passou do tipo Cwa para o tipo Cfa. Isto se deve ao aumento da precipitação pluvial na estação seca (inverno) maior que $26 \mathrm{~mm}$ e uma diminuição na estação chuvosa (verão) menor de $233 \mathrm{~mm}$, conforme representado nos quadros de 1 a 6 do Anexo A e quadro 1 a 6 do Anexo B. Estes resultados sugerem uma tendência de decréscimo das chuvas, principalmente, nos meses de janeiro e dezembro de $19,3 \mathrm{~mm}$ e $36,0 \mathrm{~mm}$, respectivamente, conforme mostrado por Galvani (1997).

Analisando as 15 últimas observações de cada ordem (médias móveis), constatouse que somente na ordem de 10 anos (1973 a 1996) ocorreu o tipo Cwa (5 ocorrências); para as demais ordens, o tipo predominante foi Cfa. Para a última observação de cada ordem, o tipo climático foi Cfa, exceto para a ordem de 10 anos entre os anos de 1987 a 1996 com predomínio do tipo Cwa, mostrando que existe uma certa tendência dos meses 
úmidos (DEZ, JAN e FEV) se tornarem um pouco menos úmidos e os meses mais secos (JUN, JUL e AGO) se tornarem menos secos.

\subsection{Classificação climática de Thornthwaite (1948)}

Os resultados da classificação climática de Thornthwaite (1948) estão representados na Tabela 3 .

Tabela 3- Distribuição das freqüências dos tipos climáticos de Thornthwaite (1948), nas ordens de 10, 15, 20, 25, 30 e 35 anos na área de estudo (Período: 1917-1996).

\begin{tabular}{|c|c|c|c|c|c|}
\hline \multirow{4}{*}{$\begin{array}{l}\text { Ordens } \\
\text { (médias móveis } \\
\text { em anos) }\end{array}$} & \multicolumn{5}{|c|}{ Freqüências (n. de ocorrências) } \\
\hline & \multicolumn{5}{|c|}{ Tipo climático } \\
\hline & & $\mathbf{B}_{1} \mathbf{r} \mathbf{B}_{4}^{\prime} \mathbf{a}^{\prime}$ & & $C_{2} \times B_{4}^{\prime} a^{\prime}$ & Total \\
\hline & $\mathbf{B}_{1} \mathbf{r} \mathbf{B}_{4}^{\prime} \mathbf{a}^{\prime}$ & Limite $(21 \%)$ & $\mathrm{C}_{2} \times \mathrm{B}_{4}^{\prime} \mathbf{a}^{\prime}$ & Limite (20\%) & de anos \\
\hline 10 & 50 & 4 & 21 & 5 & 71 \\
\hline 15 & 52 & 3 & 14 & 7 & 66 \\
\hline 20 & 47 & 4 & 14 & 7 & 61 \\
\hline 25 & 46 & 3 & 10 & 6 & 56 \\
\hline 30 & 47 & 4 & 4 & 2 & 51 \\
\hline 35 & 43 & 3 & 3 & 3 & 46 \\
\hline
\end{tabular}

Através dessa classificação climática constatou-se que a região de Piracicaba apresentou dois tipos climáticos: $B_{1}$ e $C_{2}$. $O$ tipo $B_{1}$ caracteriza-se pelo tipo úmido (limite de 20 a $40 \%$ ) e o $\mathrm{C}_{2}$ pelo tipo úmido e subúmido (entre 20 e $0 \%$ ). Os subtipos foram: $\mathrm{B}_{1} \mathrm{r}$ $\mathrm{B}^{\prime}{ }_{4} \mathrm{a}^{\prime}$ e $\mathrm{C}_{2} \mathrm{r} \mathrm{B}^{\prime}{ }_{4} \mathrm{a}^{\prime}$, os quais são climas úmidos, com pequena ou nenhuma deficiência de água, mesotérmicos, sendo que $48 \%$ da estação de crescimento das plantas se concentra no verão (Ayoade, 1986).

Para a ordem de 10 anos, a freqüência de ocorrência do tipo climático $\mathrm{B}_{1} \mathrm{r} \mathrm{B}_{4}{ }_{4} \mathrm{a}^{\prime}$ foi igual a 50 vezes, das quais 4 foram no limite (21\%) para o tipo $\mathrm{C}_{2} \mathrm{r} \mathrm{B}^{\prime}{ }_{4} \mathrm{a}^{\prime}$ e das 21 ocorrências para o tipo $\mathrm{C}_{2} \mathrm{r} \mathrm{B}_{4}{ }_{4} \mathrm{a}^{\prime}, 5$ aconteceram no limite $(20 \%)$ para o $\mathrm{B}_{1} \mathrm{r} \mathrm{B}_{4}{ }_{4} \mathrm{a}^{\prime}$, definindo-se para esta ordem o tipo $\mathrm{B}_{1} \mathrm{r}_{\mathrm{B}^{\prime}}{ }_{4} \mathrm{a}^{\prime}$. Para as ordens de 15 e 20 anos identificaram-se 52 e 47 ocorrências para o tipo $\mathrm{B}_{1} \mathrm{r} \mathrm{B}_{4}^{\prime} \mathrm{a}^{\prime}$, sendo 3 e 4 ocorrências no 
limite para $\mathrm{C}_{2} \mathrm{rB}_{4} \mathrm{a}^{\prime}$ e das 14 ocorrências do tipo $\mathrm{C}_{2} \mathrm{r} \mathrm{B}_{4}{ }_{4} \mathrm{a}^{\prime}$ para ambas ordens, 7 delas foram no limite do $\mathrm{C}_{2} \mathrm{rB}_{4}{ }_{4} \mathrm{a}^{\prime}$, isto mostra o predomínio para o tipo $\mathrm{B}_{1} \mathrm{r} \mathrm{B}_{4} \mathrm{a}^{\prime}$.

Para a ordem de 25 anos, ocorreram 46 casos para o $\mathrm{B}_{1} \mathrm{r} \mathrm{B}_{4}{ }_{4} \mathrm{a}^{\prime}$, sendo que 3 casos foram no limite para o $\mathrm{C}_{2} \mathrm{r} \mathrm{B}_{4} \mathrm{a}^{\prime}$, das 10 ocorrências para o tipo $\mathrm{C}_{2} \mathrm{r} \mathrm{B}_{4} \mathrm{a}^{\prime} 6$ ocorrências ficaram no limite para $\mathrm{B}_{1} \mathrm{r} \mathrm{B}^{\prime}{ }_{4} \mathrm{a}^{\prime}$, podendo considerar o tipo $\mathrm{B}_{1} \mathrm{r} \mathrm{B}_{4}{ }_{4} \mathrm{a}^{\prime}$. Na ordem de 30 e 35 anos, as freqüencias do tipo $B_{1}+B_{4}^{\prime} a^{\prime}$ apresentaram 47 e 43 ocorrências, sendo que 4 e 3 estiveram no limite para $\mathrm{C}_{2} \mathrm{r} \mathrm{B}^{\prime}{ }_{4} \mathrm{a}^{\prime}$, respectivamente, e das 4 e 3 ocorrências para o tipo $\mathrm{C}_{2} \mathrm{r} \mathrm{B}_{4}{ }_{4} \mathrm{a}^{\prime} 2$ e 3 foram no limite, sendo predominante o tipo climático $\mathrm{B}_{1} \mathrm{r} \mathrm{B}_{4}{ }_{4} \mathrm{a}^{\prime}$. Assim, para todas as ordens, o tipo climático predominante foi $\mathrm{B}_{1} \mathrm{r}$ $\mathrm{B}^{\prime}{ }_{4} \mathrm{a}^{\prime}$, úmido.

Na Tabela 4 abaixo, estão representados os valores em probabilidades da classificação climática de Thornthwaite.

Tabela 4- Distribuição das probabilidades (\%) dos tipos climáticos de Thornthwaite (1948), nas ordens de 10, 15, 20, 25, 30 e 35 anos (Período: 1917-1996).

\begin{tabular}{|c|c|c|c|c|c|}
\hline \multirow{4}{*}{$\begin{array}{c}\text { Ordens } \\
\text { (médias móveis } \\
\text { em anos) }\end{array}$} & \multicolumn{5}{|c|}{ Probabilidades (\%) } \\
\hline & \multicolumn{5}{|c|}{ Tipo climático } \\
\hline & & $B_{1} \times B_{4}^{\prime} a^{\prime}$ & & $C_{2} r B^{\prime} a^{\prime}$ & Total \\
\hline & $\mathbf{B}_{1} \mathbf{r} \mathbf{B}_{4}{ }_{4} \mathbf{a}^{\prime}$ & Limite $(21 \%)$ & $\mathrm{C}_{2} \times \mathrm{B}_{4}^{\prime} \mathrm{a}^{\prime}$ & Limite (20\%) & de anos \\
\hline 10 & 70 & 6 & 30 & 7 & 71 \\
\hline 15 & 79 & 5 & 21 & 11 & 66 \\
\hline 20 & 77 & 7 & 23 & 11 & 61 \\
\hline 25 & 82 & 5 & 18 & 11 & 56 \\
\hline 30 & 92 & 8 & 8 & 4 & 51 \\
\hline 35 & 93 & 7 & 7 & 7 & 46 \\
\hline
\end{tabular}

Verificou-se que, para a ordem de 10 anos, registrou $70 \%$ de probabilidade para o tipo climático $\mathrm{B}_{1} \mathrm{rB} \mathrm{B}_{4}{ }_{4} \mathrm{a}^{\prime}, 6 \%$ ficaram no limite para $\mathrm{C}_{2} \mathrm{r} \mathrm{B}_{4}{ }_{4} \mathrm{a}^{\prime}, 30 \%$ para o tipo $\mathrm{C}_{2} \mathrm{r} \mathrm{B}_{4}{ }_{4}$ $a^{\prime}, 7 \%$ ocorreu no limite para $\mathrm{B}_{1} \mathrm{rB}^{\prime}{ }_{4} \mathrm{a}^{\prime}$, predominando o tipo $\mathrm{B}_{1} \mathrm{rB}^{\prime}{ }_{4} \mathrm{a}^{\prime}$. Para as ordens de 15 e 20 anos, registrou-se $79 \%$ e $77 \%$ de probabilidade para o tipo $\mathrm{B}_{1} \mathrm{r} \mathrm{B}^{\prime}{ }_{4} \mathrm{a}^{\prime}$, sendo que $5 \%$ e $7 \%$ aconteceram no limite para $\mathrm{C}_{2} \mathrm{r} \mathrm{B}^{\prime}{ }_{4}$ a' e $21 \%$ e $23 \%$ para o tipo $\mathrm{C}_{2} \mathrm{r} \mathrm{B}_{4}{ }_{4} \mathrm{a}^{\prime}$, sendo $11 \%$ de ocorrências no limite. $\mathrm{O}$ tipo climático predominante foi $\mathrm{B}_{1} \mathrm{rB}_{4} \mathrm{a}^{\prime}$. 
A ordem de 25 anos apresentou $82 \%$ de probabilidade de ocorrência do tipo $\mathrm{B}_{1} \mathrm{r}$ $\mathrm{B}^{\prime}{ }_{4} \mathrm{a}^{\prime}$ e $18 \%$ para o tipo $\mathrm{C}_{2} \mathrm{r} \mathrm{B}^{\prime}{ }_{4} \mathrm{a}^{\prime}$, sendo $5 \%$ no limite para o $\mathrm{C}_{2} \mathrm{r} \mathrm{B}^{\prime}{ }_{4} \mathrm{a}^{\prime}$ e $11 \%$ no limite $B_{1} r B^{\prime}{ }_{4} a^{\prime}$ respectivamente, predominando, desta forma, o tipo $B_{1} r B^{\prime}{ }_{4} a^{\prime}$. Nas ordens de 30 e 35 anos a probabilidade de ocorrência foi de $92 \%$ e $93 \%$ para o tipo $\mathrm{B}_{1} \mathrm{~T}$ $\mathrm{B}_{4}{ }_{4} \mathrm{a}^{\prime}$, sendo que $7 \%$ foram no limite para $\mathrm{C}_{2} \mathrm{rB}^{\prime}{ }_{4} \mathrm{a}^{\prime}$. Dos $7 \%$ de ocorrências para o $\mathrm{C}_{2} \mathrm{r}$ $\mathrm{B}_{4}{ }_{4} \mathrm{a}^{\prime}$, estes apresentaram-se no limite. Dessa forma identificaram-se, nestas duas ordens, o predomínio do tipo $\mathrm{B}_{1} \mathrm{r}_{\mathrm{B}^{\prime}}{ }_{4} \mathrm{a}^{\prime}$. Assim, constatou-se para todas as ordens a predominância de ocorrência deste tipo climático.

As 15 últimas observações de cada ordem (médias móveis), mostraram que o tipo climático $\mathrm{C}_{2} \mathrm{r} \mathrm{B}_{4}{ }_{4} \mathrm{a}^{\prime}$ apresentou 1 ocorrência para as ordens de 10 e 30 anos e 2 ocorrências para as ordens 20,25 e 35 anos, as quais ocorreram no limite (20\%), evidenciando o tipo climático $\mathrm{B}_{1} \times \mathrm{B}^{\prime}{ }_{4} \mathrm{a}^{\prime}$. Para a última observação, o predomínio foi do tipo climático $\mathrm{B}_{1}+\mathrm{B}^{\prime}{ }_{4} \mathrm{a}^{\prime}$.

Dessa maneira, identificou-se que a tipologia climática de Thornthwaite apresentou mais estabilidade em relação à de Köppen, no entanto, as duas tipologias climáticas mostraram que, para a ordem de 30 anos, o clima de Piracicaba é úmido $\left(B_{1} r\right.$ $\mathrm{B}^{\prime}{ }_{4}$ a' e Cfa), respectivamente (resultado da classificação de Köppen que difere de Camargo (1978)). Isto indica uma tendência de aumento de precipitações pluviais no inverno como já foi dito antes. Para esta análise, a probabilidade de classificar essa região como sendo do tipo $\mathrm{B}_{1} \mathrm{rB}_{4}{ }_{4} \mathrm{a}^{\prime}$, com 30 anos de observação, é de $93 \%$ e com 10 anos de observação é de $70 \%$. Para essa região, apenas 10 anos de observação meteorológica poderịa ser satisfatório para sua classificação, uma vez que os tipos $B_{1}$ e $C_{2}$ diferenciamse por apenas $1 \mathrm{~mm}$, pois enquanto $\mathrm{B}_{1}$ caracteriza-se pelo Índice efetivo de umidade (Im) entre 40 a $20 \%$ no tipo $\mathrm{C}_{2}$ o Im fica entre 20 e $0 \%$. 
4.3 Balanço hídrico mensal normal (BHMN), mensal médio (BHMME), mensal mediano (BHMMED) e decendial normal (BHDN) e suas classificações climáticas entre o período de 1917 a 1996 da área de estudo.

Analisando o balanço hídrico mensal mensal - BHMN (1917-1996) e o balanço hídrico normal decendial - BHDN (1917-1996), para a CAD: $100 \mathrm{~mm}$, não foram observadas grandes diferenças entre os componentes dos balanços hídricos normais mensais (Tabela 5) e normais decendiais (Tabela 6), pois, em relação ao tipo climático, enquadram-se na tipologia climática Cfa de Köppen e no tipo $\mathrm{B}_{1} \mathrm{r} \mathrm{B}_{4}{ }_{4} \mathrm{a}^{\prime}$ de Thornthwaite.

Tabela 5- Balanço Hídrico Mensal Normal - BHMN (1917-1996) de Piracicaba, segundo Thornthwaite e Mather - 1955 (CAD: $100 \mathrm{~mm}$ )

\begin{tabular}{|c|c|c|c|c|c|c|c|}
\hline \multicolumn{8}{|c|}{ Componentes do Balance Hídrico } \\
\hline Meses & $T\left(C^{\circ} \mathrm{C}\right)$ & $\operatorname{ETP}(\mathrm{mm})$ & $P(\mathrm{~mm})$ & $\operatorname{ARM}(\mathbf{m m})$ & $\operatorname{ETR}(\mathbf{m m})$ & DEF $(\mathrm{mm})$ & EXC (mm) \\
\hline 1 & 24,4 & 126 & 227 & 100 & 126 & 0 & 100 \\
\hline 2 & 24,6 & 112 & 189 & 100 & 112 & 0 & 76 \\
\hline 3 & 24,0 & 111 & 143 & 100 & 111 & 0 & 31 \\
\hline 4 & 21,9 & 81 & 66 & 85 & 80 & 1 & 0 \\
\hline 5 & 19,2 & 57 & 52 & 80 & 56 & $\mathbf{0}$ & 0 \\
\hline 6 & 17,6 & 44 & 42 & 79 & 43 & 0 & 0 \\
\hline 7 & 17,3 & 44 & 25 & 65 & 38 & 5 & 0 \\
\hline 8 & 19,2 & 59 & 29 & 47 & 46 & 13 & 0 \\
\hline 9 & 20,7 & 73 & 63 & 43 & 67 & 5 & 0 \\
\hline 10 & 22,2 & 94 & 110 & 58 & 94 & 0 & 0 \\
\hline 11 & 23,2 & 106 & 131 & 82 & 106 & 0 & 0 \\
\hline 12 & 23.9 & 121 & 202 & 100 & 121 & 0 & 63 \\
\hline Total & 21.0 & 1033 & 1279 & & 1006 & 27 & 272 \\
\hline
\end{tabular}


Tabela 6- Balanço Hídrico Decendial Normal - BHND (1917-1996) de Piracicaba, segundo Thornthwaite e Mather - 1955 (CAD: $100 \mathrm{~mm}$ )

Componentes do Balanco Hídrico

\begin{tabular}{|c|c|c|c|c|c|c|c|}
\hline Decêndio & $\mathrm{T}\left({ }^{\circ} \mathrm{C}\right)$ & ETP (mm) & $P(\mathbf{m m})$ & $\operatorname{ARM}(\mathbf{m m})$ & $\operatorname{ETR}(\mathrm{mm})$ & DEF (mm) & EXC $(\mathbf{m m})$ \\
\hline 1 & 24,3 & 40 & 72 & 100 & 40 & 0 & 31 \\
\hline 2 & 24,3 & 40 & 82 & 100 & 40 & 0 & 42 \\
\hline 3 & 24,4 & 44 & 73 & 100 & 44 & 0 & 28 \\
\hline 4 & 24,4 & 39 & 76 & 100 & 39 & 0 & 37 \\
\hline 5 & 24,7 & 40 & 61 & 100 & 40 & 0 & 21 \\
\hline 6 & 24,5 & 31 & 51 & 100 & 31 & 0 & 20 \\
\hline 7 & 24,4 & 38 & 52 & 100 & 38 & 0 & 14 \\
\hline 8 & 24,1 & 36 & 48 & 100 & 36 & 0 & 11 \\
\hline 9 & 23,6 & 37 & 42 & 100 & 37 & 0 & 5 \\
\hline 10 & 22,7 & 30 & 27 & 96 & 30 & 0 & $\mathbf{0}$ \\
\hline 11 & 21,8 & 26 & 22 & 92 & 26 & 0 & $\mathbf{0}$ \\
\hline 12 & 21,1 & 24 & 16 & 85 & 23 & 0 & 0 \\
\hline 13 & 20,0 & 21 & 17 & 82 & 20 & 0 & 0 \\
\hline 14 & 19,3 & 18 & 18 & 82 & 18 & 0 & 0 \\
\hline 15 & 18,3 & 18 & 15 & 80 & 17 & 0 & 0 \\
\hline 16 & 17,6 & 14 & 15 & 80 & 14 & 0 & 0 \\
\hline 17 & 17,6 & 14 & 15 & 81 & 14 & 0 & 0 \\
\hline 18 & 17,6 & 14 & 10 & 78 & 14 & 0 & 0 \\
\hline 19 & 17,3 & 14 & 8 & 74 & 12 & 1 & 0 \\
\hline 20 & 17,1 & 13 & 9 & 71 & 12 & 1 & 0 \\
\hline 21 & 17,6 & 16 & 7 & 64 & 13 & 3 & 0 \\
\hline 22 & 18,6 & 17 & 7 & 58 & 13 & 3 & 0 \\
\hline 23 & 19,1 & 19 & 7 & 52 & 13 & 5 & 0 \\
\hline 24 & 19,7 & 22 & 15 & 48 & 19 & 3 & 0 \\
\hline 25 & 20,1 & 22 & 17 & 45 & 19 & 2 & 0 \\
\hline 26 & 20,7 & 24 & 23 & 45 & 23 & 0 & 0 \\
\hline 27 & 21,2 & 26 & 22 & 43 & 24 & 2 & 0 \\
\hline 28 & 22,1 & 29 & 31 & 45 & 29 & 0 & 0 \\
\hline 29 & 22,0 & 29 & 39 & 55 & 29 & 0 & 0 \\
\hline 30 & 22,5 & 35 & 38 & 58 & 35 & 0 & 0 \\
\hline 31 & 23,0 & 34 & 36 & 60 & 34 & 0 & 0 \\
\hline 32 & 23,2 & 35 & 43 & 68 & 35 & 0 & 0 \\
\hline 33 & 23,2 & 35 & 50 & 83 & 35 & 0 & 0 \\
\hline 34 & 23,8 & 38 & 58 & 100 & 38 & 0 & 2 \\
\hline 35 & 23,9 & 39 & 69 & 100 & 39 & 0 & 30 \\
\hline 36 & 23,8 & 42 & 73 & 100 & 42 & 0 & 30 \\
\hline Total & 21 & 1031 & 1281 & & 1004 & 26 & 276 \\
\hline
\end{tabular}


Comparando-se o BHMN com o BHDN observa-se que os resultados são praticamente idênticos, ou seja, se forem divididos os valores dos componentes mensais por 3, encontram-se os valores do BHDN. Como exemplo: a ETP mensal de janeiro apresentou $126 \mathrm{~mm}$. Dividindo-se por 3, obtém-se $42 \mathrm{~mm}$. Tal valor é muito próximo do encontrado nos 3 decêndios do BHDN, concluindo-se que, para a análise climática, é desnecessária a elaboração do BHDN.

Em relação ao balanço hídrico mensal médio - BHMME (Tabela 7) e o balanço hídrico mensal mediano - BHMMED (Tabela 8) verificaram-se grandes diferenças. No balanço hídrico médio, a temperatura, a evapotranspiração potencial e a precipitação pluvial foi $22,0^{\circ} \mathrm{C}, 1046 \mathrm{~mm}$ e $1269 \mathrm{~mm}$, respectivamente. Para o balanço hídrico mediano, os resultados foram $21,0^{\circ} \mathrm{C}, 1024 \mathrm{~mm}$ e $1170 \mathrm{~mm}$, identificando maiores valores nos totais pluviométricos. Já a evapotranspiração real foi de $921 \mathrm{~mm}$ no balanço hídrico médio e $954 \mathrm{~mm}$ para o mediano; a deficiência hídrica foi menor no balanço hídrico mediano (66 mm) em relação ao médio de $125 \mathrm{~mm}$, e o excedente hídrico apresentou $348 \mathrm{~mm}$ no balanço médio e $207 \mathrm{~mm}$ para o mediano, mostrando menores valores no excedente hídrico.

A classificação climática pelo método de Thornthwaite é também recomendável, tanto para o BHNM, BHNME como para o BHMMED, pois os três balanços, na região de Piracicaba, enquadraram no tipo climático $\mathrm{B}_{1} \mathrm{r} \mathrm{B}^{\prime}{ }_{4} \mathrm{a}^{\prime}$. Já pelo método de Köppen, o BHNM e o BHMME apresentaram o mesmo tipo climático Cfa, considerando-se estes os mais adequados, pois o BHMMED identificou-se como $\mathrm{Cwa}$, evidenciando-se que as chuvas não têm uma distribuição normal. Do ponto de vista estatístico, evidencia-se que os valores medianos são os mais adequados para análise geral do clima. 
Tabela 7- Balanço Hídrico Mensal Médio - BHMME (1917-1996) de Piracicaba, segundo Thornthwaite e Mather - 1955 (CAD: $100 \mathrm{~mm}$ )

\begin{tabular}{cccccccc}
\hline \multicolumn{7}{c}{ Componentes do Balanco Hidrico Médio } \\
\hline Meses & T $\mathbf{0} \mathbf{C}$ ) & ETP $(\mathbf{m m})$ & P (mm) & ARM (mm) & ER (mm) & DEF (mm) & EXC(mm) \\
\hline 1 & 24,4 & 127 & 222 & 92 & 124 & 3 & 95 \\
2 & 24,6 & 112 & 185 & 88 & 107 & 5 & 81 \\
3 & 24,0 & 112 & 143 & 83 & 106 & 6 & 41 \\
4 & 21,8 & 82 & 65 & 66 & 71 & 11 & 11 \\
5 & 19,1 & 59 & 52 & 61 & 48 & 11 & 9 \\
6 & 17,6 & 46 & 43 & 59 & 37 & 9 & 9 \\
7 & 17,3 & 47 & 27 & 50 & 33 & 14 & 3 \\
8 & 19,1 & 61 & 30 & 40 & 39 & 22 & 1 \\
9 & 20,7 & 75 & 63 & 42 & 53 & 22 & $\mathbf{8}$ \\
10 & 22,2 & 97 & 111 & 56 & 86 & 11 & 12 \\
11 & 23,1 & 107 & 130 & 68 & 99 & $\mathbf{8}$ & 18 \\
12 & 23,8 & 122 & 199 & 89 & 119 & 3 & 60 \\
\hline Total & $\mathbf{2 2 . 0}$ & $\mathbf{1 0 4 6}$ & $\mathbf{1 2 6 9}$ & $\mathbf{7 9 5}$ & $\mathbf{9 2 1}$ & $\mathbf{1 2 5}$ & $\mathbf{3 4 8}$ \\
\hline
\end{tabular}

Tabela 8- Balanço Hídrico Mensal Mediano - BHMMED (1917-1996) de Piracicaba, segundo Thornthwaite e Mather - 1955 (CAD: $100 \mathrm{~mm}$ )

\begin{tabular}{|c|c|c|c|c|c|c|c|}
\hline \multicolumn{8}{|c|}{ Componentes do Balanco Hídrico } \\
\hline Meses & $\mathrm{T}\left(\mathbf{C}^{\circ} \mathrm{C}\right)$ & $\operatorname{ETP}(\mathbf{m m})$ & $P(\mathbf{m m})$ & ARM (mm) & $\operatorname{ER}(\mathrm{mm})$ & DEF (mm) & EXC $(\mathbf{m m})$ \\
\hline 1 & 24,4 & 126 & 220 & 100 & 126 & 0 & 93 \\
\hline 2 & 24,6 & 112 & 177 & 100 & 112 & 0 & 64 \\
\hline 3 & 24,0 & 111 & 140 & 100 & 111 & 0 & 28 \\
\hline 4 & 21,9 & 81 & 53 & 75 & 77 & 3 & 0 \\
\hline 5 & 19,1 & 57 & 42 & 64 & 52 & 4 & 0 \\
\hline 6 & 17,6 & 44 & 32 & 56 & 39 & 4 & 0 \\
\hline 7 & 17,3 & 44 & 15 & 42 & 29 & 15 & 0 \\
\hline 8 & 19,1 & 59 & 20 & 28 & 33 & 25 & 0 \\
\hline 9 & 20,7 & 73 & 53 & 23 & 58 & 15 & 0 \\
\hline 10 & 22,1 & 93 & 101 & 30 & 93 & 0 & 0 \\
\hline 11 & 23,0 & 104 & 124 & 49 & 104 & 0 & 0 \\
\hline 12 & 23.8 & 120 & 193 & 100 & 120 & 0 & 22 \\
\hline Total & 21.5 & 1024 & 1170 & 767 & 954 & 66 & 207 \\
\hline
\end{tabular}




\subsection{Variabilidade temporal dos totais anuais pluviométricos e temperaturas médias anuais}

A Figura 4 apresenta os totais de precipitação pluvial de Piracicaba. Verificou-se que a média de precipitação do período foi de $1269 \mathrm{~mm}$, sendo o ano de 1921 menos chuvoso $(812 \mathrm{~mm})$ e 1983 o mais chuvoso $(2018 \mathrm{~mm})$. Pode-se observar, também, que os anos de 1931, 1943, 1982, 1995, 1996 foram anos muitos chuvosos. Salienta-se que, a partir da década de 80 , esses episódios acentuaram-se, ocorrendo três vezes. Os anos menos chuvosos foram 1924, 1956, 1963, 1978, 1984.

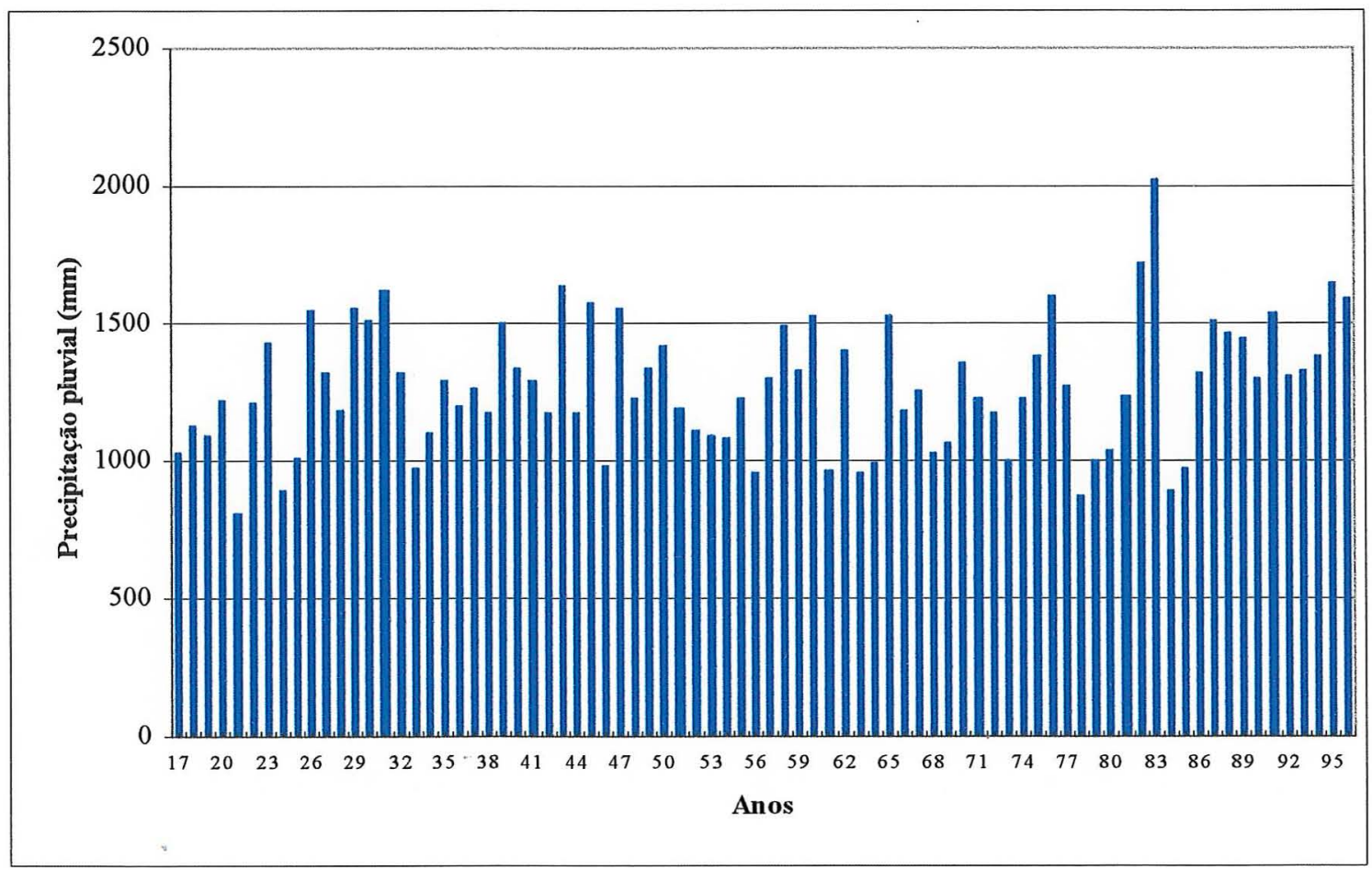

Figura 4- Totais pluviométricos anuais da área de estudo. Período: 1917-1996

Pela Figura 5, evidencia-se que, a média da temperatura anual do período foi $21,5^{\circ} \mathrm{C}$ e a maior temperatura registrada ocorreu no ano $1936\left(23,1^{\circ} \mathrm{C}\right)$, seguido pelos anos de 1994, 1984, 1963 e 1940; a menor temperatura média $\left(19,7^{\circ} \mathrm{C}\right)$ aconteceu em 1917 e, posteriormente, nos anos de 1980, 1976 e 1972. Outro fato importante ocorrido 
foi que a partir da década de 80 foram registrados dois episódios de temperatura média anual elevada $\left(1984=22,7^{\circ} \mathrm{C}\right.$ e $\left.1994=22,8^{\circ} \mathrm{C}\right)$.

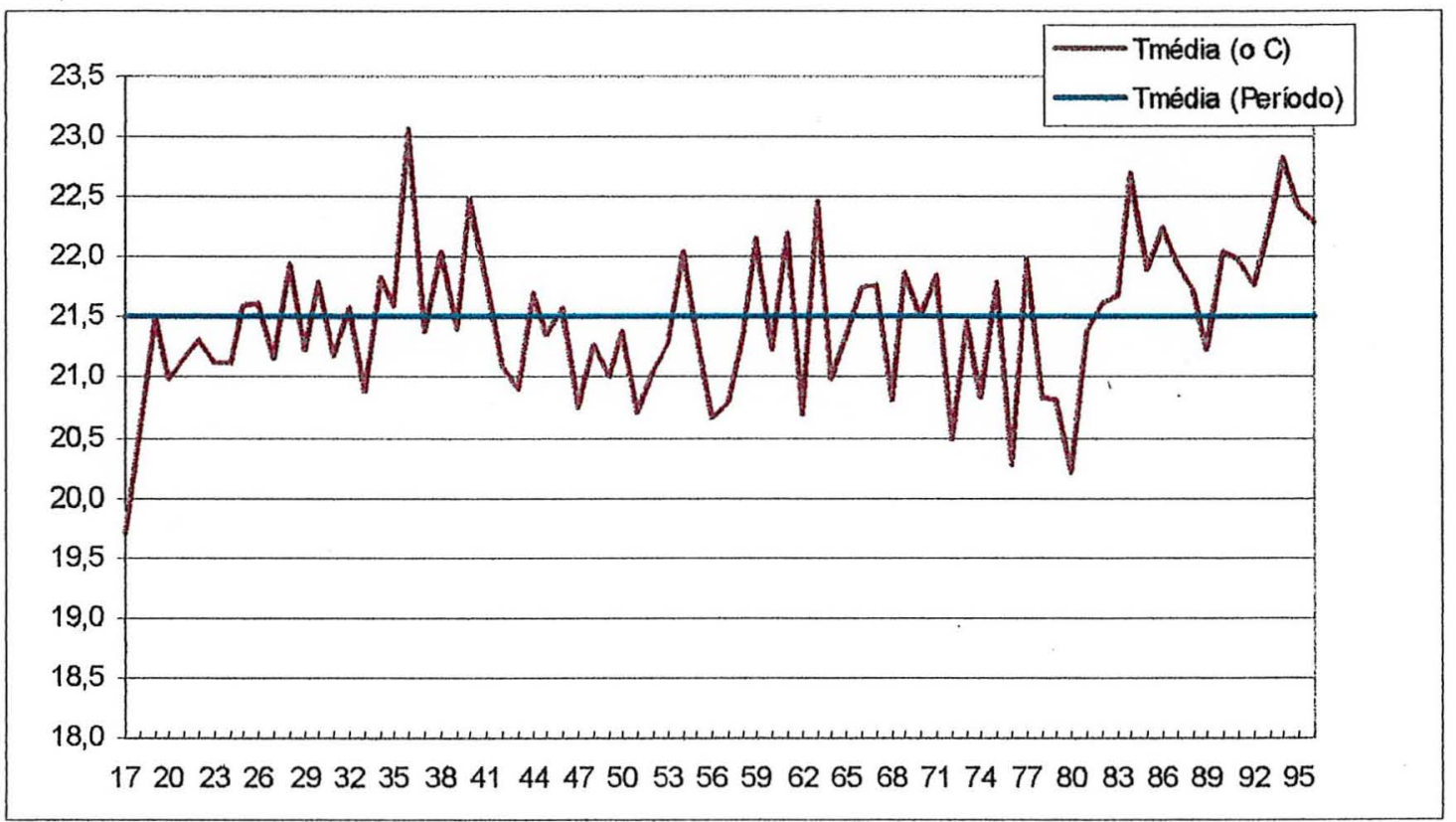

Figura 5- Valores da temperatura média anual da área de estudo. Período: 1917-1996

\subsection{Distribuição mensal e decendial das probabilidades da temperatura média e da precipitação pluvial da área de estudo (Período: 1917-1996)}

Na Tabela 9, verificou-se que, para a probabilidade de $20 \%$ de ocorrência, quanto à temperatura média mensal na estação chuvosa (outubro a março), os valores foram menores ou iguais a $23,8^{\circ} \mathrm{C}$ e menores ou iguais a $23,5^{\circ} \mathrm{C}, 23,7^{\circ} \mathrm{C}$ e $23,3^{\circ} \mathrm{C}$ respectivamente, no $1^{\circ}, 2^{\circ}$ e $3^{\circ}$ decêndios (Tabela 10). Para a probabilidade de $80 \%$, ocorreram valores menores ou iguais $25,3^{\circ} \mathrm{C}$ e, no $1^{\circ}, 2^{\circ}$ e $3^{\circ}$ decêndios, valores menores ou iguais a $25,7^{\circ} \mathrm{C}, 25,9^{\circ} \mathrm{C}$ e $25,7^{\circ} \mathrm{C}$, respectivamente. $\mathrm{Na}$ estação seca (abril a setembro), para $20 \%$ de ocorrência, os valores foram menores ou iguais a $22,4^{\circ} \mathrm{C}$ e menores ou iguais a $21,5^{\circ} \mathrm{C}, 22,3^{\circ} \mathrm{C}$ e $22,1^{\circ} \mathrm{C}$ nos três decêndios e para $80 \%$, valores menores ou iguais a $23,8^{\circ} \mathrm{C}$ e $24,2^{\circ} \mathrm{C}$ para os três decêndios, respectivamente. A média e mediana das temperaturas médias mensais e decendiais apresentaram-se muito 
próximas, diferindo aproximadamente em $0,3^{\circ} \mathrm{C}$.

Tabela 9- Probabilidades (\%) da temperatura média mensal, com 10, 20, 30, 40, 50, 60, 70, 80, 90 e 99\% de ocorrências na área de estudo entre 1917-1996, com valores menores ou iguais, referentes aos dados abaixo.

\begin{tabular}{|c|c|c|c|c|c|c|c|c|c|c|c|c|}
\hline \multirow{3}{*}{ PROB (\%) } & \multicolumn{12}{|c|}{ Temperaturas médias mensais $\left({ }^{\circ} \mathrm{C}\right)$} \\
\hline & \multicolumn{11}{|c|}{ Meses } & \\
\hline & JAN & FEV & MAR & ABR & MAI & JUN & JUL & AGO & SET & OUT & NOV & DEZ \\
\hline 10 & 23,2 & 23,3 & 23,0 & 20,3 & 17,6 & 16,2 & 15,5 & 17,6 & 19,3 & 20,8 & 21,7 & 22,6 \\
\hline 20 & 23,7 & 23,8 & 23,4 & 20,7 & 18,3 & 16,5 & 16,1 & 18,1 & 19,6 & 21,4 & 22,4 & 23,1 \\
\hline 30 & 23,9 & 24,0 & 23,5 & 21,1 & 18,4 & 17,1 & 16,6 & 18,4 & 19,8 & 21,7 & 22,6 & 23,3 \\
\hline 40 & 24,2 & 24,2 & 23,8 & 21,5 & 18,7 & 17,3 & 17,1 & 18,8 & 20,4 & 21,9 & 22,8 & 23,6 \\
\hline 50 & 24,4 & 24,6 & 24,0 & 21,9 & 19,1 & 17,6 & 17,3 & 19,1 & 20,7 & 22,1 & 23,0 & 23,8 \\
\hline 60 & 24,5 & 24,7 & 24,3 & 22,0 & 19,2 & 17,8 & 17,5 & 19,3 & 20,9 & 22,3 & 23,3 & 24,0 \\
\hline 70 & 24,7 & 25,0 & 24,5 & 22,4 & 19,7 & 18,1 & 18,0 & 19,7 & 21,1 & 22,8 & 23,5 & 24,2 \\
\hline 80 & 25,2 & 25,3 & 24,7 & 23,1 & 20,1 & 18,4 & 18,4 & 20,0 & 21,5 & 23,1 & 23,8 & 24,5 \\
\hline 90 & 25,5 & 25,8 & 24,9 & 23,3 & 20,6 & 18,9 & 19,1 & 20,4 & 22,2 & 23,7 & 24,6 & 24,9 \\
\hline 99,9 & 26,4 & 27,5 & 25,9 & 24,1 & 22,5 & 20,3 & 19,8 & 23,9 & 24,0 & 25,3 & 25,7 & 26,5 \\
\hline MEDIA & 24,6 & 24,8 & 24,2 & 22,0 & 19,4 & 17,8 & 17,5 & 19,5 & 21,0 & 22,5 & 23,3 & 24,1 \\
\hline MEDIANA & 24,5 & 24,7 & 24,2 & 22,0 & 19,2 & 17,7 & 17,4 & 19,2 & 20,8 & 22,2 & 23,2 & 23,9 \\
\hline
\end{tabular}

Tabela 10- Probabilidades (\%) da temperatura média decendial, com 10, 20, 30, 40, 50, 60, 70, 80, 90 e 99\% de ocorrências, na área de estudo entre 1917-1996, com valores menores ou iguais, referentes aos dados abaixo.

\begin{tabular}{|c|c|c|c|c|c|c|c|c|c|c|c|c|}
\hline \multirow{4}{*}{ PROB (\%) } & \multicolumn{12}{|c|}{ Temperaturas médias ( $\left.{ }^{\circ} \mathrm{C}\right)$} \\
\hline & \multicolumn{11}{|c|}{ Meses } & \multirow[b]{2}{*}{ DEZ } \\
\hline & JAN & FEV & MAR & ABR & MAI & JUN & JUL & AGO & SET & OUT & NOV & \\
\hline & \multicolumn{11}{|c|}{$1^{\circ}$ Decêndio } & \\
\hline 10 & 22,4 & 23,0 . & 23,3 & 20,7 & 17,3 & 15,2 & 15,0 & 16,3 & 18,1 & 19,8 & 21,0 & 22,4 \\
\hline 20 & 23,2 & 23,4 & 23,5 & 21,2 & 18,3 & 16,0 & 15,9 & 17,0 & 18,8 & 20,5 & 21,5 & 23,0 \\
\hline 30 & 23,6 & 23,7 & 23,7 & 21,9 & 19,1 & 16,5 & 16,6 & 17,8 & 19,1 & 21,0 & 22,1 & 23,4 \\
\hline 40 & 23,9 & 23,9 & 24,1 & 22,1 & 19,4 & 16,9 & 17,0 & 18,2 & 19,6 & 21,4 & 22,3 & 23,6 \\
\hline 50 & 24,4 & 24,2 & 24,5 & 22,5 & 19,8 & 17,3 & 17,3 & 18,6 & 19,9 & 21,8 & 22,8 & 23,8 \\
\hline 60 & 24,5 & 24,4 & 24,7 & 23,0 & 20,1 & 18,0 & 17,7 & 19,0 & 20,3 & 22,6 & 23,3 & 24,2 \\
\hline 70 & 24,9 & 24,9 & 24,9 & 23,3 & 20,6 & 18,6 & 18,0 & 19,2 & 20,8 & 22,9 & 23,8 & 24,3 \\
\hline 80 & 25,7 & 25,6 & 25,3 & 24,0 & 21,0 & 19,2 & 18,5 & 19,9 & 21,6 & 23,8 & 24,2 & 25,1 \\
\hline 90 & 26,1 & 26,3 & 25,6 & 24,6 & 22,0 & 19,7 & 19,3 & 20,5 & 22,4 & 24,4 & 25,1 & 25,4 \\
\hline 99,9 & 26,8 & 27,4 & 26,5 & 26,0 & 23,7 & 22,2 & 20,5 & 21,9 & 24,5 & 27,1 & 27,2 & 26,5 \\
\hline MEDIA & 24,6 & 24,7 & 24,6 & 22,9 & 20,1 & 18,0 & 17,6 & 18,8 & 20,5 & 22,5 & 23,3 & 24,2 \\
\hline MEDIANA & 24,5 & 24,3 & 24,6 & 22,8 & 20,0 & 17,7 & 17,5 & 18,8 & 20,1 & 22,2 & 23,1 & 24,0 \\
\hline
\end{tabular}


Continuação:

Tabela 10- Probabilidades (\%) da temperatura média decendial, com 10, 20, 30, 40, 50, $60,70,80,90$ e 99\% de ocorrências, na área de estudo entre 1917-1996, com valores menores ou iguais, referentes aos dados abaixo.

\begin{tabular}{|c|c|c|c|c|c|c|c|c|c|c|c|c|}
\hline \multirow{4}{*}{ PROB (\%) } & \multicolumn{11}{|c|}{ Temperaturas médias $\left({ }^{\circ} \mathrm{C}\right)$} & \multirow[b]{3}{*}{ DEZ } \\
\hline & \multicolumn{10}{|c|}{ Meses } & \multirow{3}{*}{ NOV } & \\
\hline & JAN & FEV & MAR & ABR & MAI & JUN & JUL & AGO & SET & OUT & & \\
\hline & \multicolumn{11}{|c|}{$2^{\circ}$ decêndio } & \\
\hline 10 & 22,6 & 23,2 & 22,6 & 20,1 & 16,9 & 15,5 & 13,9 & 16,5 & 18,1 & 19,7 & 21,5 & 22,4 \\
\hline 20 & 23,2 & 23,7 & 23,0 & 20,6 & 17,9 & 16,2 & 15,5 & 17,7 & 19,2 & 20,4 & 22,3 & 23,0 \\
\hline 30 & 23,5 & 23,9 & 23,3 & 21,1 & 18,7 & 16,8 & 16,0 & 18,5 & 20,0 & 21,0 & 22,7 & 23,4 \\
\hline 40 & 24,0 & 24,2 & 23,9 & 21,3 & 19,1 & 17,1 & 16,4 & 18,8 & 20,2 & 21,4 & 22,8 & 23,6 \\
\hline 50 & 24,3 & 24,5 & 24,1 & 21,7 & 19,5 & 17,7 & 17,0 & 19,0 & 20,6 & 21,9 & 23,2 & 23,8 \\
\hline 60 & 24,5 & 25,0 & 24,4 & 22,1 & 19,9 & 18,0 & 17,6 & 19,5 & 21,3 & 22,3 & 23,4 & 24,2 \\
\hline 70 & 24,7 & 25,3 & 24,7 & 22,6 & 20,2 & 18,6 & 18,2 & 20,0 & 21,6 & 23,0 & 23,8 & 24,3 \\
\hline 80 & 25,3 & 25,9 & 25,0 & 23,0 & 20,8 & 19,1 & 18,8 & 20,5 & 22,4 & 23,5 & 24,2 & 25,1 \\
\hline 90 & 26,3 & 26,5 & 25,5 & 23,4 & 21,5 & 19,5 & 19,6 & 21,1 & 23,0 & 24,0 & 24,6 & 25,4 \\
\hline 99,9 & 27,7 & 27,3 & 26,3 & 25,2 & 22,4 & 20,5 & 20,9 & 22,1 & 24,9 & 26,5 & 27,5 & 26,5 \\
\hline MEDIA & 24,6 & 25,0 & 24,3 & 22,1 & 19,7 & 17,9 & 17,4 & 19,4 & 21,1 & 22,4 & 23,6 & 24,2 \\
\hline MEDIANA & 24,4 & 24,8 & 24,3 & 21,9 & 19,7 & 17,9 & 17,3 & 19,3 & 21,0 & 22,1 & 23,3 & 24,0 \\
\hline PROB (\%) & \multicolumn{12}{|c|}{$3^{\circ}$ Decêndio } \\
\hline 10 & 22,8 & 22,5 & 21,9 & 18,7 & 16,1 & 15,3 & 15,0 & 17,5 & 19,1 & 20,7 & 21,6 & 22,3 \\
\hline 20 & 23,2 & 23,3 & 22,6 & 19,6 & 16,9 & 16,4 & 16,2 & 18,2 & 19,9 & 21,3 & 22,1 & 22,9 \\
\hline 30 & 23,8 & 23,9 & 23,0 & 20,2 & 17,3 & 16,9 & 16,9 & 18,7 & 20,4 & 21,6 & 22,4 & 23,2 \\
\hline 40 & 24,2 & 24,1 & 23,2 & 20,7 & 17.7 & 17,5 & 17,2 & 19,0 & 20,7 & 21,9 & 22,8 & 23,5 \\
\hline 50 & 24,4 & 24,5 & 23,5 & 20,9 & 17,9 & 17,9 & 17,7 & 19,4 & 21,1 & 22,3 & 23,2 & 23,8 \\
\hline 60 & 24,6 & 25,0 & 23,9 & 21,3 & 18,6 & 18,1 & 18,2 & 19,9 & 21,6 & 22,6 & 23,5 & 24,1 \\
\hline 70 & 24,8 & 25,3 & 24,1 & 21,9 & 19,2 & 18,4 & 18,5 & 20,6 & 22,0 & 23,1 & 23,9 & 24,4 \\
\hline 80 & 25,5 & 25,7 & 24,5 & 22,6 & 20,1 & 18,7 & 18,9 & 21,4 & 22,5 & 24,0 & 24,3 & 24,6 \\
\hline 90 & 26,2 & 26,1 & 24,8 & 23,1 & 20,4 & 19,3 & 19,3 & 21,9 & 23,0 & 24,5 & 24,9 & 25,3 \\
\hline 99,9 & 27,5 & 27,6 & 25,9 & 26,8 & 21,8 & 20,3 & 20,9 & 23,9 & 25,4 & 25,4 & 25,9 & 26,0 \\
\hline MEDIA & 24,7 & 24,8 & 23,7 & 21,6 & 18,6 & 17,9 & 17,9 & 20,1 & 21,6 & 22,7 & 23,5 & 24,0 \\
\hline MEDIANA & 24,5 & 24,8 & 23,7 & 21,1 & 18,3 & 18,0 & 18,0 & 19,7 & 21,4 & 22,5 & 23,4 & 24,0 \\
\hline
\end{tabular}

$\mathrm{Na}$ distribuição das probabilidades em $20 \%$, das precipitações pluviais mensais, representada pela (Tabela 11), verificou-se que, na estação chuvosa, os valores foram menores ou iguais a $139 \mathrm{~mm}$ e 27,29 e $34 \mathrm{~mm}$ nos $1^{\circ}, 2^{\circ}$ e $3^{\circ}$ decêndios, respectivamente (Tabela 12), enquanto que, para a probabilidade de $80 \%$, esses valores foram abaixo ou iguais a $296 \mathrm{~mm}$ e $129,137,119 \mathrm{~mm}$ nos $1^{\circ}, 2^{\circ}$ e $3^{\circ}$ decêndios. $\mathrm{Na}$ estação seca ficou abaixo de $16 \mathrm{~mm}$ para probabilidade de $20 \%$ e 2,0 e $2 \mathrm{~mm}$ nos $1^{\circ}, 2^{\circ}$ e $3^{\circ}$ decêndios, respectivamente e $103 \mathrm{~mm}$ para $80 \%$ e 44,4 e $42 \mathrm{~mm}$ nos $1^{\circ}, 2^{\circ}$ e $3^{\circ}$ decêndios. A média apresentou valores $13 \mathrm{~mm}$ maiores na estação chuvosa e $17 \mathrm{~mm}$ na 
estação seca em relação à mediana.

Tabela 11- Probabilidades (\%) da precipitação média mensal, com 10, 20, 30, 40, 50, 60, $70,80,90$ e $99 \%$ de ocorrências, com valores menores ou iguais, referentes aos dados abaixo.

\begin{tabular}{|c|c|c|c|c|c|c|c|c|c|c|c|c|}
\hline \multirow{3}{*}{ PROB (\%) } & \multicolumn{12}{|c|}{ Precipitacōes pluviais mensais (mm) } \\
\hline & \multicolumn{11}{|c|}{ Meses } & \multirow[b]{2}{*}{ DEZ } \\
\hline & JAN & FEV & MAR & ABR & MA & JUN & JUL & AGO & SET & OUT & NOV & \\
\hline 10 & 109 & 64 & 42 & 16 & 4 & 1 & 0 & 0 & 6 & 43 & 49 & 106 \\
\hline 20 & 132 & 96 & 66 & 22 & 13 & 13 & 2 & 4 & 16 & 60 & 82 & 139 \\
\hline 30 & 158 & 119 & 92 & 32 & 22 & 19 & 7 & 6 & 24 & 70 & 92 & 154 \\
\hline 40 & 201 & 144 & 113 & 39 & 34 & 24 & 11 & 14 & 38 & 87 & 110 & 181 \\
\hline 50 & 220 & 177 & 140 & 53 & 42 & 32 & 15 & 20 & 53 & 101 & 124 & 193 \\
\hline 60 & 257 & 203 & 160 & 67 & 49 & 41 & 21 & 28 & 62 & 116 & 133 & 199 \\
\hline 70 & 272 & 221 & 183 & 83 & 63 & 48 & 29 & 37 & 73 & 127 & 156 & 220 \\
\hline 80 & 296 & 259 & 196 & 103 & 78 & 66 & 48 & 52 & 91 & 162 & 196 & 261 \\
\hline 90 & 324 & 328 & 226 & 126 & 99 & 97 & $\pi$ & 72 & 129 & 197 & 218 & 314 \\
\hline 99,9 & 491 & 462 & 432 & 239 & 250 & 187 & 145 & 133 & 257 & 255 & 260 & 421 \\
\hline MEDIA & 246 & 207 & 165 & 78 & 65 & 53 & 35 & 36 & 75 & 122 & 142 & 219 \\
\hline MEDIANA & 238 & 190 & 150 & 60 & 46 & 36 & 18 & 24 & 57 & 109 & 129 & 196 \\
\hline
\end{tabular}

Tabela 12- Probabilidades (\%) da precipitação média mensal decendial, para o $1^{\circ}, 2^{\circ}$ e $3^{\circ}$, com $10,20,30,40,50,60,70,80,90$ e $99 \%$ de ocorrências na área de estudo entre 1917-1996, com valores menores ou iguais, referentes aos dados abaixo.

\begin{tabular}{|c|c|c|c|c|c|c|c|c|c|c|c|c|}
\hline \multirow{4}{*}{ PROB (\%) } & \multicolumn{12}{|c|}{ Precipitaçöes pluviais decendiais mensais (mm) } \\
\hline & \multicolumn{10}{|c|}{ Meses } & \multirow{3}{*}{ Nov } & \multirow{3}{*}{ DEZ } \\
\hline & JAN & FEV & MAR & ABR & MAI & JUN & JUL & AGO & SET & OUT & & \\
\hline & \multicolumn{10}{|c|}{10 Decêndio } & & \\
\hline 10 & 16 & 7 & 1 & 0 & 0 & 0 & 0 & 0 & 0 & 0 & 3 & 9 \\
\hline 20 & 25 & 27 & 10 & 2 & 0 & 0 & 0 & 0 & 0 & 2 & 11 & 16 \\
\hline 30 & 39 & 41 & 18 & 5 & 1 & 0 & 0 & 0 & 0 & 7 & 17 & 28 \\
\hline 40 & 50 & 52 & 28 & 12 & 4 & 1 & 0 & 0 & 1 & 14 & 27 & 35 \\
\hline 50 & 56 & 64 & 39 & 19 & 10 & 4 & 2 & 1 & 7 & 24 & 34 & 51 \\
\hline 60 & 66 & 72 & 51 & 30 & 17 & 13 & 4 & 2 & 10 & 35 & 41 & 56 \\
\hline 70 & 83 & 89 & 73 & 35 & 24 & 19 & 8 & 7 & 15 & 41 & 51 & 72 \\
\hline 80 & 123 & 117 & 85 & 44 & 35 & 30 & 13 & 15 & 34 & 57 & 57 & 90 \\
\hline 90 & 145 & 171 & 109 & 57 & 45 & 38 & 30 & 24 & 49 & 75 & 63 & 122 \\
\hline 99,9 & 227 & 278 & 220 & 125 & 75 & 161 & 78 & 71 & 119 & 155 & 107 & 191 \\
\hline MEDIA & 83 & 92 & $\tilde{3}$ & 33 & 21 & 27 & 13 & 12 & 23 & 41 & 41 & 67 \\
\hline MEDIANA & 61 & 68 & 45 & 24 & 14 & 9 & 3 & 1 & 8 & 29 & 37 & 54 \\
\hline
\end{tabular}


Continuação:

Tabela 12- Probabilidades (\%) da precipitação média mensal decendial, para $01^{\circ}, 2^{\circ}$ e $3^{\circ}$, com $10,20,30,40,50,60,70,80,90$ e 99\% de ocorrências na área de estudo entre 1917-1996, com valores menores ou iguais, referentes aos dados abaixo.

\begin{tabular}{|c|c|c|c|c|c|c|c|c|c|c|c|c|}
\hline \multirow{3}{*}{ PROB (\%) } & \multicolumn{12}{|c|}{ Precipitações pluviais decendiais mensais (mm) } \\
\hline & JAN & FEV & MAR & ABR & MAI & JUN & JUL & AGO & SET & OUT & NOV & DEZ \\
\hline & \multicolumn{11}{|c|}{20 Decêndio } & \\
\hline 10 & 14 & 13 & 4 & 0 & 0 & 0 & 0 & 0 & 0 & 0 & 6 & 13 \\
\hline 20 & 25 & 20 & 20 & 0 & 0 & 0 & 0 & 0 & 0 & 10 & 11 & 29 \\
\hline 30 & 45 & 34 & 26 & 2 & 0 & 0 & 0 & 0 & 1 & 17 & 20 & 44 \\
\hline 40 & 55 & 42 & 33 & 5 & 3 & 0 & 0 & 0 & 4 & 25 & 27 & 54 \\
\hline 50 & 69 & 52 & 39 & 9 & 9 & 2 & 0 & 0 & 9 & 31 & 35 & 60 \\
\hline 60 & 86 & 62 & 46 & 17 & 16 & 10 & 1 & 3 & 21 & 40 & 41 & 67 \\
\hline 70 & 109 & 74 & 58 & 23 & 21 & 15 & 4 & 7 & 31 & 50 & 47 & 74 \\
\hline 80 & 137 & 96 & 71 & 35 & 29 & 32 & 14 & 11 & 43 & 66 & 75 & 97 \\
\hline 90 & 154 & 117 & 86 & 62 & 40 & 43 & 24 & 22 & 64 & 87 & 95 & 126 \\
\hline 99,9 & 331 & 242 & 269 & 126 & 154 & 155 & 96 & 73 & 151 & 134 & 176 & 322 \\
\hline MEDIA & 102 & 75 & 65 & 28 & 27 & 26 & 14 & 12 & 32 & 46 & 53 & 89 \\
\hline MEDIANA & 77 & 57 & 42 & 13 & 12 & 6 & 0 & 2 & 15 & 36 & 38 & 63 \\
\hline PROB (\%) & \multicolumn{11}{|c|}{30 Decêndio } & \\
\hline 10 & 18 & 3 & 0 & 0 & 0 & 0 & 0 & 0 & 0 & 1 & 5 & 15 \\
\hline 20 & 34 & 12 & 2 & 0 & 0 & 0 & 0 & 0 & 2 & 10 & 14 & 28 \\
\hline 30 & 46 & 23 & 9 & 0 & 0 & 0 & 0 & 0 & 8 & 16 & 23 & 39 \\
\hline 40 & 53 & 33 & 18 & 2 & 1 & 0 & 0 & 1 & 11 & 23 & 28 & 47 \\
\hline 50 & 65 & 43 & 27 & 10 & 4 & 0 & 0 & 3 & 14 & 29 & 43 & 62 \\
\hline 60 & 78 & 50 & 44 & 12 & 7 & 3 & 1 & 8 & 19 & 41 & 57 & 74 \\
\hline 70 & 83 & 58 & 56 & 16 & 13 & 10 & 5 & 13 & 24 & 46 & 65 & 87 \\
\hline 80 & 105 & 82 & 73 & 23 & 21 & 18 & 8 & 25 & 42 & 61 & 82 & 119 \\
\hline 90 & 126 & 111 & 107 & 36 & 39 & 27 & 23 & 49 & 55 & 90 & 103 & 155 \\
\hline 99,9 & 261 & 190 & 182 & 110 & 176 & 156 & 85 & 99 & 132 & 134 & 162 & 183 \\
\hline MEDIA & 87 & 60 & 52 & 21 & 26 & 21 & 12 & 20 & 31 & 45 & 58 & 81 \\
\hline MEDIANA & 72 & 47 & 36 & 11 & 5 & 2 & 1 & 6 & 16 & 35 & 50 & 68 \\
\hline
\end{tabular}


4.6 Distribuição mensal e decendial das probabilidades da evapotranspiração potencial, armazenamento hídrico, evapotranspiração real, deficiência hídrica e excedente hídrico para CAD: 50,100 e $150 \mathrm{~mm}$ da área de estudo, entre 19171996.

Os dados apresentados na Tabela 13, referem-se aos valores mensais da evapotranspiração potencial mensal, calculada através do balanço hídrico seqüencial mensal para as CAD: 50,100 e $150 \mathrm{~mm}$.

Tabela 13- Probabilidades (\%) da evapotranspiração potencial mensal, com $10,20,30$, $40,50,60,70,80,90$ e $99 \%$ de ocorrências na área de estudo entre 19171996, com CAD: 50,100 e $150 \mathrm{~mm}$, com valores menores ou iguais, referentes aos dados abaixo.

\begin{tabular}{|c|c|c|c|c|c|c|c|c|c|c|c|c|}
\hline \multirow[b]{2}{*}{$\operatorname{PROB}(\%)$} & \multicolumn{12}{|c|}{ Evapotranspiraçậo potencial mensal (mm) - CAD: 50 mm } \\
\hline & JAN & FEV & MAR & ABR & MAI & JUN & JUL & AGO & SET & OUT & NOV & DEZ \\
\hline 10 & 113 & 99 & 101 & 69 & 49 & 38 & 36 & 50 & 63 & 82 & 92 & 107 \\
\hline 20 & 119 & 104 & 105 & 72 & 53 & 40 & 39 & 53 & 66 & 88 & 99 & 113 \\
\hline 30 & 121 & 106 & 106 & 76 & 54 & 43 & 42 & 56 & 67 & 91 & 101 & 115 \\
\hline 40 & 125 & 108 & 110 & 79 & 56 & 44 & 45 & 58 & 72 & 93 & 103 & 119 \\
\hline 50 & 127 & 112 & 112 & 83 & 59 & 46 & 46 & 61 & 75 & 95 & 106 & 121 \\
\hline 60 & 128 & 113 & 115 & 83 & 59 & 47 & 47 & 62 & 76 & 97 & 109 & 123 \\
\hline 70 & 131 & 117 & 117 & 87 & 63 & 49 & 51 & 65 & 78 & 102 & 111 & 126 \\
\hline 80 & 137 & 120 & 119 & 93 & 66 & 51 & 53 & 67 & 82 & 105 & 114 & 129 \\
\hline 90 & 141 & 126 & 122 & 95 & 70 & 54 & 58 & 71 & 88 & 112 & 123 & 134 \\
\hline 99,9 & 153 & 142 & 133 & 103 & 99 & 64 & 63 & 102 & 105 & 130 & 137 & 156 \\
\hline MEDIA & 129 & 115 & 114 & 84 & 63 & 48 & 48 & 65 & 77 & 99 & 110 & 124 \\
\hline MEDIANA & 128 & 113 & 113 & 83 & 59 & 47 & 47 & 61 & 76 & 96 & 107 & 122 \\
\hline \multirow[t]{2}{*}{ PROB $(\%)$} & \multicolumn{12}{|c|}{ CAD: $100 \mathrm{~mm}$} \\
\hline & JAN & FEV & MAR & ABR & MA & JUN & JUL & $A G O$ & SET & OUT & NOV & DEZ \\
\hline 10 & 113 & 99 & 101 & 69 & 49 & 38 & 36 & so & 63 & 82 & 92 & 107 \\
\hline 20 & 119 & 104 & 105 & 72 & 53 & 40 & 39 & 53 & 66 & 88 & 99 & 113 \\
\hline 30 & 121 & 106 & 106 & 76 & 54 & 43 & 42 & 56 & 67 & 91 & 101 & 115 \\
\hline 40 & 125 & 108 & 110 & 79 & 56 & 44 & 45 & 58 & 72 & 93 & 103 & 119 \\
\hline 50 & 127 & 112 & 112 & 83 & 59 & 46 & 46 & 61 & 75 & 95 & 106 & 121 \\
\hline 60 & 128 & 113 & 115 & 83 & 59 & 47 & 47 & 62 & 76 & 97 & 109 & 123 \\
\hline 70 & 131 & 117 & 117 & 87 & 63 & 49 & 51 & 65 & 78 & 102 & 111 & 126 \\
\hline 80 & 137 & 120 & 119 & 93 & 66 & 51 & 53 & 67 & 82 & 105 & 114 & 129 \\
\hline 90 & 141 & 126 & 122 & 95 & 70 & 54 & 58 & 71 & 88 & 112 & 123 & 134 \\
\hline 99,9 & 153 & 142 & 133 & 103 & 127 & 64 & 63 & 102 & 105 & 130 & 137 & 156 \\
\hline MEDIA & 129 & 115 & 114 & 84 & 66 & 48 & 48 & 65 & 77 & 99 & 110 & 124 \\
\hline MEDIANA & 128 & 113 & 113 & 83 & 59 & 47 & 47 & 61 & 76 & 96 & 107 & 122 \\
\hline
\end{tabular}


Continuação:

Tabela 13- Probabilidades (\%) da evapotranspiração potencial mensal, com 10, 20, 30, $40,50,60,70,80,90$ e $99 \%$ de ocorrências na área de estudo entre 1917 1996, com CAD: 50, 100 e $150 \mathrm{~mm}$, com valores menores ou iguais, referentes aos dados abaixo.

\begin{tabular}{|c|c|c|c|c|c|c|c|c|c|c|c|c|}
\hline \multirow[b]{2}{*}{ PROB (\%) } & \multicolumn{12}{|c|}{ CAD: $150 \mathrm{~mm}$} \\
\hline & JAN & FEV & MAR & ABR & MA! & JUN & JUL & ACO & SET & OUT & Nov & $D E Z$ \\
\hline 10 & 113 & 99 & 101 & 69 & 49 & 38 & 36 & 50 & 63 & 82 & 92 & 107 \\
\hline 20 & 119 & 104 & 105 & 72 & 53 & 40 & 39 & 53 & 66 & 88 & 99 & 113 \\
\hline 30 & 121 & 106 & 106 & 76 & 54 & 43 & 42 & 56 & 67 & 91 & 101 & 115 \\
\hline 40 & 125 & 108 & 110 & 79 & 56 & 44 & 45 & 58 & 72 & 93 & 103 & 119 \\
\hline 50 & 127 & 112 & 112 & 83 & 59 & 46 & 46 & 61 & 75 & 95 & 106 & 121 \\
\hline 60 & 128 & 113 & 115 & 83 & 59 & 47 & 47 & 62 & 76 & 97 & 109 & 123 \\
\hline 70 & 131 & 117 & 117 & 87 & 63 & 49 & 51 & 65 & 78 & 102 & 111 & 126 \\
\hline 80 & 137 & 120 & 119 & 93 & 66 & 51 & 53 & 67 & 82 & 105 & 114 & 129 \\
\hline 90 & 141 & 126 & 122 & 95 & 70 & 54 & 58 & 71 & 88 & 112 & 123 & 134 \\
\hline 99,9 & 153 & 142 & 133 & 103 & 127 & 64 & 63 & 102 & 105 & 130 & 137 & 156 \\
\hline MEDHA & 129 & 115 & 114 & 84 & 66 & 48 & 48 & 65 & 77 & 99 & 110 & 124 \\
\hline MEDAANA & 128 & 113 & 113 & 83 & 59 & 47 & 47 & 61 & 76 & 96 & 107 & 122 \\
\hline
\end{tabular}

A evapotranspiração potencial para os três níveis de CAD, com $20 \%$ de probabilidades na estação chuvosa, apresentou valores iguais ou menores a $119 \mathrm{~mm}$ e, com $80 \%$ de probabilidades, valores menores de $137 \mathrm{~mm}$. Para a estação seca, os valores abaixo de $72 \mathrm{~mm}$ ocorreram com $20 \%$ de probabilidades e abaixo de $93 \mathrm{~mm}$ para $80 \%$. A média e mediana tiveram os valores muitos próximos diferindo, aproximadamente, em 4 mm.

Nos três decêndios das CAD: 50,100 e 150 mm (Tabela 14, 15 e 16), constatouse que, na estação chuvosa, os valores abaixo de $39 \mathrm{~mm}$ apresentaram $20 \%$ de probabilidades e $49 \mathrm{~mm}$ para $80 \%$ de probabilidades e na estação seca com $20 \%$ de probabilidades os valores foram abaixo de $26 \mathrm{~mm}$ e $34 \mathrm{~mm}$ para $80 \%$ de probabilidades. 
Tabela 14- Probabilidades (\%) da evapotranspiração potencial decendial, com 10, 20, 30, $40,50,60,70,80,90$ e $99 \%$ de ocorrências na área de estudo entre 19171996, com CAD: 50mm, com valores menores ou iguais, referentes aos dados abaixo.

\begin{tabular}{|c|c|c|c|c|c|c|c|c|c|c|c|c|}
\hline \multirow{4}{*}{ PROB (\%) } & \multicolumn{12}{|c|}{ Evapotranspiração potencial (mm) - CAD: $50 \mathrm{~mm}$} \\
\hline & \multicolumn{12}{|c|}{ Meses } \\
\hline & JAN & FEV & MAR & ABR & MAI & JUN & JUL & AGO & SET & OUT & NOV & DEZ \\
\hline & \multicolumn{12}{|c|}{$1^{0}$ Decêndio } \\
\hline 10 & 33 & 34 & 33 & 24 & 15 & 10 & 10 & 13 & 17 & 22 & 27 & 32 \\
\hline 20 & 36 & 35 & 35 & 26 & 17 & 12 & 12 & 14 & 19 & 24 & 29 & 34 \\
\hline 30 & 37 & 37 & 35 & 28 & 19 & 13 & 13 & 16 & 20 & 26 & 31 & 35 \\
\hline 40 & 38 & 38 & 37 & 28 & 19 & 14 & 14 & 17 & 21 & 27 & 32 & 36 \\
\hline 50 & 40 & 38 & 38 & 30 & 20 & 14 & 14 & 18 & 22 & 29 & 33 & 38 \\
\hline 60 & 41 & 39 & 39 & 31 & 21 & 16 & 15 & 18 & 23 & 31 & 35 & 39 \\
\hline 70 & 42 & 41 & 40 & 32 & 22 & 17 & 16 & 19 & 24 & 32 & 37 & 41 \\
\hline 80 & 47 & 45 & 41 & 34 & 24 & 18 & 17 & 21 & 26 & 35 & 39 & 44 \\
\hline 90 & 48 & 47 & 42 & 37 & 26 & 20 & 18 & 22 & 29 & 38 & 42 & 46 \\
\hline 99,9 & 51 & 51 & 47 & 42 & 31 & 26 & 21 & 48 & 36 & 48 & 51 & 55 \\
\hline MEDIA & 41 & 41 & 39 & 31 & 21 & 16 & 15 & 21 & 24 & 31 & 36 & 40 \\
\hline \multirow[t]{2}{*}{ MEDIANA } & 41 & 39 & 38 & 30 & 21 & 15 & 15 & 18 & 22 & 30 & 34 & 38 \\
\hline & \multicolumn{12}{|c|}{$2^{\circ}$ Decêndio } \\
\hline PROB (\%) & JAN & FEV & MAR & ABR & MAI & JUN & JUL & AGO & SET & OUT & NOV & DEZ \\
\hline 10 & 33 & 34 & 31 & 22 & 14 & 11 & 8 & 13 & 18 & 23 & 30 & 33 \\
\hline 20 & 36 & 36 & 32 & 23 & 16 & 12 & 11 & 16 & 20 & 25 & 32 & 35 \\
\hline 30 & 37 & 37 & 33 & 25 & 17 & 13 & 12 & 18 & 22 & 27 & 33 & 37 \\
\hline 40 & 39 & 38 & 35 & 25 & 18 & 14 & 13 & 18 & 23 & 28 & 34 & 38 \\
\hline 50 & 40 & 39 & 36 & 27 & 19 & 15 & 14 & 19 & 24 & 29 & 35 & 38 \\
\hline 60 & 41 & 41 & 37 & 28 & 20 & 16 & 15 & 20 & 26 & 31 & 36 & 40 \\
\hline 70 & 42 & 42 & 38 & 29 & 21 & 17 & 16 & 21 & 27 & 33 & 38 & 41 \\
\hline 80 & 44 & 45 & 39 & 30 & 23 & 18 & 18 & 22 & 29 & 35 & 39 & 43 \\
\hline 90 & 48 & 48 & 41 & 31 & 24 & 19 & 19 & 24 & 31 & 37 & 41 & 45 \\
\hline 99,9 & 54 & 50 & 45 & 38 & 27 & 21 & 23 & 27 & 38 & 47 & 52 & 50 \\
\hline MEDIA & 41 & 41 & 37 & 28 & 20 & 15 & 15 & 20 & 26 & 31 & 37 & 40 \\
\hline MEDLANA & 41 & 40 & 36 & 27 & 20 & 15 & 14 & 19 & 25 & 30 & 35 & 39 \\
\hline
\end{tabular}


Continuação:

Tabela 14- Probabilidades (\%) da evapotranspiração potencial decendial, com 10, 20,30, $40,50,60,70,80,90$ e $99 \%$ de ocorrências na área de estudo entre 19171996, com CAD: 50mm, com valores menores ou iguais, referentes aos dados abaixo.

\begin{tabular}{c|ccccccccccccc} 
& \multicolumn{10}{c}{$\mathbf{3}^{\mathbf{0}}$ Decêndio } \\
\cline { 2 - 13 } PROB (\%) & JAN & FEV & MAR & ABR & MAI & JUN & JI & AGO & SET & OUT & NOV & DEZ \\
\hline $\mathbf{1 0}$ & $\mathbf{3 8}$ & 25 & 31 & 18 & 13 & 11 & 11 & 17 & 20 & 29 & 30 & 36 \\
$\mathbf{2 0}$ & 39 & 27 & 33 & 20 & 15 & 12 & 14 & 19 & 23 & 31 & 32 & 38 \\
$\mathbf{3 0}$ & 41 & 29 & 34 & 22 & 16 & 13 & 15 & 20 & 24 & 32 & 33 & 40 \\
$\mathbf{4 0}$ & 43 & 30 & 36 & 23 & 17 & 14 & 16 & 21 & 25 & 33 & 34 & 41 \\
$\mathbf{5 0}$ & 44 & 31 & 37 & 24 & 17 & 15 & 17 & 22 & 26 & 34 & 35 & 42 \\
$\mathbf{6 0}$ & 45 & 32 & 38 & 25 & 19 & 16 & 18 & 23 & 27 & 35 & 37 & 44 \\
$\mathbf{7 0}$ & 46 & 33 & 39 & 26 & 20 & 16 & 19 & 26 & 28 & 37 & 38 & 45 \\
$\mathbf{8 0}$ & 49 & 35 & 41 & 28 & 23 & 17 & 20 & 28 & 30 & 41 & 40 & 46 \\
$\mathbf{9 0}$ & 52 & 36 & 42 & 30 & 23 & 18 & 21 & 29 & 32 & 43 & 43 & 49 \\
$\mathbf{9 9 9}$ & 58 & 40 & 61 & 43 & 27 & 21 & 25 & 36 & 41 & 46 & 47 & 52 \\
\hline MEDIA & 45 & 32 & 39 & 26 & 19 & 15 & 17 & 24 & 28 & 36 & 37 & 43 \\
MEDIANA & 44 & 32 & 37 & 24 & 18 & 16 & 17 & 23 & 27 & 35 & 36 & 43 \\
\hline
\end{tabular}

Tabela 15-Probabilidades (\%) da evapotranspiração potencial decendial, com 10, 20, 30, $40,50,60,70,80,90$ e $99 \%$ de ocorrências na área de estudo entre 19171996, com CAD: $100 \mathrm{~mm}$, com valores menores ou iguais, referentes aos dados abaixo.

\begin{tabular}{|c|c|c|c|c|c|c|c|c|c|c|c|c|}
\hline \multirow{4}{*}{ PROB (\%) } & \multicolumn{12}{|c|}{ Evapotranspiraçāo potencial (mm) - CAD: $100 \mathrm{~mm}$} \\
\hline & \multicolumn{12}{|c|}{ Meses } \\
\hline & JAN & FEV & MAR & ABR & MAI & JUN & JUL & AGO & SET & OUT & NoV & DEZ \\
\hline & \multicolumn{12}{|c|}{$1^{\circ}$ Decêndio } \\
\hline 10 & 33 & 34 & 34 & 24 & 15 & 10 & 10 & 13 & 17 & 23 & 28 & 32 \\
\hline 20 & 36 & 35 & 35 & 26 & 17 & 12 & 12 & 14 & 19 & 24 & 29 & 34 \\
\hline 30 & 38 & 37 & 35 & 28 & 19 & 13 & 13 & 16 & 20 & 26 & 31 & 35 \\
\hline 40 & 39 & 38 & 37 & 28 & 20 & 14 & 14 & 17 & 21 & 27 & 32 & 37 \\
\hline so & 41 & 39 & 38 & 30 & 21 & 14 & 14 & 18 & 22 & 29 & 34 & 38 \\
\hline 60 & 41 & 40 & 39 & 31 & 21 & 16 & 15 & 18 & 23 & 31 & 35 & 39 \\
\hline 70 & 43 & 42 & 40 & 32 & 23 & 17 & 16 & 19 & 24 & 32 & 37 & 42 \\
\hline 80 & 47 & 45 & 41 & 34 & 24 & 18 & 17 & 21 & 26 & 35 & 39 & 44 \\
\hline 90 & 48 & 48 & 43 & 37 & 27 & 20 & 19 & 22 & 29 & 38 & 42 & 46 \\
\hline 99,9 & 51 & 51 & 47 & 42 & 32 & 26 & 21 & 48 & 36 & 48 & 51 & 55 \\
\hline MEDIA & 42 & 41 & 39 & 31 & 22 & 16 & 15 & 21 & 24 & 31 & 36 & 40 \\
\hline MEDLANA & 41 & 39 & 39 & 30 & 21 & 15 & 15 & 18 & 22 & 30 & 35 & 39 \\
\hline
\end{tabular}


Continuação:

Tabela 15-Probabilidades (\%) da evapotranspiração potencial decendial, com 10, 20,30, $40,50,60,70,80,90$ e $99 \%$ de ocorrências na área de estudo entre 19171996, com CAD: 100mm, com valores menores ou iguais, referentes aos dados abaixo.

\begin{tabular}{|c|c|c|c|c|c|c|c|c|c|c|c|c|}
\hline \multirow[t]{2}{*}{ PROB (\%) } & JAN & FEV & MAR & $\mathbf{A B R}$ & MAI & JUN & JUL & AGO & SET & OUT & NoV & DEZ \\
\hline & \multicolumn{12}{|c|}{$2^{\circ}$ Decêndio } \\
\hline 10 & 34 & 35 & 31 & 22 & 14 & 11 & 8 & 13 & 18 & 23 & 30 & 33 \\
\hline 20 & 36 & 37 & 32 & 23 & 16 & 12 & 11 & 16 & 20 & 25 & 32 & 36 \\
\hline 30 & 37 & 37 & 33 & 25 & 18 & 13 & 12 & 18 & 22 & 27 & 34 & 37 \\
\hline 40 & 39 & 38 & 35 & 25 & 18 & 14 & 13 & 18 & 23 & 28 & 34 & 38 \\
\hline 50 & 40 & 40 & 36 & 27 & 19 & 15 & 14 & 19 & 24 & 30 & 36 & 39 \\
\hline 60 & 41 & 42 & 37 & 28 & 20 & 16 & 15 & 20 & 26 & 31 & 36 & 40 \\
\hline 70 & 42 & 43 & 38 & 29 & 21 & 17 & 16 & 21 & 27 & 33 & 38 & 41 \\
\hline 90 & 44 & 45 & 40 & 31 & 23 & 18 & 18 & 23 & 30 & 35 & 39 & 44 \\
\hline 90 & 49 & 48 & 41 & 32 & 25 & 19 & 19 & 24 & 32 & 37 & 41 & 45 \\
\hline 99,9 & 54 & 50 & 45 & 38 & 27 & 21 & 23 & 27 & 38 & 47 & 52 & 50 \\
\hline MEDLA & 42 & 41 & 37 & 28 & 20 & 16 & 15 & 20 & 26 & 31 & 37 & 40 \\
\hline MEDIANA & 41 & 41 & 37 & 27 & 20 & 15 & 14 & 19 & 25 & 30 & 36 & 40 \\
\hline PROR (\%) & \multicolumn{12}{|c|}{$3^{\circ}$ Decêndio } \\
\hline 10 & 38 & 25 & 31 & 18 & 13 & 11 & 11 & 17 & 20 & 29 & 30 & 36 \\
\hline 20 & 39 & 28 & 33 & 20 & 15 & 12 & 14 & 19 & 23 & 31 & 32 & 39 \\
\hline 30 & 42 & 29 & 35 & 22 & 16 & 13 & 15 & 20 & 24 & 32 & 33 & 40 \\
\hline 40 & 43 & 30 & 36 & 23 & 17 & 15 & 16 & 21 & 25 & 33 & 34 & 41 \\
\hline 50 & 44 & 31 & 37 & 24 & 17 & 15 & 17 & 22 & 26 & 34 & 36 & 43 \\
\hline 60 & 45 & 33 & 38 & 25 & 19 & 16 & 18 & 24 & 28 & 36 & 37 & 44 \\
\hline 70 & 46 & 34 & 40 & 27 & 20 & 16 & 19 & 26 & 29 & 38 & 39 & 45 \\
\hline 80 & 49 & 35 & 41 & 29 & 23 & 17 & 20 & 28 & 30 & 41 & 40 & 46 \\
\hline 90 & 53 & 36 & 43 & 30 & 24 & 19 & 21 & 30 & 32 & 43 & 43 & 49 \\
\hline 99,9 & 58 & 40 & 61 & 43 & 28 & 21 & 25 & 37 & 41 & 47 & 47 & 53 \\
\hline MEDIA & 46 & 32 & 39 & 26 & 19 & 16 & 18 & 24 & 28 & 36 & 37 & 44 \\
\hline MEDIANA & 45 & 32 & 38 & 24 & 18 & 16 & 17 & 23 & 27 & 35 & 37 & 43 \\
\hline
\end{tabular}


Tabela 16- Probabilidades (\%) da evapotranspiração potencial decendial, com 10, 20, 30, $40,50,60,70,80,90$ e $99 \%$ de ocorrências na área de estudo entre 19171996, com CAD: $150 \mathrm{~mm}$, com valores menores ou iguais, referentes aos dados abaixo.

\begin{tabular}{|c|c|c|c|c|c|c|c|c|c|c|c|c|}
\hline \multirow{3}{*}{ PROB (\%) } & \multicolumn{11}{|c|}{ Evapotranspirą̧̄̃o potencial (mm)-CAD: $150 \mathrm{~mm}$} & \multirow[b]{2}{*}{ DER } \\
\hline & JAN & FEV & MAR & ABR & MAI & JUN & JUL & AGO & SET & OUT & NOV & \\
\hline & \multicolumn{11}{|c|}{$1^{\circ}$ Decêndio } & \\
\hline 10 & 33 & 34 & 34 & 24 & 15 & 10 & 10 & 13 & 17 & 23 & 28 & 32 \\
\hline 20 & 36 & 33 & 35 & 26 & 17 & 12 & 12 & 14 & 19 & 24 & 29 & 34 \\
\hline 30 & 38 & 37 & 35 & 28 & 19 & 13 & 13 & 16 & 20 & 26 & 31 & 35 \\
\hline 40 & 39 & 38 & 37 & 28 & 20 & 14 & 14 & 17 & 21 & 27 & 32 & 37 \\
\hline 50 & 41 & 39 & 38 & 30 & 21 & 14 & 14 & 18 & 22 & 29 & 34 & 38 \\
\hline 60 & 41 & 40 & 39 & 31 & 21 & 16 & 15 & 18 & 23 & 31 & 35 & 39 \\
\hline 70 & 43 & 42 & 40 & 32 & 23 & 17 & 16 & 19 & 24 & 32 & 37 & 42 \\
\hline 80 & 47 & 45 & 41 & 34 & 24 & 18 & 17 & 21 & 26 & 35 & 39 & 44 \\
\hline 90 & 48 & 48 & 43 & 37 & 27 & 20 & 19 & 22 & 29 & 38 & 42 & 46 \\
\hline 99,9 & 51 & 51 & 47 & 42 & 32 & 26 & 21 & 48 & 36 & 48 & 51 & 59 \\
\hline MEDIA & 42 & 41 & 39 & 31 & 22 & 16 & 15 & 21 & 24 & 31 & 36 & 40 \\
\hline MEDIANA & 41 & 39 & 39 & 30 & 21 & 15 & 15 & 18 & 22 & 30 & 35 & 39 \\
\hline PROB (\%) & \multicolumn{11}{|c|}{$2^{\circ}$ Decêndio } & \\
\hline 10 & 34 & 35 & 31 & 22 & 14 & 11 & 8 & 13 & 18 & 23 & 30 & 33 \\
\hline 20 & 36 & 36 & 32 & 23 & 16 & 12 & 11 & 16 & 20 & 25 & 32 & 35 \\
\hline 30 & 37 & 37 & 33 & 25 & 18 & 13 & 12 & 18 & 22 & 27 & 34 & 37 \\
\hline 40 & 39 & 38 & 35 & 25 & 18 & 14 & 13 & 18 & 23 & 28 & 34 & 38 \\
\hline 50 & 40 & 40 & 36 & 27 & 19 & 15 & 14 & 19 & 24 & 30 & 36 & 39 \\
\hline 60 & 41 & 42 & 37 & 28 & 20 & 16 & 15 & 20 & 26 & 31 & 36 & 40 \\
\hline 70 & 42 & 43 & 38 & 29 & 21 & 17 & 16 & 21 & 27 & 33 & 38 & 41 \\
\hline 80 & 44 & 45 & 39 & 30 & 23 & 18 & 18 & 22 & 29 & 35 & 39 & 43 \\
\hline 90 & 49 & 48 & 41 & 32 & 25 & 19 & 19 & 24 & 32 & 37 & 41 & 45 \\
\hline 99,9 & 54 & 50 & 45 & 38 & 27 & 21 & 23 & 27 & 38 & 47 & 52 & 50 \\
\hline MEDLA & 42 & 41 & 37 & 28 & 20 & 16 & 15 & 20 & 26 & 31 & 37 & 40 \\
\hline MEDLANA & 41 & 41 & 37 & 27 & 20 & 15 & 14 & 19 & 25 & 30 & 36 & 40 \\
\hline PROB (\%) & \multicolumn{11}{|c|}{$3^{\circ}$ Decêndio } & \\
\hline 10 & 38 & 25 & 31 & 18 & 13 & 11 & 11 & 17 & 20 & 29 & 30 & 36 \\
\hline 20 & 39 & 27 & 33 & 20 & 15 & 12 & 14 & 19 & 23 & 31 & 32 & 38 \\
\hline 30 & 42 & 29 & 35 & 22 & 16 & 13 & 15 & 20 & 24 & 32 & 33 & 40 \\
\hline 40 & 43 & 30 & 36 & 23 & 17 & 15 & 16 & 21 & 25 & 33 & 34 & 41 \\
\hline 50 & 44 & 31 & 37 & 24 & 17 & 15 & 17 & 22 & 26 & 34 & 36 & 43 \\
\hline 60 & 45 & 33 & 38 & 25 & 19 & 16 & 18 & 24 & 28 & 36 & 37 & 44 \\
\hline 70 & 46 & 34 & 40 & 27 & 20 & 16 & 19 & 26 & 29 & 38 & 39 & 45 \\
\hline 80 & 49 & 35 & 41 & 28 & 23 & 17 & 20 & 28 & 30 & 41 & 40 & 46 \\
\hline 90 & 53 & 36 & 43 & 30 & 24 & 19 & 21 & 30 & 32 & 43 & 43 & 49 \\
\hline 99,9 & 58 & 40 & 61 & 43 & 28 & 21 & 25 & 37 & 41 & 47 & 47 & 53 \\
\hline MEDIA & 46 & 32 & 39 & 26 & 19 & 16 & 18 & 24 & 28 & 36 & 37 & 44 \\
\hline MEDIANA & 45 & 32 & 38 & 24 & 18 & 16 & 17 & 23 & 27 & 35 & 37 & 43 \\
\hline
\end{tabular}


A evapotranspiração real na escala mensal, apresentada na Tabela 17, para as três CAD, mostra que, para $20 \%$ de probabilidades na estação chuvosa, ocorreram valores abaixo de $116 \mathrm{~mm}$ e para $80 \%$ abaixo de $132 \mathrm{~mm}$. Na estação seca, os valores abaixo de $50 \mathrm{~mm}$ apresentaram $20 \%$ de probabilidades e valores abaixo de $83 \mathrm{~mm}$ para $80 \%$. A média e mediana apresentaram valores próximos, diferindo em $2 \mathrm{~mm}$.

Tabela 17- Probabilidades (\%) da evapotranspiração real mensal, com 10, 20, 30, 40, 50, $60,70,80,90$ e $99 \%$ de ocorrências na área de estudo entre 1917-1996, com CAD: 50,100 e $150 \mathrm{~mm}$, com valores menores ou iguais, referentes aos dados abaixo.

\begin{tabular}{|c|c|c|c|c|c|c|c|c|c|c|c|c|}
\hline \multirow[b]{3}{*}{ PROB (\%) } & \multicolumn{11}{|c|}{ Evapotranspirap̧āo real mensal (mm) - CAD: $50 \mathrm{~mm}$} & \multirow[b]{3}{*}{ DEZ } \\
\hline & \multicolumn{10}{|c|}{ Meses } & \multirow[b]{2}{*}{ Nov } & \\
\hline & JAN & FEV & MAR & ABR & MAI & JUN & JUL & AGO & SET & OUT & & \\
\hline 10 & 109 & 89 & 74 & 38 & 16 & 15 & 4 & 14 & 11 & 60 & 58 & 106 \\
\hline 20 & 116 & 99 & 87 & 50 & 27 & 22 & 16 & 15 & 25 & 69 & 84 & 109 \\
\hline 30 & 120 & 103 & 98 & 56 & 36 & 28 & 21 & 20 & 33 & 77 & 96 & 114 \\
\hline 40 & 122 & 105 & 104 & 64 & 44 & 34 & 27 & 27 & 47 & 83 & 100 & 117 \\
\hline 50 & 125 & 108 & 107 & 70 & 47 & 36 & 32 & 29 & 57 & 90 & 102 & 120 \\
\hline 60 & 127 & 111 & 111 & 73 & 51 & 39 & 34 & 34 & 61 & 92 & 106 & 122 \\
\hline 70 & 129 & 113 & 115 & 76 & 54 & 44 & 40 & 46 & 65 & 94 & 109 & 126 \\
\hline 80 & 132 & 117 & 117 & 83 & 57 & 46 & 42 & 53 & 67 & 96 & 112 & 128 \\
\hline 90 & 138 & 120 & 122 & 86 & 62 & 48 & 44 & 58 & 75 & 103 & 120 & 132 \\
\hline 99,9 & 150 & 135 & 133 & 94 & 75 & 57 & 61 & 102 & 85 & 130 & 135 & 156 \\
\hline MEDLA & 127 & 110 & 107 & 69 & 47 & 37 & 32 & 40 & 53 & 89 & 102 & 123 \\
\hline MEDIANA & 126 & 110 & 109 & 71 & 49 & 38 & 33 & 32 & 59 & 91 & 104 & 121 \\
\hline PROB (\%) & & & & & AD: 10 & & & & & & & \\
\hline 10 & 111 & 97 & 87 & 47 & 25 & 23 & 12 & 21 & 21 & 63 & 65 & 106 \\
\hline 20 & 119 & 100 & 92 & 59 & 37 & 27 & 23 & 27 & 37 & 73 & 88 & 109 \\
\hline 30 & 120 & 104 & 101 & 64 & 42 & 32 & 27 & 30 & 40 & 80 & 97 & 114 \\
\hline 40 & 122 & 106 & 105 & 68 & 47 & 35 & 31 & 33 & so & 85 & 100 & 117 \\
\hline 50 & 125 & 108 & 108 & 72 & 50 & 38 & 34 & 37 & 58 & 90 & 103 & 120 \\
\hline 60 & 127 & 111 & 111 & 76 & 53 & 41 & 39 & 41 & 63 & 92 & 106 & 122 \\
\hline 70 & 131 & 113 & 115 & 81 & 55 & 44 & 41 & 48 & 66 & 95 & 109 & 126 \\
\hline 80 & 132 & 117 & 117 & 84 & 59 & 47 & 44 & 53 & 67 & 96 & 113 & 128 \\
\hline 90 & 138 & 121 & 122 & 88 & 63 & 49 & 46 & 58 & 76 & 105 & 120 & 133 \\
\hline 99,9 & 150 & 135 & 133 & 94 & 75 & 57 & 62 & 102 & 86 & 130 & 136 & 156 \\
\hline MEDIA & 127 & 111 & 109 & 73 & 51 & 39 & 36 & 45 & 56 & 91 & 104 & 123 \\
\hline MEDIANA & 126 & 110 & 110 & 74 & 51 & 40 & 37 & 39 & 60 & 91 & 104 & 121 \\
\hline
\end{tabular}


Continuação:

Tabela 17- Probabilidades (\%) da evapotranspiração real mensal, com 10, 20, 30, 40, 50, $60,70,80,90$ e 99\% de ocorrências na área de estudo entre 1917-1996, com CAD: 50,100 e $150 \mathrm{~mm}$, com valores menores ou iguais, referentes aos dados abaixo.

\begin{tabular}{c|ccccccccccccc} 
& \multicolumn{10}{c}{ CAD:150 mm } \\
\cline { 2 - 14 } PROB (\%) & JAN & FEV & MAR & ABR & MAI & JUN & JUL & AGO & SET & OUT & NOV & DEZ \\
\hline 10 & 111 & 97 & 92 & 52 & 33 & 26 & 18 & 26 & 30 & 66 & 73 & 106 \\
20 & 119 & 100 & 97 & 63 & 41 & 31 & 27 & 34 & 40 & 77 & 91 & 109 \\
30 & 120 & 105 & 101 & 67 & 46 & 35 & 30 & 36 & 50 & 83 & 98 & 114 \\
40 & 122 & 107 & 105 & 70 & 50 & 37 & 34 & 39 & 54 & 87 & 100 & 117 \\
50 & 126 & 109 & 108 & 73 & 52 & 39 & 36 & 41 & 60 & 91 & 102 & 120 \\
60 & 128 & 112 & 111 & 78 & 54 & 42 & 40 & 46 & 64 & 92 & 106 & 122 \\
70 & 131 & 114 & 115 & 82 & 56 & 45 & 42 & 51 & 67 & 95 & 109 & 126 \\
80 & 132 & 117 & 117 & 84 & 59 & 48 & 46 & 54 & 68 & 97 & 113 & 128 \\
90 & 138 & 121 & 122 & 91 & 63 & 50 & 49 & 58 & 76 & 105 & 120 & 133 \\
99,9 & 150 & 135 & 133 & 94 & 81 & 57 & 62 & 102 & 87 & 130 & 136 & 156 \\
\hline MEDIA & 128 & 112 & 110 & 75 & 53 & 41 & 39 & 49 & 60 & 92 & 105 & 123 \\
MEDIANA & 127 & 111 & 110 & 75 & 53 & 41 & 38 & 43 & 62 & 91 & 104 & 121 \\
\hline
\end{tabular}

A evapotranspiração real para a CAD: 50,100 e $150 \mathrm{~mm}$, no $1^{\circ}$ decêndio, (Tabelas 18,19 e 20) apresentou, para $20 \%$ de probabilidades, valores menores que 36 mm e para $80 \%$, valores abaixo de $44 \mathrm{~mm}$ na estação chuvosa. Na estação seca, os valores foram abaixo de $23 \mathrm{~mm}$ para $20 \%$ e menores que $32 \mathrm{~mm}$ para $80 \%$ de probabilidades. No $2^{\circ}$ decêndio para $20 \%$ de probabilidades, os valores foram abaixo de $35 \mathrm{~mm}$ e para $80 \%$, os valores foram abaixo de $42 \mathrm{~mm}$ na estação chuvosa. Na estação seca, ocorreram em $20 \%$ de probabilidades, valores abaixo de $18 \mathrm{~mm}$ e $28 \mathrm{~mm}$ para $80 \%$. Para o $3^{\circ}$ decêndio, em $20 \%$ de probabilidades ocorreram valores abaixo de $38 \mathrm{~mm}$ e valores menores que $47 \mathrm{~mm}$ para $80 \%$ de probabilidades na estação chuvosa e valores menores que $14 \mathrm{~mm}$ para $20 \%$ de probabilidades e $25 \mathrm{~mm}$ para $80 \%$ de probabilidades na estação seca. 
Tabela 18- Probabilidades (\%) da evapotranspiração real decendial, com 10, 20, 30, 40, $50,60,70,80,90$ e 99\% de ocorrências na área de estudo entre 1917-1996, com CAD: $50 \mathrm{~mm}$, com valores menores ou iguais, referentes aos dados abaixo.

\begin{tabular}{|c|c|c|c|c|c|c|c|c|c|c|c|c|}
\hline \multirow{3}{*}{ PROB (\%) } & \multicolumn{12}{|c|}{ Evapotranspưração real decendial (mm) - CAD: $50 \mathrm{~mm}$} \\
\hline & JAN & FEV & MAR & ABR & MAI & JUN & JUL & $\mathbf{A G O}$ & SET & OUT & NOV & DEZ \\
\hline & \multicolumn{12}{|c|}{$1^{\circ}$ Decêndio } \\
\hline 10 & 30 & 29 & 23 & 12 & 3 & 1 & 3 & 3 & 1 & 4 & 14 & 24 \\
\hline 20 & 34 & 33 & 28 & 18 & 7 & 4 & 4 & 4 & 3 & 11 & 23 & 32 \\
\hline 30 & 36 & 34 & 31 & 22 & 9 & 7 & 7 & 5 & 5 & 18 & 27 & 33 \\
\hline 40 & 37 & 36 & 33 & 24 & 12 & 9 & 8 & 7 & 9 & 22 & 29 & 34 \\
\hline 50 & 38 & 37 . & 35 & 25 & 15 & 10 & 9 & 9 & 11 & 24 & 31 & 35 \\
\hline 60 & 40 & 38 & 36 & 26 & 17 & 13 & 10 & 11 & 16 & 25 & 32 & 36 \\
\hline 70 & 41 & 39 & 38 & 28 & 19 & 13 & 11 & 12 & 17 & 26 & 34 & 38 \\
\hline $\mathbf{8 0}$ & 42 & 41 & 39 & 30 & 22 & 14 & 13 & 14 & 19 & 28 & 37 & 41 \\
\hline 90 & 44 & 44 & 41 & 32 & 23 & 16 & 15 & 16 & 20 & 30 & 40 & 43 \\
\hline 99,9 & 48 & 49 & 42 & 36 & 27 & 20 & 19 & 25 & 28 & 35 & 51 & 51 \\
\hline MEDIA & 39 & 38 & 34 & 25 & 15 & 11 & 10 & 11 & 13 & 22 & 32 & 37 \\
\hline MEDIANA & 39 & 38 & 35 & 26 & 16 & 11 & 10 & 10 & 13 & 24 & 31 & 36 \\
\hline PROB (\%) & \multicolumn{12}{|c|}{$2^{\circ}$ Decêndio } \\
\hline 10 & 31 & 30 & 20 & 7 & 2 & 1 & 1 & 2 & 2 & 13 & 14 & 30 \\
\hline 20 & 34 & 35 & 28 & 12 & 7 & 4 & 2 & 4 & 4 & 19 & 24 & 34 \\
\hline 30 & 36 & 36 & 31 & 16 & 10 & 7 & 5 & 5 & 9 & 23 & 28 & 35 \\
\hline 40 & 37 & 37 & 31 & 19 & 13 & 9 & 7 & 6 & 12 & 25 & 30 & 36 \\
\hline 50 & 38 & 37 & 32 & 20 & 15 & 10 & 8 & 7 & 15 & 27 & 33 & 38 \\
\hline 60 & 39 & 38 & 35 & 22 & 17 & 11 & 9 & 9 & 18 & 28 & 34 & 38 \\
\hline 70 & 40 & 40 & 36 & 24 & 19 & 12 & 10 & 11 & 20 & 30 & 36 & 40 \\
\hline 80 & 42 & 41 & 37 & 26 & 20 & 15 & 12 & 13 & 22 & 31 & 38 & 41 \\
\hline 90 & 44 & 42 & 39 & 29 & 23 & 16 & 15 & 18 & 24 & 33 & 39 & 44 \\
\hline 99,9 & 49 & 49 & 43 & 32 & 27 & 19 & 18 & 24 & 29 & 40 & 43 & 49 \\
\hline MEDIA & 39 & 39 & 33 & 21 & 15 & 11 & 9 & 10 & 15 & 27 & 32 & 39 \\
\hline MEDIANA & 38 & 38 & 34 & 21 & 16 & 11 & 8 & 8 & 16 & 27 & 33 & 38 \\
\hline PROB (\%) & \multicolumn{12}{|c|}{$3^{0}$ Decêndio } \\
\hline 10 & 36 & 17 & 15 & 4 & 2 & 1 & 2 & 3 & 3 & 11 & 18 & 32 \\
\hline 20 & 38 & 25 & 22 & 8 & 5 & 5 & 4 & 4 & 8 & 23 & 26 & 36 \\
\hline 30 & 39 & 27 & 27 & 11 & 6 & 7 & 6 & 5 & 13 & 27 & 30 & 38 \\
\hline 40 & 41 & 28 & 29 & 13 & 8 & 9 & 7 & 7 & 18 & 29 & 31 & 38 \\
\hline 50 & 42 & 29 & 33 & 16 & 11 & 10 & 8 & 11 & 20 & 31 & 33 & 40 \\
\hline 60 & 44 & 30 & 36 & 18 & 13 & 12 & 10 & 14 & 22 & 32 & 35 & 42 \\
\hline 70 & 45 & 31 & 37 & 20 & 15 & 12 & 11 & 17 & 23 & 34 & 36 & 43 \\
\hline 80 & 46 & 33 & 39 & 22 & 16 & 13 & 12 & 19 & 25 & 35 & 39 & 45 \\
\hline 90 & 48 & 34 & 40 & 25 & 20 & 15 & 14 & 21 & 26 & 37 & 42 & 47 \\
\hline 99,9 & 56 & 37 & 45 & 33 & 25 & 19 & 23 & 36 & 32 & 44 & 47 & 52 \\
\hline MEDIA & 43 & 29 & 32 & 17 & 12 & 10 & 10 & 14 & 19 & 30 & 34 & 41 \\
\hline MEDIANA & 43 & 29 & 34 & 17. & 12 & 11 & 9 & 12 & 21 & 31 & 34 & 41 \\
\hline
\end{tabular}


Tabela 19- Probabilidades (\%) da evapotranspiração real decendial, com 10, 20, 30, 40, $50,60,70,80,90$ e $99 \%$ de ocorrências para a CAD: $100 \mathrm{~mm}$, com valores menores ou iguais, referentes aos dados abaixo.

\begin{tabular}{|c|c|c|c|c|c|c|c|c|c|c|c|c|}
\hline \multirow{3}{*}{ PROB (\%) } & \multicolumn{11}{|c|}{ Evapotranspiraçao real (mm)-CADi $100 \mathrm{~mm}$} & \multirow[b]{2}{*}{ DEZ } \\
\hline & JAN & FEV & MAR & ABR & MAI & JUN & JUL & AGO & SET & OUT & NOV & \\
\hline & \multicolumn{12}{|c|}{$1^{\circ}$ Decêndio } \\
\hline 10 & 32 & 32 & 28 & 18 & 7 & 4 & 4 & 5 & 5 & 8 & 17 & 27 \\
\hline 20 & 36 & 34 & 31 & 22 & 11 & 6 & 7 & 7 & 7 & 14 & 22 & 32 \\
\hline 30 & 36 & 36 & 33 & 24 & 12 & 9 & 8 & 9 & 10 & 21 & 27 & 33 \\
\hline 40 & 38 & 37 & 34 & 25 & 15 & 10 & 9 & 10 & 12 & 23 & 29 & 34 \\
\hline 50 & 39 & 38 & 35 & 26 & 16 & 12 & 10 & 11 & 13 & 25 & 31 & 36 \\
\hline 60 & 41 & 39 & 37 & 28 & 18 & 13 & 11 & 12 & 16 & 26 & 32 & 37 \\
\hline 70 & 42 & 40 & 38 & 29 & 20 & 14 & 13 & 13 & 18 & 26 & 35 & 39 \\
\hline 80 & 43 & 42 & 39 & 32. & 22 & 15 & 13 & 16 & 19 & 28 & 37 & 42 \\
\hline 90 & 46 & 45 & 41 & 33 & 24 & 17 & 15 & 17 & 21 & 30 & 39 & 43 \\
\hline 99,9 & 48 & 49 & 44 & 36 & 27 & 20 & 19 & 25 & 28 & 35 & 51 & 51 \\
\hline MEDIA & 40 & 39 & 36 & 27 & 17 & 12 & 11 & 12 & 15 & 24 & 32 & 37 \\
\hline MEDIANA & 40 & 39 & 36 & 27 & 17 & 12 & 11 & 11 & 14 & 25 & 32 & 36 \\
\hline PROB (\%) & \multicolumn{12}{|c|}{$2^{\circ}$ Decêudio } \\
\hline 10 & 32 & 42 & 25 & 11 & 6 & 4 & 2 & 4 & 6 & 14 & 18 & 32 \\
\hline 20 & 35 & 38 & 30 & 15 & 9 & 6 & 5 & 7 & 9 & 19 & 26 & 34 \\
\hline 30 & 37 & 43 & 31 & 19 & 12 & 9 & 7 & 8 & 11 & 24 & 30 & 36 \\
\hline 40 & 37 & 37 & 32 & 22 & 14 & 10 & 8 & 9 & 14 & 25 & 31 & 36 \\
\hline 50 & 39 & 31 & 34 & 22 & 15 & 11 & 10 & 10 & 17 & 27 & 32 & 38 \\
\hline 60 & 40 & 36 & 36 & 23 & 18 & 12 & 10 & 12 & 18 & 29 & 34 & 39 \\
\hline 70 & 41 & 38 & 37 & 25 & 19 & 13 & 11 & 13 & 20 & 30 & 36 & 40 \\
\hline 80 & 42 & 40 & 38 & 28 & 21 & 15 & 12 & 15 & 22 & 32 & 38. & 41 \\
\hline 90 & 45 & 42 & 39 & 30 & 23 & 16 & 15 & 19 & 24 & 34 & 39 & 44 \\
\hline 99,9 & 49 & 49 & 43 & 34 & 27 & 19 & 18 & 24 & 30 & 40 & 45 & 49 \\
\hline MEDIA & 40 & 39 & 34 & 23 & 16 & 12 & 10 & 12 & 17 & 27 & 33 & 39 \\
\hline MEDIANA & 40 & 39 & 35 & 23 & 17 & 12 & 10 & 11 & 17 & 28 & 33 & 38 \\
\hline PROB (\%) & \multicolumn{12}{|c|}{$3^{\circ}$ Decêndio } \\
\hline 10 & 37 & 23 & 21 & 9 & 4 & 3 & 3 & 5 & 7 & 16 & 22 & 35 \\
\hline 20 & 39 & 25 & 27 & 12 & 8 & 7 & 7 & 8 & 10 & 24 & 28 & 38 \\
\hline 30 & 41 & 28 & 29 & 14 & 9 & 9 & 8 & 10 & 15 & 27 & 30 & 38 \\
\hline 40 & 42 & 29 & 32 & 17 & 11 & 10 & 10 & 11 & 18 & 29 & 32 & 40 \\
\hline 50 & 44 & 30 & 35 & 19 & 12 & 11 & 10 & 13 & 20 & 31 & 33 & 41 \\
\hline 60 & 45 & 31 & 36 & 20 & 14 & 12 & 11 & 15. & 23 & 32 & 35 & 43 \\
\hline 70 & 46 & 32 & 37 & 22 & 15 & 13 & 12 & 17 & 23 & 35 & 36 & 44 \\
\hline 80 & 47 & 33 & 39 & 23 & 17 & 15 & 13 & 19 & 25 & 36 & 40 & 46 \\
\hline 90 & 49 & 35 & 41 & 25 & 20 & 15 & 16 & 23 & 27 & 38 & 42 & 48 \\
\hline 99,9 & 56 & 37 & 52 & 33 & 25 & 19 & 24 & 37 & 32 & 45 & 47 & 53 \\
\hline MEDIA & 45 & 30 & 35 & 19 & 14 & 11 & 11 & 16 & 20 & 31 & 34 & 42 \\
\hline MEDLANA & 44 & 30 & 36 & 20 & 13 & 11 & 11 & 14 & 21 & 32 & 34 & 42 \\
\hline
\end{tabular}


Tabela 20- Probabilidades (\%) da evapotranspiração real decendial, com 10, 20, 30, 40, $50,60,70,80,90$ e $99 \%$ de ocorrências para a CAD: $150 \mathrm{~mm}$, com valores menores ou iguais, referentes aos dados abaixo.

\begin{tabular}{|c|c|c|c|c|c|c|c|c|c|c|c|c|}
\hline \multirow{3}{*}{$\operatorname{PROB}(\%)$} & \multicolumn{11}{|c|}{ Evapotranspiração real decendial (mm) - CAD: 150 mm } & \multirow[b]{2}{*}{ DEZ } \\
\hline & JAN & FEV & MAR & ABR & MAI & IUN & JUL & AGO & SET & out & NoV & \\
\hline & \multicolumn{11}{|c|}{$1^{2}$ Decêndio } & \\
\hline 10 & 33 & 33 & 30 & 19 & 10 & 5 & 6 & 7 & 7 & 11 & 17 & 29 \\
\hline 20 & 36 & 34 & 33 & 23 & 12 & 8 & 8 & 8 & 9 & 17 & 23 & 32 \\
\hline 30 & 37 & 36 & 34 & 25 & 13 & 10 & 9 & 10 & 12 & 21 & 28 & 34 \\
\hline 40 & 38 & 37 & 35 & 26 & 15 & 11 & 10 & 11 & 13 & 23 & 29 & 35 \\
\hline 50 & 39 & 38 & 35 & 27 & 17 & 13 & 11 & 12 & 15 & 24 & 31 & 36 \\
\hline 60 & 41 & 39 & 37 & 28 & 18 & 13 & 12 & 13 & 17 & 26 & 33 & 37 \\
\hline 70 & 42 & 40 & 38 & 30 & 21 & 14 & 13 & 14 & 18 & 27 & 35 & 39 \\
\hline 80 & 44 & 43 & 40 & 32 & 22 & 16 & 14 & 16 & 19 & 29 & 37 & 42 \\
\hline 90 & 47 & 45 & 41 & 34 & 24 & 17 & 15 & 17 & 22 & 31 & 39 & 44 \\
\hline 99,9 & 49 & 49 & 44 & 37 & 27 & 21 & 19 & 25 & 28 & 35 & 51 & 51 \\
\hline MEDIA & 40 & 39 & 37 & 28 & 18 & 13 & 12 & 13 & 16 & 24 & 32 & 38 \\
\hline MEDIANA & 40 & 39 & 36 & 27 & 17 & 13 & 11 & 13 & 16 & 25 & 32 & 36 \\
\hline PROB (\%) & \multicolumn{12}{|c|}{$2^{\circ}$ Decêndio } \\
\hline 10 & 33 & 34 & 28 & 14 & 8 & 6 & 4 & 6 & 9 & 17 & 19 & 32 \\
\hline 20 & 35 & 35 & 31 & 18 & 10 & 7 & 6 & 8 & 11 & 20 & 28 & 35 \\
\hline 30 & 37 & 37 & 32 & 20 & 14 & 10 & 8 & 10 & 12 & 24 & 30 & 36 \\
\hline 40 & 38 & 38 & 33 & 22 & 15 & 11 & 9 & 11 & 15 & 26 & 31 & 37 \\
\hline 50 & 39 & 39 & 34 & 23 & 16 & 11 & 11 & 12 & 17 & 27 & 32 & 38 \\
\hline 60 & 40 & 40 & 36 & 24 & 18 & 13 & 12 & 14 & 19 & 29 & 34 & 39 \\
\hline 70 & 41 & 41 & 37 & 25 & 19 & 14 & 12 & 14 & 20 & 30 & 36 & 40 \\
\hline 80 & 43 & 42 & 38 & 28 & 21 & 15 & 13 & 15 & 22 & 31 & 38 & 42 \\
\hline 90 & 45 & 44 & 39 & 30 & 23 & 16 & 15 & 19 & 24 & 35 & 39 & 45 \\
\hline 99,9 & 49 & 49 & 43 & 35 & 27 & 19 & 20 & 24 & 30 & 40 & 47 & 49 \\
\hline MEDIA & 40 & 40 & 35 & 24 & 17 & 12 & 11 & 13 & 18 & 28 & 33 & 39 \\
\hline MEDLANA & 40 & 39 & 35 & 23 & 17 & 12 & 11 & 13 & 18 & 28 & 33 & 39 \\
\hline PROB (\%) & \multicolumn{12}{|c|}{$3^{\circ}$ Decêndio } \\
\hline 10 & 37 & 23 & 23 & 13 & 7 & 5 & 5 & 7 & 11 & 19 & 23 & 36 \\
\hline 20 & 39 & 26 & 28. & 14 & 9 & 8 & 8 & 10 & 13 & 25 & 28 & 38 \\
\hline 30 & 41 & 28 & 30 & 16 & 11 & 9 & 10 & 12 & 17 & 28 & 30 & 39 \\
\hline 40 & 43 & 29 & 33 & 18 & 12 & 11 & 10 & 14 & 19 & 30 & 32 & 40 \\
\hline 50 & 44 & 30 & 35 & 20 & 13 & 11 & 11 & 15 & 21 & 31 & 33 & 41 \\
\hline 60 & 45 & 31 & 37 & 22 & 15 & 12 & 12 & 16 & 22 & 32 & 35 & 43 \\
\hline 70 & 46 & 32 & 38 & 23 & 16 & 14 & 13 & 18 & 24 & 35 & 36 & 44 \\
\hline 80 & 47 & 34 & 39 & 24 & 17 & 15 & 15 & 19 & 25 & 36 & 40 & 46 \\
\hline 90 & 49 & 35 & 42 & 26 & 20 & 16 & 16 & 23 & 28 & 38 & 42 & 48 \\
\hline 99,9 & 56 & 37 & 54 & 33 & 25 & 19 & 24 & 37 & 32 & 45 & 47 & 53 \\
\hline MEDIA & 45 & 30 & 36 & 21 & 15 & 12 & 12 & 17 & 21 & 32 & 35 & 43 \\
\hline MEDIANA & 45 & 30 & 36 & 21 & 14 & 12 & 12 & 15 & 22 & 32 & 34 & 42 \\
\hline
\end{tabular}


A Tabela 21 abaixo, apresenta os valores do armazenamento hídrico mensal para as CAD: 50,100 e $150 \mathrm{~mm}$.

Tabela 21- Probabilidades (\%) do armazenamento hídrico mensal, com 10, 20,30,40, $50,60,70,80,90$ e $99 \%$ de ocorrências para as CAD: 50,100 e $150 \mathrm{~mm}$, com valores menores ou iguais, referentes aos dados abaixo

\begin{tabular}{|c|c|c|c|c|c|c|c|c|c|c|c|c|}
\hline \multirow[b]{2}{*}{ PROB (\%) } & \multicolumn{11}{|c|}{ Armazenamento hídrico mensal (mm) - CAD: $50 \mathrm{~mm}$} & \multirow[b]{2}{*}{ DEZ } \\
\hline & JAN & FEV & MAR & ABR & MAI & $\mathbf{J U N}$ & JUL & AGO & SET & OUT & Nov & \\
\hline 10 & 25 & 12 & 9 & 3 & 2 & 1 & 1 & 1 & 1 & 1 & 3 & 24 \\
\hline 20 & 50 & 34 & 15 & 8 & 4 & 4 & 3 & 2 & 1 & 3 & 9 & 41 \\
\hline 30 & 50 & 48 & 31 & 13 & 7 & 6 & 8 & 4 & 3 & 7 & 17 & 50 \\
\hline 40 & 50 & 50 & 50 & 16 & 13 & 15 & 13 & 5 & 5 & 19 & 32 & 50 \\
\hline 50 & 50 & 50 & 50 & 20 & 20 & 22 & 17 & 7 & 8 & 24 & 38 & 50 \\
\hline 60 & 50 & 50 & 50 & 27 & 30 & 30 & 19 & 10 & 12 & 34 & 50 & 50 \\
\hline 70 & 50 & 50 & 50 & 45 & 38 & 34 & 21 & 15 & 19 & 50 & 50 & 50 \\
\hline 80 & 50 & 50 & 50 & 50 & 50 & 47 & 29 & 19 & 47 & 50 & 50 & 50 \\
\hline 90 & 50 & 50 & 50 & 50 & 50 & 50 & 50 & 44 & 50 & 50 & 50 & 50 \\
\hline 99,9 & 30 & 50 & 50 & 50 & 50 & 50 & 50 & 30 & 50 & 50 & 50 & 30 \\
\hline MEDIA & 48 & 44 & 40 & 28 & 26 & 26 & 21 & 16 & 20 & 29 & 35 & 47 \\
\hline \multirow[t]{2}{*}{ MEDIANA } & 30 & 30 & 50 & 24 & 25 & 26 & 18 & 9 & 10 & 29 & 44 & 50 \\
\hline & \multicolumn{12}{|c|}{ CAD: $100 \mathrm{~mm}$} \\
\hline PROB (\%) & JAN & FEV & MAR & ABR & MAI & JUN & JUL & AGO & SET & OUT & Nov & DEZ \\
\hline 10 & 59 & 46 & 40 & 26 & 17 & 12 & 11 & 11 & 8 & 11 & 17 & 52 \\
\hline 20 & 92 & 80 & 54 & 40 & 28 & 27 & 22 & 19 & 14 & 18 & 28 & 73 \\
\hline 30 & 100 & 95 & 79 & 50 & 38 & 35 & 34 & 25 & 19 & 30 & 45 & 100 \\
\hline 40 & 100 & 100 & 97 & 56 & 48 & 55 & 42 & 30 & 27 & 39 & 58 & 100 \\
\hline 50 & 100 & 100 & 100 & 63 & 59 & 60 & 51 & 33 & 31 & 47 & 79 & 100 \\
\hline 60 & 100 & 100 & 100 & 73 & 73 & 67 & 55 & 38 & 38 & 68 & 91 & 100 \\
\hline 70 & 100 & 100 & 100 & 95 & 86 & 76 & 62 & 45 & 54 & 91 & 100 & 100 \\
\hline 80 & 100 & 100 & 100 & 100 & 100 & 89 & 72 & 60 & 76 & 100 & 100 & 100 \\
\hline 90 & 100 & 100 & 100 & 100 & 100 & 100 & 96 & 85 & 100 & 100 & 100 & 100 \\
\hline 99,9 & 100 & 100 & 100 & 100 & 100 & 100 & 100 & 100 & 100 & 100 & 100 & 100 \\
\hline MEDIA & 95 & 92 & 87 & 70 & 65 & 62 & 55 & 45 & 47 & 61 & 72 & 93 \\
\hline \multirow[t]{2}{*}{ MEDIANA } & 100 & 100 & 100 & 68 & 66 & 64 & 53 & 36 & 34 & 58 & 85 & 100 \\
\hline & \multicolumn{12}{|c|}{ CAD: $150 \mathrm{~mm}$} \\
\hline PROB (\%) & JAN & FEV & MAR & ABR & MAI & JUN & JLL & AGO & SET & OUT & MOV & DEZ \\
\hline 10 & 104 & 90 & 81 & 61 & 46 & 36 & 33 & 30 & 25 & 32 & 35 & 84 \\
\hline 20 & 136 & 128 & 99 & 81 & 64 & 62 & 52 & 45 & 38 & 45 & 57 & 102 \\
\hline 30 & 150 & 144 & 125 & 94 & 79 & 74 & 72 & 57 & 48 & 60 & 81 & 137 \\
\hline 40 & 150 & 150 & 143 & 102 & 91 & 92 & 80 & 63 & 57 & 67 & 96 & 150 \\
\hline 50 & 150 & 150 & 150 & 109 & 106 & 104 & 91 & 71 & 67 & 78 & 110 & 150 \\
\hline 60 & 150 & 150 & 150 & 120 & 122 & 113 & 99 & 76 & 76 & 110 & 132 & 150 \\
\hline 70 & 150 & 150 & 150 & 145 & 134 & 125 & 109 & 87 & 88 & 130 & 147 & 150 \\
\hline 80 & 150 & 150 & 150 & 150 & 150 & 138 & 120 & 104 & 111 & 144 & 150 & 150 \\
\hline 90 & 150 & 150 & 150 & 150 & 150 & 150 & 138 & 130 & 150 & 150 & 150 & 150 \\
\hline 99,9 & 150 & 150 & 150 & 150 & 150 & 150 & 150 & 150 & 150 & 150 & 150 & 150 \\
\hline MEDIA & 144 & 141 & 135 & 116 & 109 & 104 & 94 & 81 & 81 & 97 & 111 & 137 \\
\hline MEDIANA & 150 & 150 & 150 & 115 & 114 & 108 & 95 & 73 & 72 & 94 & 121 & 130 \\
\hline
\end{tabular}


Verificou-se que para a CAD de $50 \mathrm{~mm}$ ocorreram para $20 \%$ de probabilidade, valores menores que $50 \mathrm{~mm}$ e para $80 \%$, valores iguais a $50 \mathrm{~mm}$ na estação chuvosa; na estação seca, os valores foram menores que $8 \mathrm{~mm}$ para $20 \%$ e para $80 \%$ valores abaixo de $50 \mathrm{~mm}$. Para a CAD: $100 \mathrm{~mm}$ os valores abaixo de $92 \mathrm{~mm}$ tiveram $20 \%$ de probabilidade de ocorrência e valores iguais a $100 \mathrm{~mm}$ tiveram $80 \%$ de probabilidade na estação chuvosa; na estação seca, valores abaixo de $40 \mathrm{~mm}$ tiveram $20 \%$ de probabilidade e menores que $100 \mathrm{~mm}$ tiveram $80 \%$ de probabilidade. A CAD: $150 \mathrm{~mm}$ apresentou para $20 \%$ de probabilidade, valores abaixo de $136 \mathrm{~mm}$ e para $80 \%$, valores abaixo de $150 \mathrm{~mm}$, chegando a $144 \mathrm{~mm}$ na estação chuvosa. $\mathrm{Na}$ estação seca, para $20 \%$, os valores foram menores que $81 \mathrm{~mm}$ e para $80 \%$, abaixo de $150 \mathrm{~mm}$.

$\mathrm{Na}$ Tabela 22, estão representados os valores do armazenamento hídrico decendial para a CAD de $50 \mathrm{~mm}$. Verificou-se que, no $1^{\circ}, 2^{\circ}$ e $3^{\circ}$ decêndios ocorreu $20 \%$ de probabilidade para valores abaixo de $32 \mathrm{~mm}$ e $80 \%$ para valores iguais a $50 \mathrm{~mm}$ na estação chuvosa, enquanto que na estação seca, os valores foram menores que $14 \mathrm{~mm}$ em $20 \%$ de probabilidade e abaixo de $50 \mathrm{~mm}$ para $80 \%$ de probabilidade.

Tabela 22- Probabilidades (\%) do armazenamento hídrico decendial, com 10, 20, 30, 40, $50,60,70,80,90$ e $99 \%$ de ocorrências para a CAD: $50 \mathrm{~mm}$, com valores menores ou iguais, referentes aos dados abaixo.

\begin{tabular}{|c|c|c|c|c|c|c|c|c|c|c|c|c|}
\hline \multirow{3}{*}{ PROB (\%) } & \multicolumn{10}{|c|}{ Armazenamento hidrico (mm) - CAD: $50 \mathrm{~mm}$} & \multirow{3}{*}{ NOV } & \multirow[b]{2}{*}{ DEZ } \\
\hline & JAN & FEV & MAR & ABR & MAI & JUN & JUL & AGO & SET & OUT & & \\
\hline & \multicolumn{10}{|c|}{$1^{\circ}$ Decêndio } & & \\
\hline 10 & 20 & 19 & 13 & 5 & 4 & $3^{4}$ & 2 & 3 & 2 & 1 & 5 & 17 \\
\hline 20 & 29 & 31 & 23 & 14 & 6 & 5 & 9 & 8 & 4 & 3 & 12 & 23 \\
\hline 30 & 39 & 39 & 26 & 21 & 12 & 15 & 14 & 10 & 5 & 10 & 24 & 30 \\
\hline 40 & 50 & 50 & 33 & 23 & 18 & 17 & 18 & 12 & 6 & 19 & 28 & 37 \\
\hline 50 & 50 & 50 & 41 & 29 & 20 & 24 & 22 & 13 & 9 & 25 & 35 & 42 \\
\hline 60 & 50 & 50 & 50 & 32 & 28 & 31 & 28 & 16 & 15 & 33 & 42 & 50 \\
\hline 70 & 50 & 50 & 50 & 40 & 34 & 36 & 30 & 19 & 28 & 38 & 47 & 50 \\
\hline 80 & 50 & 50 & 50 & 50 & 48 & 50 & 33 & 28 & 38 & 50 & 50 & 50 \\
\hline 90 & 50 & 50 & 50 & 50 & 50 & 50 & 38 & 34 & 50 & 50 & 50 & 50 \\
\hline 99,9 & 50 & 50 & 50 & 50 & 50 & 50 & so & 50 & 50 & 50 & 50 & 50 \\
\hline MEDIA & 44 & 44 & 39 & 31 & 27 & 28 & 24 & 19 & 21 & 28 & 34 & 40 \\
\hline MEDIANA & 50 & 50 & 46 & 30 & 24 & 28 & 25 & 15 & 12 & 29 & 39 & 46 \\
\hline
\end{tabular}


Continuação:

Tabela 22- Probabilidades (\%) do armazenamento hídrico decendial, com 10, 20, 30, 40, $50,60,70,80,90$ e $99 \%$ de ocorrências para a CAD: $50 \mathrm{~mm}$, com valores menores ou iguais, referentes aos dados abaixo.

\begin{tabular}{|c|c|c|c|c|c|c|c|c|c|c|c|c|}
\hline \multirow{2}{*}{$\frac{\text { PROB (\%) }}{10}$} & \multicolumn{12}{|c|}{$2^{\circ}$ Decéndlo } \\
\hline & 20 & 17 & 10 & 5 & 4 & 2 & 4 & 2 & 2 & 5 & 7 & 19 \\
\hline 20 & 32 & 28 & 18 & 11 & 7 & 5 & 7 & 6 & 3 & 12 & 14 & 34 \\
\hline 30 & 42 & 31 & 28 & 16 & 13 & 12 & 14 & 8 & 6 & 24 & 22 & 41 \\
\hline 40 & 50 & 41 & 33 & 19 & 20 & 17 & 19 & 9 & 10 & 29 & 26 & 49 \\
\hline 50 & 50 & 50 & 43 & 25 & 23 & 23 & 22 & 10 & 13 & 35 & 34 & 50 \\
\hline 60 & 50 & 50 & 50 & 29 & 30 & 37 & 24 & 14 & 20 & 39 & 47 & 50 \\
\hline 70 & 50 & 50 & 50 & 34 & 35 & 40 & 27 & 16 & 29 & 45 & 50 & 50 \\
\hline 80 & 50 & 50 & 50 & 49 & 45 & 49 & 30 & 22 & 48 & 50 & 50 & 50 \\
\hline 90 & 50 & 50 & 50 & 50 & 50 & 50 & 41 & 34 & 50 & 50 & 50 & 50 \\
\hline 99,9 & 50 & 50 & 50 & 50 & 50 & 50 & 50 & 50 & 50 & 50 & 50 & 50 \\
\hline MEDIA & 44 & 42 & 38 & 29 & 28 & 29 & 24 & 17 & 23 & 34 & 35 & 44 \\
\hline MEDIANA & 50 & 50 & 46 & 27 & 26 & 30 & 23 & 12 & 17 & 37 & 40 & 50 \\
\hline PROB (\%) & \multicolumn{11}{|c|}{$3^{\circ}$ Decêndio } & \\
\hline 10 & 19 & 16 & 8 & 4 & 3 & 2 & 3 & 1 & 1 & 4 & 10 & 23 \\
\hline 20 & 31 & 27 & 14 & 7 & 6 & 9 & 8 & 5 & 3 & 14 & 20 & 32 \\
\hline 30 & 45 & 37 & 23 & 13 & 10 & 12 & 12 & 6 & 6 & 21 & 26 & 41 \\
\hline 40 & 49 & 41 & 27 & 16 & 14 & 18 & 15 & 7 & 11 & 25 & 32 & 49 \\
\hline 50 & 50 & 50 & 36 & 19 & 22 & 22 & 17 & 9 & 18 & 28 & 40 & 50 \\
\hline 60 & 50 & 50 & 44 & 27 & 26 & 32 & 19 & 13 & 25 & 38 & 48 & 50 \\
\hline 70 & 50 & 50 & 50 & 33 & 34 & 35 & 22 & 23 & 33 & 46 & 50 & 50 \\
\hline 80 & 50 & 50 & 50 & 41 & 43 & 38 & 29 & 29 & 40 & 50 & 50 & 50 \\
\hline 90 & 50 & 50 & 50 & 48 & 50 & 41 & 39 & 40 & 50 & 50 & 50 & 50 \\
\hline 99,9 & 50 & 50 & 50 & 50 & 50 & 50 & 50 & 50 & 50 & 50 & 50 & 50 \\
\hline MEDIA & 44 & 42 & 35 & 26 & 26 & 26 & 21 & 18 & 24 & 33 & 38 & 45 \\
\hline MEDIANA & 50 & 50 & 40 & 23 & 24 & 27 & 18 & 11 & 22 & 33 & 44 & 50 \\
\hline
\end{tabular}

Constatou-se que nos três decêndios da CAD: $100 \mathrm{~mm}$ do armazenamento hídrico, os valores menores que $79 \mathrm{~mm}$ ocorreram com $20 \%$ de probabilidade e menores que $100 \mathrm{~mm}$, até $85 \mathrm{~mm}$, com $80 \%$ na estação chuvosa. Na estação seca, valores abaixo de $51 \mathrm{~mm}$ tiveram $20 \%$ de probabilidade de ocorrência, enquanto valores de $98 \mathrm{~mm}$ perfizeram $80 \%$, conforme Tabela 23 . 
Tabela 23- Probabilidades (\%) do armazenamento hídrico decendial, com 10, 20, 30, 40, $50,60,70,80,90$ e $99 \%$ de ocorrências para a CAD: $100 \mathrm{~mm}$, com valores menores ou iguais, referentes aos dados abaixo.

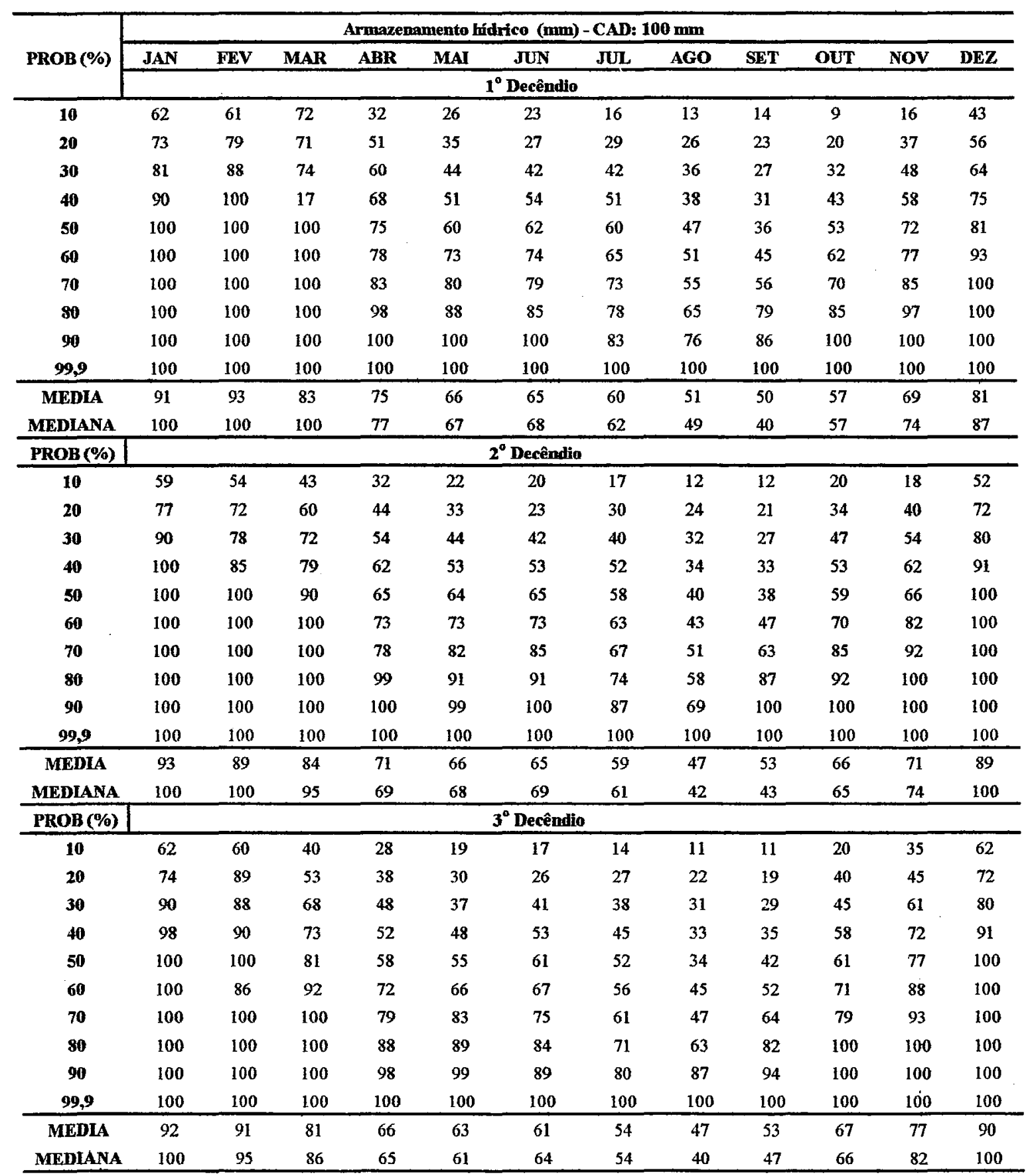


Para a CAD de $150 \mathrm{~mm}$ observou-se que, nos três decêndios (Tabela 24), os valores menores que $125 \mathrm{~mm}$ ocorreram com $20 \%$ de probabilidade e valores abaixo de $150 \mathrm{~mm}$ com $80 \%$ de probabilidade na estação chuvosa e na estação seca, com $20 \%$ de probabilidades, os valores foram abaixo de $90 \mathrm{~mm}$ e menores que $149 \mathrm{~mm}$ com $80 \%$ de probabilidade de ocorrência.

Tabela 24- Probabilidades (\%) do armazenamento hídrico decendial, com 10, 20, 30, 40, $50,60,70,80,90$ e $99 \%$ de ocorrências para a CAD: $150 \mathrm{~mm}$, com valores menores ou iguais, referentes aos dados abaixo.

\begin{tabular}{|c|c|c|c|c|c|c|c|c|c|c|c|}
\hline \multirow{3}{*}{ PROB (\%) } & \multicolumn{10}{|c|}{ Armazenamento hidrico (mm) - CAD: $150 \mathrm{~mm}$} & \multirow[b]{2}{*}{ NOV } \\
\hline & JAN & FEV & MAR & ABR & MAI & JUN & JUL & AGO & SET & OUT & \\
\hline & \multicolumn{11}{|c|}{$1^{\circ}$ Decêndio } \\
\hline 10 & 98 & 106 & 92 & 71 & 61 & 50 & 39 & 33 & 38 & 27 & 40 \\
\hline 20 & 118 & 125 & 111 & 90 & 72 & 60 & 64 & 58 & 54 & 45 & 61 \\
\hline 30 & 126 & 137 & 119 & 105 & 85 & 74 & 74 & 69 & 60 & 58 & 73 \\
\hline 40 & 140 & 150 & 130 & 116 & 92 & 97 & 87 & 76 & 64 & 75 & 89 \\
\hline 50 & 150 & 150 & 138 & 124 & 107 & 107 & 102 & 84 & 66 & 85 & 107 \\
\hline 60 & 150 & 150 & 150 & 127 & 118 & 117 & 108 & 91 & 80 & 96 & 120 \\
\hline 70 & 150 & 150 & 150 & 131 & 129 & 125 & 118 & 97 & 95 & 107 & 132 \\
\hline 80 & 150 & 150 & 150 & 148 & 135 & 134 & 125 & 105 & 114 & 124 & 144 \\
\hline 90 & 150 & 150 & 150 & 150 & 150 & 150 & 133 & 122 & 130 & 146 & 150 \\
\hline 99,9 & 150 & 150 & 150 & 150 & 150 & 150 & 150 & 150 & 150 & 150 & 150 \\
\hline MEDIA & 138 & 142 & 134 & 121 & 110 & 106 & 100 & 88 & 85 & 91 & 107 \\
\hline MEDLANA & 150 & 150 & 144 & 125 & 112 & 112 & 105 & 88 & 73 & 91 & 114 \\
\hline PROR (\%) & \multicolumn{11}{|c|}{$2^{\circ}$ Decêndio } \\
\hline 10 & 101 & 100 & 85 & 71 & 53 & 46 & 41 & 31 & 33 & 48 & 33 \\
\hline 20 & 123 & 118 & 106 & 86 & 70 & 55 & 61 & 52 & 49 & 58 & 72 \\
\hline 30 & 137 & 127 & 118 & 98 & 86 & 83 & 78 & 64 & 53 & 75 & 87 \\
\hline 40 & 150 & 134 & 128 & 109 & 96 & 96 & 90 & 72 & 62 & 85 & 98 \\
\hline 50 & 150 & 150 & 141 & 112 & 109 & 108 & 98 & 76 & 74 & 91 & 111 \\
\hline 60 & 150 & 150 & 150 & 122 & 120 & 113 & 109 & 84 & 86 & 100 & 118 \\
\hline 70 & 150 & 150 & 150 & 127 & 131 & 129 & 114 & 88 & 102 & 119 & 135 \\
\hline 80 & 150 & 150 & 150 & 149 & 140 & 139 & 121 & 98 & 117 & 138 & 150 \\
\hline 90 & 150 & 150 & 150 & 150 & 145 & 150 & 131 & 117 & 145 & 150 & 150 \\
\hline 99,9 & 150 & 150 & 150 & 150 & 150 & 150 & 150 & 150 & 150 & 150 & 150 \\
\hline MEDLA & 141 & 138 & 133 & 117 & 110 & 107 & 99 & 83 & 87 & 101 & 110 \\
\hline MEDIANA & 150 & 150 & 145 & 117 & 114 & 111 & 103 & 80 & 80 & 96 & 114 \\
\hline
\end{tabular}


Continuação:

Tabela 24- Probabilidades (\%) do armazenamento hídrico decendial, com 10, 20, 30, 40, $50,60,70,80,90$ e $99 \%$ de ocorrências para a CAD: $150 \mathrm{~mm}$, com valores menores ou iguais, referentes aos dados abaixo.

\begin{tabular}{ccccccccccccc} 
PROB (\%) & \multicolumn{10}{c}{$\mathbf{3}^{\circ}$ Decêndio } \\
\hline $\mathbf{1 0}$ & 104 & 91 & $\mathbf{8 1}$ & 59 & 49 & 42 & 37 & 32 & 31 & 45 & 58 & 96 \\
20 & 119 & 118 & 98 & 79 & 67 & 59 & 59 & 50 & 45 & 64 & 77 & 115 \\
30 & 136 & 133 & 116 & 88 & 74 & 79 & 74 & 65 & 56 & 75 & 95 & 122 \\
40 & 146 & 138 & 122 & 97 & 82 & 95 & 84 & 68 & 65 & 85 & 111 & 137 \\
50 & 150 & 149 & 131 & 104 & 101 & 102 & 91 & 71 & 75 & 92 & 120 & 150 \\
60 & 150 & 150 & 141 & 114 & 114 & 110 & 98 & 74 & 88 & 115 & 130 & 150 \\
70 & 150 & 150 & 150 & 128 & 132 & 121 & 106 & 87 & 98 & 122 & 139 & 150 \\
80 & 150 & 150 & 150 & 137 & 137 & 133 & 111 & 106 & 116 & 140 & 150 & 150 \\
90 & 150 & 150 & 150 & 148 & 149 & 138 & 125 & 131 & 144 & 150 & 150 & 150 \\
99,9 & 150 & 150 & 150 & 150 & 150 & 150 & 150 & 150 & 150 & 150 & 150 & 150 \\
\hline MEDIA & 140 & 138 & 129 & 110 & 105 & 103 & 94 & 83 & 87 & 104 & 118 & 137 \\
MEDIANA & 150 & 149 & 136 & 109 & 107 & 106 & 95 & 73 & 82 & 103 & 125 & 150 \\
\hline
\end{tabular}

A Tabela 25 apresenta os valores da deficiência hídrica mensal, na qual verificouse $20 \%$ de probabilidade de ocorrência para valor igual a $0 \mathrm{~mm}$ nos três níveis de $\mathrm{CAD}$, tanto na estação chuvosa quanto na estação seca. Para $80 \%$ de probabilidade, verificaram-se valores menores que 27, 23 e $19 \mathrm{~mm}$ para as CAD de 50,100 e $150 \mathrm{~mm}$, respectivamente, na estação chuvosa. Na estação seca, os valores foram menores que 54, 47 e $38 \mathrm{~mm}$ nas CAD de 50,100 e $150 \mathrm{~mm}$, respectivamente. 
Tabela 25- Probabilidades (\%) da deficiência hídrica mensal, com 10, 20, 30, 40, 50, 60, $70,80,90$ e $99 \%$ de ocorrências para a CAD: 50,100 e $150 \mathrm{~mm}$, com valores menores ou iguais, referentes aos dados abaixo.

\begin{tabular}{|c|c|c|c|c|c|c|c|c|c|c|c|c|}
\hline \multirow[b]{2}{*}{ PROB (\%) } & \multicolumn{10}{|c|}{ Deficiência hidrica mensal (mm) - CAD: $50 \mathrm{~mm}$} & \multirow[b]{2}{*}{ Nov } & \multirow[b]{2}{*}{ DEZ } \\
\hline & JAN & FEV & MAR & ABBR & MAI & JUN & JUL & AGO & SET & OUT & & \\
\hline 10 & 0 & $\mathbf{0}$ & 0 & $\mathbf{0}$ & 0 & 0 & 0 & 0 & $\mathbf{0}$ & $\mathbf{0}$ & 0 & $\mathbf{0}$ \\
\hline 20 & 0 & o & 0 & 0 & 0 & 0 & 0 & 0 & 0 & 0 & 0 & 0 \\
\hline 30 & 0 & 0 & $\mathbf{0}$ & $\mathbf{0}$ & 0 & 0 & 7 & 11 & $\mathbf{0}$ & 0 & 0 & 0 \\
\hline 40 & $\mathbf{0}$ & $\mathbf{0}$ & 0 & 1 & 2 & 1 & 11 & 20 & 5 & 0 & $\mathbf{0}$ & 0 \\
\hline 50 & 0 & 0 & 0 & 8 & 7 & 6 & 16 & 31 & 16 & 0 & 0 & 0 \\
\hline 60 & $\mathbf{0}$ & 0 & 0 & 17 & 14 & 13 & 19 & 36 & 28 & 2 & $\mathbf{0}$ & 0 \\
\hline 70 & 0 & 0 & 3 & 25 & 21 & 17 & 23 & 42 & 41 & 12 & 4 & 0 \\
\hline 80 & 0 & 2 & 18 & 32 & 30 & 24 & 30 & 46 & 54 & 27 & 19 & 0 \\
\hline 90 & 3 & 26 & 39 & 41 & 46 & 30 & 42 & 54 & 67 & 37 & 33 & 3 \\
\hline 99,9 & 126 & 112 & 79 & 80 & 83 & 60 & 58 & 67 & 102 & 97 & 83 & 105 \\
\hline MEDIA & 13 & 14 & 14 & 20 & 20 & 15 & 21 & 31 & 31 & 18 & 14 & 11 \\
\hline \multirow[t]{2}{*}{ MEDIANA } & 0 & 0 & 0 & 13 & 11 & 9 & 18 & 33 & 22 & 1 & 0 & 0 \\
\hline & \multicolumn{12}{|c|}{ CAD: $100 \mathrm{~mm}$} \\
\hline PROB (\%) & JAN & FEV & MAR & ABR & MAI & JuN & JUL & ACO & SET & out & NOV & $\mathrm{DEZ}$ \\
\hline 10 & 0 & 0 & 0 & 0 & 0 & 0 & 0 & 0 & 0 & 0 & 0 & 0 \\
\hline 20 & 0 & 0 & 0 & 0 & 0 & 0 & 0 & 0 & 0 & 0 & 0 & 0 \\
\hline 30 & 0 & 0 & 0 & 0 & 0 & 0 & 4 & 7 & 0 & 0 & 0 & 0 \\
\hline 40 & 0 & 0 & 0 & 1 & 1 & 0 & 7 & 15 & 4 & 0 & 0 & 0 \\
\hline 50 & 0 & 0 & 0 & 5 & 4 & 3 & 11 & 23 & 14 & 0 & 0 & 0 \\
\hline 60 & 0 & 0 & 0 & 10 & 9 & 8 & 14 & 28 & 24 & 2 & 0 & 0 \\
\hline 70 & 0 & 0 & 2 & 15 & 15 & 13 & 18 & 31 & 33 & 8 & 2 & 0 \\
\hline 80 & 0 & 1 & 11 & 19 & 21 & 16 & 22 & 35 & 47 & 23 & 12 & 0 \\
\hline so & 2 & 16 & 27 & 32 & 34 & 23 & 32 & 45 & 58 & 31 & 24 & 4 \\
\hline 99,9 & 114 & 109 & 71 & 61 & 60 & 57 & 53 & 64 & 100 & 81 & 75 & 80 \\
\hline MEDIA & 12 & 13 & 11 & 14 & 14 & 12 & 16 & 25 & 28 & 14 & 11 & 8 \\
\hline \multirow[t]{2}{*}{ MEDIANA } & 0 & 0 & 0 & 7 & 7 & 6 & 12 & 25 & 19 & 1 & 0 & 0 \\
\hline & \multicolumn{12}{|c|}{ CAD: $150 \mathrm{~mm}$} \\
\hline $\operatorname{PROB}(\%)$ & JAN & FEV & MAR & ABR & MAI & JUN & JUL & $A C O$ & SET & OUT & Nov & DEZ \\
\hline 10 & 0 & 0 & 0 & 0 & 0 & 0 & 0 & 0 & 0 & 0 & 0 & 0 \\
\hline 20 & 0 & 0 & 0 & 0 & $\mathbf{0}$ & 0 & 0 & 0 & 0 & 0 & 0 & 0 \\
\hline 30 & 0 & 0 & 0 & 0 & 0 & 0 & 3 & 6 & 0 & 0 & 0 & 0 \\
\hline 40 & 0 & 0 & 0 & 0 & 1 & 0 & 5 & 13 & 3 & 0 & 0 & 0 \\
\hline 50 & 0 & 0 & 0 & 4 & 3 & 2 & 7 & 18 & 13 & 0 & 0 & 0 \\
\hline 60 & 0 & 0 & 0 & 7 & 6 & 6 & 10 & 22 & 19 & 1 & 0 & 0 \\
\hline 70 & 0 & 0 & 2 & 10 & 12 & 9 & 15 & 25 & 26 & 5 & 2 & 0 \\
\hline 80 & 0 & 1 & 7 & 14 & 18 & 13 & 17 & 29 & 38 & 19 & 9 & 0 \\
\hline 90 & 2 & 12 & 20 & 26 & 27 & 19 & 27 & 40 & 49 & 26 & 17 & 3 \\
\hline 99,9 & 100 & 102 & 61 & 49 & 46 & 53 & 49 & 59 & 95 & 67 & 65 & 63 \\
\hline MEDIA & 10 & 11 & 9 & 11 & 11 & 10 & 13 & 21 & 24 & 12 & 9 & 7 \\
\hline MEDIANA & 0 & 0 & 0 & 5 & 5 & 4 & 9 & 20 & 16 & 1 & 0 & 0 \\
\hline
\end{tabular}


Nas Tabelas 26, 27 e 28 apresentam-se os valores da deficiência hídrica nos três decêndios das CAD: 50,100 e $150 \mathrm{~mm}$. Observou-se que, com 20\% de probabilidade, os valores são iguais a $0 \mathrm{~mm}$ na estação chuvosa e menores de $3 \mathrm{~mm}$ na estação seca, enquanto que para $80 \%$ de probabilidade ocorreram valores abaixo de 14,16 e $38 \mathrm{~mm}$ na estação chuvosa e 21, 18 e $16 \mathrm{~mm}$ na estação seca, considerando-se as CAD: 50,100 e $150 \mathrm{~mm}$, respectivamente.

Tabela 26- Probabilidades (\%) da deficiência hídrica decendial, com 10, 20,30, 40,50, $60,70,80,90$ e $99 \%$ de ocorrências para a CAD: 50, com valores menores ou iguais, referentes aos dados abaixo.

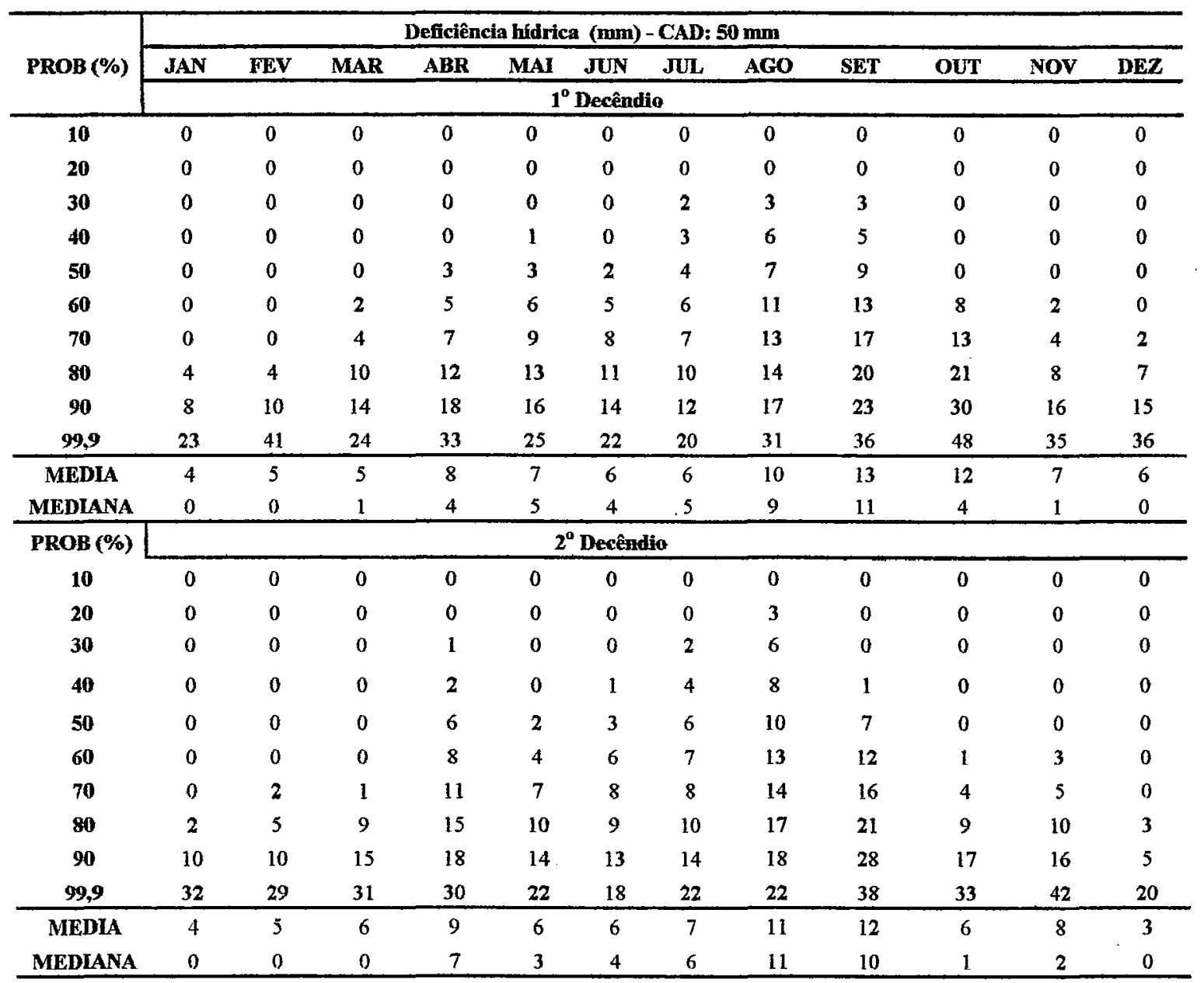


Continuação:

Tabela 26- Probabilidades (\%) da deficiência hídrica decendial, com 10,20,30,40,50, $60,70,80,90$ e $99 \%$ de ocorrências para a CAD: $50 \mathrm{~mm}$, com valores menores ou iguais, referentes aos dados abaixo.

\begin{tabular}{|c|c|c|c|c|c|c|c|c|c|c|c|c|}
\hline \multirow[b]{2}{*}{ PROB (\%) } & \multicolumn{11}{|c|}{$3^{0}$ Decêndio } & \multirow[b]{2}{*}{ DEZ } \\
\hline & JAN & FEV & MAR & ABR & MAI & JUN & JUL & AGO & SET & OUT & NOV & \\
\hline 10 & 0 & 0 & 0 & 0 & 0 & 0 & 0 & 0 & 0 & 0 & 0 & 0 \\
\hline 20 & 0 & 0 & 0 & 0 & 0 & 0 & 1 & 0 & 0 & 0 & 0 & 0 \\
\hline 30 & 0 & 0 & 0 & 1 & 1 & 1 & 5 & 2 & 0 & 0 & 0 & 0 \\
\hline 40 & 0 & 0 & 0 & 4 & 2 & 2 & 7 & 7 & 1 & 0 & 0 & 0 \\
\hline 50 & 0 & 0 & 1 & 6 & 5 & 3 & 9 & 9 & 3 & 0 & 0 & 0 \\
\hline 60 & 0 & 0 & 6 & 10 & 9 & 5 & 10 & 12 & 7 & 4 & 1 & 0 \\
\hline 70 & 0 & 1 & 8 & 12 & 12 & 8 & 11 & 17 & 11 & 8 & 2 & 0 \\
\hline 80 & 1 & 3 & 14 & 16 & 13 & 10 & 13 & 20 & 20 & 11 & 6 & 4 \\
\hline 90 & 6 & 12 & 21 & 21 & 19 & 13 & 15 & 25 & 26 & 21 & 18 & 8 \\
\hline 99,9 & 22 & 35 & 37 & 38 & 27 & 19 & 24 & 31 & 40 & 36 & 34 & 28 \\
\hline MEDIA & 3 & 5 & 9 & 11 & 9 & 6 & 9 & 12 & 11 & 8 & 6 & 4 \\
\hline MEDIANA & 0 & 0 & 3 & 8 & 7 & 4 & 9 & 11 & 5 & 2 & 0 & 0 \\
\hline
\end{tabular}

Tabela 27-Probabilidades (\%) da deficiência hídrica decendial, com 10, 20,30,40, 50, $60,70,80,90$ e $99 \%$ de ocorrências para a CAD: $100 \mathrm{~mm}$, com valores menores ou iguais, referentes aos dados abaixo.

\begin{tabular}{|c|c|c|c|c|c|c|c|c|c|c|c|c|}
\hline \multirow{3}{*}{ PROB (\%) } & \multicolumn{12}{|c|}{ Deficiêneia hidrica (mm) - CAD: $100 \mathrm{~mm}$} \\
\hline & JAN & FEV & MAR & ABR & MAI & JUN & JUL & AGO & SET & OUT & Nov & DEZ \\
\hline & \multicolumn{12}{|c|}{$1^{\circ}$ Decêndio } \\
\hline 10 & 0 & 0 & $\mathbf{0}$ & 0 & 0 & 0 & 0 & 0 & 0 & 0 & $\mathbf{0}$ & 0 \\
\hline 20 & 0 & 0 & 0 & 0 & 0 & 0 & 0 & 0 & 0 & 0 & 0 & 0 \\
\hline 30 & 0 & 0 & 0 & $\mathbf{0}$ & 0 & 0 & 1 & 2 & 2 & 0 & 0 & 0 \\
\hline 40 & 0 & 0 & 0 & 0 & 1 & 0 & 2 & 5 & 5 & 0 & 0 & 0 \\
\hline 50 & 0 & 0 & 0 & 3 & 2 & 2 & 2 & 6 & 7 & 0 & 0 & 0 \\
\hline 60 & 0 & 0 & 1 & 3 & 4 & 4 & 4 & 8 & 10 & 5 & 1 & 0 \\
\hline 70 & 0 & 0 & 2 & 5 & 7 & 5 & 6 & 9 & 15 & 10 & 2 & 2 \\
\hline 80 & 3 & 2 & 6 & 7 & 9 & 9 & 8 & 11 & 16 & 16 & 7 & 5 \\
\hline 90 & 5 & 6 & 8 & 11 & 12 & 11 & 10 & 15 & 19 & 23 & 15 & 10 \\
\hline 99,9 & 14 & 32 & 16 & 27 & 20 & 19 & 19 & 28 & 35 & 47 & 34 & 28 \\
\hline MEDIA & 2 & 4 & 3 & 6 & 5 & 5 & 5 & 8 & 11 & 10 & 6 & 4 \\
\hline MEDIANA & 0 & 0 & 1 & 3 & $\mathbf{3}$ & 3 & 3 & 7 & 9 & 3 & 1 & 0 \\
\hline
\end{tabular}


Continuação:

Tabela 27- Probabilidades (\%) da deficiência hídrica decendial, com 10, 20, 30, 40, 50, $60,70,80,90$ e $99 \%$ de ocorrências para a CAD: $100 \mathrm{~mm}$, com valores menores ou iguais, referentes aos dados abaixo.

\begin{tabular}{|c|c|c|c|c|c|c|c|c|c|c|c|c|}
\hline \multicolumn{13}{|c|}{$2^{\circ}$ Decêndio } \\
\hline PROB (\%) & JAN & FEV & MAR & ABR & MAI & JUN & JUL & AGO & SET & OUT & Nov & DEZ \\
\hline 10 & 0 & 0 & 0 & 0 & 0 & 0 & 0 & 0 & 0 & 0 & 0 & 0 \\
\hline 20 & 0 & 0 & 0 & 0 & 0 & 0 & 0 & 3 & 0 & 0 & 0 & 0 \\
\hline 30 & 0 & 0 & 0 & 0 & 0 & 0 & 1 & 5 & 0 & 0 & 0 & 0 \\
\hline 40 & 0 & 0 & 0 & 2 & 0 & 1 & 3 & 7 & 1 & 0 & 0 & 0 \\
\hline 50 & 0 & 0 & 0 & 3 & 1 & 2 & 4 & 8 & 8 & 0 & 0 & 0 \\
\hline 60 & 0 & 0 & 0 & 6 & 3 & 5 & 5 & 9 & 10 & 1 & 3 & 0 \\
\hline 70 & 0 & 1 & 0 & 7 & 5 & 6 & 6 & 11 & 14 & 4 & 5 & 0 \\
\hline 80 & 2 & 3 & 6 & 10 & 8 & 8 & 9 & 12 & 18 & 9 & 8 & 2 \\
\hline 90 & 6 & 6 & 11 & 14 & 12 & 12 & 10 & 16 & 23 & 13 & 11 & 4 \\
\hline 99,9 & 20 & 23 & 24 & 27 & 18 & 18 & 21 & 21 & 34 & 26 & 37 & 18 \\
\hline MEDIA & 3 & 3 & 4 & 7 & 5 & 5 & 6 & 9 & 11 & 5 & 6 & 2 \\
\hline MEDIANA & 0 & 0 & 0 & 5 & 2 & 3 & 4 & 9 & 9 & 0 & 1 & 0 \\
\hline PROB (\%) & \multicolumn{12}{|c|}{$3^{\circ}$ Decêndio } \\
\hline 10 & 0 & 0 & 0 & 0 & 0 & 0 & 0 & 0 & 0 & 0 & 0 & 0 \\
\hline 20 & 0 & 0 & 0 & 0 & 0 & 0 & 1 & 0 & 0 & a & $\mathbf{0}$ & 0 \\
\hline 30 & 0 & 0 & 0 & 0 & 1 & 1 & 3 & 1 & 0 & 0 & 0 & 0 \\
\hline 40 & 0 & 0 & 0 & 2 & 1 & 1 & 6 & 5 & 1 & 0 & 0 & 0 \\
\hline 50 & 0 & 0 & 1 & 4 & 4 & 2 & 6 & 8 & 2 & 1 & 0 & 0 \\
\hline 60 & 0 & 0 & 4 & 6 & 7 & 4 & 7 & 11 & 6 & 4 & 0 & 0 \\
\hline 70 & 0 & 0 & 5 & 8 & 9 & 6 & 9 & 13 & 10 & 5 & 2 & 0 \\
\hline 80 & 1 & 2 & 9 & 11 & 11 & 8 & 11 & 17 & 14 & 11 & 5 & 2 \\
\hline 90 & 3 & 7 & 14 & 15 & 14 & 11 & 12 & 19 & 23 & 21 & 14 & 5 \\
\hline 99,9 & 13 & 29 & 29 & 31 & 25 & 17 & 23 & 30 & 34 & 30 & 33 & 21 \\
\hline MEDIA & 2 & 4 & 6 & 8 & 7 & 5 & 8 & 10 & 9 & 7 & 5 & 3 \\
\hline MEDIANA & 0 & 0 & 2 & 5 & 5 & 3 & 7 & 10 & 4 & 2 & 0 & 0 \\
\hline
\end{tabular}


Tabela 28- Probabilidades (\%) da deficiência hídrica decendial, com 10, 20, 30, 40, 50, $60,70,80,90$ e $99 \%$ de ocorrências para a CAD: $150 \mathrm{~mm}$, com valores menores ou iguais, referentes aos dados abaixo.

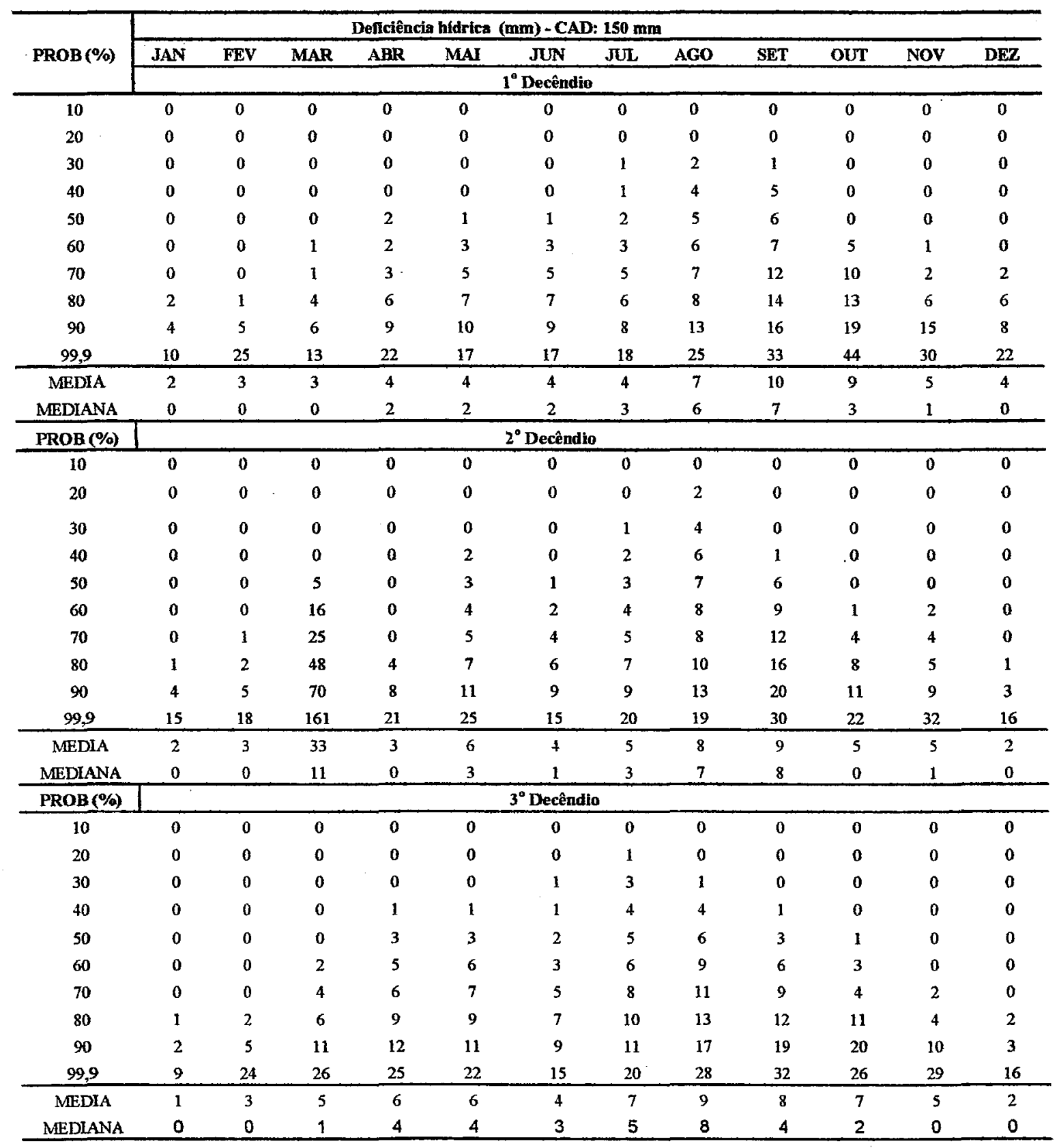


O excedente hídrico mensal (Tabela 29) apresentou $20 \%$ de probabilidade de ocorrência para valor igual a $0 \mathrm{~mm}$ nos três níveis de $\mathrm{CAD}$, tanto na estação chuvosa quanto na estação seca. Para $80 \%$ de probabilidade, verificaram-se valores menores que $177,172,172 \mathrm{~mm}$ para as $\mathrm{CAD}$ de 50,100 e $150 \mathrm{~mm}$, respectivamente, na estação chuvosa. Na estação seca, os valores foram menores que $16 \mathrm{~mm}$ para a CAD de $50 \mathrm{~mm}$, $16 \mathrm{~mm}$ na CAD de $100 \mathrm{~mm}$ e $15 \mathrm{~mm}$ na CAD de $150 \mathrm{~mm}$, respectivamente. Ressalta-se que o mês de outubro apresentou $0 \mathrm{~mm}$ para a CAD de $150 \mathrm{~mm}$.

Tabela 29- Probabilidades (\%) do excedente hídrico mensal, com 10, 20, 30, 40, 50, 60, $70,80,90$ e $99 \%$ de ocorrências para a $\mathrm{CAD}: 50,100$ e $150 \mathrm{~mm}$, com valores menores ou iguais, referentes aos dados abaixo.

\begin{tabular}{|c|c|c|c|c|c|c|c|c|c|c|c|c|}
\hline \multirow[b]{2}{*}{ PROB (\%) } & \multicolumn{10}{|c|}{ Excedente hidrico menssl (mm) - CAD: $50 \mathrm{~mm}$} & \multirow[b]{2}{*}{ Nov } & \multirow[b]{2}{*}{ DEZ } \\
\hline & JAN & FEV & MAR & ABR & MAI & JUN & JUL & AGO & SET & OUT & & \\
\hline 10 & 0 & 0 & $\mathbf{0}$ & $\mathbf{0}$ & $\mathbf{0}$ & 0 & 0 & 0 & 0 & $\mathbf{0}$ & 0 & $\mathbf{0}$ \\
\hline 20 & 0 & 0 & 0 & $\mathbf{0}$ & 0 & 0 & 0 & $\mathbf{0}$ & 0 & 0 & 0 & 0 \\
\hline 30 & 24 & 0 & 0 & 0 & 0 & 0 & $\mathbf{0}$ & 0 & $\mathbf{0}$ & $\mathbf{0}$ & 0 & 19 \\
\hline 40 & 51 & 30 & 5 & 0 & $\mathbf{0}$ & 0 & 0 & 0 & $\mathbf{0}$ & $\mathbf{0}$ & $\mathbf{0}$ & 32 \\
\hline 50 & 85 & 61 & 23 & o & $\mathbf{0}$ & 0 & 0 & 0 & $\mathbf{0}$ & $\mathbf{0}$ & $\mathbf{0}$ & 50 \\
\hline 60 & 128 & 85 & 41 & 0 & 0 & $\mathbf{0}$ & 0 & 0 & 0 & $\mathbf{0}$ & 12 & 76 \\
\hline 70 & 147 & 110 & 58 & 0 & 0 & $\mathbf{0}$ & 0 & 0 & 0 & 8 & 26 & 93 \\
\hline 80 & 177 & 151 & 76 & 16 & 5 & 0 & 0 & 0 & 0 & 35 & 47 & 115 \\
\hline 90 & 197 & 219 & 106 & 40 & 28 & 36 & 5 & 0 & 37 & 64 & 99 & 156 \\
\hline 99,9 & 369 & 362 & 328 & 154 & 260 & 125 & 70 & 31 & 150 & 133 & 161 & 261 \\
\hline MEDIA & 118 & 102 & 64 & 21 & 29 & 16 & 8 & 3 & 19 & 24 & 34 & 80 \\
\hline \multirow[t]{2}{*}{ MEDIANA } & 107 & 73 & 32 & 0 & 0 & 0 & 0 & 0 & 0 & 0 & 6 & 63 \\
\hline & \multicolumn{12}{|c|}{ CAD: $100 \mathrm{~mm}$} \\
\hline PROB (\%) & JAN & FEV & MAR & $\mathbf{A B R}$ & MAI & JUN & JUL & AGO & SET & OUT & Nov & DEZ \\
\hline 10 & 0 & 0 & 0 & 0 & 0 & 0 & 0 & 0 & 0 & 0 & 0 & 0 \\
\hline 20 & 0 & 0 & $\mathbf{0}$ & 0 & 0 & 0 & 0 & o & 0 & 0 & 0 & $\mathbf{0}$ \\
\hline 30 & 20 & 0 & 0 & 0 & 0 & 0 & 0 & 0 & $\mathbf{0}$ & 0 & 0 & 5 \\
\hline 40 & 47 & 30 & 0 & $\mathbf{0}$ & 0 & 0 & 0 & 0 & 0 & 0 & 0 & 23 \\
\hline 50 & 81 & 52 & 21 & $\mathbf{0}$ & $\mathbf{0}$ & 0 & 0 & 0 & 0 & D & $\mathbf{0}$ & 45 \\
\hline 60 & 128 & 85 & 38 & 0 & 0 & 0 & 0 & 0 & 0 & 0 & 0 & 64 \\
\hline 70 & 147 & 110 & 56 & 0 & 0 & 0 & 0 & 0 & 0 & $\mathbf{0}$ & 12 & 81 \\
\hline 80 & 172 & 151 & 75 & 16 & 3 & 0 & 0 & 0 & 0 & 11 & 37 & 101 \\
\hline 90 & 197 & 219 & 92 & 40 & 23 & 34 & 0 & 0 & 19 & 49 & 79 & 135 \\
\hline 99,9 & 369 & 362 & 328 & 154 & 260 & 125 & 62 & 31 & 117 & 120 & 127 & 250 \\
\hline MEDIA & 116 & 101 & 61 & 21 & 29 & 16 & 6 & 3 & 14 & 18 & 25 & 71 \\
\hline MEDLANA & 104 & 69 & 29 & 0 & 0 & 0 & 0 & 0 & $\mathbf{0}$ & 0 & 0 & 55 \\
\hline
\end{tabular}


Continuação:

Tabela 29- Probabilidades (\%) do excedente hídrico mensal, com 10, 20,30,40, 50, 60, $70,80,90$ e $99 \%$ de ocorrências para a CAD: 50,100 e $150 \mathrm{~mm}$, com valores menores ou iguais, referentes aos dados abaixo.

\begin{tabular}{|c|c|c|c|c|c|c|c|c|c|c|c|c|}
\hline \multirow[b]{2}{*}{ PROB (\%) } & \multicolumn{12}{|c|}{ CAD: $150 \mathrm{~mm}$} \\
\hline & JAN & HEV & MAR & ABR & MAI & JuN & JUL & AGO & SET & OUT & NOV & DEZ \\
\hline 10 & 0 & o & 0 & 0 & 0 & 0 & 0 & 0 & 0 & 0 & 0 & 0 \\
\hline 20 & 0 & 0 & 0 & 0 & 0 & 0 & 0 & 0 & 0 & 0 & 0 & 0 \\
\hline 30 & 16 & 0 & 0 & 0 & $\mathbf{0}$ & 0 & 0 & 0 & 0 & 0 & $\mathbf{0}$ & D \\
\hline 40 & 47 & 27 & 0 & 0 & 0 & 0 & 0 & 0 & 0 & 0 & 0 & 18 \\
\hline 50 & 79 & 50 & 21 & 0 & 0 & 0 & 0 & 0 & 0 & 0 & 0 & 32 \\
\hline 60 & 120 & 80 & 34 & 0 & 0 & $\mathbf{0}$ & 0 & 0 & 0 & 0 & $\mathbf{0}$ & 53 \\
\hline 70 & 147 & 108 & 56 & 0 & 0 & 0 & 0 & 0 & 0 & 0 & 0 & 66 \\
\hline 80 & 172 & 151 & 75 & 15 & 0 & 0 & 0 & 0 & 0 & 0 & 18 & 93 \\
\hline 90 & 191 & 219 & 91 & 40 & 21 & 30 & 0 & 0 & 13 & 33 & 64 & 117 \\
\hline 99,9 & 369 & 362 & 328 & 154 & 260 & 125 & 59 & 31 & 97 & 120 & 127 & 250 \\
\hline MEDIA & 114 & 100 & 60 & 21 & 28 & 15 & 6 & 3 & 11 & 15 & 21 & 63 \\
\hline MEDIANA & 100 & 65 & 28 & 0 & 0 & 0 & 0 & 0 & 0 & 0 & 0 & 42 \\
\hline
\end{tabular}

O excedente hídrico (Tabelas 30, 31 e 32), nos três decêndios, apresentou valores iguais a $0 \mathrm{~mm}$ para os três níveis de CAD com $20 \%$ de probabilidade; para $80 \%$ os valores foram iguais a $0 \mathrm{~mm}$ na estação seca. Somente no $1^{\circ}$ decêndio da CAD: $50 \mathrm{~mm}$ ocorreu valor menor que $6 \mathrm{~mm}$. Para a CAD: $100 \mathrm{~mm}$, na estação chuvosa, com $80 \%$ de probabilidade, os valores foram abaixo de 71,85 e $63 \mathrm{~mm}$, no $1^{\circ}, 2^{\circ}$ e $3^{\circ}$ decêndio respectivamente. $\mathrm{Na} C A D: 150 \mathrm{~mm}$ os valores foram abaixo de 68,85 e $58 \mathrm{~mm}$ no $1^{\circ}, 2^{\circ}$ e $3^{\circ}$ decêndio. 
Tabela 30- Probabilidades (\%) do excedente hídrico decendial, com 10, 20, 30, 40, 50, $60,70,80,90$ e $99 \%$ de ocorrências para a CAD: $50 \mathrm{~mm}$, com valores menores ou iguais, referentes aos dados abaixo.

\begin{tabular}{|c|c|c|c|c|c|c|c|c|c|c|c|c|}
\hline \multirow{3}{*}{ PROB (\%) } & \multicolumn{11}{|c|}{ Excedente hidrico (mm) - CAD: $50 \mathrm{~mm}$} & \multirow[b]{2}{*}{ DEZ } \\
\hline & JAN & FEV & MAR & $\mathbf{A B R}$ & MAI & JUN & JUL & AGO & SET & OUT & Nov & \\
\hline & \multicolumn{11}{|c|}{$1^{\circ}$ Decêndio } & \\
\hline 10 & 0 & 0 & 0 & 0 & 0 & 0 & 0 & 0 & 0 & 0 & 0 & 0 \\
\hline 20 & 0 & 0 & 0 & 0 & 0 & 0 & 0 & 0 & 0 & 0 & 0 & 0 \\
\hline 30 & 0 & 0 & 0 & 0 & 0 & 0 & 0 & 0 & 0 & 0 & 0 & 0 \\
\hline 40 & 4 & 4 & 0 & 0 & 0 & 0 & 0 & 0 & 0 & 0 & 0 & 0 \\
\hline $\mathbf{5 0}$ & 8 & 16 & 0 & 0 & 0 & 0 & 0 & 0 & 0 & 0 & 0 & 0 \\
\hline 60 & 24 & 29 & 14 & 0 & 0 & 0 & 0 & 0 & 0 & 0 & 0 . & 6 \\
\hline 70 & 38 & 42 & 25 & 0 & 0 & 0 & 0 & 0 & 0 & 0 & 0 & 20 \\
\hline 80 & 76 & 73 & 38 & 6 & 0 & 0 & 0 & 0 & 0 & 2 & 8 & 47 \\
\hline 90 & 102 & 136 & 61 & 17 & 7 & 5 & 0 & 0 & 7 & 27 & 15 & 72 \\
\hline 99,9 & 181 & 242 & 181 & 89 & 34 & 149 & 52 & 46 & 81 & 84 & 59 & 122 \\
\hline MEDIA & 43 & 54 & 32 & 11 & 4 & 15 & 5 & 5 & 9 & 11 & 8 & 27 \\
\hline MEDIANA & 16 & 23 & 7 & 0 & 0 & 0 & 0 & 0 & 0 & 0 & 0 & 3 \\
\hline PROB (\%) & \multicolumn{11}{|c|}{$\mathbf{2}^{\circ}$ Decêndio } & \\
\hline 10 & 0 & 0 & 0 & 0 & 0 & 0 & 0 & 0 & 0 & 0 & 0 & 0 \\
\hline 20 & 0 & 0 & 0 & 0 & 0 & 0 & 0 & 0 & 0 & 0 & 0 & 0 \\
\hline 30 & 0 & 0 & 0 & $\mathbf{0}$ & 0 & 0 & 0 & 0 & 0 & 0 & 0 & 0 \\
\hline 40 & 10 & 0 & 0 & 0 & 0 & 0 & 0 & 0 & 0 & 0 & 0 & 0 \\
\hline $\mathbf{5 0}$ & 24 & 9 & 0 & 0 & 0 & 0 & 0 & 0 & 0 & 0 & 0 & 7 \\
\hline 60 & 45 & 19 & 5 & 0 & 0 & 0 & 0 & 0 & 0 & 0 & 0 & 19 \\
\hline 70 & 55 & 35 & 13 & 0 & 0 & 0 & 0 & 0 & 0 & $\mathbf{0}$ & 1 & 31 \\
\hline 80 & 89 & 48 & 30 & 0 & 0 & 0 & 0 & 0 & 0 & 6 & 28 & 43 \\
\hline 90 & 114 & 70 & 50 & 25 & 7 & 15 & 0 & 0 & 28 & 38 & 50 & 76 \\
\hline 99,9 & 295 & 173 & 236 & 83 & 98 & 103 & 57 & 14 & 97 & 72 & 132 & 289 \\
\hline MEDIA & 63 & 35 & 33 & 11 & 11 & 12 & 6 & 1 & 13 & 12 & 21 & 47 \\
\hline MEDIANA & 35 & 14 & 3 & 0 & 0 & 0 & 0 & 0 & 0 & 0 & 0 & 13 \\
\hline PROB (\%) & \multicolumn{11}{|c|}{$3^{0}$ Decêndío } & \\
\hline 10 & 0 & 0 & 0 & 0 & 0 & 0 & 0 & 0 & 0 & 0 & 0 & 0 \\
\hline 20 & 0 & 0 & 0 & 0 & 0 & 0 & 0 & 0 & 0 & 0 & 0 & 0 \\
\hline 30 & 0 & 0 & 0 & 0 & 0 & 0 & 0 & 0 & 0 & 0 & 0 & 0 \\
\hline 40 & 0 & 0 & 0 & 0 & 0 & 0 & 0 & 0 & 0 & 0 & 0 & 0 \\
\hline $\mathbf{5 0}$ & 8 & 0 & 0 & 0 & 0 & 0 & 0 & 0 & 0 & 0 & 0 & 8 \\
\hline 60 & 27 & 18 & 0 & 0 & 0 & 0 & 0 & 0 & 0 & 0 & 0 & 22 \\
\hline 70 & 38 & 22 & 12 & 0 & 0 & 0 & 0 & 0 & 0 & 0 & 6 & 35 \\
\hline 80 & 59 & 46 & 35 & 0 & 0 & 0 & 0 & 0 & 0 & 15 & 31 & 76 \\
\hline 90 & 78 & 69 & 54 & 0 & 2 & 0 & 0 & 0 & 17 & 30 & 53 & 105 \\
\hline 99,9 & 197 & 167 & 145 & 78 & 239 & 133 & 41 & 48 & 112 & 97 & 119 & 145 \\
\hline MEDLA & 41 & 32 & 25 & 8 & 24 & 13 & 4 & 5 & 13 & 14 & 21 & 39 \\
\hline MEDIANA & 18 & 9 & 0 & 0 & 0 & 0 & 0 & 0 & 0 & 0 & 0 & 15 \\
\hline
\end{tabular}


Tabela 31- Probabilidades (\%) do excedente hídrico decendial, com 10, 20, 30, 40, 50, $60,70,80,90$ e $99 \%$ de ocorrências para a CAD: $100 \mathrm{~mm}$, com valores menores ou iguais, referentes aos dados abaixo.

\begin{tabular}{|c|c|c|c|c|c|c|c|c|c|c|c|c|}
\hline \multirow[b]{3}{*}{ PROB (\%) } & \multicolumn{11}{|c|}{ Excedente hídrico (mm) - CAD: $100 \mathrm{~mm}$} & \multirow[b]{3}{*}{ DEZ } \\
\hline & \multicolumn{11}{|c|}{$\begin{array}{l}1^{\circ} \text { Decêndilo } \\
\end{array}$} & \\
\hline & JAN & FEV & MAR & $\mathbf{A B R}$ & MAI & JUN & JUL & AGO & SET & OUT & NOV & \\
\hline 10 & 0 & 0 & 0 & 0 & 0 & 0 & 0 & 0 & 0 & 0 & 0 & 0 \\
\hline 20 & 0 & 0 & 0 & 0 & 0 & 0 & 0 & 0 & 0 & 0 & 0 & 0 \\
\hline 30 & 0 & 0 & 0 & 0 & 0 & 0 & 0 & 0 & 0 & 0 & 0 & 0 \\
\hline 40 & 0 & 2 & 0 & 0 & 0 & 0 & 0 & 0 & 0 & 0 & 0 & 0 \\
\hline 50 & 5 & 15 & 0 & 0 & 0 & 0 & 0 & 0 & 0 & 0 & 0 & 0 \\
\hline 60 & 22 & 27 & 5 & 0 & 0 & 0 & 0 & 0 & 0 & 0 & 0 & 0 \\
\hline 70 & 31 & 40 & 23 & 0 & 0 & 0 & 0 & 0 & 0 & 0 & 0 & 19 \\
\hline 80 & 66 & 71 & 37 & 0 & 0 & 0 & 0 & 0 & 0 & 0 & 0 & 29 \\
\hline 90 & 99 & 136 & 57 & 17 & 5 & 0 & 0 & 0 & 0 & 9 & 11 & 66 \\
\hline 99,9 & 181 & 240 & 180 & 88 & 27 & 149 & 49 & 46 & 57 & 61 & 59 & 122 \\
\hline MEDIA & 40 & 53 & 30 & 11 & 3 & 15 & 5 & 5 & 6 & 7 & 7 & 24 \\
\hline MEDIANA & 14 & 21 & 2 & 0 & 0 & 0 & 0 & 0 & 0 & 0 & 0 & 0 \\
\hline PROA (\%) & & & & & & Decêne & & & & & & \\
\hline 10 & 0 & 0 & 0 & 0 & 0 & 0 & 0 & 0 & 0 & 0 & 0 & 0 \\
\hline 20 & 0 & 0 & 0 & 0 & 0 & 0 & 0 & 0 & 0 & 0 & 0 & 0 \\
\hline 30 & 0 & 0 & 0 & 0 & 0 & 0 & 0 & 0 & 0 & 0 & 0 & 0 \\
\hline 40 & 7 & 0 & 0 & $\mathbf{0}$ & 0 & 0 & 0 & 0 & 0 & 0 & 0 & 0 \\
\hline 50 & 18 & 7 & 0 & 0 & 0 & 0 & 0 & 0 & 0 & 0 & 0 & 1 \\
\hline 60 & 43 & 16 & 5 & 0 & 0 & 0 & 0 & 0 & 0 & 0 & 0 & 14 \\
\hline 70 & 49 & 26 & 13 & 0 & 0 & 0 & 0 & 0 & 0 & 0 & 0 & 27 \\
\hline 80 & 85 & 48 & 27 & 0 & 0 & 0 & 0 & 0 & 0 & 0 & 9 & 37 \\
\hline 90 & 114 & 70 & 49 & 17 & 0 & 8 & 0 & 0 & 4 & 22 & 45 & 58 \\
\hline 99,9 & 294 & 164 & 236 & 80 & 81 & 78 & 47 & 2 & 97 & 64 & 132 & 277 \\
\hline MEDIA & 61 & 33 & 33 & 10 & 8 & 9 & 5 & 0 & 10 & 9 & 19 & 41 \\
\hline MEDIANA & 31 & 12 & 2 & 0 & 0 & 0 & 0 & 0 & 0 & 0 & 0 & 8 \\
\hline PROB (\%) & & & & & & Decên & & & & & & \\
\hline 10 & 0 & 0 & 0 & 0 & 0 & 0 & 0 & 0 & 0 & 0 & 0 & 0 \\
\hline 20 & 0 & 0 & 0 & 0 & 0 & 0 & 0 & 0 & 0 & 0 & 0 & 0 \\
\hline 30 & 0 & 0 & 0 & $\mathbf{0}$ & 0 & 0 & 0 & 0 & 0 & 0 & 0 & 0 \\
\hline 40 & 0 & 0 & 0 & 0 & 0 & 0 & 0 & 0 & 0 & 0 & 0 & 0 \\
\hline 50 & 8 & 0 & 0 & 0 & 0 & 0 & 0 & 0 & 0 & 0 & 0 & 7 \\
\hline 60 & 27 & 12 & 0 & 0 & 0 & 0 & 0 & 0 & 0 & 0 & 0 & 19 \\
\hline 70 & 38 & 21 & 11 & 0 & 0 & 0 & 0 & 0 & 0 & 0 & 0 & 35 \\
\hline 80 & 58 & 46 & 35 & 0 & 0 & 0 & 0 & 0 & 0 & 7 & 25 & 63 \\
\hline 90 & 78 & 69 & 44 & 0 & 0 & 0 & 0 & 0 & 0 & 19 & 52 & 102 \\
\hline 99,9 & 192 & 167 & 145 & 78 & 239 & 125 & 35 & 23 & 96 & 97 & 92 & 142 \\
\hline MEDIA & 40 & 31 & 24 & 8 & 24 & 13 & 4 & 2 & 10 & 12 & 17 & 37 \\
\hline MEDIANA & 17 & 6 & 0 & 0 & 0 & 0 & 0 & 0 & 0 & 0 & 0 & 13 \\
\hline
\end{tabular}


Tabela 32- Probabilidades (\%) do excedente hídrico decendial, com 10, 20, 30, 40, 50, $60,70,80,90$ e $99 \%$ de ocorrências para a CAD: $150 \mathrm{~mm}$, com valores menores ou iguais, referentes aos dados abaixo.

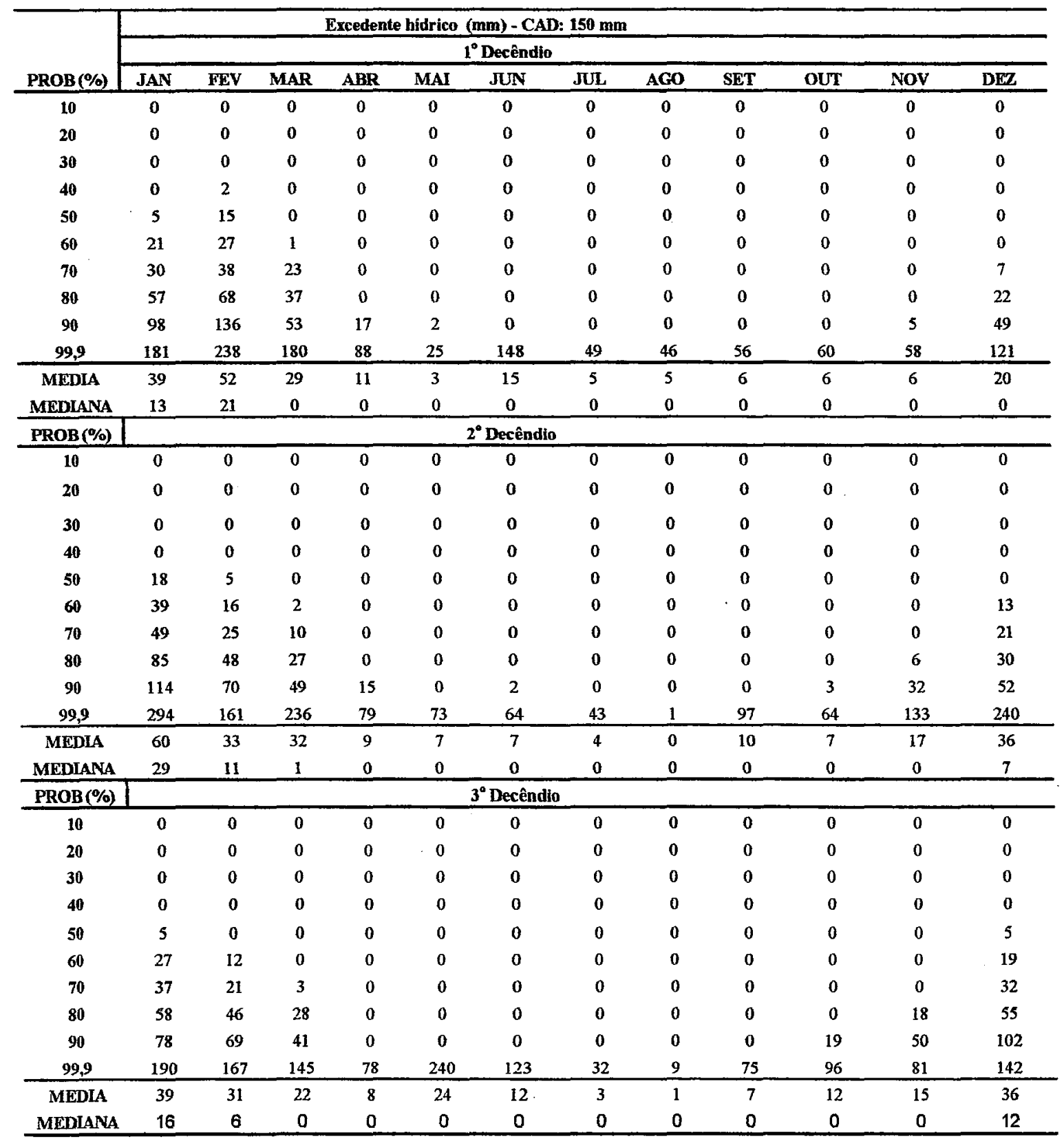


4.7 Uma aplicação agronômica das Tabelas de probabilidades dos componentes do balanço hídrico mensal

Objetivando exemplificar uma das formas de utilização das informações das tabelas de probabilidades dos componentes do balanço hídrico, foi escolhida a cultura do milho, considerando que a época do plantio ocorre em $1^{\circ}$ de setembro e a data da colheita em 30 de dezembro.

Os dados do Quadro 1 são referentes às probabilidade de $70 \%$ de ocorrência dos valores da evapotranspiração potencial e deficiência hídrica das Tabelas 13 e 26 e ao cálculo da queda de produtividade da cultura do milho.

Quadro 1- Quebra de produtividade e rendimento da cultura do milho, com probabilidades de $70 \%$ de ocorrências dos valores mensais da evapotranspiração potencial e deficiência hídrica.

\begin{tabular}{|c|c|c|c|c|c|c|c|}
\hline Mês & $\mathrm{CAD}(\mathrm{mm})$ & $\mathrm{ETP}(\mathbf{m m} / \mathbf{m e ̂ s})$ & $|\mathrm{DEF}(\mathrm{mm})|$ & $\begin{array}{c}\text { N.dias } \\
\text { (fases da cultura) }\end{array}$ & Ky & $\begin{array}{c}\text { Quebra da Produtividade (\%) } \\
\left(1-(\mathrm{Ya} / \mathrm{Ym})^{*} 100\right)\end{array}$ & $\begin{array}{c}\text { Rendimento } \\
(\mathrm{t} / \mathrm{ha})\end{array}$ \\
\hline SET & 50 & 78 & 41 & 60 & 0,4 & 12 & 8,8 \\
\hline$\overline{\text { OUT }}$ & 50 & 102 & 12 & & & & \\
\hline NOV & 100 & 111 & 2 & 30 & 1,5 & 16 & 7,4 \\
\hline$\overline{\mathrm{DEZ}}$ & 100 & 126 & $\begin{array}{lll}0 \\
\end{array}$ & 30 & 1,5 & 0 & 7,4 \\
\hline
\end{tabular}

Deve-se ressaltar que, nos dois primeiros meses, a profundidade do solo explorado pelas raízes corresponde a $\mathrm{CAD}$ de $50 \mathrm{~mm}$ e nos últimos meses a uma $\mathrm{CAD}$ de $100 \mathrm{~mm}$.

A metodologia utilizada foi proposta por Doorenbos \& Kassan (1979), conforme equação 13:

$$
\left(1-\frac{Y a}{Y m}\right)=K y\left(\frac{D E F}{E T P}\right)
$$

em que: $Y a=$ produção atual;

$\mathrm{Ym}=$ produção máxima (sem deficiência hídrica), considerada $10 \mathrm{t} / \mathrm{ha}$,

$\mathrm{Ky}=$ fator de sensibilidade à seca. 
Então, na primeira fase (60 dias) da cultura do milho, $\frac{Y a}{Y m}$ será:

$$
\begin{aligned}
& \frac{Y a}{Y m}=1-0,4 *\left(\frac{41+12}{78+102}\right) \\
& \frac{Y a}{Y m}=1-0,4 *\left(\frac{53}{180}\right) \\
& \frac{Y a}{Y m}=1-0,12 \\
& \frac{Y a}{Y m}=0,88
\end{aligned}
$$

sendo $\mathrm{Ym}=10 \mathrm{t} / \mathrm{ha}$ então,

$\mathrm{Ya}=0,88 * 10=8,8 \mathrm{t} / \mathrm{ha}$

Este resultado indica que esta fase promoverá uma queda de produção de $12 \% \mathrm{e}$, portanto, se não houver mais seca, a produção Ya será de 8,8t/ha, ou seja, a produção máxima daqui para frente não ultrapassará este valor.

Para a segunda fase fenológica (30 dias), encontram-se os valores da evapotranspiração potencial de $111 \mathrm{~mm}$ e deficiência hídrica de $12 \mathrm{~mm}$ nas tabelas $13 \mathrm{e}$ 26 , p. 20 e 36.

Tem-se, então:

$$
\begin{aligned}
& \left(1-\frac{Y a}{Y m}\right)=K y *\left(\frac{D E F}{E T P}\right) \\
& \left(1-\frac{Y a}{Y m}\right)=1,5 *\left(\frac{12}{111}\right) \\
& \frac{Y a}{Y m}=1-0,16 \\
& \frac{Y a}{Y m}=0,84
\end{aligned}
$$


sendo $\mathrm{Ym}=8,8 \mathrm{t} / \mathrm{ha}$, então,

$$
\begin{aligned}
& \mathrm{Ya}=0,84 * 8,8 \mathrm{t} / \mathrm{ha} \\
& \mathrm{Ya}=7,4 \mathrm{t} / \mathrm{ha}
\end{aligned}
$$

Este resultado indica que, em $70 \%$ dos casos, a produção do milho em Piracicaba será menor que 7,4 tha e que, se irrigada em $70 \%$ dos anos, conseguiria um acréscimo de $2,6 \mathrm{t} / \mathrm{ha}$, ou seja, $26 \%$ na produtividade.

Através dessa metodologia, tais tabelas podem ser usadas para se escolher a melhor época de plantio, para se estabelecer os efeitos do clima na produtividade e para se decidir sobre a viabilidade da irragação.

Outros estudos agronômicos e climáticos podem ser elaborados com o conteúdo apresentado nessas Tabelas. 


\section{5- CONCLUSÕES}

- O tipo climático de Piracicaba segundo o método de Köppen é Cfa com transição para Cwa.

- O tipo climático de Piracicaba segundo o método de Thornthwaite é $B_{1} r^{\prime} B^{\prime}{ }_{4}$ a' com transição para $\mathrm{C}_{2} \mathrm{r} \mathrm{B}_{4}{ }_{4} \mathrm{a}^{\prime}$.

-Apenas 10 anos de observação seria suficiente para identificar o clima de Piracicaba como ${ }_{1}$ r B' $_{4}$ a' (Thornthwaite) e como Cfa ou Cwa (Köppen).

- Existe uma certa tendência dos meses úmidos (DEZ, JAN e FEV) tornarem-se menos úmidos e osmeses secos (JUN, JL e AGO) tornarem-se menos secos, ou seja, um aumento das precipitações no inverno e diminuição no verão.

- O balanço hídrico normal, médio e mediano apresentou o mesmo tipo climático pelo método de Thorntwaite, $\mathrm{B}_{1} \mathrm{r}_{\mathrm{B}}{ }_{4}$ a', enquanto pelo método de Köppen o tipo foi Cfa para o balanço hídrico normal e médio e o tipo Cwa para o balanço hídrico mediano.

- As precipitações em Piracicaba não têm distribuição normal, sugerindo que a análise climática é mais representativa se forem utilizados valores medianos e não médios.

- O balanço hídrico elaborado com a temperatura e precipitação pluvial mediana difere do balanço hídrico mensal normal apresentado maior seca no período de inverno. 
- O estudo da freqüência e probabilidade de ocorrência dos elementos do clima e componentes do balanço hídrico permitem uma análise mais crítica do clima da região de Piracicaba.

- Para a temperatura, a média e a mediana apresenta-se muito próximas, indicando que este elemento pode ser utilizado numa análise climática com a mesma precisão nestas duas formas.

- O balanço hídrico normal decendial tem seus componentes iguais a $1 / 3$ do balanço hídrico normal mensal, indicando que uma análise climática tem a mesma precisão utilizando-se valores mensais ou decendiais.

- As probabilidades dos componentes do balanço hídrico para as diferentes CADs permitem análises agroclimáticas com relativa precisão. 
Anexo A

Quadro 1- Classificação climática de Köppen para a ordem de 10 anos (Período: 1917-1996)

\begin{tabular}{|c|c|c|c|c|c|c|c|}
\hline Ano & $T_{\text {mestatio }}$ & Verão & Inverno & $P_{\text {mestrumido }}$ & $\mathrm{P}_{\text {mist seco }}$ & PU/PS & $\mathbf{K}$ \\
\hline $1917-1926$ & 16,6 & 523 & 117 & 239 & 31 & 8 & Cfa \\
\hline $1918-1927$ & 16,6 & 518 & 123 & 230 & 31 & 7 & Cfa \\
\hline $1919-1928$ & 16,8 & 536 & 124 & 220 & 28 & 8 & $\mathrm{Cfa}$ \\
\hline $1920-1929$ & 16,7 & 603 & 107 & 243 & 29 & 8 & $\mathrm{Cfa}$ \\
\hline $1921-1930$ & 16,7 & 654 & 113 & 248 & 32 & 8 & Cfa \\
\hline $1922-1931$ & 16,9 & 679 & 115 & 253 & 35 & 7 & $\mathrm{Cfa}$ \\
\hline 1923-1932 & 17,0 & 676 & 107 & 237 & 32 & 7 & Cfa \\
\hline $1924-1933$ & 17,2 & 685 & 99 & 255 & 31 & 8 & Cfa \\
\hline $1925-1934$ & 17,2 & 708 & 101 & 273 & 30 & 9 & $\mathrm{Cfa}$ \\
\hline 1926-1935 & 17,6 & 723 & 99 & 276 & 32 & 9 & $\mathrm{Cfa}$ \\
\hline $1927-1936$ & 17,7 & 719 & 89 & 269 & 20 & 13 & Cwa \\
\hline 1928-1937 & 17,9 & 699 & 89 & 272 & 20 & 14 & Cwa \\
\hline $1929-1938$ & 18,0 & 679 & 86 & 266 & 18 & 15 & $\mathrm{Aw}$ \\
\hline $1930-1939$ & 17,7 & 649 & 87 & 262 & 19 & 14 & Cwa \\
\hline $1931-1940$ & 17,9 & 635 & 73 & 249 & 12 & 21 & Cwa \\
\hline $1932-1941$ & 17,7 & 607 & 75 & 252 & 10 & 25 & Cwa \\
\hline 1933-1942 & 17,4 & 598 & 77 & 239 & 15 & 16 & Cwa \\
\hline 1934.1943 & 17,4 & 623 & 73 & 245 & 15 & 16 & Cwa \\
\hline $1935-1944$ & 17,3 & 598 & 70 & 219 & 15 & 15 & Cwa \\
\hline $1936-1945$ & 17,2 & 626 & 84 & 217 & 16 & 14 & Cwa \\
\hline $1937-1946$ & 17,0 & 615 & 81 & 211 & 14 & 15 & Cwa \\
\hline $1938-1947$ & 16,8 & 668 & 83 & 233 & 14 & 17 & Cwa \\
\hline $1939-1948$ & 16,9 & 687 & 85 & 248 & 12 & 21 & Cwa \\
\hline $1940-1949$ & 17,1 & 688 & 82 & 241 & 13 & 19 & Cwa \\
\hline $1941-1950$ & 17,0 & 669 & 87 & 242 & 13 & 19 & Cwa \\
\hline $1942-1951$ & 16,8 & 675 & 84 & 249 & 14 & 18 & Cwa \\
\hline $1943-1952$ & 17,0 & 680 & 84 & 256 & 15 & 17 & Cwa \\
\hline 1944-1953 & 17,4 & 649 & 86 & 249 & 16 & 16 & Cwa \\
\hline 1945-1954 & 17,1 & 658 & 92 & 256 & 16 & 16 & Cwa \\
\hline $1946-1955$ & 17,2 & 637 & 83 & 251 & 24 & 10 & Cwa \\
\hline $1947-1956$ & 17,2 & 623 & 92 & 237 & 20 & 12 & Cwa \\
\hline 1948-1957 & 17,2 & 596 & 95 & 233 & 24 & 10 & Cwa \\
\hline $1949-1958$ & 17,1 & 587 & 97 & 231 & 22 & 11 & Cwa \\
\hline $1950-1959$ & 17,3 & 565 & 103 & 236 & 22 & 11 & Cwa \\
\hline $1951-1960$ & 17,2 & 598 & 104 & 250 & 21 & 12 & Cwa \\
\hline $1952-1961$ & 17,4 & 588 & 101 & 235 & 20 & 12 & Cwa \\
\hline $1953-1962$ & 17,2 & 589 & 102 & 228 & 21 & 11 & Cwa \\
\hline $1954-1963$ & 17,1 & 593 & 96 & 233 & 20 & 12 & Cwa \\
\hline $1955-1964$ & 16,9 & 589 & 100 & 221 & 27 & 8 & $\mathrm{Cfa}$ \\
\hline $1956-1965$ & 17,0 & 625 & 95 & 227 & 31 & 7 & $\mathrm{Cfa}$ \\
\hline $1957-1966$ & 17,3 & 659 & 80 & 246 & 27 & 9 & $\mathrm{Cfa}$ \\
\hline $1958-1967$ & 17,4 & 658 & 72 & 242 & 19 & 13 & Cwa \\
\hline $1959-1968$ & 17,3 & 663 & 73 & 244 & 19 & 13 & Cwa \\
\hline $1960-1969$ & 17,4 & 639 & 71 & 231 & 20 & 12 & Cwa \\
\hline $1961-1970$ & 17,4 & 607 & 76 & 220 & 22 & 10 & Cwa \\
\hline $1962-1971$ & 17,3 & 606 & 91 & 225 & 24 & 9 & $\mathrm{Cfa}$ \\
\hline
\end{tabular}


Continuação:

Quadro 1-Classificação climática de Köppen para a ordem de 10 anos (Período: 1917-1996)

\begin{tabular}{|c|c|c|c|c|c|c|c|}
\hline Ano & $T_{\text {mestritio }}$ & Verão & Invemo & $P_{\text {mestimido }}$ & $\mathbf{P}_{\text {méstseco }}$ & PU/PS & $\mathrm{K}$ \\
\hline 1963-1972 & 17,4 & 594 & 101 & 237 & 31 & 8 & $\mathrm{Cfa}$ \\
\hline $1964-1973$ & 17,3 & 572 & 112 & 206 & 32 & 6 & $\mathrm{Cfa}$ \\
\hline $1965-1974$ & 17,6 & 564 & 113 & 214 & 30 & 7 & $\mathrm{Cfa}$ \\
\hline $1966-1975$ & 17,5 & 558 & 111 & 205 & 34 & 6 & $\mathrm{Cfa}$ \\
\hline 1967.1976 & 17,1 & 568 & 129 & 203 & 37 & 5 & Cfa \\
\hline $1968-1977$ & 17,2 & 566 & 127 & 210 & 38 & 6 & $\mathrm{Cfa}$ \\
\hline $1969-1978$ & 17,3 & 532 & 131 & 195 & 36 & 5 & Cfa \\
\hline $1970-1979$ & 17,7 & 527 & 135 & 186 & 42 & 4 & $\mathrm{Cfa}$ \\
\hline $1971-1980$ & 16,9 & 510 & 126 & 190 & 33 & 6 & $\mathrm{Cfa}$ \\
\hline 1972-1981 & 16,8 & 522 & 114 & 192 & 30 & 6 & $\mathrm{Cfa}$ \\
\hline $1973-1982$ & 16,9 & 541 & 116 & 213 & 29 & 7 & $\mathrm{Cfa}$ \\
\hline $1974-1983$ & 16,9 & 573 & 125 & 207 & 28 & 7 & Cfa \\
\hline 1975-1984 & 17,3 & 558 & 126 & 201 & 29 & 7 & $\mathrm{Cfa}$ \\
\hline 1976-1985 & 17,2 & 507 & 123 & 198 & 25 & 8 & $\mathrm{Cfa}$ \\
\hline $1977-1986$ & 17,4 & 481 & 117 & 182 & 18 & 10 & Cwa \\
\hline $1978-1987$ & 17,3 & 495 & 123 & 184 & 19 & 10 & Cwa \\
\hline $1979-1988$ & 17,3 & 535 & 114 & 192 & 11 & 17 & Cwa \\
\hline $1980-1989$ & 17,4 & 587 & 119 & 217 & 17 & 13 & Cwa \\
\hline $1981-1990$ & 17,4 & 585 & 132 & 229 & 30 & 8 & $\mathrm{Cfa}$ \\
\hline 1982-1991 & 17,6 & 596 & 130 & 227 & 32 & 7 & $\mathrm{Cfa}$ \\
\hline 1983-1992 & 17,6 & 558 & 112 & 211 & 33 & 6 & $\mathrm{Cfa}$ \\
\hline 1984-1993 & 17,6 & 549 & 103 & 208 & 31 & 7 & Cfa \\
\hline $1985-1994$ & 17,5 & 572 & 98 & 212 & 33 & 6 & $\mathrm{Cfa}$ \\
\hline $1986-1995$ & 17,9 & 624 & 102 & 219 & 28 & 8 & $\mathrm{Cfa}$ \\
\hline $1987-1996$ & 17,9 & 649 & 92 & 236 & 17 & 14 & Cwa \\
\hline
\end{tabular}

Quadro 2- Classificação climática de Köppen para a ordem de 15 anos (Período: 1917-1996)

\begin{tabular}{|c|c|c|c|c|c|c|c|}
\hline Ano & Tmês+frio & Verato & Inverno & Pmês+úntido & Pmêstreco & PU/PS & $\mathrm{K}$ \\
\hline $1917-1931$ & 16,9 & 629 & 109 & 257 & 31 & 8 & Cfa \\
\hline $1918-1932$ & 17,1 & 629 & 112 & 251 & 31 & 8 & C fa \\
\hline 1919-1933 & 17.1 & 635 & 111 & 240 & 28 & 9 & $\mathrm{Cfa}$ \\
\hline $1920-1934$ & 17 & 653 & 98 & 237 & 28 & 8 & $\mathrm{Cfa}$ \\
\hline 1921-1935 & 17.1 & 650 & 100 & 237 & 26 & 9 & $\mathrm{Cfa}$ \\
\hline $1922-1936$ & 17,2 & 653 & 104 & 245 & 27 & 9 & C fa \\
\hline 1923-1937 & 17,3 & 633 & 101 & 247 & 25 & 10 & Cwa \\
\hline 1924-1938 & 17,5 & 633 & 93 & 253 & 26. & 10 & Cwa \\
\hline 1925-1939 & 17,4 & 554 & 97 & 255 & 27 & 9 & Cfa \\
\hline 1926-1940 & 17,6 & 690 & 91 & 257 & 25 & 10 & Cwa \\
\hline $1927-1941$ & 17,6 & 685 & 80 & 245 & 17 & 14 & Cwa \\
\hline $1928-1942$ & 17,6 & 683 & 81 & 252 & 20 & 13 & Cwa \\
\hline $1929-1943$ & 17,5 & 691 & 78 & 257 & 18 & 14 & Cwa \\
\hline $1930-1944$ & 17,5 & 649 & 73 & 248 & 18 & 14 & Cwa \\
\hline $1931-1945$ & 17,4 & 632 & 79 & 237 & 15 & 16 & Cwa \\
\hline $1932-1946$ & 17,3 & 609 & 83 & 232 & 19 & 12 & Cwa \\
\hline $1933-1947$ & 17.2 & 628 & 85 & 225 & 21 & 11 & Cwa \\
\hline $1934-1948$ & 17,3 & 634 & 83 & 212 & 24 & 9 & $\mathrm{Cfa}$ \\
\hline |1935-1949 & 17,3 & 641 & 84 & 218 & 24 & 9 & Cfa \\
\hline
\end{tabular}


Continuação:

Quadro 2- Classificação climática de Köppen para a ordem de 15 anos (Período: 1917-1996)

\begin{tabular}{|c|c|c|c|c|c|c|c|}
\hline Ano & Tmâstfrio & Verão & Inverno & Pmês+úmido & Pmêstseco & PU/PS & $\mathbf{K}$ \\
\hline $1936-1950$ & 17,2 & 656 & 83 & 227 & 22 & 10 & Cwa \\
\hline 1937-1951 & 17,1 & 656 & 77 & 233 & 18 & 13 & Cwa \\
\hline $1938-1952$ & 17 & 670 & 77 & 238 & 14 & 17 & Cwa \\
\hline 1939-1953 & 16,8 & 671 & 79 & 244 & 13 & 19 & Cwa \\
\hline $1940-1954$ & 17,1 & 656 & 78 & 238 & 13 & 18 & Cwa \\
\hline 1941-1955 & 17 & 633 & 86 & 242 & 18 & 13 & $\mathrm{Cwa}$ \\
\hline $1942-1956$ & 169 & 620 & 94 & 232 & 22 & 11 & Cwa \\
\hline $1943-1957$ & 17,1 & 626 & 97 & 243 & 25 & 10 & Cwa \\
\hline 1944-1958 & 17,1 & 610 & 100 & 241 & 25 & 10 & Cwa \\
\hline 1945-1959 & 17.3 & 619 & 105 & 251 & 28 & 9 & Cfa \\
\hline $1946-1960$ & 17,3 & 637 & 96 & 254 & 26 & 10 & Cwa \\
\hline $1947-1961$ & 17.4 & 637 & 91 & 249 & 21 & 12 & Cwa \\
\hline $1948-1962$ & 17,3 & 616 & 89 & 235 & 19 & 12 & $\mathrm{Cwa}$ \\
\hline $1949-1963$ & 17,3 & 609 & 87 & 234 & 16 & 15 & Cwa \\
\hline $1950-1964$ & 17,2 & 590 & 91 & 225 & 21 & 11 & Cwa \\
\hline 1951-1965 & 17,2 & 603 & 92 & 231 & 24 & 10 & Cwa \\
\hline $1952-1966$ & 17.3 & 609 & 90 & 229 & 24 & 10 & $\mathrm{Cwa}$ \\
\hline $1953-1967$ & 17,4 & 612 & 89 & 234 & 25 & 9 & Cfa \\
\hline 1954-1968 & 17.3 & 621 & 91 & 238 & 25 & 10 & Cwa \\
\hline $1955-1969$ & 17,3 & 606 & 91 & 234 & 25 & 9 & $\mathrm{Cfa}$ \\
\hline $1956-1970$ & 17.4 & 616 & 93 & 233 & 25 & 9 & $\mathrm{Cfa}$ \\
\hline 1957-1971 & 17.5 & 626 & 91 & 241 & 23 & 10 & $\mathrm{Cwa}$ \\
\hline $1958-1972$ & 17,5 & 616 & 94 & 238 & 27 & 9 & $\mathrm{Cfa}$ \\
\hline $1959-1973$ & 17,5 & 598 & 95 & 218 & 30 & 7 & $\mathrm{Cfa}$ \\
\hline 1960-1974 & 17,4 & 589 & 97 & 210 & 27 & 8 & Cfa \\
\hline 1961-1975 & 17.3 & 577 & 95 & 208 & 28 & 7 & $\mathrm{Cfa}$ \\
\hline $1962-1976$ & 17,1 & 595 & 109 & 210 & 31 & 7 & $\mathrm{Cfa}$ \\
\hline $1963-1977$ & 17,4 & 597 & 105 & 220 & 30 & 7 & Cfa \\
\hline $1964-1978$ & 17,3 & 581 & 114 & 209 & 29 & 7 & Cfa \\
\hline $1965-1979$ & 17.3 & 567 & 113 & 211 & 34 & 6 & $\mathrm{Cfa}$ \\
\hline $1966-1980$ & 17.3 & 540 & 113 & 199 & 35 & 6 & $C \mathrm{fa}$ \\
\hline $1967-1981$ & 17,1 & 532 & 114 & 201 & 33 & 6 & $\mathrm{Cfa}$ \\
\hline $1968-1982$ & 17.2 & 537 & 124 & 200 & 36 & 6 & $\mathrm{Cfa}$ \\
\hline $1969-1983$ & 17,3 & 538 & 131 & 193 & 34 & 6 & Cfa \\
\hline $1970-1984$ & 17,4 & 533 & 134 & 190 & 36 & 5 & $\mathrm{Cfa}$ \\
\hline $1971-1985$ & 17,2 & 509 & 127 & 183 & 34 & 5 & $\mathrm{Cfa}$ \\
\hline $1972-1986$ & 17,3 & 513 & 125 & 189 & 34 & 6 & $\mathrm{Cfa}$ \\
\hline $1973-1987$ & 17,1 & 529 & 120 & 202 & 25 & 8 & Cfa \\
\hline $1974-1988$ & 17,1 & 554 & 114 & 197 & 21 & 9 & Cfa \\
\hline $1975-1989$ & 17,2 & 582 & 117 & 212 & 27 & 8 & $\mathrm{Cfa}$ \\
\hline $1976-1990$ & 17,2 & 558 & 125 & 219 & 33 & 7 & $\mathrm{Cfa}$ \\
\hline $1977-1991$ & 17,4 & 552 & 116 & 217 & 29 & 7 & $\mathrm{Cfa}$ \\
\hline $1978-1992$ & 17,4 & 533 & 113 & 202 & 31 & 7 & $\mathrm{Cfa}$ \\
\hline 1979-1993 & 17.5 & 549 & 113 & 207 & 26 & 8 & Cfa \\
\hline $1980-1994$ & 17.7 & 563 & 110 & 208 & 26 & 8 & $\mathrm{Cfa}$ \\
\hline $1981-1995$ & 17.8 & 585 & 112 & 211 & 30 & 7 & Cfa \\
\hline $1982-1996$ & 17.9 & 599 & 110 & 213 & 30 & 7 & Cfa \\
\hline
\end{tabular}


Quadro 3- Classificação climática de Köppen para a ordem de 20 anos (Período: 1917-1996)

\begin{tabular}{|c|c|c|c|c|c|c|c|}
\hline Ano & Tmês+frio & Verão & Inverno & Pmês+úmido & Pmês+seco & PU/PS & $\mathrm{K}$ \\
\hline $1917-1936$ & 17.1 & 622 & 102 & 229 & 26 & 9 & $\mathrm{Cfa}$ \\
\hline 1918-1937 & 17,3 & 610 & 103 & 221 & 25 & 9 & $\mathrm{Cfa}$ \\
\hline 1919-1938 & 17,4 & 610 & 98 & 224 & 23 & 10 & $\mathrm{Cwa}$ \\
\hline $1920-1939$ & 17,2 & 627 & 92 & 232 & 24 & 10 & $\mathrm{Cwa}$ \\
\hline 1921-1940 & 17,3 & 646 & 89 & 233 & 22 & 11 & $\mathrm{Cwa}_{\mathrm{a}}$ \\
\hline $1922-1941$ & 17,3 & 645 & 90 & 234 & 23 & 10 & cwa \\
\hline 1923-1942 & 172 & 638 & 89 & 238 & 24 & 10 & Cwa \\
\hline $1924-1943$ & 17,3 & 655 & 84 & 250 & 24 & 10 & Cwa \\
\hline 1925-1944 & 17,3 & 655 & 82 & 246 & 24 & 10 & Cwa \\
\hline $1926-1945$ & 17,4 & 676 & 88 & 247 & 24 & 10 & $\mathrm{Cwa}$ \\
\hline $1927-1946$ & 17,4 & 669 & 84 & 233 & $\underline{22}$ & 11 & Cwa \\
\hline $1928-1947$ & 17.3 & 686 & 86 & 238 & 23 & 10 & Cwa \\
\hline 1929-1948 & 17,4 & 685 & 83 & 230 & 23 & 10 & Cwa \\
\hline $1930-1949$ & 17.4 & 671 & 84 & 236 & 22 & 11 & Cwa \\
\hline $1931-1950$ & 17,4 & 653 & 82 & 226 & 21 & 11 & $\mathrm{Cwa}$ \\
\hline $1932-1951$ & 17,3 & 642 & 80 & 223 & 19 & 12 & Cwa \\
\hline 1933-1952 & 17,2 & 639 & 83 & 214 & 19 & 11 & Cwa \\
\hline 1934-1953 & 17,2 & 637 & 83 & 212 & 20 & 11 & Cwa \\
\hline $1935-1954$ & 17,2 & 630 & 86 & 217 & 20 & 11 & $\mathrm{cwa}$ \\
\hline 1936-1955 & 17,2 & 633 & 84 & 226 & 20 & 11 & Cwa \\
\hline $1937-1956$ & 17,1 & 620 & 91 & 224 & 22 & 10 & Cwa \\
\hline 1938-1957 & 17 & 633 & 93 & 233 & 25 & 9 & Cfa \\
\hline $1939-1958$ & 17 & 638 & 98 & 240 & 26 & 9 & $\mathrm{Cfa}$ \\
\hline $1940-1959$ & 17.2 & 628 & 95 & 239 & 25 & 10 & $\mathrm{Cwa}_{\mathrm{w}}$ \\
\hline $1941-1960$ & 17,1 & 636 & 99 & 247 & 25 & 10 & Cwa \\
\hline $1942-1961$ & 17,2 & 633 & 96 & 243 & 24 & 10 & $\mathrm{Cwa}$ \\
\hline 1943-1962 & 17.2 & 636 & 96 & 243 & 23 & 11 & $\mathrm{Cwa}$ \\
\hline 1944-1963 & 17,3 & 623 & 95 & 242 & 22 & 11 & Cwa \\
\hline $1945-1964$ & 17,2 & 625 & 100 & 239 & 26 & 9 & $\mathrm{Cf} \mathrm{a}$ \\
\hline $1946-1965$ & 17,2 & 633 & 93 & 239 & 27 & 9 & Cfa \\
\hline $1947-1966$ & 17.3 & 643 & 89 & 242 & 23 & 11 & Cwa \\
\hline $1948-1967$ & 17,4 & 629 & 91 & 238 & 22 & 11 & Cwa \\
\hline 1949-1968 & 12,3 & 627 & 92 & 238 & 21 & 11 & Cwa \\
\hline $1950-1969$ & 17,3 & 604 & 92 & 234 & 21 & 11 & Cwa \\
\hline $1951-1970$ & 173 & 604 & 91 & 236 & 21 & 11 & Cwa \\
\hline 1952-1971 & 17.4 & 598 & 97 & 231 & 22 & 11 & Cwa \\
\hline $1953-1972$ & 17.4 & 593 & 99 & 233 & 29 & 8 & $\mathrm{Cfa}$ \\
\hline 1954-1973 & 17,5 & 583 & 102 & 220 & 31 & 7 & $\mathrm{Cfa}$ \\
\hline $1955-1974$ & 17,4 & 577 & 103 & 218 & 31 & 7 & $\mathrm{Cfa}$ \\
\hline $1956-1975$ & 17,3 & 593 & 104 & 213 & 31 & 7 & C f a \\
\hline $1957-1976$ & 17,3 & 615 & 104 & 225 & 31 & 7 & Cfa \\
\hline $1958-1977$ & 17.4 & 613 & 103 & 225 & 30 & 8 & $\mathrm{Cfa}$ \\
\hline 1959-1978 & 17,4 & 600 & 105 & 216 & 33 & 7 & C, a \\
\hline $1960-1979$ & 17.2 & 586 & 107 & 209 & 34 & 6 & $\mathrm{C} \mathrm{fa}$ \\
\hline $1961-1980$ & 17.2 & 559 & 106 & 198 & 34 & 6 & $\mathrm{Cfa}$ \\
\hline 1962-1981 & 17.1 & 564 & 110 & 206 & 34 & 6 & C f a \\
\hline $1963-1982$ & 17,3 & 570 & 116 & 212 & 35 & 6 & C.fa \\
\hline 1964-1983 & 17.3 & 575 & 127 & 207 & 36 & 6 & Cfa \\
\hline 1965-1984 & 17.5 & 562 & 122 & 208 & 32 & 7 & $\mathrm{Cfa}$ \\
\hline $1966-1985$ & 17,4 & 532 & 120 & 198 & 30 & 7 & Cfa \\
\hline |1967-1986 & 17,4 & 525 & 120 & 193 & 30 & 6 & C.fa \\
\hline
\end{tabular}


Continuação:

Quadro 3- Classificação climática de Köppen para a ordem de 20 anos (Período: 1917-1996)

\begin{tabular}{|c|c|c|c|c|c|c|c|}
\hline $\begin{array}{r}\text { Ano } \\
1968-1987 \\
\end{array}$ & $\begin{array}{r}\text { Tmês+frio } \\
17,4 \\
\end{array}$ & $\begin{array}{c}\text { Verão } \\
\mathbf{5 3 2}\end{array}$ & $\begin{array}{c}\text { Inverno } \\
121 \\
\end{array}$ & $\begin{array}{c}\text { Pmês+úmido } \\
197\end{array}$ & $\begin{array}{c}\text { Pmêstseco } \\
30 \\
\end{array}$ & $\begin{array}{c}\text { PU/PS } \\
7 \\
\end{array}$ & $\begin{array}{c}\mathrm{K} \\
\mathrm{Cfa}\end{array}$ \\
\hline $1969-1988$ & 17,3 & 534 & 120 & 190 & 29 & 7 & Cfa \\
\hline $1970-1989$ & 17.3 & 559 & 123 & 202 & 32 & 6 & $\mathrm{Cfa}$ \\
\hline $1971-1990$ & 17,2 & 548 & 128 & 202. & 35 & 6 & $\mathrm{Cfa}$ \\
\hline 1972-1991 & 17,3 & 560 & 124 & 207 & 35 & 6 & $\mathrm{Cfa}$ \\
\hline $1973-1992$ & 17,3 & 550 & 118 & 199 & 32 & 6 & $\mathrm{Cfa}$ \\
\hline $1974-1993$ & 17,4 & 562 & 117 & 208 & 30 & 7 & Cfa \\
\hline 1975-1994 & 17,5 & 567 & 115 & 207 & 31 & 7 & $\mathrm{Cfa}$ \\
\hline 1976-1995 & 17,5 & 567 & 117 & 209 & 32 & 7 & Cfa \\
\hline $1977-1996$ & 17,7 & 567 & 110 & 210 & 28 & 8 & $\mathrm{Cfa}$ \\
\hline
\end{tabular}

Quadro 4- Classificação climática de Köppen para a ordem de 25 anos (Periodo: 1917-1996)

\begin{tabular}{|c|c|c|c|c|c|c|c|}
\hline Ano & Tmês+firo & Verä́a & Invemo & Pmês+úmido & Pmês+seco & PU/PS & $\mathbf{K}$ \\
\hline $1917-1941$ & 17,2 & 620 & 94 & 223 & 23 & 10 & Cwa \\
\hline $1918-1942$ & 17,2 & 616 & 98 & 217 & 25 & 9 & $C f a$ \\
\hline $1919-1943$ & 17.2 & 630 & 96 & 227 & 22 & 10 & Cwa \\
\hline $1920-1944$ & 17,1 & 631 & 88 & 229 & 22 & 10 & Cwa \\
\hline 1921-1945 & 17,1 & 640 & 93 & 229 & 22 & 10 & Cwa \\
\hline $1922-1946$ & 17,2 & 637 & 96 & 225 & 25 & 9 & $\mathrm{Cf}_{\mathrm{f}}$ \\
\hline 1923-1947 & 17,1 & 648 & 94 & 230 & 26 & 9 & $\mathrm{Cfa}$ \\
\hline 1924-1948 & 173 & 655 & 90 & 229 & 26 & 9 & $\mathrm{Cfa}$ \\
\hline 1925-1949 & 17,3 & 668 & 91 & 236 & 26 & 9 & $\mathrm{Cfa}_{\mathrm{f}}$ \\
\hline $1926-1950$ & 17,4 & 681 & 90 & 235 & 26 & 9 & $\mathrm{Cfa}$ \\
\hline 1927-1951 & 17,3 & 681 & 82 & 229 & 22 & 10 & Cwa \\
\hline $1928-1952$ & 17,3 & 682 & 82 & 228 & 22 & 10 & Cwa \\
\hline 1929-1953 & 17,3 & 675 & 81 & 230 & 21 & 11 & Cwa \\
\hline $1930-1954$ & 17,3 & 653 & 82 & 218 & 21 & 10 & Cowa \\
\hline $1931-1955$ & 17,3 & 633 & 81 & 218 & 19 & 11 & Cwa \\
\hline $1932-1956$ & 173 & 615 & 86 & 212 & 19 & 11 & Cwa \\
\hline 1933-1957 & 17,2 & 615 & 89 & 212 & 23 & 9 & $\mathrm{Cfa}$ \\
\hline $1934-1958$ & 172 & 616 & 90 & 219 & 23 & 10 & Cwa \\
\hline $1935-1959$ & 17,3 & 611 & 92 & 221 & 23 & 10 & Cwa \\
\hline $1936-1960$ & 173 & 632 & 91 & 232 & 22 & 11 & Cwa \\
\hline $1937-1961$ & 17,3 & 629 & 87 & 234 & 21 & 11 & Cwa \\
\hline $1938-1962$ & 17,1 & 637 & 87 & 234 & 22 & 11 & Cwa \\
\hline $1939-1963$ & 17,1 & 641 & 85 & 240 & 23 & 10 & Cwa \\
\hline $1940-1964$ & 17,2 & 630 & 87 & 232 & 23 & 10 & Cwa \\
\hline $1941-1965$ & 17,1 & 630 & 90 & 236 & 23 & 10 & Cwa \\
\hline 1942-1966 & 17,2 & 635 & 87 & 237 & 24 & 10 & Cwa \\
\hline $1943-1967$ & 17,3 & 639 & 88 & 243 & 24 & 10 & Cwa \\
\hline $1944-1968$ & 17,3 & 632 & 89 & 243 & 25 & 10 & Cwa \\
\hline 1945-1969 & 173 & 627 & 91 & 243 & 25 & 10 & Cwa \\
\hline 1946-1970 & 17,3 & 626 & 88 & 241 & 25 & 10 & Cwa \\
\hline $1947-1971$ & 17,4 & 625 & 90 & 240 & 22 & 11 & Cwa \\
\hline 1948-1972 & 17,4 & 608 & 94 & 236 & 26 & 9 & Cfa \\
\hline $1949-1973$ & 17,3 & 594 & 96 & 223 & 27 & 8 & Cfa \\
\hline $1950-1974$ & 17.3 & 578 & 99 & 221 & 27 & 8 & C fa \\
\hline $1951-1975$ & 17,3 & 585 & 99 & 218 & 28 & 8 & $\mathrm{C}$ fa \\
\hline
\end{tabular}


Continuação:

Quadro 4- Classificação climática de Köppen para a ordem de 25 anos (Período: 1917-1996)

\begin{tabular}{|l|c|c|c|c|c|c|c|} 
Ano & Tmês+frio & Veräo & Inverno & Pmês+úmido & Pmês+seco & PU/PS & K \\
\hline $1952-1976$ & 17,3 & 593 & 105 & 219 & 31 & 7 & $\mathrm{Cfa}$ \\
\hline $1953-1977$ & 17,4 & 593 & 104 & 223 & 31 & 7 & $\mathrm{Cfa}$ \\
\hline $1954-1978$ & 17,4 & 586 & 106 & 218 & 33 & 7 & $\mathrm{Cfa}$ \\
\hline $1955-1979$ & 17,3 & 576 & 108 & 215 & 34 & 6 & $\mathrm{Cfa}$ \\
\hline $1956-1980$ & 17,3 & 574 & 105 & 210 & 33 & 6 & $\mathrm{Cfa}$ \\
\hline $1957-1981$ & 17,2 & 583 & 100 & 219 & 30 & 7 & $\mathrm{Cfa}$ \\
\hline $1958-1982$ & 17,3 & 586 & 102 & 217 & 29 & 7 & $\mathrm{Cfa}$ \\
\hline $1959-1983$ & 17,3 & 589 & 108 & 213 & 29 & 7 & $\mathrm{Cfa}$ \\
\hline $1960-1984$ & 17,4 & 577 & 108 & 207 & 30 & 7 & $\mathrm{Cfa}$ \\
\hline $1961-1985$ & 17,3 & 548 & 106 & 197 & 30 & 7 & $\mathrm{Cfa}$ \\
\hline $1962-1986$ & 17,3 & 549 & 112 & 197 & 30 & 7 & $\mathrm{Cfa}$ \\
\hline $1963-1987$ & 17,4 & 556 & 112 & 206 & 30 & 7 & $\mathrm{Cfa}$ \\
\hline $1964-1988$ & 17,4 & 561 & 113 & 201 & 30 & 7 & $\mathrm{Cfa}$ \\
\hline $1965-1989$ & 17,4 & 574 & 116 & 213 & 30 & 7 & $\mathrm{Cfa}$ \\
\hline $1966-1990$ & 17,4 & 558 & 120 & 211 & 34 & 6 & $\mathrm{Cfa}$ \\
\hline $1967-1991$ & 17,3 & 558 & 121 & 211 & 34 & 6 & $\mathrm{Cfa}$ \\
\hline $1968-1992$ & 17,3 & 546 & 118 & 205 & 35 & 6 & $\mathrm{Cfa}$ \\
\hline $1969-1993$ & 17,4 & 541 & 120 & 199 & 35 & 6 & $\mathrm{Cfa}$ \\
\hline $1970-1994$ & 17,5 & 549 & 118 & 199 & 35 & 6 & $\mathrm{Cfa}$ \\
\hline $1971-1995$ & 17,5 & 554 & 116 & 196 & 32 & 6 & $\mathrm{Cfaa}$ \\
\hline $1972-1996$ & 17,6 & 568 & 111 & 202 & 31 & 7 & $\mathrm{Cfa}$ \\
\hline
\end{tabular}

Quadro 5- Classificação climática de Köppen para a ordem de 30 anos (Período: 1917-1996)

\begin{tabular}{|c|c|c|c|c|c|c|c|}
\hline Ano & Tmês+frio & Veräo & Inverno & Pmês+úmido & Pmês+seco & PU/PS & $\mathrm{K}$ \\
\hline $1917-1946$ & 17.7 & 619 & 95 & 223 & 25 & 9 & $\mathrm{Cfa}$ \\
\hline $1918-1947$ & 17,1 & 629 & 99 . & 225 & 26 & 9 & Cfa \\
\hline $1919-1948$ & 17,2 & 636 & 99 & 226 & 26 & 9 & $\mathrm{Cfa}$ \\
\hline $1920-1949$ & 17,2 & 648 & 93 & 224 & 26 & 9 & $\mathrm{Cfa}$ \\
\hline 1921-1950 & 17,2 & 654 & 93 & 225 & 25 & 9 & $\mathrm{Cfa}$ \\
\hline 1922-1951 & 17,1 & 656 & 93 & 226 & 25 & 9 & $\mathrm{Cfa}$ \\
\hline 1923-1952 & 17,1 & 652 & 91 & 222 & 24 & 9 & $\mathrm{Cfa}$ \\
\hline $1924-1953$ & 17.2 & 654 & 87 & 223 & 24 & 9 & $\mathrm{Cfa}$ \\
\hline 1925-1954 & 17.2 & 657 & 89 & 223 & 24 & 9 & $\mathrm{Cfa}$ \\
\hline 1926-1955 & 17.3 & 663 & 90 & 228 & 24 & 10 & Cwa \\
\hline $1927-1956$ & 17.3 & 654 & 88 & 223 & 21 & 11 & Cwa \\
\hline 1928-1957 & 17,3 & 655 & 91 & 226 & 24 & 9 & $\mathrm{Cfa}$ \\
\hline 1929-1958 & 17.3 & 653 & 91 & 230 & 24 & 10 & Cwa \\
\hline $1930-1959$ & 17,4 & 636 & 91 & 222 & 23 & 10 & Cwa \\
\hline $1931-1960$ & 17.4 & 636 & 90 & 226 & 21 & 11 & Cwa \\
\hline $1932-1961$ & 17,4 & 624 & 89 & 219 & 20 & 11 & Cwa \\
\hline $1933-1962$ & 17,3 & 623 & 89 & 217 & 20 & 11 & $\mathrm{Cwa}$ \\
\hline 1934-1963 & 17.3 & 623 & 87 & 223 & 20 & 11 & $\mathrm{Cwa}$ \\
\hline $1935-1964$ & 17,3 & 616 & 88 & 218 & 22 & 10 & $\mathrm{Cwa}$ \\
\hline $1936-1965$ & 17.2 & 630 & 89 & 226 & 24 & 9 & C f a \\
\hline $1937-1966$ & 17.2 & 634 & 85 & 232 & 23 & 10 & $\mathrm{Cwa}$ \\
\hline 1938-1967 & 172 & 642 & 86 & 236 & 22 & 11 & $\mathrm{Cwa}$ \\
\hline 1939-1968 & 17.2 & 647 & 87 & 242 & 22 & 11 & Cwa \\
\hline $1940-1969$ & 17.3 & 632 & 87 & 237 & 23 & 10 & Cwa \\
\hline $1941-1970$ & 17.2 & 626 & 91 & 238 & 24 & 10 & $\mathrm{Cw}$ \\
\hline $1942-1971$ & 17,2 & 624 & 93 & 237 & 24 & 10 & $\mathrm{Cwa}$ \\
\hline
\end{tabular}


Continuação:

Quadro 5- Classificação climática de Köppen para a ordem de 30 anos (Período: 1917-1996)

\begin{tabular}{|c|c|c|c|c|c|c|c|}
\hline Ano & Tmêstfio & Veräo & Invemo & Pmês+úmido & Pmés+seco & PU/PS & K \\
\hline $1943-1972$ & 17,3 & 623 & 96 & 241 & 27 & 9 & Cfa \\
\hline $1944-1973$ & 17,3 & 606 & 99 & 230 & 28 & 8 & Cfa \\
\hline $1945-1974$ & 17,3 & 605 & 104 & 231 & 29 & 8 & Cfa \\
\hline $1946-1975$ & 17,3 & 608 & 98 & 226 & 29 & 8 & Cfa \\
\hline $1947-1976$ & 17,3 & 618 & 100 & 229 & 29 & 8 & Cfa \\
\hline $1948-1977$ & 17,4 & 607 & 99 & 228 & 28 & 8 & Cfa \\
\hline $1949-1978$ & 17,3 & 596 & 100 & 221 & 29 & 8 & Cfa \\
\hline $1950-1979$ & 17,3 & 579 & 103 & 218 & 30 & 7 & Cfa \\
\hline $1951-1980$ & 17,2 & 573 & 104 & 216 & 30 & 7 & Cfa \\
\hline $1952-1981$ & 17,3 & 572 & 103 & 216 & 29 & 7 & Cfa \\
\hline $1953-1982$ & 17,3 & 576 & 107 & 217 & 30 & 7 & Cfa \\
\hline $1954-1983$ & 17,3 & 581 & 111 & 216 & 30 & 7 & Cfa \\
\hline $1955-1984$ & 17,4 & 572 & 113 & 213 & 30 & 7 & Cfa \\
\hline $1956-1985$ & 17,3 & 564 & 111 & 208 & 30 & 7 & Cfa \\
\hline $1957-1986$ & 17,4 & 571 & 110 & 211 & 29 & 7 & Cfa \\
\hline $1958-1987$ & 17,4 & 574 & 110 & 212 & 26 & 8 & Cfa \\
\hline $1959-1988$ & 17,3 & 578 & 106 & 208 & 25 & 8 & Cfa \\
\hline $1960-1989$ & 17,3 & 587 & 109 & 212 & 28 & 8 & Cfa \\
\hline $1961-1990$ & 17,3 & 568 & 113 & 208 & 33 & 6 & Cfa \\
\hline $1962-1991$ & 17,3 & 574 & 113 & 213 & 33 & 6 & Cfa \\
\hline $1963-1992$ & 17,4 & 566 & 111 & 212 & 32 & 7 & Cfa \\
\hline $1964-1993$ & 17,4 & 565 & 114 & 207 & 34 & 6 & Cfa \\
\hline $1965-1994$ & 17,5 & 566 & 113 & 210 & 33 & 6 & Cfa \\
\hline $1966-1995$ & 17,6 & 574 & 113 & 205 & 33 & 6 & Cfa \\
\hline $1967-1996$ & 17,5 & 567 & 114 & 207 & 33 & 6 & Cfa \\
\hline
\end{tabular}

Quadro 6- Classificação climática de Köppen para a ordem de 35 anos (Período: 1917-1996)

\begin{tabular}{|c|c|c|c|c|c|c|c|}
\hline Ano & Tmês+frio & Veräo & Inverno & Pmês+úmido & Pmês+seco & PU/PS & K \\
\hline $1917-1951$ & 17,1 & 637 & 94 & 231 & 25 & 9 & $\mathrm{Cfa}$ \\
\hline $1918-1952$ & 17,1 & 637 & 95 & 229 & 24 & 10 & $\mathrm{Cwa}$ \\
\hline $1919-1953$ & 17,1 & 636 & 95 & 227 & 23 & 10 & $\mathrm{Cwa}$ \\
\hline $1920-1954$ & 17,1 & 640 & 90 & 225 & 23 & 10 & $\mathrm{Cwa}$ \\
\hline $1921-1955$ & 17,1 & 641 & 91 & 227 & 22 & 10 & $\mathrm{Cwa}$ \\
\hline $1922-1956$ & 17,2 & 635 & 96 & 221 & 24 & 9 & $\mathrm{Cfa}$ \\
\hline $1923-1957$ & 17,1 & 633 & 96 & 219 & 26 & 8 & $\mathrm{Cfa}$ \\
\hline $1924-1958$ & 17,2 & 636 & 94 & 221 & 26 & 9 & $\mathrm{Cfa}$ \\
\hline $1925-1959$ & 17,3 & 640 & 96 & 226 & 26 & 9 & $\mathrm{Cfa}$ \\
\hline $1926-1960$ & 17,3 & 659 & 94 & 233 & 25 & 9 & $\mathrm{Cfa}$ \\
\hline $1927-1961$ & 17,4 & 656 & 89 & 230 & 21 & 11 & $\mathrm{Cwa}$ \\
\hline $1928-1962$ & 17,4 & 656 & 89 & 228 & 22 & 10 & $\mathrm{Cwa}$ \\
\hline $1929-1963$ & 17,4 & 671 & 87 & 231 & 21 & 11 & $\mathrm{Cwa}$ \\
\hline $1930-1964$ & 17,3 & 636 & 89 & 219 & 23 & 10 & $\mathrm{Cwa}$ \\
\hline $1931-1965$ & 17,3 & 633 & 86 & 221 & 22 & 10 & $\mathrm{Cwa}$ \\
\hline $1932-1966$ & 17,3 & 629 & 85 & 219 & 21 & 10 & $\mathrm{Cwa}$ \\
\hline $1933-1967$ & 173 & 629 & 87 & 221 & 22 & 10 & $\mathrm{Cwa}$ \\
\hline $1934-1968$ & 17,3 & 631 & 86 & 227 & 22 & 10 & $\mathrm{Cwa}$ \\
\hline $1935-1969$ & 17,3 & 619 & 87 & 224 & 22 & 10 & $\mathrm{Cwa}$
\end{tabular}


Continuação:

Quadro 6- Classificação climática de Köppen para a ordem de 35 anos (Período: 1917-1996)

\begin{tabular}{|c|c|c|c|c|c|c|c|}
\hline Ano & Tmês+frio & Verão & Inverno & Pmês+únido & Pmês+seco & PU/PS & $\underline{K}$ \\
\hline 1936-1970 & 17,3 & 626 & 88 & 229 & 22 & 10 & Cwa \\
\hline $1937-1971$ & 17,3 & 623 & 89 & 232 & 22 & 11 & Cwa \\
\hline 1938-1972 & 17,2 & 626 & 92 & 235 & 26 & 9 & $\mathrm{Cfa}$ \\
\hline $1939-1973$ & 17.2 & 622 & 94 & 231 & 25 & 9 & $\mathrm{Cfa}$ \\
\hline $1940-1974$ & 17,3 & 612 & 95 & 227 & 25 & 9 & $\mathrm{Cfa}$ \\
\hline $1941-1975$ & 17,2 & 611 & 97 & 226 & 26 & 9 & $\mathrm{Cf}_{2}$ \\
\hline 1942-1976 & 17,1 & 618 & 100 & 228 & 27 & 8 & Cfa \\
\hline 1943-1977 & 17,3 & 619 & 100 & 233 & 28 & 8 & $\mathrm{Cfa}$ \\
\hline 1944-1978 & 17,3 & 605 & 102 & 227 & 28 & 8 & $\mathrm{Cf} \mathrm{a}$ \\
\hline $1945-1979$ & 17.3 & 600 & 105 & 227 & 30 & 8 & $\mathrm{Cfa}$ \\
\hline 1946-1980 & 17,3 & 594 & 100 & 222 & 31 & 7 & $\mathrm{Cfa}$ \\
\hline $1947-1981$ & 17,2 & 596 & 99 & 225 & 28 & 8 & C f a \\
\hline $1948-1982$ & 17,3 & 590 & 102 & 222 & 28 & 8 & $\mathrm{Cfa}$ \\
\hline 1949-1983 & 17.3 & 590 & 106 & 219 & 27 & 8 & $\mathrm{Cfa}$ \\
\hline $1950-1984$ & 17.4 & 575 & 108 & 216 & 27 & 8 & $\mathrm{Cfa}$ \\
\hline 1951-1985 & 17,3 & 565 & 107 & 213 & 27 & 8 & $\mathrm{Cfa}$ \\
\hline $1952-1986$ & 17,4 & 562 & 110 & 209 & 27 & 8 & $\mathrm{Cfa}$ \\
\hline 1953-1987 & 17,4 & 567 & 110 & 213 & 27 & 8 & $\mathrm{Cfa}$ \\
\hline 1954-1988 & 17,3 & 572 & 109 & 211 & 27 & 8 & $\mathrm{Cfa}$ \\
\hline $1955-1989$ & 17,3 & 580 & 112 & 216 & 29 & 7 & $\mathrm{Cfa}$ \\
\hline $1956-1990$ & 17,3 & 579 & 114 & 216 & 33 & 7 & $\mathrm{Cfa}$ \\
\hline 1957-1991 & 17.4 & 589 & 110 & 222 & 32 & 7 & $\mathrm{Cfa}$ \\
\hline 1958-1992 & 17,4 & 580 & 107 & 216 & 30 & 7 & $\mathrm{Cfa}$ \\
\hline $1959-1993$ & 17.4 & 579 & 107 & 212 & 30 & 7 & $\mathrm{Cfa}$ \\
\hline 1960-1994 & 17,4 & 576 & 107 & 209 & 31 & 7 & $\mathrm{Cfa}$ \\
\hline 1961-1995 & 17.5 & 572 & 106 & 204 & 31 & 7 & $\mathrm{Cfa}$ \\
\hline $1962-1996$ & 17,5 & 579 & 107 & 209 & 31 & 7 & $\mathrm{Cfa}$ \\
\hline
\end{tabular}

Legenda: Tmês+frio $=$ Temperatura do mês mais frio; Verão= Somatório $\left(\right.$ dez$\left.^{+}+\mathrm{jan}+\mathrm{fev}\right)$; Inverno= Somatório (jun+jul+ago); Pmêstúmido = Precipitação do mês mais úmido; Pmês+seco= Precipitação do mês mais seco; PU/PS= Pmês+úmido/Pmês+seco e K= Classificação climática de Köppen.

\section{Anexo B}

Quadro 1- Classificação climática de Thornthwaite para a ordem de 10 anos (Periodo: 1917-1996)

\begin{tabular}{|c|c|c|c|c|c|c|c|c|c|}
\hline Ano & EXC & DEF & EP & CV & Iu & Ia & Im & CVEP & TH \\
\hline $1917-1926$ & 156 & 21 & 1000 & 350 & 15,6 & 2,1 & 14 & 35,0 & $\mathrm{C}_{2} \mathrm{rB}_{4}^{\prime} \mathrm{a}^{\prime}$ \\
\hline $1918-1927$ & 177 & 22 & 1009 & 350 & 17,5 & 2,2 & 16 & 34,7 & $\mathrm{C}_{2} \mathrm{rB}_{4}^{\prime} \mathrm{a}^{\prime}$ \\
\hline $1919-1928$ & 175 & 26 & 1021 & 354 & 17,1 & 2,5 & 16 & 34,7 & $\mathrm{C}_{2} \mathrm{rB}_{4}^{\prime} \mathrm{a}^{\prime}$ \\
\hline $1920-1929$ & 227 & 29 & 1019 & 355 & 22,3 & 2,8 & 21 & 34,8 & $\mathrm{~B}_{1} \mathrm{rB}_{4}^{\prime} \mathrm{a}^{\prime}$ \\
\hline $1921-1930$ & 253 & 32 & 1024 & 354 & 24,7 & 3,1 & 23 & 34,6 & $\mathrm{~B}_{4} \mathrm{rB}_{4}^{\prime} \mathrm{a}^{\prime}$ \\
\hline $1922-1931$ & 325 & 22 & 1023 & 353 & 31,8 & 2,2 & 30 & 34,5 & $\mathrm{~B}_{4} \mathrm{rB}_{4}^{\prime} \mathrm{a}^{\prime}$ \\
\hline $1923-1932$ & 331 & 20 & 1025 & 353 & 32,3 & 2,0 & 31 & 34,4 & $\mathrm{~B}_{1} \mathrm{rB}_{4}^{\prime} \mathrm{a}^{\prime}$ \\
\hline $1924-1933$ & 297 & 29 & 1023 & 352 & 29,0 & 2,8 & 27 & 34,4 & $\mathrm{~B}_{1} \mathrm{rB}_{4}^{\prime} \mathrm{a}^{\prime}$ \\
\hline $1925-1934$ & 311 & 28 & 1029 & 355 & 30,2 & 2,7 & 29 & 34,5 & $\mathrm{~B}_{1} \mathrm{rB}_{4}^{\prime} \mathrm{a}^{\prime}$ \\
\hline $1926-1935$ & 336 & 25 & 1029 & 355 & 32,7 & 2,4 & 31 & 34,5 & $\mathrm{~B}_{1} \mathrm{rB}_{4}^{\prime} \mathrm{a}^{\prime}$ \\
\hline $1927-1936$ & 316 & 49 & 1039 & 358 & 30,4 & 4,7 & 28 & 34,5 & $\mathrm{~B}_{1} \mathrm{rB}_{4}^{\prime} \mathrm{a}^{\prime}$
\end{tabular}


Continuação:

Quadro 1- Classificação climática de Thornthwaite para a ordem de 10 anos (Período: 1917-1996)

\begin{tabular}{|c|c|c|c|c|c|c|c|c|c|}
\hline Ano & $\mathrm{EXC}$ & DEF & $\mathrm{EP}$ & $\mathrm{CV}$ & Iu & [a & Im & CVEP & $\mathrm{TH}$ \\
\hline $1928-1937$ & 293 & 32 & 1040 & 358 & 28,2 & 3,1 & 26 & 34,4 & $B_{1} \mathrm{rB}_{4}^{\prime} \mathrm{a}^{\prime}$ \\
\hline $1929-1938$ & 294 & 36 & 1042 & 359 & 28,2 & 3,5 & 26 & 34,5 & $B_{1} r^{\prime}{ }_{4}^{\prime} a^{\prime}$ \\
\hline $1930-1939$ & 284 & 32 & 1043 & 359 & 27,2 & 3,1 & 25 & 34,4 & $B_{1} \mathrm{BB}_{4}^{\prime} \mathrm{a}^{\prime}$ \\
\hline $1931-1940$ & 272 & 43 & 1049 & 361 & 25,9 & 4,1 & 23 & 34,4 & $\mathrm{~B}_{1} \mathrm{rB}_{4} \mathrm{a}^{\prime}$ \\
\hline $1932-1941$ & 239 & 48 & 1055 & 364 & 22,7 & 4,5 & 20 & 34,5 & $\mathrm{C}_{2} \times \mathrm{B}_{4}^{\prime} \mathrm{a}^{\prime}$ \\
\hline 1933-1942 & 225 & 47 & 1053 & 364 & 21,4 & 4,5 & 19 & 34,6 & $\mathrm{C}_{2} \mathrm{r} \mathrm{B}_{4}^{\prime} \mathrm{a}^{\prime}$ \\
\hline $1934 \cdot 1943$ & 296 & 52 & 1053 & 363 & 28,1 & 4,9 & 25 & 34,5 & $\mathrm{~B}_{1} \mathrm{rB}_{4}^{\prime} \mathrm{a}^{\prime}$ \\
\hline 1935-1944 & 305 & 52 & 1051 & 363 & 29,0 & 4,9 & 26 & 34,5 & $\mathrm{~B}_{1} \mathrm{IB}_{4} \mathrm{a}^{\prime}$ \\
\hline $1936-1945$ & 330 & 47 & 1050 & 359 & 31,4 & 4,5 & 29 & 34,2 & $B_{1} r^{\prime} B_{4} a^{\prime}$ \\
\hline $1937-1946$ & 321 & 50 & 1039 & 359 & 30,9 & 4,8 & 28 & 34,6 & $B_{1} r B_{4}^{\prime} a^{\prime}$ \\
\hline $1938-1947$ & 361 & 57 & 1035 & 359 & 34,9 & 5,5 & 32 & 34,7 & $B_{1} I B_{4}^{\prime} a^{\prime}$ \\
\hline $1939-1948$ & 382 & 66 & 1028 & 358 & 37,2 & 6,4 & 33 & 34,8 & $B_{1} \mathrm{rB}_{4}^{\prime} \mathrm{a}^{\prime}$ \\
\hline $1940-1949$ & 380 & 75 & 1023 & 355 & 37,1 & 7,3 & 33 & 34,7 & $B_{1} \mathrm{RB}_{4}^{\prime} \mathrm{a}^{\prime}$ \\
\hline $1941-1950$ & 387 & 64 & 1013 & 350 & 38,2 & 6,3 & 34 & 34,6 & $\mathrm{~B}_{1} \mathrm{SB}_{4}^{\prime} \mathrm{a}^{\prime}$ \\
\hline 1942-1951 & 402 & 81 & 1006 & 345 & 40,0 & 8,1 & 35 & 34,3 & $B_{1} r B_{4}^{\prime} a^{\prime}$ \\
\hline $1943-1952$ & 402 & 85 & 1003 & 343 & 40,1 & 8,5 & 35 & 34,2 & $B_{1} \mathrm{rB}_{4}^{\prime} \mathrm{a}^{\prime}$ \\
\hline $1944-1953$ & 337 & 80 & 1008 & 348 & 33,4 & 7,9 & 29 & 34,5 & $\mathrm{~B}_{1} \mathrm{rB}_{4}^{\prime} \mathrm{a}^{\prime}$ \\
\hline 1945-1954 & 315 & 68 & 1008 & 347 & 31,3 & 6,7 & 27 & 34,4 & $\mathrm{~B}_{1} \mathrm{rB}_{4}^{\prime} \mathrm{a}^{\prime}$ \\
\hline 1946-1955 & 287 & 74 & 1009 & 350 & 28,4 & 7,3 & 24 & 34,7 & $\mathrm{~B}_{1} \mathrm{rB}_{4}^{\prime} \mathrm{a}^{\prime}$ \\
\hline $1947-1956$ & 261 & 48 & 1005 & 352 & 26,0 & 4,8 & 23 & 35,0 & $\mathrm{~B}_{1} \mathrm{rB}_{4}^{\prime} \mathrm{a}^{\prime}$ \\
\hline $1948-1957$ & 231 & 43 & 1006 & 351 & 23,0 & 4,3 & 20 & 34,9 & $C_{2} \times B_{4}^{\prime} a^{\prime}$ \\
\hline 1949-1958 & 239 & 26 & 1006 & 352 & 23,8 & 2,6 & 22 & 35,0 & $\mathrm{~B}_{1} \mathrm{rB}_{4}^{\prime} \mathrm{a}^{\prime}$ \\
\hline $1950-1959$ & 226 & 23 & 1015 & 357 & 22,3 & 2,3 & 21 & 35,2 & $B_{1} \mathrm{rB}_{4}^{\prime} \mathrm{a}^{\prime}$ \\
\hline $1951-1960$ & 237 & 23 & 1015 & 357 & 23,3 & 2,3 & 22 & 35,2 & $B_{1} \mathrm{SB}_{4} \mathrm{a}^{\prime}$ \\
\hline $1952-1961$ & 213 & 34 & 1027 & 359 & 20,7 & 3,3 & 19 & 35,0 & $\mathrm{C}_{2}$ I B ${ }^{\prime} \mathrm{a}^{\prime}$ \\
\hline $1953-1962$ & 235 & 25 & 1025 & 360 & 22,9 & 2,4 & 21 & 35,1 & $\mathrm{~B}_{1} \mathrm{rB}_{4}^{\prime} \mathrm{a}^{\prime}$ \\
\hline $1954-1963$ & 223 & 36 & 1034 & 359 & 21,6 & 3,5 & 19 & 34,7 & $C_{2} \mp B^{\prime} a^{\prime}$ \\
\hline $1955-1964$ & 223 & 35 & 1024 & 355 & 21,8 & 3,4 & 20 & 34,7 & $\mathrm{C}_{2} \mathrm{r} \mathrm{B}_{4} \mathrm{a}^{\prime}$ \\
\hline $1956-1965$ & 258 & 39 & 1023 & 352 & 25,2 & 3,8 & 23 & 34,4 & $\mathrm{~B}_{1} \mathrm{rB}_{4} \mathrm{a}^{\prime}$ \\
\hline $1957-1966$ & 294 & 59 & 1030 & 353 & 28,5 & 5,7 & 25 & 34,3 & $B_{1} \mathrm{IB}_{4}^{\prime}+\mathrm{a}^{\prime}$ \\
\hline $1958-1967$ & 299 & 75 & 1037 & 352 & 28,8 & 7,2 & 24 & 33,9 & $B_{1} r^{\prime} A_{1} a^{\prime}$ \\
\hline 1959-1968 & 280 & 100 & 1033 & 348 & 27,1 & 9,7 & 21 & 33,7 & $B_{4} \mathrm{rB}_{4}^{\prime} \mathrm{a}^{\prime}$ \\
\hline $1960-1969$ & 251 & 96 & 1033 & 348 & 24,3 & 9,3 & 19 & 33,7 & $C_{2} \times B_{4}^{\prime} a^{\prime}$ \\
\hline $1961-1970$ & 221 & 82 & 1032 & 349 & 21,4 & 7,9 & 17 & 33,8 & $\mathrm{C}_{2} \times \mathrm{B}_{4}^{\prime} \mathrm{a}^{\prime}$ \\
\hline $1962-1971$ & 231 & 65 & 1032 & 357 & 22,4 & 6,3 & 19 & 34,6 & $C_{2} \times B_{4}^{\prime} a^{\prime}$ \\
\hline $1963-1972$ & 203 & 59 & 1028 & 357 & 19,7 & 5,7 & 16 & 34,7 & $\mathrm{C}_{2} \mathbf{r} \mathrm{B}_{4}^{\prime} \mathrm{a}^{\prime}$ \\
\hline $1964-1973$ & 195 & 35 & 1020 & 356 & 19,1 & 3,4 & 17 & 34,9 & $C_{2} \times B_{4}^{\prime} \mathrm{a}^{\prime}$ \\
\hline $1965-1974$ & 212 & 30 & 1020 & 358 & 20,8 & 2,9 & 19 & 35,1 & $C_{2} \times B_{4}^{\prime} a^{\prime}$ \\
\hline $1966-1975$ & 200 & 35 & 1026 & 360 & 19,5 & 3,4 & 17 & 35,1 & $\mathrm{C}_{2} \times \mathrm{B}_{4}^{\prime} \mathrm{B}^{\prime}$ \\
\hline $1967 \cdot 1976$ & 242 & 23 & 1016 & 356 & 23,8 & 2,3 & 22 & 35,0 & $\mathrm{~B}_{1} \mathrm{rB}_{4}^{\prime} \mathrm{a}^{\prime}$ \\
\hline $1968-1977$ & 236 & 16 & 1017 & 358 & 23,2 & 1,6 & 22 & 35,2 & $\mathrm{~B}_{1} \times \mathrm{B}_{4}^{\prime} \mathrm{a}^{\prime}$ \\
\hline $1969-1978$ & 215 & 12 & 1018 & 361 & 21,1 & 1,2 & 20 & 35,5 & $\mathrm{C}_{2} \times \mathrm{B}_{4}^{\prime} \mathrm{a}^{\prime}$ \\
\hline $1970-1979$ & 207 & 5 & 1012 & 357 & 20,5 & 0,5 & 20 & 35,3 & $C_{2} \times B_{4}^{\prime} a^{\prime}$ \\
\hline $1971-1980$ & 188 & 6 & 1001 & 351 & 18,8 & 0,6 & 18 & 35,1 & $\mathrm{C}_{2} \simeq \mathrm{B}_{4}^{\prime} \mathrm{a}^{\prime}$ \\
\hline $1972-1981$ & 198 & 13 & 995 & 344 & 19,9 & 1,3 & 19 & 34,6 & $\mathrm{C}_{2}$ \& $\mathrm{B}_{4}^{\prime} \mathrm{a}^{\prime}$ \\
\hline 1973-1982 & 244 & 15 & 1005 & 346 & 24,3 & 1,5 & 23 & 34,4 & $B_{1} r^{\prime} a_{4}^{\prime}$ \\
\hline $1974-1983$ & 342 & 11 & 1005 & 346 & 34,0 & 1,1 & 33 & 34,4 & $\mathrm{~B}_{1} \mathbf{r} \mathrm{B}_{4}^{\prime} \mathrm{a}^{\prime}$ \\
\hline $1975-1984$ & 292 & 7 & 1017 & 350 & 28,7 & 0,7 & 28 & 34,4 & $B_{3} \mathrm{rB}_{4}^{\prime} \mathrm{a}^{\prime}$ \\
\hline $1976-1985$ & 249 & 7 & 1019 & 352 & 24,4 & 0,7 & 24 & 34,5 & $B_{1} r^{\prime} a_{a}^{\prime}$ \\
\hline $1977-1986$ & 206 & 8 & 1036 & 355 & 19,9 & 0,8 & 19 & 34,3 & $C_{2} \Gamma B_{4}^{\prime} a^{\prime}$ \\
\hline $1978-1987$ & 230 & 8 & 1035 & 357 & 22,2 & 0,8 & 22 & 34,5 & $\mathrm{~B}_{1} \mathrm{IB}_{4}^{{ }_{4} \mathrm{a}^{\prime}}$ \\
\hline $1979-1988$ & 284 & 12 & 1045 & 360 & 27,2 & 1,1 & 26 & 34,4 & $\mathrm{~B}_{1} \mathrm{rB}_{4}^{\prime} \mathrm{a}^{\prime}$ \\
\hline 1980-1989 & 328 & 14 & 1047 & 363 & 31,3 & 1,3 & 31 & 34,7 & $B_{1} \mathrm{rB}_{4}^{\prime} \mathrm{a}^{\prime}$ \\
\hline 1981.1990 & 331 & 7 & 1063 & 371 & 31,1 & 0,7 & 31 & 34,9 & $B_{1} r B_{1}^{\prime} a^{\prime}$ \\
\hline $1982-1991$ & 353 & 5 & 1070 & 375 & 33,0 & 0,5 & 33 & 35,0 & $B_{1} \mathrm{IB}_{4}^{\prime} \mathrm{a}^{\prime}$ \\
\hline 1983.1992 & 310 & 5 & 1071 & 376 & 28,9 & 0,5 & 29 & 35,1 & $\mathrm{~B}_{1} \mathrm{rB}_{4}^{\prime} \mathrm{g}^{\prime}$ \\
\hline $1984-1993$ & 239 & 11 & 1078 & 376 & 22,2 & 1,0 & 22 & 34,9 & $B_{1} R^{\prime} a^{\prime}$ \\
\hline $1985-1994$ & 291 & 14 & 1078 & 376 & 27,0 & 1,3 & 26 & 34,9 & $\mathrm{~B}_{1} \mathrm{~B}_{4}^{\prime} \mathrm{a}^{\prime}$ \\
\hline $1986-1995$ & 358 & 16 & 1081 & 379 & 33,1 & 1,5 & 32 & 35,1 & $B_{1} \mathrm{r}^{\prime} \mathrm{a}^{\prime}$ \\
\hline $1987-1996$ & 376 & 16 & 1083 & 381 & 34,7 & 1,5 & 34 & 35.2 & $\mathrm{~B}_{1} \mathrm{rB}_{4}^{\prime} \mathrm{a}^{\prime}$ \\
\hline
\end{tabular}


Quadro 2- Classificação climática de Thornthwaite para a ordem de 15 anos (Período: 1917-1996)

\begin{tabular}{|c|c|c|c|c|c|c|c|c|c|}
\hline Ano & EXC & DEF & EP & $\mathrm{CV}$ & Iu & Ie & Im & CVEP & TH \\
\hline $1917-1931$ & 251 & 25 & 1009 & 351 & 24,88 & 2,48 & 23 & 34,79 & $\mathrm{~B}_{1} \simeq \mathrm{B}_{4}^{\prime} \mathrm{a}^{t}$ \\
\hline $1918-1932$ & 262 & 25 & 1018 & 352 & 25,74 & 2,46 & 24 & 34,58 & $\mathrm{~B}_{1} \mathrm{rB}_{4}^{\prime} \mathrm{a}^{\prime}$ \\
\hline $1919-1933$ & 252 & 26 & 1019 & 354 & 24,73 & 2,55 & 23 & 34,74 & $B_{1} r B_{4}^{\prime} a^{\prime}$ \\
\hline $1920-1934$ & 261 & 36 & 1021 & 353 & 25,56 & 3,53 & 23 & 34,57 & $\mathrm{~B}_{1} \pm \mathrm{B}_{4}^{\prime} \mathrm{a}^{\prime}$ \\
\hline $1921-1935$ & 257 & 30 & 1024 & 356 & 25,10 & 2,93 & 23 & 34,77 & $B_{1} r B_{4}^{\prime} a^{\prime}$ \\
\hline $1922-1936$ & 276 & 31 & 1032 & 356 & 26,74 & 3,00 & 25 & 34,50 & $\mathrm{~B}_{1} \times B_{4}^{\prime} \mathrm{a}^{\prime}$ \\
\hline $1923-1937$ & 270 & 22 & 1032 & 356 & 26,16 & 2,13 & 25 & 34,50 & $\mathrm{~B}_{1} \mathrm{r} \mathrm{B}_{4}^{\prime} \mathrm{a}^{\prime}$ \\
\hline $1924-1938$ & 252 & 26 & 1037 & 356 & 24,30 & 2,51 & 23 & 34,33 & $\mathrm{~B}_{1} \times \mathrm{B}_{4}^{\prime} \mathrm{a}^{\prime}$ \\
\hline $1925-1939$ & 284 & 20 & 1040 & 359 & 27,31 & 1,92 & 26 & 34,52 & $B_{1} r B_{4}^{\prime} a^{\prime}$ \\
\hline $1926-1940$ & 309 & 27 & 1044 & 359 & 29,60 & 2,59 & 28 & 34,39 & $\mathrm{~B}_{4} \mathrm{rB}_{4} \mathrm{a}^{\prime}$ \\
\hline $1927-1941$ & 306 & 43 & 1046 & 361 & 29,25 & 4,11 & 27 & 34,51 & $\mathrm{~B}_{1} \Gamma \mathrm{B}_{4} \mathrm{a}^{\prime}$ \\
\hline $1928-1942$ & 292 & 38 & 1046 & 363 & 27,92 & 3,63 & 26 & 34,70 & $B_{1} \times B_{4}^{\prime} a^{\prime}$ \\
\hline $1929-1943$ & 332 & 42 & 1040 & 360 & 31,92 & 4,04 & 30 & 34,62 & $\mathrm{~B}_{1}\left\ulcorner\mathrm{~B}_{1}^{\prime} \mathrm{a}^{\prime}\right.$ \\
\hline $1930-1944$ & 313 & 52 & 1042 & 360 & 30,04 & 4,99 & 27 & 34,55 & $\mathrm{~B}_{1} \times \mathrm{B}_{4}^{\prime} \mathrm{a}^{\prime}$ \\
\hline $1931-1945$ & 310 & 43 & 1041 & 359 & 29,78 & 4,13 & 27 & 34,49 & $B_{1} \simeq B_{4}^{\prime} a^{\prime}$ \\
\hline $1932-1946$ & 272 & 50 & 1043 & 360 & 26,08 & 4,79 & 23 & 34,52 & $\mathrm{~B}_{1} \simeq \mathrm{B}_{4}^{\prime} \mathrm{a}^{\prime}$ \\
\hline $1933-1947$ & 290 & 48 & 1038 & 359 & 27,94 & 4,62 & 25 & 34,59 & $\mathrm{~B}_{1} \mathrm{rB}_{4}^{\prime} \mathrm{a}^{\prime}$ \\
\hline $1934-1948$ & 310 & 51 & 1040 & 360 & 29,81 & 4,90 & 27 & 34,62 & $\mathrm{~B}_{1} \times \mathrm{B}_{4} \mathrm{a}^{\prime}$ \\
\hline $1935-1949$ & 326 & 47 & 1035 & 359 & 31,50 & 4,54 & 29 & 34,69 & $\mathrm{~B}_{1} \times \mathrm{B}_{4}^{\prime} \mathrm{a}^{\prime}$ \\
\hline $1936-1950$ & 348 & 58 & 1033 & 355 & 33,69 & 5,61 & 30 & 34,37 & $\mathrm{~B}_{1} \times \mathrm{B}_{4}^{\prime} \mathrm{a}^{\prime}$ \\
\hline $1937-1951$ & 363 & 63 & 1022 & 352 & 35,52 & 6,16 & 32 & 34,44 & $\mathrm{~B}_{1} \mathrm{rB}_{4}^{\prime} \mathrm{a}^{\prime}$ \\
\hline $1938-1952$ & 363 & 72 & 1021 & 353 & 35,55 & 7,05 & 31 & 34,57 & $B_{1} \times B_{4}^{\prime} a^{\prime}$ \\
\hline $1939-1953$ & 362 & 74 & 1017 & 351 & 35,59 & 7,28 & 31 & 34,51 & $\mathrm{~B}_{1} \mathrm{rB}_{4}^{\prime} \mathrm{a}^{\prime}$ \\
\hline $1940-1954$ & 336 & 78 & 1020 & 352 & 32,94 & 7,65 & 28 & 34,51 & $\mathrm{~B}_{1} \times \mathrm{B}_{4}^{\prime} \mathrm{a}^{\prime}$ \\
\hline $1941-1955$ & 320 & 64 & 1014 & 351 & 31,56 & 6,31 & 28 & 34,62 & $\mathrm{~B}_{1} \times \mathrm{B}_{4}^{\prime} \mathrm{a}^{\prime}$ \\
\hline $1942-1956$ & 299 & 56 & 1010 & 351 & 29,60 & 5,54 & 26 & 34,75 & $\mathrm{~B}_{1} \times \mathrm{B}_{4}^{\prime} \mathrm{a}^{\prime}$ \\
\hline 1943-1957 & 299 & 50 & 1007 & 350 & 29,69 & 4,97 & 27 & 34,76 & $\mathrm{~B}_{1} \times \mathrm{B}_{4}^{\prime} \mathrm{a}^{\prime}$ \\
\hline $1944-1958$ & 273 & 36 & 1010 & 352 & 27,03 & 3,56 & 25 & 34,85 & $\mathrm{~B}_{1} \times \mathrm{B}_{4}^{\prime} \mathrm{a}^{\prime}$ \\
\hline $1945-1959$ & 278 & 31 & 1010 & 354 & 27,52 & 3,07 & 26 & 35,05 & $\mathrm{~B}_{1} \times \mathrm{B}_{4}^{\prime} \mathrm{a}^{\prime}$ \\
\hline $1946-1960$ & 281 & 39 & 1011 & 353 & 27,79 & 3,86 & 25 & 34,92 & $B_{1} \times B_{4}^{\prime} a^{\prime}$ \\
\hline $1947-1961$ & 279 & 40 & 1014 & 352 & 27,51 & 3,94 & 25 & 34,71 & $\mathrm{~B}_{1} \times \mathrm{B}_{4}^{\prime} \mathrm{a}^{\prime}$ \\
\hline $1948-1962$ & 272 & 44 & 1014 & 352 & 26,82 & 4,34 & 24 & 34,71 & $\mathrm{~B}_{1} \times \mathrm{B}_{4}^{\prime} \mathrm{a}^{\prime}$ \\
\hline $1949-1963$ & 254 & 52 & 1021 & 352 & 24,88 & 5,09 & 22 & 34,48 & $B_{1}+B_{4}^{\prime} a^{\prime}$ \\
\hline $1950-1964$ & 229 & 50 & 1022 & 354 & 22,41 & 4,89 & 19 & 34,64 & $\mathrm{C}_{2} \times \mathrm{B}_{4}^{\prime} \mathrm{a}^{\prime}$ \\
\hline $1951-1965$ & 233 & 47 & 1021 & 353 & 22,82 & 4,60 & 20 & 34,57 & $\mathrm{C}_{2} \times \mathrm{B}_{4}^{4} \mathrm{a}^{\prime}$ \\
\hline $1952-1966$ & 223 & 42 & 1026 & 357 & 21,73 & 4,09 & 19 & 34,80 & $\mathrm{C}_{2} \mathrm{rB_{4 } ^ { \prime } \mathrm { a } ^ { \prime }}$ \\
\hline $1953-1967$ & 234 & 46. & 1029 & 356 & 22,74 & 4,47 & 20 & 34,60 & $\mathrm{C}_{2} \times \mathrm{B}_{4}^{\prime} \mathrm{a}^{\mathrm{t}}$ \\
\hline $1954-1968$ & 235 & 50 & 1027 & 355 & 22,88 & 4,87 & 20 & 34,57 & $\mathrm{C}_{2} \times \mathrm{B}_{4}^{\prime} \mathrm{a}^{\prime}$ \\
\hline $1955-1969$ & 232 & 47 & 1026 & 354 & 22,61 & 4,58 & 20 & 34,50 & $\mathrm{C}_{2} r \mathrm{~B}_{4}^{\prime} \mathrm{a}^{\prime}$ \\
\hline $1956-1970$ & 237 & 43 & 1026 & 354 & 23,10 & 4,19 & 21 & 34,50 & $\mathbf{B}_{1} \times B_{4}^{\prime} \mathbf{a}^{\prime}$ \\
\hline $1957-1971$ & 256 & 51 & 1032 & 355 & 24,81 & 4,94 & 22 & 34,40 & $B_{1} r B_{4}^{\prime} a^{\prime}$ \\
\hline $1958-1972$ & 247 & 49 & 1030 & 355 & 23,98 & 4,76 & 21 & 34,47 & $\mathrm{~B}_{1} \times \mathrm{B}_{4}^{\prime} \mathrm{a}^{\prime}$ \\
\hline $1959-1973$ & 225 & 59 & 1030 & 354 & 21,84 & 5,73 & 18 & 34,37 & $\mathrm{C}_{2} \times \mathrm{B}_{4}^{\prime} \mathrm{a}^{\prime}$ \\
\hline $1960-1974$ & 221 & 56 & 1025 & 353 & 21,56 & 5,46 & 18 & 34,44 & 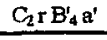 \\
\hline $1961-1975$ & 213 & 59 & 1028 & 355 & 20,72 & 5,74 & 17 & 34,53 & $\mathrm{C}_{2} \mathrm{rB}_{4}^{\prime} \mathrm{a}^{\prime}$ \\
\hline $1962-1976$ & 250 & 43 & 1019 & 355 & 24,53 & 4,22 & 22 & 34,84 & $\mathrm{~B}_{1} \times \mathrm{B}_{4}^{\prime} \mathrm{a}^{\prime}$ \\
\hline $1963-1977$ & 233 & 41 & 1025 & 356 & 22,73 & 4,00 & 20 & 34,73 & $\mathrm{C}_{2} \times \mathrm{B}_{4}^{\prime} \mathrm{a}^{\prime}$ \\
\hline $1964-1978$ & 222 & 27 & 1017 & 355 & 21,83 & 2,65 & 20 & 34,91 & $\mathrm{C}_{2} \times \mathrm{B}_{4}^{\prime} \mathrm{a}^{\prime}$ \\
\hline $1965-1979$ & 216 & 21 & 1016 & 355 & 21,26 & 2,07 & 20 & 34,94 & $\mathrm{C}_{2} \times \mathrm{B}_{4}^{\prime} \mathrm{a}^{\prime}$ \\
\hline $1966-1980$ & 186 & 18 & 1011 & 353 & 18,40 & 1,78 & 17 & 34,92 & $C_{2} \times B_{4}^{\prime} a^{\prime}$ \\
\hline $1967-1981$ & 193 & 20 & 1009 & 350 & 19,13 & 1,98 & 18 & 34,69 & $C_{2} \times B_{4}^{\prime} a^{\prime}$ \\
\hline
\end{tabular}


Continuação:

Quadro 2- Classificação climática de Thornthwaite para a ordem de 15 anos (Periodo: 1917-1996)

\begin{tabular}{|c|c|c|c|c|c|c|c|c|c|}
\hline Ano & EXC & DEF & $\mathrm{EP}$ & $\mathrm{CV}$ & Iu & $\mathrm{Ia}$ & Im & CVEP & TH \\
\hline $1968-1982$ & 217 & 12 & 1008 & 351 & 21,53 & 1,19 & 21 & 34,82 & $\mathrm{~B}_{1} \mathrm{rB} \mathrm{B}_{4}^{\prime} \mathrm{E}^{\prime}$ \\
\hline $1969-1983$ & 276 & 6 & 1011 & 352 & 27,30 & 0,59 & 27 & 34,82 & $B_{1} r B_{4}^{\prime} a^{\prime}$ \\
\hline $1970-1984$ & 258 & 3 & 1016 & 354 & 25,39 & 0,30 & 25 & 34,84 & $B_{1} \mathrm{rB}_{4}^{\prime} \mathrm{a}^{\prime}$ \\
\hline $1971-1985$ & 229 & 5 & 1019 & 355 & 22,47 & 0,49 & 22 & 34,84 & $B_{1} r B_{4}^{\prime} a^{\prime}$ \\
\hline $1972-1986$ & 232 & 5 & 1021 & 354 & 22,72 & 0,49 & 22 & 34,67 & $\mathrm{~B}_{1} \mathrm{rB}_{4}^{\prime} \mathrm{a}^{\prime}$ \\
\hline $1973-1987$ & 248 & 8 & 1030 & 357 & 24,08 & 0,78 & 24 & 34,66 & $B_{1} r B_{4}^{\prime} a^{\prime}$ \\
\hline $1974-1988$ & 280 & 11 & 1031 & 358 & 27,16 & 1,07 & 27 & 34,72 & $B_{1} ז B_{4}^{\prime} a^{\prime}$ \\
\hline 1975-1989 & 290 & 7 & 1033 & 357 & 28,07 & 0,68 & 28 & 34,56 & $B_{1} \times B_{4}^{\prime} a^{\prime}$ \\
\hline $1976-1990$ & 278 & 4 & 1036 & 361 & 26,83 & 0,39 & 27 & 34,85 & $B_{1} \times B_{4}^{\prime} a^{\prime}$ \\
\hline $1977-1991$ & 269 & 8 & 1044 & 362 & 25,77 & 0,77 & 25 & 34,67 & $\mathrm{~B}_{1} \mathrm{rB}_{4}^{\prime} \mathrm{a}^{4}$ \\
\hline $1978-1992$ & 273 & 7 & 1042 & 362 & 26,20 & 0,67 & 26 & 34,74 & $B_{1} \Gamma E_{4}^{\prime} a^{\prime}$ \\
\hline 1979-1993 & 295 & 6 & 1050 & 363 & 28,10 & 0,57 & 28 & 34,57 & $\mathrm{~B}_{1} \mathrm{r} \mathrm{B}_{4}^{\prime} \mathrm{a}^{\prime}$ \\
\hline $1980-1994$ & 340 & 12 & 1035 & 334 & 32,85 & 1,16 & 32 & 32,27 & $B_{1} \times B_{4}^{1} a^{1}$ \\
\hline $1981-1995$ & 342 & 11 & 1074 & 374 & 31,84 & 1,02 & 31 & 34,82 & $B_{1} r B_{4}^{\prime} a^{\prime}$ \\
\hline $1982-1996$ & 352 & 8 & 1079 & 377 & 32,62 & 0,74 & 32 & 34,94 & $B_{1} r^{\prime} a_{4}^{\prime}$ \\
\hline
\end{tabular}

Quadro 3- Classificação climática de Thornthwaite para a ordem de 20 anos (Período: 1917-1996)

\begin{tabular}{|c|c|c|c|c|c|c|c|c|c|}
\hline Ano & EXC & DEF & EP & $\mathrm{CV}$ & Iu & Ia & Im & CVEP & $\mathrm{TH}$ \\
\hline $1917-1936$ & 231 & 32 & 1018 & 354 & 23 & 3 & 21 & 34,8 & $B_{1} \Gamma B_{4}^{\prime} a^{\prime}$ \\
\hline 1918-1937 & 232 & 28 & 1024 & 352 & 23 & 3 & 21 & 34,4 & $\mathrm{~B}_{1} \mathrm{rB}_{4}^{\prime} \mathrm{a}^{\prime}$ \\
\hline 19]9-1938 & 227 & 28 & 1030 & 355 & 22 & 3 & 20 & 34,5 & $\mathrm{C}_{2} \times \mathrm{B}_{4}^{\prime} \mathrm{a}^{\prime}$ \\
\hline 1920-1939 & 248 & 29 & 1030 & 357 & 24 & 3 & 22 & 34,7 & $B_{1} \Gamma B_{4}^{\prime} a^{\prime}$ \\
\hline $1921-1940$ & 253 & 33 & 1036 & 358 & 24 & 3 & 23 & 34,6 & $B_{1} \simeq B_{4}^{\prime} a^{\prime}$ \\
\hline $1922-1941$ & 275 & 33 & 1038 & 359 & 26 & 3 & 25 & 34,6 & $\mathrm{~B}_{1} \times \mathrm{B}_{4} \mathrm{a}^{\prime}$ \\
\hline 1923-1942 & 274 & 31 & 1038 & 359 & 26 & 3 & 25 & 34,6 & $\mathrm{~B}_{1} \times \mathrm{B}_{4}^{\prime} \mathrm{a}^{\prime}$ \\
\hline $1924-1943$ & 291 & 37 & 1038 & 359 & 28 & 4 & 26 & 34,6 & $B_{1} \times B_{4}^{\prime} a^{\prime}$ \\
\hline $1925-1944$ & 302 & 40 & 1040 & 360 & 29 & 4 & 27 & 34,6 & $B_{1} \times B_{4}^{\prime} a^{\prime}$ \\
\hline $1926-1945$ & 329 & 34 & 1038 & 357 & 32 & 3 & 30 & 34,4 & $B_{1} \tau B_{4}^{\prime} a^{\prime}$ \\
\hline $1927-1946$ & 311 & 44 & 1039 & 360 & 30 & 4 & 27 & 34,6 & $B_{1} r B_{4}^{\prime} a^{\prime}$ \\
\hline 1928-1947 & 329 & 45 & 1036 & 359 & 32 & 4 & 29 & 34,7 & $B_{1} r B_{4}^{\prime} a^{\prime}$ \\
\hline $1929-1948$ & 334 & 47 & 1034 & 359 & 32 & 5 & 30 & 34,7 & $B_{1} r B_{4}^{\prime} a^{7}$ \\
\hline $1930-1949$ & 328 & 50 & 1032 & 356 & 32 & 5 & 29 & 34,5 & $B_{1} I B_{4}^{\prime} a^{\prime}$ \\
\hline $1931-1950$ & 327 & 50 & 1030 & 357 & 32 & 5 & 29 & 34,7 & $\mathrm{~B}_{1} \mathrm{rB}_{4}^{\prime} \mathrm{a}^{\prime}$ \\
\hline 1932-195] & 314 & 60 & 1029 & 356 & 31 & 6 & 27 & 34,6 & $\mathrm{~B}_{1} \Gamma \mathrm{B}_{4}^{\prime} \mathrm{a}^{\prime}$ \\
\hline $1933-1952$ & 308 & 60 & 1027 & 355 & 30 & 6 & 26 & 34,6 & $\mathrm{~B}_{\mathrm{t}} \mathrm{rB}_{4}^{\prime} \mathrm{a}^{\prime}$ \\
\hline 1934-1953 & 314 & 59 & 1028 & 354 & 31 & 6 & 27 & 34,4 & $\mathrm{~B}_{1} \times \mathrm{B}_{4}^{\prime} \mathrm{a}^{\prime}$ \\
\hline $1935-1954$ & 308 & 54 & 1029 & 355 & 30 & 5 & 27 & 34,5 & $\mathrm{~B}_{1} \times \mathrm{B}_{4}^{\prime} \mathrm{a}^{\prime}$ \\
\hline $1936-1955$ & 309 & 61 & 1028 & 353 & 30 & 6 & 26 & 34,3 & $\mathrm{~B}_{\mathrm{i}} \mathrm{r} \mathrm{B}_{4}^{\prime} \mathrm{a}^{\prime}$ \\
\hline 1937-1956 & 293 & 46 & 1021 & 355 & 29 & 5 & 26 & 34,8 & $\mathrm{~B}_{1} \times \mathrm{B}_{4}^{\prime} \mathrm{a}^{\prime}$ \\
\hline 1938-1957 & 297 & 47 & 1019 & 357 & 29 & 5 & 26 & 35,0 & $\mathrm{~B}_{1} \times \mathrm{B}_{4}^{\prime} \mathrm{a}^{\prime}$ \\
\hline 1939-1958 & 308 & 39 & 1017 & 355 & 30 & 4 & 28 & 34,9 & $\mathrm{~B}_{1} \mathrm{r} \mathrm{B}_{4}^{\prime} \mathrm{a}^{\prime}$ \\
\hline $1940-1959$ & 301 & 46 & 1019 & 357 & 30 & 5 & 27 & 35,0 & $\mathrm{~B}_{1} \mathrm{rB}_{4}^{\prime} \mathrm{a}^{\prime}$ \\
\hline $1941-1960$ & 310 & 39 & 1014 & 353 & 31 & 4 & 28 & 34,8 & $\mathrm{~B}_{1} \times \mathrm{B}_{4}^{\prime} \mathrm{a}^{\prime}$ \\
\hline $1942-1961$ & 298 & 46 & 1015 & 353 & 29 & $s$ & 27 & 34,8 & $B_{1} r B_{4}^{\prime} a^{\prime}$ \\
\hline $1943-1962$ & 314 & 48 & 1013 & 351 & 31 & 5 & 28 & 34,6 & $\mathrm{~B}_{1} \times \mathrm{B}_{4}^{\prime} \mathrm{a}^{\prime}$ \\
\hline $1944-1963$ & 279 & 55 & 1020 & 353 & 27 & 5 & 24 & 34,6 & $\mathrm{~B}_{1} \times \mathrm{BB}_{4}^{\prime} \mathrm{a}^{\prime}$ \\
\hline |1945-1964 & 269 & 49 & 1017 & 352 & 26 & 5 & 24 & 34,6 & $\mathrm{~B}_{1}+\mathrm{B}_{4}^{\prime} \mathrm{a}^{\prime}$ \\
\hline
\end{tabular}


Continuação:

Quadro 3- Classificação climática de Thornthwaite para a ordem de 20 anos (Periodo: 1917-1996)

\begin{tabular}{|c|c|c|c|c|c|c|c|c|c|}
\hline Ano & EXC & DEF & EP & $\mathrm{CV}$ & $\mathrm{Iu}$ & Ia & Im & CVEP & $\mathrm{TH}$ \\
\hline $1946-1965$ & 273 & 53 & 1016 & 351 & 27 & 5 & 24 & 34,5 & $B_{1} \Gamma B_{4}^{\prime} a^{\prime}$ \\
\hline $1947-1966$ & 279 & 51 & 1016 & 353 & 27 & 5 & 24 & 34,7 & $B_{1} \times B_{4}^{\prime} a^{\prime}$ \\
\hline $1948-1967$ & 266 & 53 & 1021 & 351 & 26 & 5 & 23 & 34,4 & $B_{1} \Gamma B_{1}^{\prime} a^{\prime}$ \\
\hline $1949-1968$ & 254 & 53 & 1020 & 351 & 25 & 5 & 22 & 34,4 & $\mathrm{~B}_{1} \times \mathrm{B}_{4}^{\prime} \mathrm{a}^{\prime}$ \\
\hline $1950-1969$ & 236 & 53 & 1024 & 353 & 23 & 5 & 20 & 34,5 & $C_{2} \times B_{4}^{\prime} a^{\prime}$ \\
\hline $1951-1970$ & 222 & 49 & 1024 & 354 & 22 & 5 & 19 & 34,6 & $\mathrm{C}_{2}$ I $B_{4}^{\prime} \mathrm{a}^{\prime}$ \\
\hline $1952-1971$ & 212 & 40 & 1028 & 358 & 21 & 4 & 18 & 34,8 & $\mathrm{C}_{2} \times \mathrm{B}_{4}^{\prime} \mathrm{a}^{\prime}$ \\
\hline $1953-1972$ & 210 & 37 & 1025 & 357 & 20 & 4 & 18 & 34,8 & $\mathrm{C}_{2} \Gamma \mathrm{B}_{4}^{\prime} \mathrm{a}^{\prime}$ \\
\hline 1954-1973 & 203 & 34 & 1026 & 357 & 20 & 3 & 18 & 34,8 & $\mathrm{C}_{2} \mathrm{rB}_{4}^{\prime} \mathrm{a}^{\prime}$ \\
\hline $1955-1974$ & 214 & 35 & 1023 & 357 & 21 & 3 & 19 & 34,9 & $\mathrm{C}_{2} \times B_{4}^{\prime} \mathrm{a}^{\prime}$ \\
\hline $1956-1975$ & 228 & 37 & 1024 & 357 & 22 & 4 & 20 & 34,9 & $\mathrm{C}_{2} \times \mathrm{BB}_{4}^{\prime} \mathrm{a}^{\prime}$ \\
\hline $1957-1976$ & 263 & 40 & 1023 & 355 & 26 & 4 & 23 & 34,7 & $B_{1} \times B_{4}^{\prime} a^{\prime}$ \\
\hline 1958-1977 & 264 & 40 & 1026 & 356 & 26 & 4 & 23 & 34,7 & $B_{1} \simeq B_{4}^{\prime} a^{\prime}$ \\
\hline $1959-1978$ & 241 & 47 & 1025 & 355 & 24 & 5 & 21 & 34,6 & $\mathrm{~B}_{1} \times \mathrm{BB}_{4}^{\prime} \mathrm{a}^{\prime}$ \\
\hline $1960-1979$ & 222 & 38 & 1020 & 353 & 22 & 4 & 20 & 34,6 & $\mathrm{C}_{2} \mathrm{rB}_{4}^{\prime} \mathrm{a}^{\prime}$ \\
\hline $1961-1980$ & 197 & 35 & 1016 & 352 & 19 & 3 & 17 & 34,6 & $\mathrm{C}_{2}$ г $\mathrm{B}_{4}^{\prime} \mathrm{a}^{\prime}$ \\
\hline $1962-1981$ & 213 & 31 & 1013 & 351 & 21 & 3 & 19 & 34,6 & $\mathrm{C}_{2}+B_{4}^{\prime} \mathrm{a}^{\prime}$ \\
\hline $1963-1982$ & 222 & 27 & 1017 & 351 & 22 & 3 & 20 & 34,5 & $\mathrm{C}_{2} \times B_{4}^{\prime} \mathrm{a}^{\prime}$ \\
\hline 1964-1983 & 261 & 6 & 1012 & 352 & 26 & 1 & 25 & 34,8 & $\mathrm{~B}_{1}+\mathrm{B}_{4}^{\prime} \mathrm{a}^{\prime}$ \\
\hline $1965-1984$ & 243 & 7 & 1020 & 353 & 24 & 1 & 23 & 34,6 & $\mathrm{~B}_{1} \times \mathrm{B}_{4}^{\prime} \mathrm{a}^{\prime}$ \\
\hline 1966-1985 & 209 & 6 & 1024 & 356 & 20 & 1 & 20 & 34,8 & $\mathrm{C}_{2} \mathrm{rB}_{4}^{\prime} \mathrm{a}^{\prime}$ \\
\hline $1967-1986$ & 214 & 8 & 1023 & 355 & 21 & 1 & 20 & 34,7 & $\mathrm{C}_{2} \times \mathrm{B}_{4}^{\prime} \mathrm{a}^{\prime}$ \\
\hline 1968-1987 & 223 & 8 & 1027 & 359 & 22 & 1 & 21 & 35,0 & $\mathrm{~B}_{1} \pi \mathrm{B}_{4}^{\prime} \mathrm{a}^{\prime}$ \\
\hline 1969-1988 & 244 & 9 & 1029 & 360 & 24 & 1 & 23 & 35,0 & $\mathrm{~B}_{1} \times \mathrm{B}_{4}^{\prime} \mathrm{a}^{\prime}$ \\
\hline $1970-1989$ & 260 & 8 & 1028 & 360 & 25 & 1 & 25 & 35,0 & $\mathrm{~B}_{1} \mathrm{rB}_{4}^{\prime} \mathrm{a}^{\prime}$ \\
\hline $1971-1990$ & 254 & 6 & 1032 & 362 & 25 & 1 & 24 & 35,1 & $\mathrm{~B}_{1} \times \mathrm{B}_{4}^{\prime} \mathrm{a}^{\prime}$ \\
\hline $1972-1991$ & 274 & 6 & 1030 & 359 & 27 & 1 & 26 & 34,9 & $B_{1} \mp B_{4}^{\prime} a^{\prime}$ \\
\hline 1973-1992 & 277 & 7 & 1036 & 361 & 27 & 1 & 26 & 34,8 & $\mathrm{~B}_{1} \times \mathrm{B}_{4}^{\prime} \mathrm{a}^{\prime}$ \\
\hline $1974-1993$ & 290 & 6 & 1039 & 361 & 28 & 1 & 28 & 34,7 & $\mathrm{~B}_{1}$ I $B_{4}^{\prime} \mathrm{a}^{\prime}$ \\
\hline $1975-1994$ & 290 & 6 & 1047 & 363 & 28 & 1 & 27 & 34,7 & $\mathrm{~B}_{1} \times \mathrm{B}_{4}^{\prime} \mathrm{a}^{\prime}$ \\
\hline $1976-1995$ & 304 & 5 & 1050 & 365 & 29 & 0 & 29 & 34,8 & $\mathrm{~B}_{1} \times \mathrm{B}_{4}^{\prime} \mathrm{a}^{\prime}$ \\
\hline $1977-1996$ & 293 & 8 & 1057 & 367 & 28 & 1 & 27 & 34,7 & $\mathrm{~B}_{1} \times \mathrm{B}_{4}^{\prime} \mathrm{a}^{\prime}$ \\
\hline
\end{tabular}

Quadro 4- Classificação climática de Thornthwaite para a ordem de 25 anos (Período: 1917-1996)

\begin{tabular}{|c|c|c|c|c|c|c|c|c|c|}
\hline Ano & EXC & DEF & EP & CV & Iu & Ia & Im & CVEP & TH \\
\hline $1917-1941$ & 242 & 29 & 1027 & 257 & 24 & 3 & 22 & 25,0 & $B_{1} \mathrm{BB}_{4}^{\prime} \mathrm{a}^{\prime}$ \\
\hline $1918-1942$ & 243 & 30 & 1032 & 357 & 24 & 3 & 22 & 34,6 & $\mathrm{~B}_{1} \mathrm{rB}_{4}^{\prime} \mathrm{a}^{\prime}$ \\
\hline $1919-1943$ & 264 & 30 & 1032 & 358 & 26 & 3 & 24 & 34,7 & $\mathrm{~B}_{1} \mathrm{rB}_{4}^{\prime} \mathrm{a}^{\prime}$ \\
\hline $1920-1944$ & 274 & 37 & 1032 & 358 & 27 & 4 & 24 & 34,7 & $\mathrm{~B}_{1} \mathrm{rB}_{4}^{\prime} \mathrm{a}^{\prime}$ \\
\hline $1921-1945$ & 281 & 32 & 1034 & 358 & 27 & 3 & 25 & 34,6 & $\mathrm{~B}_{1} \mathrm{rB}_{4}^{\prime} \mathrm{a}^{\prime}$ \\
\hline $1922-1946$ & 287 & 32 & 1035 & 257 & 28 & 3 & 26 & 24,8 & $\mathrm{~B}_{1} \mathrm{rB}_{4}^{\prime} \mathrm{a}^{\prime}$ \\
\hline $1923-1947$ & 302 & 33 & 1034 & 359 & 29 & 3 & 27 & 34,7 & $\mathrm{~B}_{1} \mathrm{rB}_{4}^{\prime} \mathrm{a}^{\prime}$ \\
\hline $1924-1948$ & 301 & 39 & 1033 & 358 & 29 & 4 & 27 & 34,7 & $\mathrm{~B}_{1} \mathrm{rB}_{4}^{\prime} \mathrm{a}^{\prime}$ \\
\hline $1925-1949$ & 317 & 37 & 1033 & 358 & 31 & 4 & 29 & 34,7 & $\mathrm{~B}_{1} \mathrm{rB}_{4}^{\prime} \mathrm{a}^{\prime}$ \\
\hline $1926-1950$ & 337 & 38 & 1031 & 356 & 33 & 4 & 30 & 34,5 & $\mathrm{~B}_{1} \mathrm{BB}_{4}^{\prime} \mathrm{a}^{\prime}$ \\
\hline $1927-1951$ & 336 & 50 & 1029 & 356 & 33 & 5 & 30 & 34,6 & $\mathrm{~B}_{1} \mathrm{rB}_{4}^{\prime} \mathrm{a}^{\prime}$ \\
\hline $1928-1952$ & 334 & 55 & 1028 & 255 & 32 & 5 & 29 & 24,8 & $\mathrm{~B}_{1} \mathrm{rB}_{4}^{\prime} \mathrm{a}^{\prime}$
\end{tabular}


Continuação:

Quadro 4- Classificação climática de Thornthwaite para a ordem de 25 anos (Periodo: 1917-1996)

\begin{tabular}{|c|c|c|c|c|c|c|c|c|c|}
\hline Ano & EXC & DEF & EP & $\mathrm{CV}$ & $\mathbf{I u}$ & Ia & Im & CVEP & $\mathrm{TH}$ \\
\hline $1929-1953$ & 331 & 53 & 1026 & 355 & 32 & 5 & 29 & 34,6 & $\mathrm{~B}_{1} \mathrm{BB}_{4}^{\prime} \mathrm{a}^{\prime}$ \\
\hline 1930-1954 & 312 & 55 & 1028 & 355 & 30 & 5 & 27 & 34,5 & $\mathrm{~B}_{1} \mathrm{IB}_{4}{ }_{4} \mathrm{a}^{\prime}$ \\
\hline 1931-1955 & 299 & 54 & 1028 & 357 & 29 & 5 & 26 & 34,7 & $\mathrm{~B}_{1} \mathrm{IB}_{4}^{\prime} \mathrm{a}^{\prime}$ \\
\hline $1932-1956$ & 269 & 50 & 1028 & 357 & 26 & 5 & 23 & 34,7 & $\mathrm{~B}_{1} \mathrm{rB}_{4}^{\prime} \mathrm{a}^{\prime}$ \\
\hline $1933-1957$ & 267 & 46 & 1025 & 356 & 26 & 4 & 23 & 34,7 & $\mathrm{~B}_{\mathrm{H}} \mathrm{rB}_{4}^{\prime} \mathrm{a}^{\prime}$ \\
\hline 1934-1958 & 279 & 37 & 1026 & 257 & 27 & 4 & 25 & 25,0 & $\mathrm{~B}_{1} \mathrm{rB}_{4}^{\prime} \mathrm{a}^{\prime}$ \\
\hline $1935-1959$ & 285 & 37 & 1028 & 358 & 28 & 4 & 26 & 34,8 & $\mathrm{~B}_{1} \mathrm{rB}_{4}^{\prime} \mathrm{a}^{\prime}$ \\
\hline $1936-1960$ & 302 & 42 & 1025 & 356 & 29 & 4 & 27 & 34,7 & $\mathrm{~B}_{\mathrm{I}} \mathrm{BB}_{4}^{\prime} \mathrm{a}^{\prime}$ \\
\hline $1937-1961$ & 296 & 44 & 1023 & 355 & 29 & 4 & 26 & 34,7 & $\mathrm{~B}_{1} \mathrm{IB}_{4}^{\prime} \mathrm{a}^{\prime}$ \\
\hline $1938-1962$ & 309 & 49 & 1021 & 355 & 30 & 5 & 27 & 34,8 & $\mathrm{~B}_{1} \mathrm{rB}_{4}{ }_{4} \mathrm{a}^{\prime}$ \\
\hline $1939-1963$ & 305 & 57 & 1023 & 355 & 30 & 6 & 26 & 34,7 & $\mathrm{~B}_{1} \mathrm{rB}_{4}^{\prime} \mathrm{a}^{\prime}$ \\
\hline $1940-1964$ & 290 & 60 & 1021 & 352 & 28 & 6 & 25 & 34,5 & $\mathrm{~B}_{1} \mathrm{~TB}_{4}^{\prime} \mathrm{a}^{\prime}$ \\
\hline 1941-1965 & 295 & 54 & 1017 & 352 & 29 & 5 & 26 & 34,6 & $\mathrm{~B}_{1} \mathrm{BB}_{4}^{\prime} \mathrm{a}^{\prime}$ \\
\hline $1942-1966$ & 294 & 57 & 1017 & 352 & 29 & 6 & 26 & 34,6 & $\mathrm{~B}_{1} \mathrm{IB}_{4}^{\prime} \mathrm{a}^{\prime}$ \\
\hline $1943-1967$ & 298 & 60 & 1020 & 351 & 29 & 6 & 26 & 34,4 & $\mathrm{~B}_{\mathrm{f}} \mathrm{rB}_{4}^{\prime} \mathrm{a}^{\prime}$ \\
\hline 19441968 & 273 & 60 & 1019 & 352 & 27 & 6 & 23 & 34,5 & $\mathrm{~B}_{1} \mathrm{rB}_{4}^{\prime} \mathrm{a}^{\prime}$ \\
\hline $1945-1969$ & 265 & 55 & 1019 & 352 & 26 & 5 & 23 & 34,5 & $\mathrm{~B}_{1} \mathrm{rB}_{4}^{\prime} \mathrm{a}^{\prime}$ \\
\hline $1946-1970$ & 256 & 54 & 1019 & 353 & 25 & 5 & 22 & 34,6 & $\mathrm{~B}_{1} \mathrm{rB}_{4}^{\prime} \mathrm{a}^{\prime}$ \\
\hline $1947-1971$ & 257 & 50 & 1021 & 354 & 25 & 5 & 22 & 34,7 & $\mathrm{~B}_{1} \mathrm{BB}_{4}{ }_{4} \mathrm{a}^{\prime}$ \\
\hline $1948-1972$ & 241 & 46 & 1019 & 353 & 24 & 5 & 21 & 34,6 & $\mathrm{~B}_{2} \mathrm{rB}_{4}^{\prime} \mathrm{a}^{\prime}$ \\
\hline $1949-1973$ & 227 & 42 & 1020 & 354 & 22 & 4 & 20 & 34,7 & $\mathrm{C}_{2} \mathrm{rB}_{4}^{\prime} \mathrm{a}^{\prime}$ \\
\hline $1950-1974$ & 222 & 42 & 1020 & 355 & 22 & 4 & 19 & 34,8 & $\mathrm{C}_{2} \times \mathrm{B}_{4}^{\prime} \mathrm{a}^{\prime}$ \\
\hline $1951-1975$ & 218 & 41 & 1023 & 356 & 21 & 4 & 19 & 34,8 & $\mathrm{C}_{2} \times \mathrm{B}_{4}^{\prime} \mathrm{a}^{\prime}$ \\
\hline $1952-1976$ & 228 & 33 & 1021 & 356 & 22 & 3 & 20 & 34,9 & $\mathrm{C}_{2} \times \mathrm{BB}_{4}^{\prime} \mathrm{a}^{\prime}$ \\
\hline 1953-1977 & 230 & 32 & 1025 & 357 & 22 & 3 & 21 & 34,8 & $\mathrm{~B}_{1} \mathrm{rB}_{4}^{\prime} \mathrm{a}^{\prime}$ \\
\hline $1954-1978$ & 221 & 31 & 1024 & 357 & 22 & 3 & 20 & 34,9 & $\mathrm{C}_{2} \mathrm{IB}_{4}^{\prime} \mathrm{a}^{\prime}$ \\
\hline $1955-1979$ & 219 & 26 & 1019 & 355 & 21 & 3 & 20 & 34,8 & $\mathrm{C}_{2} \times \mathrm{B}_{4}^{\prime} \mathrm{a}^{\prime}$ \\
\hline $1956-1980$ & 213 & 26 & 1017 & 353 & 21 & 3 & 19 & 34,7 & $\mathrm{C}_{2} \times \mathrm{B}_{4}^{\prime} \mathrm{a}^{\prime}$ \\
\hline 1957.1981 & 230 & 33 & 1018 & 353 & 23 & 3 & 21 & 34,7 & $\mathrm{~B}_{1} \mathrm{rB}_{4}^{\prime} \mathrm{a}^{\prime}$ \\
\hline $1958-1982$ & 247 & 33 & 1019 & 350 & 24 & 3 & 22 & 34,3 & $\mathrm{~B}_{1} \mathrm{rB}_{4}{ }_{4} \mathrm{a}^{\prime}$ \\
\hline $1959-1983$ & 257 & 23 & 1019 & 351 & 25 & 2 & 24 & 34,4 & $\mathrm{~B}_{1} \mathrm{rB}_{4}^{\prime} \mathrm{a}^{\prime}$ \\
\hline $1960-1984$ & 233 & 20 & 1022 & 353 & 23 & 2 & 22 & 34,5 & $\mathrm{~B}_{1} \mathrm{rB}_{4}^{\prime} \mathrm{a}^{\prime}$ \\
\hline $1961-1985$ & 208 & 18 & 1023 & 353 & 20 & 2 & 19 & 34,5 & $\mathrm{C}_{2} \mathrm{rB}_{4}^{\prime} \mathrm{a}^{\prime}$ \\
\hline $1962-1986$ & 218 & 15 & 1025 & 355 & 21 & 1 & 20 & 34,6 & $\mathrm{C}_{2} \mathrm{rB_{4 } ^ { \prime }} \mathrm{a}^{\prime}$ \\
\hline 1963-1987 & 215 & 11 & 1028 & 357 & 21 & 1 & 20 & 34,7 & $\mathrm{C}_{2}+\mathrm{BB}_{4}^{\prime} \mathrm{a}^{\prime}$ \\
\hline $1964-1988$ & 235 & 10 & 1027 & 358 & 23 & 1 & 22 & 34,9 & $\mathrm{~B}_{1} \mathrm{rB}_{4}^{\prime} \mathrm{a}^{\prime}$ \\
\hline 1965-1989 & 251 & 9 & 1029 & 359 & 24 & 1 & 24 & 34,9 & $\mathrm{~B}_{1} \mathrm{IB}_{4}^{\prime} \mathrm{a}^{\prime}$ \\
\hline $1966-1990$ & 235 & 7 & 1033 & 361 & 23 & 1 & 22 & 34,9 & $\mathrm{~B}_{4} \mathrm{rB}_{4}^{\prime} \mathrm{a}^{\prime}$ \\
\hline $1967-1991$ & 252 & 7 & 1031 & 360 & 24 & 1 & 24 & 34,9 & $\mathrm{~B}_{1} \mathrm{rB}_{4}^{\prime} \mathrm{a}^{\prime}$ \\
\hline $1968-1992$ & 252 & 6 & 1032 & 361 & 24 & 1 & 24 & 35,0 & $\mathrm{~B}_{1} \mathrm{rB}_{4}^{\prime} \mathrm{a}^{\prime}$ \\
\hline $1969-1993$ & 258 & 5 & 1037 & 362 & 25 & 0 & 25 & 34,9 & $\mathrm{~B}_{1} \mathrm{rB}_{4}^{\prime} \mathrm{a}^{\prime}$ \\
\hline $1970-1994$ & 269 & 6 & 1040 & 363 & 26 & 1 & 26 & 34,9 & $\mathrm{~B}_{1} \mathrm{rB}_{4}^{\prime} \mathrm{a}^{\prime}$ \\
\hline 1971-1995 & 279 & 8 & 1044 & 364 & 27 & 1 & 26 & 34,9 & $\mathrm{~B}_{1} \mathrm{rB}_{4}^{\prime} \mathrm{a}^{\prime}$ \\
\hline $1972-1996$ & 290 & 8 & 1043 & 364 & 28 & 1 & 27 & 34,9 & $\mathrm{~B}_{1} \mathrm{IB}_{4}^{\prime} \mathrm{a}^{\prime}$ \\
\hline
\end{tabular}


Quadro 5- Classificação climática de Thornthwaite para a ordem de 30 anos (Período: 1917-1996)

\begin{tabular}{|c|c|c|c|c|c|c|c|c|c|}
\hline Ano & EXC & DEF & EP & $\mathrm{CV}$ & Iu & Ia & Im & CVEP & $\mathrm{TH}$ \\
\hline $1917-1946$ & 256 & 30 & 1026 & 337 & 24,95 & 2,92 & 23 & 32,85 & $\mathrm{~B}_{1} \times \mathrm{B}_{4}^{\prime} \mathrm{a}^{\prime}$ \\
\hline $1918-1947$ & 273 & 32 & 1027 & 354 & 26,58 & 3,12 & 25 & 34,47 & $B_{1} \times B_{4}^{\prime} a^{\prime}$ \\
\hline $1919-1948$ & 275 & 33 & 1030 & 357 & 26,70 & 3,20 & 25 & 34,66 & $\mathrm{~B}_{1} \times \mathrm{B}_{4}^{\prime} \mathrm{a}^{\prime}$ \\
\hline $1920-1949$ & 288 & 37 & 1029 & 357 & 27,99 & 3,60 & 26 & 34,69 & $\mathrm{~B}_{1} \mathbf{r} \mathbf{B}_{4}^{\prime} \mathbf{a}^{\prime}$ \\
\hline $1921-1950$ & 297 & 38 & 1028 & 357 & 28,89 & 3,70 & 27 & 34,73 & $\mathrm{~B}_{1}$ r $\mathrm{B}_{\mathbf{4}}^{\prime} \mathrm{a}^{\prime}$ \\
\hline $1922-1951$ & 314 & 40 & 1026 & 354 & 30,60 & 3,90 & 28 & 34,50 & $\mathrm{~B}_{1}+\mathrm{B}_{4}^{\prime} \mathrm{a}^{\prime}$ \\
\hline 1923-1952 & 306 & 39 & 1029 & 355 & 29,74 & 3,79 & 27 & 34,50 & $B_{1} \times B_{4}^{\prime} a^{\prime}$ \\
\hline 1924-1953 & 304 & 47 & 1027 & 355 & 29,60 & 4,58 & 27 & 34,57 & $B_{1} r B_{4}^{\prime} a^{\prime}$ \\
\hline $1925-1954$ & 307 & 44 & 1029 & 354 & 29,83 & 4,28 & 27 & 34,40 & $\mathbf{B}_{1} \times \mathbf{B}_{4}^{\prime} \mathbf{a}^{\prime}$ \\
\hline $1926-1955$ & 313 & 42 & 1028 & 355 & 30,45 & 4,09 & 28 & 34,53 & $B_{1} r B_{4}^{\prime} a^{\prime}$ \\
\hline $1927-1956$ & 294 & 44 & 1027 & 357 & 28,63 & 4,28 & 26 & 34,76 & $\mathrm{~B}_{1} \times \mathrm{B}_{4}^{\prime} \mathrm{a}^{\prime}$ \\
\hline $1928-1957$ & 295 & 43 & 1025 & 356 & 28,78 & 4,20 . & 26 & 34,73 & $\mathrm{~B}_{1} \times \mathrm{B}_{4}^{\prime} \mathrm{a}^{\prime}$ \\
\hline $1929-1958$ & 299 & 37 & 1025 & 357 & 29,17 & 3,61 & 27 & 34,83 & $B_{1} r B_{4}^{\prime} a^{\prime}$ \\
\hline $1930-1959$ & 293 & 41 & 1027 & 357 & 28,53 & 3,99 & 26 & 34,76 & $\mathrm{~B}_{1} \times \mathrm{B}_{4}^{\prime} \mathrm{a}^{\prime}$ \\
\hline $1931-1960$ & 295 & 40 & 1027 & 357 & 28,72 & 3,89 & 26 & 34,76 & $\mathrm{~B}_{1} \times \mathrm{B}_{4}^{\prime} \mathrm{a}^{\prime}$ \\
\hline 1932-1961 & 274 & 45 & 1029 & 356 & 26,63 & 4,37 & 24 & 34,60 & $B_{1} \times B_{4}^{\prime} a^{\prime}$ \\
\hline $1933-1962$ & 282 & 45 & 1026 & 356 & 27,49 & 4,39 & 25 & 34,70 & $\mathrm{~B}_{1} \mathrm{r} \mathrm{B}_{4}^{\prime} \mathrm{a}^{\prime}$ \\
\hline $1934-1963$ & 283 & 50 & 1030 & 356 & 27,48 & 4,85 & 25 & 34,56 & $\mathrm{~B}_{1}$ 工 $\mathrm{B}_{4}^{\prime} \mathrm{a}^{\prime}$ \\
\hline $1935-1964$ & 280 & 50 & 1026 & 354 & 27,29 & 4,87 & 24 & 34,50 & $\mathrm{~B}_{1} \times \mathrm{B}_{4}^{\prime} \mathbf{a}^{\prime}$ \\
\hline 1936-1965 & 289 & 51 & 1028 & 354 & 28,11 & 4,96 & 25 & 34,44 & $\mathrm{~B}_{1} \mathbf{r} \mathbf{B}_{4}^{\prime} \mathrm{a}^{\mathbf{2}}$ \\
\hline 1937-1966 & 293 & 52 & 1028 & 355 & 28,50 & 5,06 & 25 & 34,53 & $\mathrm{~B}_{1} \times \mathrm{B}_{4}^{\prime} \mathrm{a}^{\prime}$ \\
\hline $1938-1967$ & 297 & 57 & 1025 & 354 & 28,98 & 5,56 & 26 & 34,54 & $\mathbf{B}_{\mathbf{1}} \mathbf{r} \mathbf{B}_{\mathbf{4}}^{\prime} \mathbf{a}^{\mathbf{\prime}}$ \\
\hline $1939-1968$ & 297 & 59 & 1022 & 352 & 29,06 & 5,77 & 26 & 34,44 & $B_{1} r B_{4}^{\prime} a^{\prime}$ \\
\hline $1940-1969$ & 284 & 62 & 1022 & 354 & 27,79 & 6,07 & 24 & 34,64 & $\mathrm{~B}_{1} \times \mathrm{B}_{4}^{\prime} \mathrm{a}^{\mathbf{\prime}}$ \\
\hline $1941-1970$ & 278 & 53 & 1020 & 353 & 27,25 & 5,20 & 24 & 34,61 & $B_{1} \times B_{4}^{\prime} \mathbf{a}^{\prime}$ \\
\hline $1942-1971$ & 275 & 54 & 1021 & 354 & 26,93 & 5,29 & 24 & 34,67 & $B_{1} r B_{4}^{\prime} a^{\prime}$ \\
\hline 1943-1972 & 273 & 50 & 1018 & 353 & 26,82 & 4,91 & 24 & 34,68 & $B_{1} \times B_{4}^{\prime} a^{\prime}$ \\
\hline $1944-1973$ & 249 & 47 & 1020 & 354 & 24,41 & 4,61 & 22 & 34,71 & $\mathrm{~B}_{1} \times \mathrm{B}_{4}^{\prime} \mathrm{a}^{+}$ \\
\hline $1945-1974$ & 250 & 42 & 1018 & 354 & 24,56 & 4,13 & 22 & 34,77 & $\mathrm{~B}_{1}+\mathrm{B}_{4}{ }_{4} \mathrm{a}^{\prime}$ \\
\hline 1946-1975 & 246 & 49 & 1019 & 355 & 24,14 & 4,81 & 21 & 34,84 & $B_{1} \times B_{4}^{\prime} a^{\prime}$ \\
\hline $1947-1976$ & 262 & 42 & 1016 & 354 & 25,79 & 4,13 & 23 & 34,84 & $\mathrm{~B}_{1} \times \mathrm{B}_{4}^{\prime} \mathrm{a}^{\prime}$ \\
\hline $1948-1977$ & 251 & 42 & 1020 & 354 & 24,61 & 4,12 & 22 & 34,71 & $B_{1} \times B_{4}^{\prime} a^{\prime}$ \\
\hline 1949-1978 & 236 & 40 & 1019 & 354 & 23,16 & 3,93 & 21 & 34,74 & $\mathrm{~B}_{1} \times \mathrm{B}_{4}^{\prime} \mathrm{a}^{\prime}$ \\
\hline $1950-1979$ & 220 & 34 & 1019 & 355 & 21,59 & 3,34 & 20 & 34,84 & $\mathrm{C}_{2} \times \mathrm{B}_{4}^{\prime} \mathrm{a}^{\prime}$ \\
\hline $1951-1980$ & 210 & 30 & 1016 & 353 & 20,67 & 2,95 & 19 & 34,74 & $\mathrm{C}_{2} \times \mathrm{B}_{4}^{\prime} \mathrm{a}^{\prime}$ \\
\hline 1952-1981 & 206 & 30 & 1018 & 354 & 20,24 & 2,95 & 18 & 34,77 & $\mathrm{C}_{2} \times \mathrm{B}_{4}^{\prime} \mathrm{a}^{\prime}$ \\
\hline $1953-1982$ & 222 & 26 & 1019 & 354 & 21,79 & 2,55 & 20 & 34,74 & $\mathrm{C}_{2} \mathrm{rB}_{4}^{\prime} \mathrm{a}^{\prime}$ \\
\hline 1954-1983 & 241 & 16 & 1020 & 355 & 23,63 & 1,57 & 23 & 34,80 & $\mathrm{~B}_{1} \times \mathrm{B}_{4}^{\prime} \mathrm{a}^{\prime}$ \\
\hline $1955-1984$ & 233 & 14 & 1020 & 355 & 22,84 & 1,37 & 22 & 34,80 & $B_{1} r B_{4}^{\prime} a^{\prime}$ \\
\hline $1956-1985$ & 221 & 13 & 1023 & 355 & 21,60 & 1,27 & 21 & 34,70 & $\mathrm{~B}_{1} \times \mathrm{B}_{4}^{\prime} \mathrm{a}^{\prime}$ \\
\hline $1957-1986$ & 233 & 17 & 1028 & 356 & 22,67 & 1,65 & 22 & 34,63 & $\mathrm{~B}_{1} \times \mathrm{B}_{4}^{\prime} \mathrm{a}^{\prime}$ \\
\hline 1958-1987 & 237 & 15 & 1030 & 357 & 23,01 & 1,46 & 22 & 34,66 & $B_{1} \times B_{4}^{\prime} a^{\prime}$ \\
\hline $1959-1988$ & 238 & 19 & 1031 & 357 & 23,08 & 1,84 & 22 & 34,63 & $\mathrm{~B}_{1} \times \mathrm{B}_{4}^{\prime} \mathrm{a}^{4}$ \\
\hline $1960-1989$ & 241 & 16 & 1029 & 357 & 23,42 & 1.55 & 22 & 34.69 & $\mathrm{~B}_{1} \times \mathrm{B}_{4}^{\prime} \mathrm{a}^{\prime}$ \\
\hline $1961-1990$ & 228 & 14 & 1032 & 358 & 22,09 & 1,36 & 21 & 34,69 & $\mathrm{~B}_{1} \mathrm{rB}_{4}^{\prime} \mathrm{a}^{\prime}$ \\
\hline
\end{tabular}


Continuação:

Quadro 5- Classificação climática de Thornthwaite para a ordem de 30 anos (Período: 1917-1996)

\begin{tabular}{|c|c|c|c|c|c|c|c|c|c|} 
Ano & EXC & DEF & EP & CV & lu & la & Im & CVEP & TH \\
\hline $1962-1991$ & 245 & 12 & 1031 & 359 & 23,76 & 1,16 & 23 & 34,82 & $B_{1} r \mathrm{~B}_{4}^{\prime} \mathrm{a}^{\prime}$ \\
\hline $1963-1992$ & 238 & 11 & 1035 & 360 & 23,00 & 1,06 & 22 & 34,78 & B $_{1} \times \mathrm{B}_{4}^{\prime} \mathrm{a}^{\prime}$ \\
\hline $1964-1993$ & 246 & 7 & 1034 & 360 & 23,79 & 0,68 & 23 & 34,82 & $\mathrm{~B}_{1} \times \mathrm{B}_{4}^{\prime} \mathrm{a}^{\prime}$ \\
\hline $1965-1994$ & 259 & 8 & 1037 & 361 & 24,98 & 0,77 & 25 & 34,81 & $\mathrm{~B}_{1} \times \mathrm{B}_{4}^{\prime} \mathrm{a}^{\prime}$ \\
\hline $1966-1995$ & 259 & 9 & 1041 & 363 & 24,88 & 0,86 & 24 & 34,87 & $\mathrm{~B}_{1} \mathrm{rB}_{4}^{\prime} \mathrm{a}^{\prime}$ \\
\hline $1967-1996$ & 270 & 8 & 1041 & 363 & 25,94 & 0,77 & 25 & 34,87 & $\mathrm{~B}_{1} \times \mathrm{B}_{4}^{\prime} \mathrm{a}^{\prime}$ \\
\hline
\end{tabular}

Quadro 6- Classificação climática de Thornthwaite para a ordent de 35 anos (Período: 1917-1996)

\begin{tabular}{|c|c|c|c|c|c|c|c|c|c|}
\hline Ano & EXC & DEF & EP & $\mathrm{CV}$ & Iu & Ia & Im & CVEP & TH \\
\hline 1917-1951 & 280 & 38 & 1021 & 353 & 27,4 & 3,7 & 25 & 34,6 & $\mathrm{~B} 1 \mathrm{rB}^{\prime} 4 \mathrm{a}^{\prime}$ \\
\hline $1918-1952$ & 283 & 40 & 1023 & 355 & 27,7 & 3,9 & 25 & 34,7 & B1 rB'4 $a^{\prime}$ \\
\hline $1919-1953$ & 282 & 39 & 1024 & 355 & 27,5 & 3,8 & 25 & 34,7 & 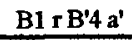 \\
\hline $1920-1954$ & 282 & 43 & 1026 & 356 & 27,5 & 4,2 & 25 & 34,7 & $\mathrm{~B} \mathrm{rB}^{\prime} 4 \mathrm{a}^{\prime}$ \\
\hline $1921-1955$ & 281 & 43 & 1027 & 356 & 27,4 & 4,2 & 25 & 34,7 & B1 rB'4 $\mathrm{a}^{\prime}$ \\
\hline $1922-1956$ & 280 & 36 & 1026 & 356 & 27,3 & 3,5 & 25 & 34,7 & $\mathrm{~B}_{1} \mathrm{rB} \mathrm{B}^{\prime} \mathrm{a}^{\prime}$ \\
\hline $1923-1957$ & 279 & 32 & 1025 & 356 & 27,2 & 3,1 & 25 & 34,7 & B1 $\mathrm{BB}^{\prime} 4 \mathrm{a}^{\prime}$ \\
\hline $1924-1958$ & 281 & 33 & 1025 & 356 & 27,4 & 3,2 & 25 & 34,7 & $\mathrm{~B}^{\prime} \times \mathrm{B}^{\prime} 4 \mathrm{a}^{\prime}$ \\
\hline $1925-1959$ & 291 & 32 & 1027 & 358 & 28,3 & 3,1 & 26 & 34,9 & B1 r B' $4 \mathrm{a}^{\prime}$ \\
\hline $1926-1960$ & 307 & 33 & 1027 & 357 & 29,9 & 3,2 & 28 & 34,8 & $B 1 \times B^{\prime} 4 a^{\prime}$ \\
\hline $1927-1961$ & 296 & 41 & 1029 & 356 & 28,8 & 4,0 & 26 & 34,6 & $\mathrm{~B}^{\prime}+\mathrm{B}^{\prime} 4 \mathrm{a}^{\circ}$ \\
\hline $1928-1962$ & 302 & 44 & 1027 & 357 & 29,4 & 4,3 & 27 & 34,8 & $B_{1}$ r B $4 a^{\prime}$ \\
\hline $1929-1963$ & 316 & 47 & 1030 & 356 & 30,7 & 4,6 & 28 & 34,6 & $\mathrm{~B} 1 \mathrm{rB}^{\prime} 4 \mathrm{a}^{\prime}$ \\
\hline 1930-1964 & 288 & 49 & 1027 & 354 & 28,0 & 4,8 & 25 & 34,5 & B1 r B'4 $a^{\prime}$ \\
\hline $1931-1965$ & 287 & 49 & 1026 & 354 & 28,0 & 4,8 & 25 & 34,5 & B1 r B $4 a^{\prime}$ \\
\hline $1932-1966$ & 276 & 52 & 1028 & 356 & 26,8 & 5,1 & 24 & 34,6 & $\mathrm{~B}^{1} \mathrm{rB}^{\prime} 4 \mathrm{a}^{\prime}$ \\
\hline 1933-1967 & 276 & 52 & 1029 & 356 & 26,8 & 5,1 & 24 & 34,6 & $\mathrm{~B} 1 \mathrm{rB}^{4} 4 \mathrm{a}^{\prime}$ \\
\hline 1934-1968 & 277 & 54 & 1028 & 355 & 26,9 & 5,3 & 24 & 34,5 & $\mathrm{~B} 1 \mathrm{r} \mathrm{B}^{\prime} 4 \mathrm{a}^{\prime}$ \\
\hline $1935-1969$ & 275 & 52 & 1027 & 354 & 26,8 & 5,1 & 24 & 34,5 & B1 rB $4 \mathrm{a}^{\prime}$ \\
\hline $1936-1970$ & 278 & 53 & 1027 & 354 & 27,1 & 5,2 & 24 & 34,5 & $\mathrm{~B}^{\prime}$ r B'4 $\mathrm{a}^{\prime}$ \\
\hline $1937-1971$ & 277 & so & 1025 & 356 & 27,0 & 4,9 & 24 & 34,7 & $\mathrm{Bl} \times \mathrm{B}^{\prime} 4 \mathrm{a}^{\prime}$ \\
\hline 1938-1972 & 275 & 48 & 1023 & 356 & 26,9 & 4,7 & 24 & 34,8 & $\mathrm{~B} 1+\mathrm{B}^{\prime} 4 \mathrm{a}^{\mathrm{a}}$ \\
\hline 1939-1973 & 272 & 49 & 1021 & 355 & 26,6 & 4,8 & 24 & 34,8 & $\mathrm{~B} 1 \mathrm{rB}^{\prime} 4 \mathrm{a}^{*}$ \\
\hline $1940-1974$ & 266 & 51 & 1022 & 356 & 26,0 & 5,0 & 23 & 34,8 & $\mathrm{Bl} \times \mathrm{B}^{\prime} 4 \mathrm{a}^{\prime}$ \\
\hline $1941-1975$ & 267 & 48 & 1020 & 355 & 26,2 & 4,7 & 23 & 34,8 & $\mathrm{~B}^{\prime} r \mathrm{~B}^{\prime} 4 \mathrm{a}^{\prime}$ \\
\hline $1942-1976$ & 277 & 45 & 1017 & 353 & 27,2 & 4,4 & 25 & 34,7 & $\mathrm{~B}^{\prime} \mathrm{rB} \mathrm{B}^{\prime} \mathrm{a}^{\prime}$ \\
\hline $1943-1977$ & 276 & 45 & 1019 & 354 & 27,1 & 4,4 & 24 & 34,7 & $\mathrm{~B} 1 \mathrm{rB}^{\prime} 4 \mathrm{a}^{\prime}$ \\
\hline $1944-1978$ & 253 & 43 & 1019 & 355 & 24,8 & 4,2 & 22 & 34,8 & $\mathrm{~B} 1 \mathrm{r} \mathrm{B}^{\prime} 4 \mathrm{a}^{\prime}$ \\
\hline 1945-1979 & 244 & 36 & 1016 & 354 & 24,0 & 3,5 & 22 & 34,8 & B1 r $\mathrm{B}^{\prime} 4 \mathrm{a}^{\prime}$ \\
\hline $1946-1980$ & 233 & 38 & 1014 & 352 & 23,0 & 3,7 & 21 & 34,7 & $\mathrm{~B}^{\prime}$ r B'4 $\mathrm{a}^{\prime}$ \\
\hline $1947-1981$ & 239 & 37 & 1014 & 352 & 23,6 & 3,6 & 21 & 34,7 & B1 r B'4 a' \\
\hline $1948-1982$ & 242 & 35 & 1014 & 351 & 23,9 & 3,5 & 22 & 34,6 & $\mathrm{~B} 1 \mathrm{rB}^{\prime} 4 \mathrm{a}^{\prime}$ \\
\hline $1949-1983$ & 251 & 23 & 1015 & 351 & 24,7 & 2,3 & 23 & 34,6 & BI r B'4 $a^{\prime}$ \\
\hline $1950-1984$ & 232 & 21 & 1020 & 354 & 22,7 & 2,1 & 22 & 34,7 & $\mathrm{Bl}^{\prime} \mathrm{rB}^{\prime} 4 \mathrm{a}^{\prime}$ \\
\hline 1951-1985 & 216 & 19 & 1021 & 355 & 21,2 & 1,9 & 20 & 34,8 & $\mathrm{C} 2 \mathrm{IB}^{\prime} 4 \mathrm{a}^{\prime}$ \\
\hline $1952-1986$ & 213 & 16 & 1025 & 356 & 20,8 & 1,6 & 20 & 34,7 & $\mathrm{C} 2 \mathrm{r} \mathrm{B}^{\prime} 4 \mathrm{a}^{\prime}$ \\
\hline |1953-1987 & 218 & 14 & 1028 & 358 & 21,2 & 1,4 & 20 & 34,8 & $\mathrm{C} 2 \mathrm{r} \mathrm{B}^{\prime} 4 \mathrm{a}^{\prime}$ \\
\hline
\end{tabular}


Continuação:

Quadro 6- Classificação climática de Thornthwaite para a ordem de 35 anos (Periodo: 1917-1996)

\begin{tabular}{|c|c|c|c|c|c|c|c|c|c|}
\hline Ano & EXC & DEF & EP & $\mathrm{CV}$ & $\mathbf{I u}$ & Ia & Im & CVEP & $\mathrm{TH}$ \\
\hline 1954-1988 & 228 & 16 & 1029 & 359 & 22,2 & 1,6 & 21 & 34,9 & $\mathrm{~B}_{1} \mathrm{rB}^{\prime} 4 \mathrm{a}^{\prime}$ \\
\hline $1955-1989$ & 240 & 13 & 1027 & 358 & 23,4 & 1,3 & 23 & 34,9 & B1 $r B^{\prime} 4 a^{\prime}$ \\
\hline $1956-1990$ & 237 & 11 & 1030 & 359 & 23,0 & 1,1 & 22 & 34,9 & B1 r r $4 \mathrm{a}^{\prime}$ \\
\hline $1957-1991$ & 255 & 13 & 1031 & 358 & 24,7 & 1,3 & 24 & 34,7 & $B 1 \times B^{\prime} 4 a^{\prime}$ \\
\hline $1958-1992$ & 257 & 15 & 1032 & 358 & 24,9 & 1,5 & 24 & 34,7 & B1 r $\mathrm{B}^{\prime} 4 \mathrm{a}^{\prime}$ \\
\hline 1959-1993 & 247 & 16 & 1036 & 359 & 23,8 & 1,5 & 23 & 34,7 & B1 r $\mathrm{B}^{\prime} 4 \mathrm{a}^{\circ}$ \\
\hline $1960-1994$ & 246 & 15 & 1038 & 360 & 23,7 & 1,4 & 23 & 34,7 & $\mathrm{~B} 1 \simeq \mathrm{B}^{\prime} 4 \mathrm{a}^{\prime}$ \\
\hline 1961-1995 & 249 & 14 & 1040 & 361 & 23,9 & 1,3 & 23 & 34,7 & $B 1 \times B^{\prime} 4 a^{+}$ \\
\hline 1962-1996 & 262 & 14 & 1040 & 362 & 25,2 & 1,3 & 24 & 34,8 & $\mathrm{Bl} \mathrm{rB}^{\prime} 4 \mathrm{a}^{\prime}$ \\
\hline
\end{tabular}

Legenda: $\mathrm{EXC}=$ Excedente hídrico; $\mathrm{DEF}=$ Deficiência hídrica; $\mathrm{EP}=$ Evapotranspiração potencial; $\mathrm{CV}=$ Concentração da Evapotranspiração potencial no veraão; $I u=$ Índice de umidade, Ia= Índice de aridez; Im= Índice efetivo de umidade ou Índice hídrico; CVEP= CV/Evapotranspiração potencial anual * 100 e $\mathrm{TH}=$ Classificação climática de Thornthwaite. 


\section{REFERÊNCIAS BIBLIOGRÁFICAS}

ASSIS, F. N. de Modelagem da ocorrência e da quantidade de chuva e de dias secos em Piracicaba - SP e Pelotas - RS. Piracicaba, 1996. 134p. Tese (Doutorado)- Escola Superior de Agricultura "Luiz de Queiroz", Universidade de São Paulo.

ASSIS, F. N. de; ARRUDA, H. V. de, PEREIRA, A R. Aplicações de estatística à climatologia: teoria e prática. Pelotas: UFPel, 1996.161p.

AYOADE, J. O. Introdução à climatologia para os trópicos. 4 ed. Rio de Janeiro: Editora Bertrand Brasil, 1996. 332p.

AZEVEDO, D.C. Chuvas no Brasil: regime, variabilidade e probabilidades de alturas mensais e anuais. Porto Alegre, 1974. 300p. Dissertação (M.S.) - Centro de Hidrologia Aplicada, Universidade Federal do Rio Grande do Sul.

BARBIERI, V.; TUON, R L.; ANGELOCCI, L. R. et al. Programa para microcomputador do balanço hidrico (Thornthwaite e Mather - 1955) para dados mensais e decendiais, normais e seqüênciais. In: CONGRESSO BRASILEIRO DE AGROMETEOROLOGIA, 7., Viçosa, 1991. Resumos. Viçosa: UFV, 1991. p.297299.

CAMARGO, A. P. Contribuição para a determinação da evapotranspiração potencial no Estado de São Paulo. Bragantia, n. 21, p. 163-213, 1962. 
CAMARGo, A. P. Balanço hídrico no Estado de São Paulo. Campinas: Instituto Agronômico de Campinas, (IAC. Boletim, 116). 1978. 28p.

CUNHA, G. R. Balanço hídrico climático. Agrometeorologia aplicada à irrigação.(coodernado por Homero Bergamaschi) Porto Alegre: UFRGS, n. 17, p. 63-84, 1992.

DEPARTAMENTO DE ÁGUAS E ENERGIA ELÉTRICA. Diagnóstico básico do plano de irrigação do Estado de São Paulo: atlas de desenvolvimento. São Paulo, 1973. 38p.

DE'CARLI, V. H. Adequação das épocas de cultivo na região de Manaus-AM, através do balanço hídrico seriado. Piracicaba, 1994. 73p Dissertação (Mestrado) - Escola Superior de Agricultura "Luiz de Queiroz", Universidade de São Paulo.

DOORENBOS, J.; KASSAN, A. H. Yield response to water. Rome:. FAO, 1979. 197p. (FAO. Irrigation and Drainage Paper, 33).

GALVANI, E. El Niño-oscilação sul (enos) e seus efeitos nas variações das chuvas na cidade de Piracicaba - SP. Piracicaba, 1996. 76p Dissertação (Mestrado) - Escola Superior de Agricultura "Luiz de Queiroz", Universidade de São Paulo.

GALVANI, E; PEREIRA, A R., VILLA NOVA, N. A. Tendência mensal e anual das chuvas em Piracicaba-SP. In: CONGRESSO BRASILEIRO DE AGROMETEOROLOGIA, 10., Piracicaba, 1997. Anais. Piracicaba: ESALQ, USP, 1997. p 277-279.

KÖPPEN, W. Climatologia: com un estudio de los climas de la tierra. Buenos Aires, 1931. 320p. 
MATA, G. J. Análise das disponibilidades hídricas das localidades de Barra de São Francisco, Boa Esperança e São Mateus, região norte do Estado do Espirito Santo. Piracicaba, 1991.113p. Tese (Doutorado)- Escola Superior de Agricultura "Luiz de Queiroz", Universidade de São Paulo.

MARTORANO L. G; PEREIRA, L. C; MARTINS CESAR, E. G, PEREIRA, I. C. B. Estudos climáticos do Estado do Pará, classificação climática (Köppen) e deficiência hídrica (Thornthwaite, Mather). Belém: SUDAM; EMBRAPA, SNLCS, 1993. 70p.

MARTORANO L. G.; MARIANO, Z. F.; BARBIERI, V.; SANTOS, R. M. N dos, SILVA, J. G et al. Caracterização climática de Piracicaba, SP: Análise das tendência de elementos meteorológicos. In: REUNIóN ARGENTINA $Y 1^{2}$ LATINOAMERICANA DE AGROMETEOROLOGÍA, $7^{\text {a }}$, Buenos Aires, 1997. Anais. Buenos Aires, 1997. p 31-32.

MARTORANO, L. G. Zoneamento agroecológico da quadrícula de Ribeirão Preto, SP, com base em características de solo, relevo e clima. Piracicaba, 1998. 77p Dissertação (Mestrado) - Escola Superior de Agricultura "Luiz de Queiroz", Universidade de São Paulo.

MONTEIRO, C. A F. A dinâmica climática e as chuvas no Estado de São Paulo estudo geográfico sob a forma de altas. São Paulo: USP, IGEOG, 1973. 129p.

MORAES, A. V. de C. Desenvolvimento e análise de modelos agrometeorológicos de estimativa de produtividade para a cultura da soja na região de Ribeirão Preto, SP. Piracicaba, 1998. 95p. Dissertação (Mestrado) - Escola Superior de Agricultura "Luiz de Queiroz", Universidade de São Paulo.

NATHAN, K. K. ; SINHA, S. K. Moisture deficit index evaluated for dry regions of Índia. Revista: Drought Network News, v. 8, n. 3, p. 14 -16, Oct. 1996. 
NIMER, E., BRANDÃO, A M. P. M. Balanço hídrico e clima da região dos cerrados. Rio de Janeiro: IBGE, 1989. 166p.

OMETTO, J. C. Bioclimatologia vegetal. São Paulo: Agronômica Ceres, 1981. 440p.

OMETTO, J. C. Registros e estimativas dos parâmetros meteorológicos da região de Piracicaba, SP. Piracicaba: FEALQ, 1991, 76p.

PELLEGRINO, G. Q. Análise espaço-temporal de dados hidrológicos da bacia do rio Piracicaba. Piracicaba, 1996. 117p. Dissertação (Mestrado.S.) - Escola Superior de Agricultura “Luiz de Queiroz”, Universidade de São Paulo.

PICCININI, M. R. D. Distribuições de probabilidade de precipitação de intensidade máxima para Piracicaba, SP. Piracicaba, 1996. 50p. Dissertação (Mestrado) - Escola Superior de Agricultura “Luiz de Queiroz”, Universidade de São Paulo.

PEREIRA, A. R, VIlla NOVA, N. A., SEDIYAMA, G. C. Evapo(transpi)ração. Piracicaba: FEALQ, 1997. 183p.

PINTO, H. S.; ZULLO NETO, J., ZULLO, S. A Oscilações pluviométricas temporais no estado de São Paulo. In: ENCONTRO DE ANÁLISE MULTIVARIADA E APLICAÇõES, Campinas, 1989. Resumos. Campinas: IMECC, UNICAMP, 1989, 31-39p.

SANSIGOLO, C. A. Características da estação chuvosa no Estado de São Paulo. . In: CONGRESSO BRASILEIRO DE AGROMETEOROLOGIA, 10., Piracicaba, 1997. Anais. Piracicaba: ESALQ, 1997. p 298-300. 
SANT'ANNA NETO, J, L. As chuvas no Estado de São Paulo: contribuição ao estudo da variabilidade e tendência da pluviosidade na perspectiva da análise geográfica. São Paulo, 1995. 201p. Tese (Doutorado) - Faculdade de Filosofia, Letras e Ciências Humanas - Universidade de São Paulo.

SANTOS, M.J.Z. dos. Variabilidade e tendência da chuva e sua relação com a produção agricola na região de Ribeirão Preto (SP). Rio Claro, 1992. 389p. Tese (LivreDocência) - Universidade Estadual Paulista, "Julio de Mesquita Filho".

SANTOS, P. M dos; FUNARI, F. L. Evapotranspiração potencial e balanço hídrico no Parque do Estado, São Paulo - SP. Revista Ciência e Cultura, v.28, n. 11, p 1325 $1329,1976$.

SANTOS, R. M.N. dos. Avaliação do índice de Palmer para a caracterização de períodos de seca no Estado de São Paulo. Piracicaba, 1998. 64p. Dissertação (Mestrado) Escola Superior de Agricultura "Luiz de Queiroz", Universidade de São Paulo.

SRIVASTAVA, A K. Effects of water stress on soybean productivity in Central India. Revista: Drought Network News, v. 8, n. 1, p. 7 - 8, Feb. 1996.

TARIFA, J. R. Sucessão de tipos de tempo e variação do balanço hídrico no extremo oeste paulista. São Paulo: USP, IGEOG, 1975. 20p. (Série Teses e Monografias, 8).

TOLEDO. G. S. de. Tipos de tempo e categorias climáticas na bacia do Alto Tietê (1968): ensaio metodológico. São Paulo, 1973. 200p. Tese (Doutorado) - Faculdade de Filosofia, Letras e Ciências Humanas - Universidade de São Paulo.

THORNTHWAITE, C. W. An approach toward a rational classification of climate. Geogr. Rev., n. 38, p. 55-94, 1948. 
THORNTHWAITE, C. W., MATHER, J. R. The water balance. Publications in Climatology. New Jersey: Drexel Institute of Technology, 1955. 104p.

TOMMASELLI, J. T. G. Deficiências hídricas nos solos das localidades de Cambará e Londrina, região Norte do Estado do Paraná. Piracicaba, 1992. 78p. Dissertação (Mestrado) - Escola Superior de Agricultura "Luiz de Queiroz", Universidade de São Paulo.

TUBELIS, A , NASCIMENTO, F. J. L. Meteorologia descritiva: fundamentos e aplicações brasileiras. São Paulo: Nobel, 1986.

VIANELLO, R. L. Meteorologia básica e aplicações. Viçosa: UFV, Impr. Univ., 1991. $460 \mathrm{p}$.

VIRMANI, S. M. Need, relevance, and objetives of the consultants' meeting on climatic classification. Índia: ICRISAT, 1980. p. xvii - xx. 